\title{
Direct Searches for Scalar Leptoquarks at the Run II Tevatron
}

\author{
A dissertation \\ submitted by \\ Daniel Edward Ryan \\ in partial fulfillment of the requirements \\ for the degree of \\ Doctor of Philosophy \\ in \\ Physics \\ TUFTS UNIVERSITY
}

August 2004

Advisor: Professor Krzysztof Sliwa 

This dissertation is dedicated to Destinie Boyd. Your love and endurance in the face of hardship has been a blessing. 


\title{
Direct Searches for Scalar Leptoquarks at the Run II Tevatron
}

\author{
by Daniel Edward Ryan
}

Adviser: Professor Krzysztof Sliwa

\begin{abstract}
This dissertation sets new limits on the mass of the scalar leptoquark from direct searches carried out at the Run II CDF detector using data from March 2001 to October 2003. The data analysed has a total time-integrated measured luminosity of $198 \mathrm{pb}^{-1}$ of $p \bar{p}$ collisions with $\sqrt{s}=1.96 \mathrm{TeV}$. Leptoquarks are assumed to be pair-produced and to decay into a lepton and a quark of the same generation. We consider two possible leptoquark decays: 1.) $\beta=B R(L Q \rightarrow \mu q)=1.0$, and 2.) $\beta=B R(L Q \rightarrow \mu q)=0.5$. For the $\beta=1$ channel, we focus on the signature represented by two isolated high- $p_{T}$ muons and two isolated high- $p_{T}$ jets. For the $\beta=1 / 2$ channel, we focus on the signature represented by one isolated high- $p_{T}$ muon, large missing transverse energy, and two isolated high- $p_{T}$ jets. No leptoquark signal is experimentally detected for either signature. Using the next to leading order theoretical cross section for scalar leptoquark production in $p \bar{p}$ collisions [1], we set new mass limits on second generation scalar leptoquarks. We exclude the existence of second generation scalar leptoquarks with masses below $221(175) \mathrm{GeV} / \mathrm{c}^{2}$ for the $\beta=1(1 / 2)$ channels.
\end{abstract}




\section{Acknowledgements}

I would like to thank my advisor Professor Krzysztof Sliwa for his support and guidance during my time as a graduate student. In matters of both physics and life he has been a source of wisdom and understanding. Secondly I am truly grateful for having the opportunity of working with Simona Rolli. I would like to thank her for giving me the opportunity to work on this analysis and having confidence in my abilities. She dealt with a countless number of my questions and guided me through the difficult processes of performing an analysis within a collaboration.

From Tufts I would like to thank the high-energy physics professors, including Professors Austin Napier, Bill Oliver, Tony Mann, and Jack Schneps. Thanks also go to Professors Roger Tobin, Peggy Cebe, Alexander Vilenkin and Allen Everett for their lectures and friendly conversation. I am grateful to Professor Yaacov Shapira for guiding me in my early years as a graduate student, and I would like to thank secretaries Waltye Greene, Jackie DiMichele, and Jean Intoppa for taking care of so many administrative issues. Special thanks go to Jim Cyr for taking care of technical issues in physics labs, his friendliness and lending me his electronics equipment. For my colleagues and friends I am especially grateful to Ben Whitehouse for answering so many of my C ++ questions, Hao Sun for helping with cross checks of the analysis code, and Liz Heider and Matt Hare for their fellowship.

From CDF I would like to thank Marcin Wolter for showing me the ropes of the low voltage power supply monitoring system and for the smoked cheese and Starka. Thanks go to Steve Hahn, Dee Hahn, J.C. Yun, and Roberto Davila for making the control room a happy place and recognizing my efforts on the low voltage power supply monitoring. Many thanks go to Müge Karagöz Ünel for helping me understand countless aspects of this analysis, including evtNtuples, the muon detectors, the event display, and the muon

efficiencies. I would like to thank the Exotics group for their suggestions and insights to this analysis during its blessing. I would like especially to thank Beate Heinemann and Stephan Lammel for answering my questions and asking me to represent the Tevatron at the 2004 DIS conference.

From DØ I would like to thank Professor Pushpa Bhat for inviting me to work with her 
and the Run II Advanced Analysis Group on Independent Component Analysis and other multivariate analysis methods. I am especially grateful to my friend and colleague Kevin Black. Even from our days as undergraduates his example has helped me stay motivated and interested in physics.

I would like to give big thanks my family. I thank my sister for lending her ear during difficult times and my brother for giving me so much moral support. I will be forever thankful to my parents, who have given me so much love and encouragement. Without them this dissertation would not have been possible. Finally, I would like to thank my Fiancée. Her patience, love and willingness have helped me to move ahead. 


\section{Contents}

I Theoretical Motivation $\quad 2$

1 The Standard Model 3

1.1 Particles and Their Interactions f . . . . . . . . . . . . . . 3

1.1.1 Quarks and Leptons . . . . . . . . . . . . . . . . . . . . 4

1.1.2 Gauge Bosons . . . . . . . . . . . . . . . . . . 5

1.2 Theory of Gauge Fields . . . . . . . . . . . . . . . 7

1.3 Symmetries of the Standard Model . . . . . . . . . . . . . . . . . 9

1.4 The Hierarchy Problem . . . . . . . . . . . . . . . . . . . 11

1.5 Beyond the Standard Model f . . . . . . . . . . . . . . . . 14

2 Leptoquarks $\quad \mathbf{1 6}$

2.1 Leptoquark Theories . . . . . . . . . . . . . . . . . . . 16

2.1.1 Grand Unified Theories . . . . . . . . . . . . . . 17

2.1.2 Dynamic Symmetry Breaking and Composite Models . . . . . . . . . 19

2.1 .3 SUSY . . . . . . . . . . . . . . . . . . 20

2.2 The Buchmüller-Rückle-Wyler Leptoquark Classification . . . . . . . . . . . . 21

2.3 Leptoquark Production . . . . . . . . . . . . . . . . . . . . 23

2.4 Leading Order Cross Section _ . . . . . . . . . . . . . . . 26

2.5 Next-to-Leading Order Cross Section . . . . . . . . . . . . 27

2.5.1 Virtual Corrections . . . . . . . . . . . . . . . 27

2.5.2 Bremsstrahlung of Gluons . . . . . . . . . . . . . . 28

2.5.3 Compton Process . . . . . . . . . . . . . . . . . . . . . 29

2.5.4 Numerical Calculation . . . . . . . . . . . . . . . . . . . . . . 29

2.6 Leptoquark Decay . . . . . . . . . . . . . . . . . . 31

2.7 Existing Leptoquark Mass Limits _. . . . . . . . . . . . . . . . 33 
$\begin{array}{lll}\text { II } & \text { Experimental Apparatus } & 37\end{array}$

3 The Apparatus $\quad 38$

3.1 Run II Proton Producton and Acceleration . . . . . . . . . . . . . . . 38

3.1.1 Proton Production and Acceleration . . . . . . . . . . . . . . 39

3.1.2 Antiproton Production and Acceleration . . . . . . . . . . . . . 41

3.2 The Run II Collider Detector at Fermilab . . . . . . . . . . . . . . . . . 43

3.2 .1 Tracking . . . . . . . . . . . . . . . . . 45

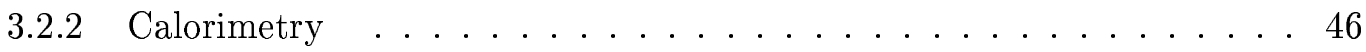

3.2.3 Muon Detectors . . . . . . . . . . . . . . . . 52

3.2 .4 Luminosity Detectors . . . . . . . . . . . . . . . . 59

3.3 Triggering and Data Acquisition . . . . . . . . . . . . . 60

$\begin{array}{llr}\text { III Analysis } & 65\end{array}$

4 Data Sample $\quad 66$

4.1 Analysis Overview and Strategy . . . . . . . . . . . . . . . 66

4.2 The Inclusive High- $p_{T}$ Data Sample . . . . . . . . . . . . . . . . . 68

4.3 Leptoquark Signal Monte Carlo _. . . . . . . . . . . . . . . . . 69

4.4 Background Monte Carlo . . . . . . . . . . . . . . . . . . 70

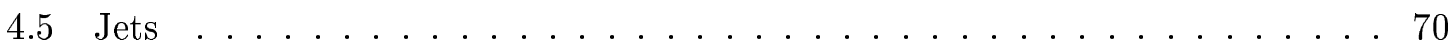

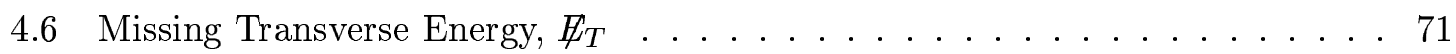

4.7 Muons . . . . . . . . . . . . . . . . . . . . 71

4.8 Preselection Efficiencies . . . . . . . . . . . . . . . . 72

4.8.1 Muon Identification and Isolation Efficiency _ . . . . . . . . . . 73

4.8.2 Muon Trigger Efficiency . . . . . . . . . . . . . . . . . . . . 75

4.8.3 Muon Reconstruction Efficiency . . . . . . . . . . . . . 76

4.8.4 Z-Vertex Efficiency . . . . . . . . . . . . . . . 76

4.8 .5 Cosmic Ray Rejection . . . . . . . . . . . . . . . . 77

5 Search for Scalar Leptoquarks in the $\mu q \mu q$ Channel $\quad 78$

5.1 Event-type Efficiency Calculation . . . . . . . . . . . . 78

5.2 Acceptance and Kinematical Efficiency Calculation . . . . . . . . . . . . . . 79

5.3 Background calculation in the $\mu q \mu q$ channel $\ldots \ldots \ldots$. . . . . . 85

5.3 .1 DY +2 jets .................... 85

5.3 .2 Top quark production: $t \bar{t} \ldots \ldots \ldots \ldots$. . . . . . . . 86

5.3 .3 QCD $/$ Fakes . . . . . . . . . . . . . 86

5.4 Cross-check with the $Z$-boson cross section $\ldots \ldots \ldots$. . . . . 87 
5.5 Systematic Uncertainty . . . . . . . . . . . . . . . . . . . . . . . . . . 87

5.6 Cross Section Upper Limit Calculation _ . . . . . . . . . . . 88

6 Search for Scalar Leptoquarks in the $\mu q \nu q$ Channel 94

6.1 Event-type efficiency calculation . . . . . . . . . . . . . . . . 94

6.2 Acceptance and kinematical efficiency calculation . . . . . . . . . . . 95

6.2 .1 Mass Cut . . . . . . . . . . . . . . . 96

6.2 .2 Total Acceptances . . . . . . . . . . . . . . . . . 97

6.3 Background calculation in the $\mu q \nu q$ channel $\ldots \ldots . \ldots . \ldots . \ldots 9$

6.4 Cross-check with the $W+\geq 2$ jets process . . . . . . . . . . . . . . 101

6.5 Systematic uncertainty . . . . . . . . . . . . . . . . . 102

6.6 Cross section limit . . . . . . . . . . . . . . . . . . . 102

$\begin{array}{lll}7 & \text { Epilogue } & 111\end{array}$

$\begin{array}{ll}\text { A Muon ID and Isolation Efficiencies } & 115\end{array}$

B ID and Isolation Dependence 118

B.1 The Higgs Mechanism . . . . . . . . . . . . . . . . . . . . . . . . . . . . . 119 


\section{Direct Searches for Scalar Leptoquarks at the Run II Tevatron}




\section{Part I}

\section{Theoretical Motivation}




\section{Chapter 1}

\section{The Standard Model}

In this chapter the Standard Model of particles and their interactions is discussed. The theory of gauge fields is introduced so that the discussion of U(1), SU(2) and SU(3) invariance is connected to forces and symmetries. The chapter concludes with inelegancies and incompletenesses of the Standard Model as motivation for study of extensions to the Standard Model.

\subsection{Particles and Their Interactions}

Particle physics attempts to understand the fundamental constituents of matter and their interactions. The fermions are the smallest known constituents of matter. They are spin-1/2 particles comprising two families: the leptons and quarks. The lepton and quark families are each divided into three generations of lepton and quark doublets. The masses of the particles in each generation are heavier than the generation preceding it. The interaction forces of the leptons and quarks are mediated by the exchange of fundamental gauge bosons.

There are four known forces governing the interactions of matter at low energies: strong, electromagnetic, weak, and gravitational. Properties of these forces are shown in Table 1.1. The strong force is attractive and has a very short range of interaction. The strong forces are felt by quarks and are mediated by the exchange of gluons. Protons and neutrons, which comprise the nucleii of atoms, are comprised of quarks which are bound together by the strong forces. The strong force is also responsible for the binding of protons and neutrons in the atomic nucleus. The potential of the strong force is proportional to the square of the distance between particles much like the forces associated with springs. The electromagnetic force is proportional to the inverse of the square of the particle distance. This force affects all particles having electric charge and is mediated by the exchange of photons. The electromagnetic force is attractive if the particles have opposite charge and 


\begin{tabular}{lllll}
\hline Interaction & Mediator & $\begin{array}{l}\text { Coupling Constant } \\
\left(M c^{2}=\mathrm{GeV}\right)\end{array}$ & Range $(\mathrm{m})$ & Typical Lifetime (s) \\
\hline \hline strong & $\mathrm{g}$ & $<1$ & $\leq 10^{-15}$ & $10^{-23}$ \\
electromagnetic & $\gamma$ & $1 / 137$ & $\infty$ & $10^{-20}$ \\
weak & $W^{ \pm}, Z$ & $10^{-6}$ & $10^{-18}$ & $10^{-10}$ \\
gravity & graviton & $10^{-40}$ & $\infty$ & \\
\hline
\end{tabular}

Table 1.1: The fundamental interactions and their properties. The typical interaction lifetime denotes the timescale for the decay of an unstable particle via that fundamental interaction. For example the mean lifetime for decay through a weak interaction is $10^{-10} \mathrm{~s}$.

repulsive if they have the same charge. The strength of electromagnetic interactions is approximately two orders of magnitude smaller than the strong force. The weak force is approximately six orders of magnitude smaller than the strong force. The weak force also has a short range of interaction and is mediated by heavy gauge bosons. This force affects quarks, leptons and the gauge bosons excluding gluons and is responsible for neutron decay. Finally, the gravitational force is a purely attractive force between two particles proportional to the product of their masses and inversely proportional to the square of their spatial separation. Gravity is felt by particles of any type and is roughly 40 orders of magnitude weaker than the strong force. At presently accessible energy scales its effect only becomes large when the interacting bodies have a large accumulation of mass as is the case in interplanetary systems.

\subsubsection{Quarks and Leptons}

Quarks are particles having fractional electric charge and intrinsic spin $1 / 2$. There are six kinds of quarks, denoted by their intrinsic "flavor": up $(u)$, down $(d)$, strange $(s)$, charmed $(c)$, bottom $(b)$, top $(t)$. The quarks are listed in order of increasing mass. The last four quarks are unstable particles and decay into lighter, more stable particles. The $u$ and $d$ quarks are stable and comprise particles existing as their bound states. There are two categories of bound state quarks: mesons and baryons. A meson is a bound state of a quark and antiquark, e.g. a $\pi^{+}$is a meson having the quark content $u \bar{d}$. A baryon is a bound state of 3 quarks. A proton is an example of a baryon and is the bound state of two $u$ quarks and one $d$ quark. Baryons are fermions due to their odd number of quarks, and mesons are bosons due to their even number. Both baryons and mesons have integer charge, while quarks have fractional charge. Baryons and mesons comprise the group of particles called hadrons. Quarks are categorized into three generations. Table 1.2 shows the three quark generations. 


\begin{tabular}{|rcccc|}
\hline Generation: & I & II & III & $Q /|e|$ \\
\hline \hline Quarks & $u$ & $c$ & $t$ & $+2 / 3$ \\
& $d$ & $s$ & $b$ & $-1 / 3$ \\
\hline Leptons & $e$ & $\mu$ & $\tau$ & -1 \\
& $\nu_{e}$ & $\nu_{\mu}$ & $\nu_{\tau}$ & 0 \\
\hline
\end{tabular}

Table 1.2: The quark and lepton generations and their respective charges. $Q /|e|$ denotes the charge relative to the charge of the electron. The corresponding antiparticles have the opposite charge.

Because quarks are fermions, many of their bound states appear to be in direct contradiction to Fermi statistics. To avoid this contradiction quarks are also assumed have an intrinsic property called color. There are 3 types of color which are denoted by $R$ (red), $B$ (blue), or $G$ (green). Likewise, antiquarks have color $\bar{R}$ (anti-red), $\bar{B}$ (anti-blue), or $\bar{G}$ (antigreen). The exchange of color charge in strong interactions is analogous to the exchange of electric charge in electromagnetic interactions. Quarks are postulated to come in three colors due to experimental constraints from cross section ratios and pion decay rates (see for example [85]). In addition to the restriction of unit charge, bound quark states must be "colorless". The quark colors are defined such that any $R G B, \bar{R} \bar{G} \bar{B}$, or color-anticolor combination is colorless. This restriction accommodates the experimental constraint that singly existing quarks and many otherwise possible bound quark states have not been observed.

Leptons and their antiparticles are spin- $1 / 2$ particles having an electric charge of $\pm e$ or 0 , where $e$ is the charge of the electron. The charged leptons are the $e$ (electron), $\mu$ (muon) and $\tau$ (tau), and the neutral leptons are the $\nu_{e}$ (electron neutrino), $\nu_{\mu}$ (muon neutrino) and $\nu_{\tau}$ (tau neutrino). The charged leptons interact via the electromagnetic and weak forces whereas the neutrinos interact via only the weak force. There is no experimental evidence of right-handed neutrinos. Analogous to the generations of quarks, the second and thirdgeneration charged leptons are not stable and will decay via the weak forces to lighter stable particles. The electron is the lightest lepton having a mass of $M_{e}=0.511 \mathrm{MeV} / \mathrm{c}^{2}$. The next heaviest charged lepton is the muon weighing about three orders of magnitude larger than the electron and having a mean lifetime of about $2 \mu \mathrm{s}$. The mass of the tau lepton is the heaviest lepton having a mass of $M_{\tau}=1.78 \mathrm{GeV} / \mathrm{c}^{2}$. For simplicity neutrinos were assumed to be massless. However, recent measurements of neutrino oscillations [57] indicate that neutrinos may have very small masses.

\subsubsection{Gauge Bosons}

Gauge bosons are integer spin particles which mediate particle forces through their virtual exchange between the constituents of matter. Therefore each gauge boson corresponds 
to a fundamental particle interaction: the strong force is mediated by gluons; the weak forces is mediated by the charged $W$ 's and the neutral $Z$-boson; and the electromagnetic force is mediated by massless neutral photons. It is speculated that gravity is mediated by massless gravitons having intrinsic spin-2, although its strength at current experiments is too weak at the particle level to measure.

The equivalence of particle forces and the exchange of virtual bosons is most easily understood in quantum electrodynamics (QED). Charged particles emit photons which are absorbed in the presence of another charged particle. To conserve energy the emitting particle must recoil with the corresponding energy of the photon. The absorbing particle also recoils proportional to the energy of the photon and the net momentum and energy is conserved. The overall observed effect is that of electromagnetic attraction or repulsion. All particles having the property of non-zero electric charge are influenced by electromagnetic forces and therefore interact via virtual or real photon exchange. Because photons are themselves electrically neutral, they do not interact with other photons.

Quarks interact via the strong force by virtue of their intrinsic property of color. In order to be carriers of the strong force, gluons must themselves carry color and therefore are capable of acting on other gluons. The attractive strong force is unique in that its force is directly proportional to the square of the spatial separation. This means that the strong force actually increases as the strongly bound quarks move further apart. This is in contrast to the weak and electromagnetic interactions which decrease with distance. As two quarks pull away from one another, the confining energy ( $\left.\mathrm{k} \quad 1 \mathrm{GeV} \mathrm{fm}^{-1}\right)$ actually becomes large enough that it is more energetically favorable to create a quark-antiquark pair such that each of the new quarks pairs itself with one of the original quarks. Thus the spatial distance between the original quark-pair is reduced in half and two distinct bound quark states can emerge from one bound state. This process is known as hadronization.

The weak force is another short range interaction. The short range of interaction is accounted for by the heavy mass of the weak force carriers. The fermions and massive gauge bosons undergo weak interactions. The exchange of charged $W$-bosons is observed through charged currents. For example in $\beta$-decay $n \rightarrow p+e^{-}+\bar{\nu}_{e}$ as shown in Figure 1.1, a $W^{-}$ is emitted from a $d$-quark in the neutron and decays into an electron-neutrino pair. The $d$-quark gives up its charge and becomes a $u$-quark thus converting the neutrally charged neutron to a positively charged proton. So far it has been observed that the $Z$-boson is only exchanged among fermions within a generation. Experiments have placed very strict limits on the exclusion of flavor changing neutral currents (FCNC).

For reasons unknown, the weak force does not interact with right-handed particles. A left(right)-handed particle has the property that its spin is antiparallel(parallel) to its direction of motion. To accommodate this phenomenological constraint, leptons and quarks are 


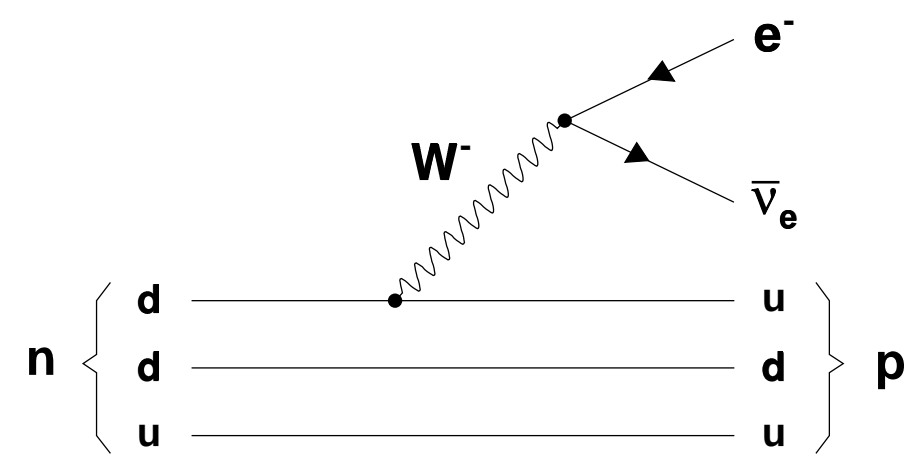

Figure 1.1: Feynman diagram of nuclear $\beta$-decay.

categorized into left-handed doublets and right-handed singlets. However, to explain certain observations such as strangeness-changing weak interactions, the left-handed quark doublets are not arranged in their mass eigenstates. Rather they are expressed as a linear combination of their physical quark states through a non-diagonal $3 \times 3$ mixing matrix called the CKM matrix. The degrees of freedom associated with this mixing matrix can be reduced to three angles and one phase angle for a total of four experimentally determined parameters. Because this phase angle is not invariant under time reversal, the CKM matrix allows for the possibility of decays which do not respect combined transformations of charge and parity (CP transformations). This deduction follows from the CPT theorem which states that all interactions must respect the combined transformations of charge conjugation, parity, and time reversal. The conservation of CPT transformations is consistent with all observations to date. $\mathrm{CP}$ violation has been observed in the decay of neutral kaons and $b$-mesons.

\subsection{Theory of Gauge Fields}

This section provides a mathematical formulation which is employed by the Standard Model to account for the phenomenology of the particles and their interactions described in the last section. In gauge field theory particles are described as quantized fields appearing in the Lagrangian density, $\mathscr{L}$. The motivation for using the theory of gauge fields to describe particle interactions lies in its ability to connect conserved quantities with symmetries. This connection is proven in Noether's Theorem which states that for each continuous symmetry in a system there corresponds a conserved quantity (for discussion see [66]). For example, linear transformations in space correspond to the conservation of momentum along the direction of translation. In the theory of gauge fields, any continuous field transformation 
which leaves unchanged the action of the Lagrangian, $\mathcal{S}=\int d x \mathscr{L}(\varphi, \partial \varphi)$, corresponds to a conserved quantity. The Standard Model framework is based on local gauge field invariances. The symmetries corresponding to these invariances dictate the interactions of particles and allow for a more simplified particle, and particle interaction, classification scheme.

The QED Lagrangian can be derived by requiring local gauge invariance of the Dirac Lagrangian for a free particle having mass $m$ :

$$
\mathscr{L}_{\text {Dirac }}=i \bar{\varphi} \gamma^{\mu} \partial_{\mu} \varphi-m \bar{\varphi} \varphi
$$

Equation (1.1) is manifestly globally invariant under phase transformations. In group theory phase transitions are described by the $U(1)$ gauge group transformations which are the set of all one-dimensional unitary matrices: $U(\theta)=e^{i q \theta}$. For the transformation $\varphi \rightarrow$ $e^{i q \theta} \varphi$, the Lagrangian (1.1) remains unchanged, and the charge, $q$, is a conserved quantity. However, this is not the case when requiring (1.1) to be invariant under a local $U(1)$ gauge transformation which, unlike a global gauge transformation, is space-time dependent: $\varphi \rightarrow$ $e^{i q \theta(x)} \varphi$.

Equation (1.1) can be made locally gauge invariant by introducing an extra term in the Lagrangian which will cancel the factor resulting from the derivative of the space-time dependent $\theta(x)$. This extra term is folded into the derivative to make a "covariant derivative":

$$
\mathcal{D}_{\mu} \equiv \partial_{\mu}+i q A_{\mu}
$$

where $A_{\mu}$ is a vector gauge field and as a rule transforms under gauge transformations as $A_{\mu} \rightarrow A_{\mu}+\frac{1}{q} \partial_{\mu} \theta$. The gauge field $A_{\mu}$ can be fixed in this way since the derivatives of fields will not affect the Euler-Lagrangian equations. The result of using this covariant derivative in $(1.1)$ is

$$
\mathscr{L}^{\prime}=i \varphi \gamma^{\mu} \partial_{\mu} \varphi-q \varphi \gamma^{\mu} \varphi A_{\mu}-m \bar{\varphi} \varphi
$$

Equation (1.1) is promoted to a locally invariant Lagrangian by replacing the derivative with a covariant one. However, since the covariant derivative introduces a new vector gauge field, $A_{\mu}$, the full Lagrangian must have a corresponding free Lagrangian term for this field. Since $A_{\mu}$ is a vector field, it can be described by the Lagrangian for a vector field (see [63]), i.e.

$$
\mathscr{L}_{\text {Free }}=-\frac{1}{4} F^{\mu \nu} F_{\mu \nu}+\frac{1}{2} m_{A}^{2} A^{\nu} A_{\nu}
$$

Since the second term in (1.4) is not locally gauge invariant, it is necessary to require that the mass of the vector gauge field be zero, i.e. $m_{A}=0$. The change to a covariant derivative 
and the addition of a free term for the resulting vector field results in a new Lagrangian:

$$
\mathscr{L}_{Q E D}=\bar{\varphi}\left(i \gamma^{\mu} \partial_{\mu}-m\right) \varphi-\frac{1}{4} F^{\mu \nu} F_{\mu \nu}-q \bar{\varphi} \gamma^{\mu} \varphi A_{\mu}
$$

Equation (1.5) is well known as the Lagrangian for an electromagnetic field, where $A_{\mu}$ is the electromagnetic potential, and $q$ is the electric charge which couples the electromagnetic field (photons) to the spinor fields (electrons and positrons) by a factor of $q$.

By requiring that the Dirac Lagrangian for a free spinor field be locally invariant under phase transformations, the Lagrangian for spinor fields interacting with the electromagnetic potential is obtained. The last two terms in (1.5) appear in the Maxwell Lagrangian, where the charge density is given by $J^{\mu}=q\left(\bar{\varphi} \gamma^{\mu} \varphi\right)$. Though it is sufficient to only require global phase invariance to explain conservation of charge, it takes local phase invariance of (1.1) to reproduce the Maxwell Lagrangian (last 2 terms of (1.5)) for charged currents.

\subsection{Symmetries of the Standard Model}

The symmetry group describing the interactions in the Standard Model is:

$$
S U(3)_{c} \times S U(2)_{L} \times U(1)_{L}
$$

In analogy to QED, the strong interactions are described in Quantum Chromodynamics using the group representation $S U(3)_{c}$. The "c" denotes that these interactions are coupled to the gauge fields. The requirement of local gauge transformation of the $S U(3)$ group results in vector fields, or gluons, which couple to the fermions by $\alpha_{s}$. Unlike the $U(1)$ group, the $S U(3)$ group is non-Abelian which means that the generators of the group do not commute. Mathematically this results in gluon-gluon coupling which indicates that gluons must themselves have color charge. There are three types of color charges. All gluons carry one color and one anticolor charge resulting in 9 possible gluon states which are transformed by the generators of the $S U(3)_{c}$ group. One gluon state is a colorless singlet and therefore there are only 8 active carriers of the color charge. The coupling between the vector gauge fields of a locally invariant gauge group is indicative of non-Abelian theories.

Though the electromagnetic forces are well described by the $U(1)$ group, it was shown by Weinberg, Salam and Glashow that the weak and electromagnetic forces were manifestations of an underlying "electroweak" force represented by the $S U(2)_{L} \times U(1)_{Y}$ group. These group transformations are associated with particles having weak isospin $(I)$ with coupling $g$ and weak hypercharge $(Y)$ with $g^{\prime}$ respectively. The couplings are parameterized in terms of the Weinberg angle given by $\cos \theta_{W}=g / \sqrt{g^{2}+g^{\prime 2}}=g / \bar{g}$. The weak hypercharge behaves in the same way under gauge transformations as the EM field in Section 1.2. Like $S U(3)$, the 
$S U(2)$ group is non-Abelian which means the weak gauge fields can couple to themselves. In the $U(1)$ gauge group, one massless vector field is needed to promote the group to local gauge invariance. In the case of the $S U(2)$ group, the gauge sector is required to have 3 massless vector gauge fields, $\left(B^{-}, B^{0}, B^{+}\right)$. These gauge bosons are carriers of the weak isospin.

The short range of weak interactions, $\mathcal{O}\left(\sim 10^{-18} \mathrm{~m}\right)$, indicates that the particles mediating the weak force must be very massive. However, the gauge bosons associated with the $S U(2)_{L}$ group, $B^{ \pm}, B^{0}$, are massless. This disparity was accounted for by Weinberg, Salam and Glashow when they proposed the electroweak theory (for discussion see [85]). The weak gauge bosons are endowed with mass while keeping the Lagrangian locally gauge invariant through a process called the Higgs Mechanism. A detailed discussion of the method is in Appendix B.1. In brief, the Higgs field is a scalar field which has a vacuum potential with degenerate ground states. The symmetry of the Higgs Lagrangian is broken when a particular value for the ground state, $\nu$, is chosen which results in a massless scalar gauge field known as a "Goldstone Boson." This massless Goldstone boson Lagrangian term is absorbed by the gauge bosons through requiring local phase invariance of the Higgs field. By fixing the gauge of the scalar Higgs field, a degree of freedom is afforded to the weak vector gauge fields which appears as a mass term.

The masses of the $Z$-boson and $W^{ \pm}$-bosons are related to the electroweak coupling and vacuum ground state by:

$$
m_{Z}=\frac{1}{2} \bar{g} \nu, m_{W}=\frac{1}{2} g \nu,
$$

where the ratio of the $W$ and $Z$ masses is proportional to the Weinberg angle $m_{W} / m_{Z}=$ $\cos \theta_{W}$. The 2002 Particle Data Group lists the value $\sin ^{2} \theta_{W}=0.2113(15)$. Using the relation $G_{F} / \sqrt{2}=g^{2} / 8 m_{W}^{2}$, where $G_{F}=1.16637(1) \times 10^{-5} \mathrm{GeV} / \mathrm{c}^{2}$ is the Fermi constant [88], we can use (1.7) to obtain an estimate for the vacuum expectation value used to break the $\mathrm{SU}(2) \times \mathrm{U}(1)$ symmetry:

$$
\nu=\left(\frac{1}{\sqrt{2} G_{F}}\right)^{1 / 2} \sim 246 G e V .
$$

Equation (1.8) represents the energy above which electroweak symmetry is restored.

The Higgs mechanism is also employed in another sector of the Standard Model Lagrangian. The method of requiring local $S U(2)$ gauge invariance for the final Standard Model Lagrangian is problematic for spin- $1 / 2$ particles. The left and right-handed helicity states of the fermions transform differently under rotations of the $S U(2)$ type, which makes it impossible to construct a Lagrangian which is locally $S U(2) \times U(1)$ invariant. The 
local gauge invariance of the Lagrangian could be restored by replacement of the partial derivatives with covariant ones having a particular helicity, i.e.:

$$
\begin{aligned}
D_{\mu} \psi_{R} & =\left(\partial_{\mu}+i g^{\prime} B_{\mu}\right) \psi_{R} \\
D_{\mu} \psi_{L} & =\left(\partial_{\mu}+\frac{i}{2} g^{\prime} B_{\mu}-\frac{i}{2} g \sigma_{i} W_{\mu}^{i}\right) \psi_{L} .
\end{aligned}
$$

However the mass term is a scalar operator which mixes the left/right-helicity states. This spoils the $S U(2)$ invariance of the Lagrangian unless the mass terms vanish. The Standard Model treats this problem by endowing masses to the fermions using the same method that was used to endow masses to the gauge bosons. The fermion interactions can be given by [26]:

$$
\begin{aligned}
i \gamma^{\mu} D_{\mu} \psi_{R}-\lambda_{f} \phi^{\dagger} \psi_{L} & =0 \\
i \gamma^{\mu} D_{\mu} \psi_{L}-\lambda_{f} \phi^{\dagger} \psi_{R} & =0 .
\end{aligned}
$$

Once the field shifts such that $\langle\phi\rangle_{0} \neq 0$, the fermion will absorb the resulting Goldstone boson and acquire mass.

Though this method will keep the Lagrangian locally $S U(2)$ invariant, it comes at the price of inelegance. There is nothing to fix the coupling constants, $\lambda_{f}$, in (1.10). Therefore, for each different massive fermion there must correspond a different coupling constant, i.e. $\lambda_{f_{e}}$ for elections, $\lambda_{f_{\nu_{e}}}$ for electron-neutrinos, etc. Each must be tuned to achieve the experimentally measured fermion mass. This is a good indication that the Standard Model is an incomplete theory. In all, the Higgs mechanism endows masses for 15 discovered particles in the Standard Model. However, a physical Higgs particle has yet to be experimentally detected.

\subsection{The Hierarchy Problem}

The Hierarchy Problem, sometimes called the "naturalness problem" is the most notable inelegance of the Standard Model and appears when considering the radiative corrections to the Higgs mass. For an electron the Feynman diagram for the 2-point function at vanishing external momentum computed at the one-loop level is:

The energy from this contribution as prescribed by Feynman diagram rules is:

$$
\begin{aligned}
\Sigma_{F}(p) & =\int \frac{d^{4} k}{(2 \pi)^{4}}\left(-i e \gamma^{\mu}\right) \frac{i}{\not k-m_{e}}\left(-i e \gamma^{\nu}\right) \frac{-i g_{\mu \nu}}{k^{2}} \\
& =-e^{2} \int \frac{d^{4} k}{(2 \pi)^{4}} \frac{1}{k^{2}\left(k^{2}-m_{e}^{2}\right)} \gamma^{\mu}\left(\not k-m_{e}\right) \gamma_{\mu},
\end{aligned}
$$




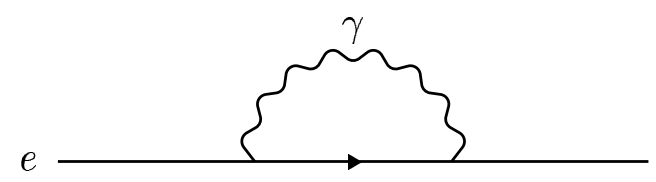

Figure 1.2: The electron self-energy diagram in QED.

where the "slash" notation denotes $\not k \equiv \gamma^{\mu} k$. The four-dimensional volume element can be written $d^{4} k=k^{3} d k d \omega$. By counting powers of $k$ in the numerator and denominator of Equation 1.11, one can deduce that it is divergent. After some algebraic manipulation and integrating over all angles, the most divergent part of (1.11) is:

$$
\int_{0}^{\infty} \frac{1}{k^{4}} k^{3} d k=\left.\ln k\right|^{\infty}=\ln (\infty)
$$

The fact that (1.11) diverges can be seen as a consequence of its range of $k$ integration. Physically interpreted, there become an infinite number of loop corrections at high-momentums, or equivalently very small space-times.

The solution to this problem of small space-time divergences is the method of renormalization. There are many renormalization schemes, but all of them share the same premise. The parameters of the standard model, such as coupling constants and masses, are in fact themselves divergent. However, what experimentalists physically measure are not the bare parameters which are presumably unmeasurable. Rather what is measured are effective parameters containing extra factors, e.g. $m=m_{0}+\delta m$ and $g=g_{0}+\delta g$. Loosely speaking, renormalization is a prescription for arranging the divergences in the bare masses and couplings to exactly cancel the high-momentum infinities of a theory thus making the theory finite to all orders of perturbation. However, since there are only a finite number of parameters in a theory, not all theories are renormalizable.

If the bare parameters are to absorb the divergences of a theory, these corrections should correspond in some way to the severity of the divergence. As an example, it can be shown that to first order the one-loop correction to the electron in QED theory (Figure 1.2) will give [66]:

$$
m_{e}=m_{e, 0}\left[1+\frac{3}{2} \frac{\alpha}{\pi} \ln \frac{\Lambda_{e}}{m_{e, 0}}+\cdots\right]
$$

where $\Lambda_{e}$ is the upper limit of integration in (1.11). Taking $\Lambda_{e}$ to be the entire mass of the universe $\left(10^{79} \mathrm{GeV} / \mathrm{c}^{2}\right)$ shifts the electron mass by $m_{e} \simeq 1.7 m_{e, 0}$. This indicates that at least with logarithmic divergences, the normalization process of absorbing divergences into 
renormalized parameters is reasonable.

However, in the case of first-order corrections to the SM Higgs particle from internal fermion loops the divergence becomes worse. Figure 1.3 shows a diagram of the 2-point function from this one-loop correction. The 2-point energy correction from this fermion

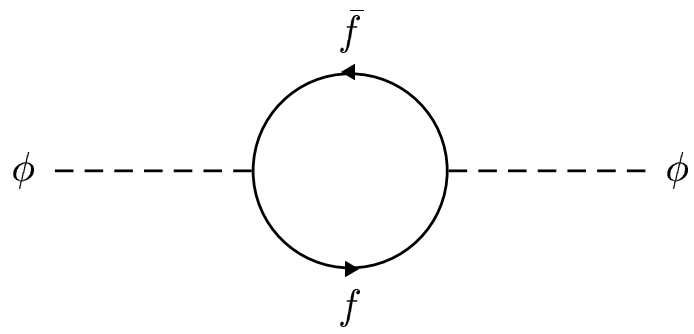

Figure 1.3: The fermion one-loop contribution to the SM Higgs self-energy diagram.

loop with coupling $\lambda_{f}$ is:

$$
\begin{aligned}
\left(\delta m_{h}\right)_{a}^{2} & =-\int \frac{d^{4} k}{(2 \pi)^{4}} \operatorname{Tr}\left[\left(\frac{i \lambda_{f}}{\sqrt{2}}\right) \frac{i}{\not k-m_{f}}\left(\frac{i \lambda_{f}}{\sqrt{2}}\right) \frac{i}{\not k-m_{f}}\right] \\
& =-2 \lambda_{f}^{2} \int \frac{d^{4} k}{(2 \pi)^{4}} \frac{k^{2}+m_{f}^{2}}{\left(k^{2}-m_{f}^{2}\right)^{2}} \\
& =-2 \lambda_{f}^{2} \int \frac{d^{4} k}{(2 \pi)^{4}}\left(\frac{1}{k^{2}-m_{f}^{2}}+\frac{2 m_{f}^{2}}{\left(k^{2}-m_{f}^{2}\right)^{2}}\right) .
\end{aligned}
$$

Counting powers of $k$ in the first term of (1.13) gives: $\int^{\infty} k^{3} \frac{1}{k^{2}} d k=\left.k^{2}\right|^{\infty}=(\infty)^{2}$. This time the divergence of the 2-point energy correction is quadratic. Calculating (1.13) results in a mass correction of [66]:

$$
\begin{aligned}
\left(\delta m_{h}\right)_{a}^{2}= & -\frac{\lambda_{f}^{2}}{8 \pi^{2}}\left[\Lambda^{2}+\left(m_{h, 0}-6 m_{f}^{2}\right) \log \left(\frac{\Lambda}{m_{f}}\right)\right. \\
& \left.+\frac{1}{2}\left(4 m_{f}^{2}-m_{h, 0}^{2}\right)\left(1+I_{1}\left(\frac{m_{h, 0}^{2}}{m_{f}^{2}}\right)\right)\right]+\mathcal{O}\left(\frac{1}{\Lambda^{2}}\right),
\end{aligned}
$$

where $I_{1}(a) \equiv \int_{0}^{1} d x \log (1-a x(1-x))$. Even by choosing a more conservative momentum cutoff such as the Plank scale $M_{p l} \simeq 10^{19} \mathrm{GeV} / \mathrm{c}^{2}$, the resulting mass correction will be 30 orders of magnitude larger than the physical Higgs $\left(m_{\phi}<1 \mathrm{TeV}\right)$. It is possible to soak up this divergence in the bare mass of the Higgs, but due to the magnitude of the correction, $m_{H, 0}$ must be negative and fine tuned to roughly 1 part in $10^{15}$. Furthermore, in this example only the first order (one-loop) correction to the Higgs mass is considered, but this 
fine-tuning must be made at each order in perturbation theory. Though there is nothing formally wrong with doing this, it remains a very unattractive feature of the Standard Model Higgs. This feature has been given the name the "hierarchy problem," and is endemic to all theories which contain fundamental scalar particles.

\subsection{Beyond the Standard Model}

The Standard Model of particle physics as we know it today took roughly the last three-quarters of the twentieth century to be formulated. Beginning in the 1930's, the fundamental building blocks of the model were first identified. By the 1980's, there remained to be measured just 3 free parameters out of the total 18 (21 if neutrinos have mass). Today, the Higgs Boson is the only unmeasured particle predicted by the Standard model. Physicists widely agree, however, that the Standard Model is not the final theory of particle interactions. Inadequacies of the Standard Model usually fall within one of two categories: inelegance or incompleteness.

The most obvious incompleteness of the Standard Model is its lack of inclusion of the forces of gravitation. The reason for this is not due to a lack of imagination on the part of theorists. The force of gravity is some $10^{-36}$ times smaller than the electromagnetic force and $10^{-34}$ times smaller than the electroweak force, making any tests on gravitational models at currently accessible energy scales inadequate.

The Standard Model cannot explain the origin of the fermion and boson masses nor why their mass spectrum is so broad. The lightest quark, the $u$, is some $10^{5}$ times lighter than the top quark. There is also dissatisfaction with the Standard Model's inability to explain why there are exactly three generations of quarks and leptons. Recently, evidence for neutrino oscillations suggesting small neutrino masses has been discovered[57] which would increase the number of SM free parameters from 18 to 21. Neutrino oscillations are not yet accounted for in the Standard Model.

Inelegancies of the Standard Model are usually a consequence of its mathematical formulation. The most obvious inelegance is in its treatment of ultra-violet divergences in the Higgs self-energy corrections (see Section 1.4). Some physicists also believe that the Standard Model is crude in the way it splices together electroweak theory and QCD [75] and for depending on some 18 empirical parameters not including the neutrino masses.

Still, the Standard Model is the only working, renormalizable theory that can account for the existing enormous amounts of experimental data. For all of its inelegance, the Standard Model's ability to account for 3 of the 4 fundamental forces of the universe is one of human-kind's greatest achievements. Physicists agree, however, that the theory is a low energy approximation to a more unified theory of forces and models. Attempting to 
generalize the standard model while avoiding its pitfalls is a focus of research for high-energy experimentalists. The next chapter discusses some examples of theories which go beyond the Standard Model. 


\section{Chapter 2}

\section{Leptoquarks}

In this chapter the properties of leptoquarks are discussed. Theories containing leptoquarklike particles are introduced followed by a presentation of a classification model of leptoquarks based on their possible couplings. The possible leptoquark production and decay modes are discussed in the context of the Tevatron p $\bar{p}$ collider. The details of the leading and next-toleading order theoretical cross section calculation are given followed by presentation of the existing limits on leptoquark masses.

\subsection{Leptoquark Theories}

The inadequacies of the Standard Model serve as motivation for models which predict new physics. Because of the incredible agreement between Standard Model predictions and experiment, many of these theories postulate models in which the Standard Model becomes a low energy approximation. Theories which generalize the Standard Model often contain at lower energies the same invariances, particles and parameters that appear in the Standard Model. Likewise these theories may contain more encompassing invariances at higher energies, a richer particle content, or may predict a finer particle substructure.

Common to many Standard Model extensions is a symmetry between leptons and quarks. Because the Standard Model has benefitted from the relationship between symmetries and conserved quantities, it is common for theorists to attempt to connect the quark phenomenology and lepton phenomenology of the Standard Model. The Standard Model already exploits the equal number of quarks and leptons through the cancellation of anomalies from the leptons with the oppositely signed anomalies from the quarks [64]. Any model which attempts to unify forces via more fundamental couplings is likely to introduce a coupling between

quarks and leptons. If such a coupling exists, then there must be a gauge boson which is the carrier of force between them: The Leptoquark. 
Leptoquarks are hypothetical particles which appear in a number of extensions of the Standard Model as new bosons coupling to a lepton-quark pair [1]. The characteristic feature of leptoquarks is that they are color-triplet particles carrying both baryon and lepton quantum numbers. They are assumed to be scalar (spin 0) or vector (spin 1) particles which can be produced both singly and in pairs. All other particle characteristics, such as weak isospin and electric charge, are model dependent. Like particles of the Standard Model, leptoquark masses are not predicted. In most cases, leptoquarks themselves are not necessarily meant to be a solution to some specific inadequacy of the Standard Model. Rather they are merely an outcome of such theories, several of which will be discussed in the following sections.

\subsubsection{Grand Unified Theories}

The unification of the electromagnetic and electroweak forces by the $S U(2) \times U(1)$ group is a remarkable achievement. It serves as an example that two apparently disparate forces, the electroweak and electromagnetic, can be shown to be manifestations of the same underlying force at a higher energy scale. The success of using gauge field theories to describe particle interactions and the electroweak unification shows promise that all of the forces can perhaps be described by some larger encompassing gauge theory. This larger gauge group, $\mathcal{G}$, would be able to describe the strong, weak, and electromagnetic interactions and be large enough to contain the SM gauge symmetries, i.e.

$$
\mathcal{G} \supset S U(3) \times S U(2) \times U(1)
$$

The idea that at some very high energy scale, $M_{G}$, all of the interactions are mediated with the same coupling strength is the distinguishing feature of GUT models. The electroweak couplings $g$ and $g^{\prime}$ and the strong coupling constant $g_{s}$ are in fact not constant at all but scale with the momentum transfer, $Q$, of an interaction. The difficulty any GUT model must overcome is to relate the electroweak and strong coupling constants, thus unifying the forces.

Figure 2.1 illustrates how the couplings of the $U(1), S U(2)$ and $S U(3)$ groups evolve as a function of energy. At small energies, or large distances, $\alpha_{3}$ asymptotically explodes due to quark confinement signalling the breakdown of QCD perturbation. Electroweak symmetry is still restored at energies around $10^{2} \mathrm{GeV}$, but due to the coupling's logarithmic energy

dependence, it isn't until $10^{15} \mathrm{GeV}$, or $10^{-31} \mathrm{~m}$, that couplings merge to a unified coupling $g_{G}$. At this very high energy scale the interactions would have the same intrinsic strength and therefore there would be nothing to distinguish the leptons from the quarks. In fact, because the particles interact via the same unified coupling, leptons are allowed to transition 


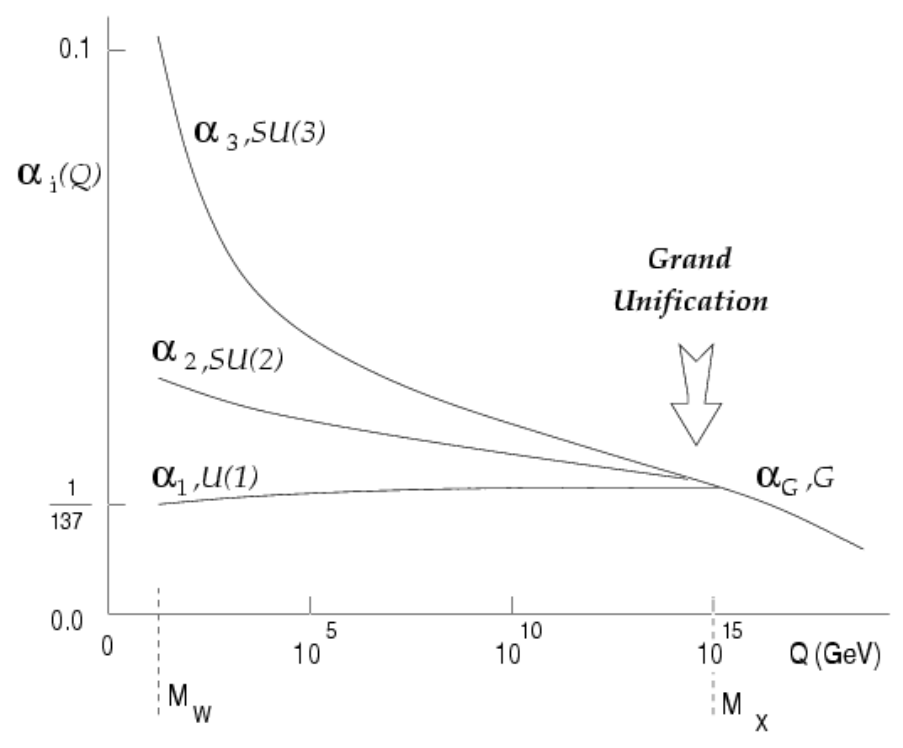

Figure 2.1: The strong, weak and electromagnetic couplings as a function of energy (from Kambara [76]).

to quarks and vice versa.

By 1973, Howard Georgi and Sheldon Glashow had worked out the simplest representation of such a GUT model in which the $S U(3) \times S U(2) \times U(1)$ combination of gauge theories in the Standard Model are components of a $S U(5)$ gauge symmetry [60,61]. Analogous to the $S U(3)$ triplets of colored quarks or the weak $S U(2)$ doublet, the $S U(5)$ symmetry group is represented by traceless 5 element vectors which form the basis of the group representation. The electric charge is a generator of the $S U(5)$ group which enables an account of charge quantization. The $S U(5)$ unified model introduces 12 new gauge bosons $X$ and $Y$ having charges $q= \pm 4 / 3, \pm 1 / 3$ respectively which exist only at energies above $10^{15} \mathrm{GeV}$. The interactions mediated by $X$ and $Y$ violate conservation of baryon number, $B$, allowing the proton to decay to a neutron. Since the new gauge bosons are exchanged between the leptons and quarks at unreachable energy scales, their existence can only be detected indirectly. The prediction of proton decay is thus an important feature of the $S U(5)$ model because it allows the model to be tested experimentally.

The $S U(5)$ model is elegant and has novel features including the prediction of charge quantization, but is no longer considered a viable theory of particle interactions. The lifetime of a proton has an experimental upper limit of $>10^{31}$ years [88], and the theory's predictions when extrapolated to accessible energy scales are in poor agreement with experiment. The model also does not avoid the defect of the Hierarchy Problem, which as pointed out 
in Section 1.4 cannot be avoided without cancelling the divergences with explicit partner particles or by avoiding point-like couplings to scalar particles as is the case in composite models.

Another renormalizable GUT theory postulating the existence of leptoquark-like particles is the Pati-Salam Color Symmetric Model [91]. In this SM extension, the $S U(3)_{c}$ color group is extended to a $S U(4)_{c}$ group where lepton number $L$ is the fourth color thus unifying baryonic and leptonic matter. The quarks form a quartet having quantum numbers of isospin, strangeness $S$, and charm $C$, giving a total global symmetry

$$
S U(4)_{L} \times S U(4)_{R} \times S U(4)_{c}
$$

The gauge bosons mediating the forces between the quarks and leptons are vector particles having a non-chiral coupling to the leptons and quarks. In the Pati-Salam model, the leptoquarks are not restricted to couple uniquely within a lepton-quark generation [101]. These inter-generation couplings give rise to FCNC decays such as $B_{s} \rightarrow e \mu$ and $B^{0} \rightarrow \tau \mu$ (see Figure 2.10). Searches for Pati-Salam leptoquarks at CDF in Run I resulted in mass constraints with a $95 \%$ C.L. of $M_{L Q}>19.3 \mathrm{TeV}$ for a leptoquark coupling to $e, \mu$ and $M_{L Q}>20.4 \mathrm{TeV}$ for a leptoquark coupling to $e, \tau[103]$.

\subsubsection{Dynamic Symmetry Breaking and Composite Models}

In dynamic symmetry breaking, or technicolour, models the Higgs electroweak symmetry breaking is replaced by a dynamical symmetry breaking [102] from a confining strong force at an energy scale $\Lambda \sim G_{F}^{-1 / 2}$ [55]. The need for a Higgs Model scalar vacuum expectation value is obviated by a strong-coupling, confining, non-abelian gauge theory:

$$
G_{T C} \times S U(3)_{c} \times S U(2)_{L} \times U(1)
$$

The symmetry breaking of the technicolour group results in Goldstone bosons which may have leptoquark properties. The estimate for their mass is $\sim \mathcal{O}\left(200 \mathrm{GeV} / \mathrm{c}^{2}\right)$ for $\Lambda_{T C} \sim$ $1 \mathrm{TeV} / \mathrm{c}^{2}[92]$.

The concept of endowing masses to the fermions and gauge bosons via a dynamical symmetry breaking has led to the formulation of a number of composite theories $[22,55$, $93,33]$ which theorize a low-energy, strong-confining $S U(2)_{L}$ gauge. In composite models fermions and gauge bosons are composite states of a set of fundamental fields which interact through a confining strong force. The behavior of weak interactions at currently accessible energy levels are thus analogous to the interactions of strongly bound meson states. The exact gauge group structure is model dependent. In the simplest model by Abbott and Farhi (AF) the gauge lagrangian is formally identical to the standard electroweak GSW 
model [22]. All left-handed fermions are $S U(2)_{L}$ singlets of this strong electroweak gauge force, while right-handed particles are still point-like. Left-handed fermions are bound states of the left-handed fundamental fields called "preons". Leptoquarks appear in the AF model as bound states of preons [73].

Other strong confining gauge groups are possible. For example there is a double confining $S U(2)_{L} \times S U(2)_{R}$ technicolour gauge theory $[33,34]$ which has only fundamental fermions and different confinement scales. In strong confining gauge theories leptoquarks appear as Goldstone bosons and can be naturally light, having a mass from a few $100 \mathrm{GeV} / \mathrm{c}^{2}$ to a few $\mathrm{TeV} / \mathrm{c}^{2}[92]$.

\subsubsection{SUSY}

Another model which generalizes the SM and avoids the hierarchy problem is "supersymmetry" (SUSY) which refers to a fermion-boson symmetry for which each fermion(boson) shares the same coupling with a boson(fermion) partner. This symmetry, denoted $R$ symmetry, is apparently broken since fermionic mass scales are not identical. By postulating the existence of supersymmetric partners to the existing SM particles, it is possible to cancel divergences in the theory to all orders of perturbation, even if the mass splitting between the scalar-vector partners occurs at the weak scale [52]. There are several models which employ supersymmetry to solve the hierarchy problem, the most common being the minimal supersymmetric extension of the Standard Model (MSSM) which postulates a minimum addition of supersymmetric partners plus two spin $\pm 1 / 2$ Higgs doublets. MSSM predicts SUSY particles, or "sparticles", to exist at energies below $\mathcal{O}(1 \mathrm{TeV})$ and in some cases substantially lighter [88].

In the context of high energy colliders, the consequences of $R$-parity conservation are significant. The $R$-parity of a particle is given by the spin $j$, baryon number $B$ and lepton number $L$ :

$$
R=(-1)^{3 B+L+2 j}
$$

hence giving ordinary particles $R=+1$ and supersymmetric particles $R=-1$. If $R$ parity is conserved, then at today's particle colliders, sparticles would be produced in pairs and there would be a lightest-state stable sparticle unable to decay to non-supersymmetric particles. If $R$-parity is not conserved, then sparticles are allowed to decay completely into non-supersymmetric particles. Such a decay would mean that sparticles could have leptoquark-like properties as would be the case for a squark, $\tilde{q}$ decaying to a lepton-quark pair, e.g. $\tilde{q} \rightarrow \ell q$. 


\subsection{The Buchmüller-Rückle-Wyler Leptoquark Classification}

A model generalizing the possible leptoquark states consistent with the $S U(3) \times S U(2) \times$ $U(1)$ gauge group is that of the Buchmüller-Rückle-Wyler (BRW) [35]. In the BRW classification scheme, leptoquarks are categorized according to all of the possible lepton-quark couplings, as shown in Table 2.1. The classification comprises a possible seven scalar $(S)$ and seven vector $(V)$ leptoquark models, denoted in the first column. The $L$ and $R$ superscripts denote whether the leptoquark couples to a left- or right-handed lepton, and the subscript denotes the weak isospin. Table 2.1 is also symmetric with respect to charge conjugation and the particles can be globally replaced with the corresponding first- and third-generation leptons and quarks. The charge, $Q$ is given in units of electron charge, e, and the fermion number $F$ is given by $F=L+3 B$ where $L=+1$ for electrons and $B=+1 / 3$ for quarks. The BRW models assume that the leptoquarks are chiral, and thus can only couple to left- or right-handed leptons. Additionally both the lepton number and baryon number must be conserved separately in leptoquark decays. These restrictions on the leptoquark lepton-quark coupling allow the leptoquarks to have a mass as light as $\sim 100 \mathrm{GeV} / \mathrm{c}^{2}$ while avoiding conflicts with rapid proton decay and FCNC [67]. The unknown coupling of the lepton to the lepton-quark is denoted by $\lambda$. Finally, the last column shows the possible squark assignments in the MSSM theory with broken $R$-parity. 


\begin{tabular}{|c|c|c|c|c|c|}
\hline Model & $\mathbf{F}$ & Charge Q & $\beta$ & Coupling & Squark \\
\hline$S_{0}^{L}$ & 2 & $-1 / 3$ & 0.5 & $\lambda_{L}\left(\mu_{L}^{-} c\right),-\lambda_{L}(\nu s)$ & $\tilde{d}_{R}$ \\
\hline$S_{0}^{R}$ & 2 & $-1 / 3$ & 1 & $\lambda_{R}\left(\mu_{R}^{-} c\right)$ & \\
\hline$\tilde{S}_{0}^{R}$ & 2 & $-4 / 3$ & 1 & $\lambda_{R}\left(\mu_{R}^{-} s\right)$ & \\
\hline \multirow[t]{2}{*}{$S_{1 / 2}^{L}$} & 0 & $-5 / 3$ & 1 & $\lambda_{L}\left(\mu_{L}^{-} \bar{c}\right)$ & \\
\hline & & $-2 / 3$ & 0 & $\lambda_{L}(\nu \bar{c})$ & \\
\hline \multirow[t]{2}{*}{$S_{1 / 2}^{R}$} & 0 & $-5 / 3$ & 1 & $\lambda_{R}\left(\mu_{R}^{-} \bar{c}\right)$ & \\
\hline & & $-2 / 3$ & 1 & $-\lambda_{R}\left(\mu_{R}^{-} \bar{s}\right)$ & \\
\hline \multirow[t]{2}{*}{$\tilde{S}_{1 / 2}^{L}$} & 0 & $-2 / 3$ & 1 & $\lambda_{L}\left(\mu_{L}^{-} \bar{s}\right)$ & $\overline{\tilde{c}}_{L}$ \\
\hline & & $+1 / 3$ & 0 & $-\lambda_{L}(\nu \bar{s})$ & $\overline{\tilde{s}}_{L}$ \\
\hline \multirow[t]{3}{*}{$S_{1}^{R}$} & 2 & $-4 / 3$ & 1 & $-\sqrt{2} \lambda_{L}\left(\mu_{L}^{-} s\right)$ & \\
\hline & & $-1 / 3$ & 0.5 & $-\lambda_{L}\left(\mu_{L}^{-} c\right),-\lambda_{L}(\nu s)$ & \\
\hline & & $+2 / 3$ & 0 & $\sqrt{2} \lambda_{L}(\nu c)$ & \\
\hline$V_{0}^{L}$ & 0 & $-2 / 3$ & 0.5 & $\lambda_{L}\left(\mu_{L}^{-} s\right), \lambda_{L}(\nu \bar{c})$ & \\
\hline$V_{0}^{R}$ & 0 & $-2 / 3$ & 1 & $\lambda_{R}\left(\mu_{R}^{-} \bar{s}\right)$ & \\
\hline$\tilde{V}_{0}^{R}$ & 0 & $-5 / 3$ & 1 & $\lambda_{R}\left(\mu_{R}^{-} \bar{c}\right)$ & \\
\hline \multirow[t]{2}{*}{$V_{1 / 2}^{L}$} & 2 & $-4 / 3$ & 1 & $\lambda_{L}\left(\mu_{L}^{-} s\right)$ & \\
\hline & & $-1 / 3$ & 0.5 & $\lambda_{L}(\nu s)$ & \\
\hline \multirow[t]{2}{*}{$V_{1 / 2}^{R}$} & 2 & $-4 / 3$ & 1 & $\lambda_{R}\left(\mu_{R}^{-} s\right)$ & \\
\hline & & $-1 / 3$ & 1 & $\lambda_{R}\left(\mu_{R}^{-} c\right)$ & \\
\hline \multirow[t]{2}{*}{$\tilde{V}_{1 / 2}^{L}$} & 2 & $-1 / 3$ & 1 & $\lambda_{L}\left(\mu_{L}^{-} c\right)$ & \\
\hline & & $+2 / 3$ & 0 & $\lambda_{L}(\nu c)$ & \\
\hline \multirow[t]{3}{*}{$V_{1}^{L}$} & 0 & $-5 / 3$ & 1 & $\sqrt{2} \lambda_{L}\left(\mu_{L}^{-} \bar{c}\right)$ & \\
\hline & & $-2 / 3$ & 0.5 & $-\lambda_{L}\left(\mu_{L}^{-} \bar{s}\right), \lambda_{L}(\nu \bar{c})$ & \\
\hline & & $+1 / 3$ & 0 & $\sqrt{2} \lambda_{L}(\nu \bar{s})$ & \\
\hline
\end{tabular}

Table 2.1: A classification of leptoquarks in the Buchmüller-Rückl-Wyler leptoquark scheme [35] adopted from [94]. Shown is the leptoquark fermion number $F=3 B+L$, the electric charge $Q$ in units of $e$, the branching ratio $\beta$, and the lepton-quark couplings. The last column shows the possible squark assignments to the leptoquark states in the MSSM with broken R-parity. The classification assumes conjugation symmetry and the ability to globally replace the second-generation particles with first- or third-generation particles. 
In this thesis, we search for leptoquarks in the $\mu q \mu q$ and $\mu q \nu q$ channels, corresponding to the BRW leptoquarks $\tilde{S}_{1 / 2}^{L}$ and $S_{0}^{L}$ respectively. Although the coupling assignments for these scalar leptoquarks correspond to the MSSM squarks $\tilde{d}_{R}$ and $\overline{\tilde{c}}_{L}$, the searches in this analysis are not squark searches because the squark production cross sections are not the same as the leptoquark production cross section as discussed in Section 2.5.4.

\subsection{Leptoquark Production}

At the Tevatron, leptoquarks can be directly produced both singly and in pairs:

$$
\begin{array}{ll}
p+\bar{p} & \rightarrow L Q+\overline{L Q}+X \\
p+\bar{p} & \rightarrow \quad L Q+\bar{\ell}+X
\end{array}
$$

The Feynman diagrams corresponding to single leptoquark production are shown in Figure 2.2 .
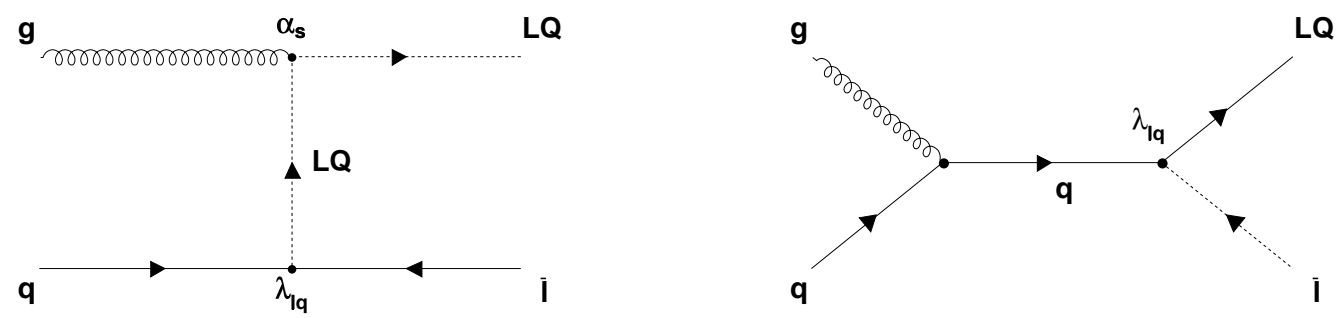

Figure 2.2: Feynman diagrams of the parton level process $g q \rightarrow L Q \ell$. The parameter $\lambda_{\ell q}$ represents the coupling between the lepton and quark.

The production cross-section for singly produced leptoquarks is quadratically dependent on the leptoquark fermionic couplings $\lambda_{\ell q}$ between the lepton and quark. Searches for singly produced leptoquarks will thus always lead to combined bounds on the leptoquark mass and the coupling constant $\lambda_{\ell q}$. The consequence is that leptoquark production cross sections are bounded to be very small in the mass range up to $\mathcal{O}(1 \mathrm{TeV})$ [31]. For example, in the case that $M_{L Q_{s}}=200 \mathrm{GeV} / \mathrm{c}^{2}$ and $\lambda_{\ell q}=e / 10$, one finds $\sigma\left[p \bar{p} \rightarrow L Q_{s} \bar{\ell}\right] \sim 4 \times 10^{-3}$ $\mathrm{pb}$ [78]. Thus at the current high-energy hadron colliders such as the Tevatron, singlyproduced leptoquarks have a negligible cross section. Theoretical limits on the production cross section of singly-produced leptoquarks at the Run I and Run II Tevatron have been calculated [53]. 
There are three production processes possible for pair-produced leptoquarks. They are:

$$
\begin{aligned}
& q g \rightarrow L Q \overline{L Q} \\
& q \bar{q} \rightarrow L Q \overline{L Q} \\
& g g \rightarrow L Q \overline{L Q}
\end{aligned}
$$

The process in Equation 2.3 contributes to the partonic cross-section in order $\alpha_{s}^{3}$ and is included only in the Next-to-Leading order calculation as will be discussed in Section (2.5.3). The most common mechanism for leptoquark pair production at high-energy hadron colliders is both quark-antiquark annihilation (Equation 2.4) and gluon fusion (Equation 2.5). Figures 2.3 and 2.4 show the leading order contributions to the partonic pair production crosssection. At the LHC, the predominant mechanism is gluon fusion, whereas the predominant mechanism at the Tevatron is quark-antiquark annihilation, with the largest contribution to the cross section coming from the $s$-channel.

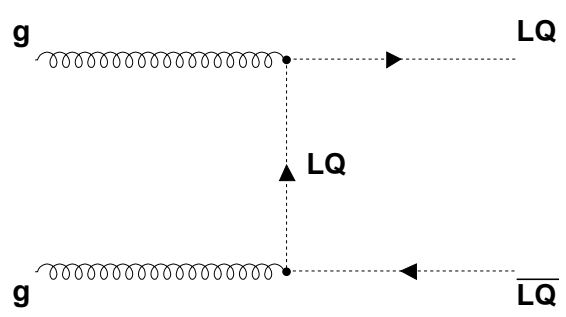

(1)

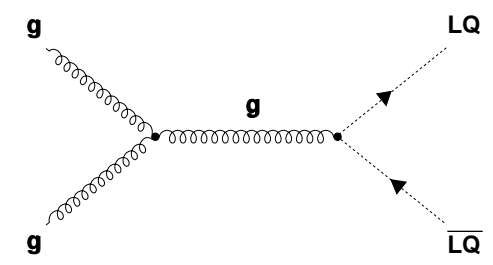

(3)

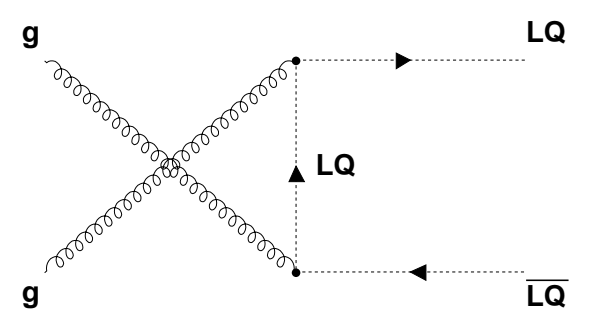

(2)

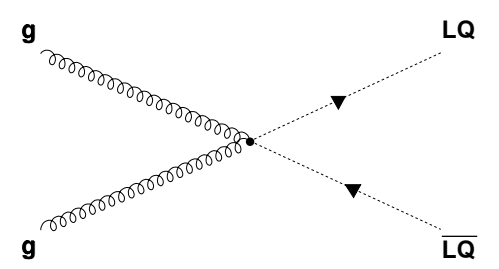

(4)

Figure 2.3: Feynman diagrams of the parton level process $g g \rightarrow L Q \overline{L Q}$. 


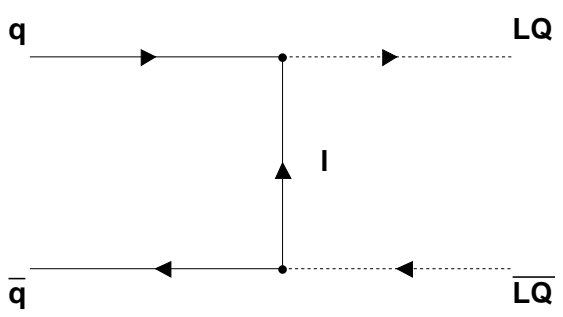

(5)

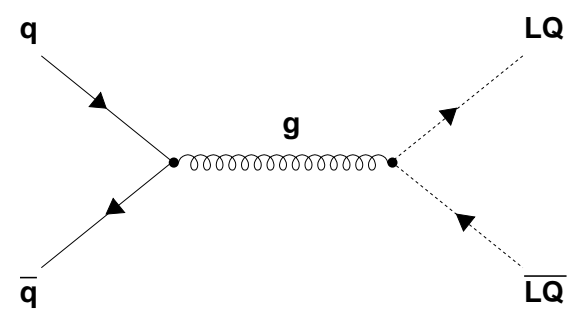

(6)

Figure 2.4: Feynman diagrams of the parton level process $q \bar{q} \rightarrow L Q \overline{L Q}$ (5-6).

The effective Lagrangian describing scalar and vector leptoquark interactions with gluons is:

$$
\mathscr{L}=\mathscr{L}_{S}^{g}+\mathscr{L}_{V}^{g}
$$

where

$$
\begin{aligned}
\mathscr{L}_{S}^{g}= & \sum_{\text {scalars }}\left\{\left(\mathcal{D}_{i j}^{\mu} \Phi^{j}\right)^{\dagger}\left(\mathcal{D}_{\mu}^{i k} \Phi_{k}\right)-M_{S}^{2} \Phi^{i^{\dagger}} \Phi_{i}\right\}, \\
\mathscr{L}_{V}^{g}= & \sum_{\text {vectors }}\left\{-\frac{1}{2} G_{\mu \nu}^{i \dagger} G_{i}^{\mu \nu}+M_{V}^{2} \Phi_{\mu}^{i^{\dagger}} \Phi_{i}^{\mu}-\right. \\
& \left.i g_{s}\left[\left(1-\kappa_{G}\right) \Phi_{\mu}^{i \dagger} t_{i j}^{a} \Phi_{\nu}^{j} \xi_{a}^{\mu \nu}+\frac{\lambda_{G}}{M_{V}^{2}} G_{\sigma \mu}^{i}{ }^{\dagger} t_{i j}^{a} G_{\nu}^{j \mu} \xi_{a}^{\mu \sigma}\right]\right\} .
\end{aligned}
$$

The strong coupling constant is denoted by $g_{s}, t_{a}$ are the generators of $S U(3)_{c}, M_{S}\left(M_{V}\right)$ is the scalar (vector) leptoquark mass, and $\kappa_{G}$ and $\lambda_{G}$ are the anomalous coupling constants. The gluon field strength tensor and vector leptoquark field strength tensor are given by respectively [31]:

$$
\begin{aligned}
& \xi_{\mu \nu}^{a}=\partial_{\mu} A_{\nu}^{a}-\partial_{\nu} A_{\mu}^{a}+g_{s} f^{a b c} A_{\mu b} A_{\nu c} \\
& \xi_{\mu \nu}^{i}=\mathcal{D}_{\mu}^{i k} \Phi_{\nu k}-\mathcal{D}_{\nu}^{i k} \Phi_{\mu k}
\end{aligned}
$$

Finally, the covariant derivative is given by:

$$
\mathcal{D}_{\mu}^{i j}=\partial_{\mu} \delta^{i j}-i g_{s} t_{a}^{i j} A_{\mu}^{a}
$$

where $A_{\mu}^{a}$ is the local transformation field containing a non-Abelian tensor term.

The gluon-leptoquark interactions are determined by the non-Abelian $S U(3)_{c}$ gauge- 
symmetry of scalar QCD, making theoretical calculation of the pair-production cross-section dependent on the leptoquark quantum numbers and independent of the lepton-quark coupling, $\lambda_{l q}$ [1]. For the case of scalar leptoquarks, this means that the production cross-section can be calculated as a function of leptoquark mass only. For the case of vector leptoquarks, the production cross-section depends on the values of the anomalous couplings. These couplings are related to the magnetic moment $\mu_{V}$ and electric quadrapole moment $q_{V}$ by [31]

$$
\begin{aligned}
\mu_{V} & =\frac{g_{s}}{2 M_{V}}\left(2-\kappa_{G}+\lambda_{G}\right), \\
q_{V} & =-\frac{g_{s}}{M_{V}^{2}}\left(1-\kappa_{G}-\lambda_{G}\right),
\end{aligned}
$$

respectively. There are no direct bounds on the vector leptoquark anomalous couplings, but they are assumed to be real numbers. There are typically two choices to constrain the couplings:

1. Yang-Mills type coupling: $\kappa_{G}=\lambda_{G}=0$

2. Minimal Vector type coupling: $\kappa_{G}=1, \lambda_{G}=0$

The Minimal Vector type coupling has a smaller calculated cross section than the Yang-Mills type coupling and both have larger cross sections than scalar leptoquarks. In this analysis a search is performed for scalar leptoquarks only.

\subsection{Leading Order Cross Section}

The differential leading order partonic scattering cross-section for quark-antiquark annihilation and gluon fusion are given by [2]:

$$
\begin{aligned}
\frac{d \sigma_{L O}}{d \cos \theta}\left[q \bar{q} \rightarrow L Q_{S} \overline{L Q}_{S}\right]= & \frac{\alpha_{s}^{2} \pi}{18 \hat{s}} \beta^{3} \sin ^{2} \theta \\
\frac{d \sigma_{L O}}{d \cos \theta}\left[g g \rightarrow L Q_{S} \overline{L Q}_{S}\right]= & \frac{\alpha_{s}^{2} \pi}{6 \hat{s}}\left\{\frac{1}{32}\left[25+9 \beta^{2} \cos ^{2} \theta-18 \beta^{2}\right]\right. \\
& \left.-\frac{1}{16} \frac{\left(25-34 \beta^{2}+9 \beta^{4}\right)}{1-\beta^{2} \cos ^{2} \theta}+\frac{\left(1-\beta^{2}\right)^{2}}{\left(1-\beta^{2} \cos ^{2} \theta\right)^{2}}\right\}
\end{aligned}
$$

where $\sqrt{\hat{s}}$ is the invariant energy of the subprocess, $\alpha_{s}$ is the strong coupling constant, and $\beta=\left(1-4 M_{L Q}^{2} / \hat{s}\right)^{1 / 2}$. In the integral form they appear as:

$$
\begin{aligned}
\sigma_{L O}\left[q \bar{q} \rightarrow L Q_{S} \overline{L Q}_{S}\right] & =\frac{\alpha_{s}^{2} \pi}{\hat{s}} \frac{2}{27} \beta^{2} \\
\sigma_{L O}\left[g g \rightarrow L Q_{S} \overline{L Q}_{S}\right] & =\frac{\alpha_{s}^{2} \pi}{96 \hat{s}}\left\{\beta\left(41-31 \beta^{2}\right)+\left(18 \beta^{2}-\beta^{4}-17\right) \log \frac{1+\beta}{1-\beta}\right\}
\end{aligned}
$$


Analogous to equations 2.14 and 2.15, the vector leptoquark leading-order scattering cross-sections are given by [3]:

$$
\begin{aligned}
\sigma_{L O}\left[q \bar{q} \rightarrow L Q_{V} \overline{L Q}_{V}\right]= & \frac{\alpha_{s}^{2} \pi}{27 \hat{s}} \frac{\beta^{3}}{1-\beta^{2}}\left[23-3 \beta^{2}+\frac{4}{1+\beta^{2}}\right] \\
\sigma_{L O}\left[g g \rightarrow L Q_{V} \overline{L Q}_{V}\right]= & \frac{\alpha^{2} \pi}{24 \hat{s}} \frac{1}{\left(1-\beta^{2}\right)}\left\{\beta\left(\frac{523}{4}-90 \beta^{2}+\frac{93}{4} \beta^{4}\right)\right. \\
& \left.-\frac{3}{4}\left(65-83 \beta^{2}+19 \beta^{4}-\beta^{6}\right) \log \frac{1+\beta}{1-\beta}\right\},
\end{aligned}
$$

\subsection{Next-to-Leading Order Cross Section}

The Next-to-Leading Order (NLO) cross section for pair produced leptoquarks at the Tevatron has been calculated [1]. The NLO calculation includes QCD radiative corrections to equation (2.14). A brief outline of the contributing processes included in the calculation follows.

\subsubsection{Virtual Corrections}

Virtual corrections to the leptoquark partonic cross section are carried out to order $\alpha_{s}$. They involve self-energy diagrams, initial/final-state interactions, and vertex corrections for quarks, gluons and leptoquarks. A sample of Feynman diagrams for these processes contributing to the amplitude in Equation (2.5) is shown in Figure 2.5. The amplitudes from

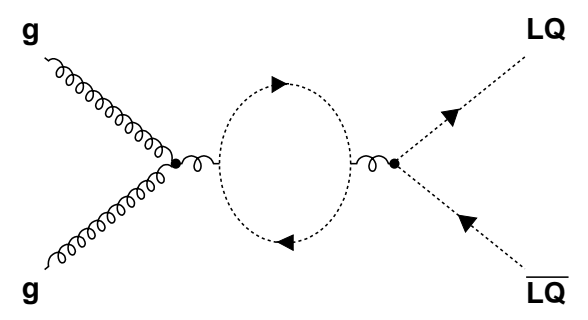

(7)

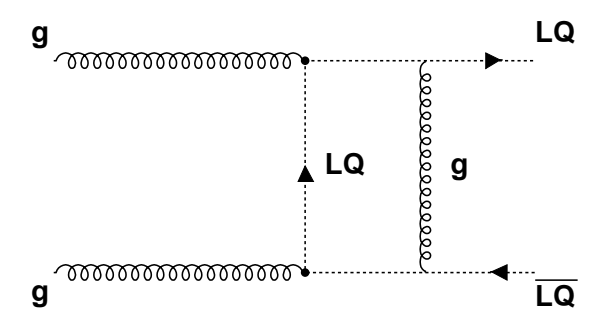

(8)

Figure 2.5: Example Feynman diagrams for virtual corrections involving self-energy (7) and vertex correction (8).

virtual corrections are calculated in the Feynman gauge. The ultraviolet (UV), infrared (IR), and collinear mass (M) singularities are removed using n-dimensional regularization[29] and the renormalization is carried out in the modified minimal-subtraction scheme $(\overline{M S})$. 


\subsubsection{Bremsstrahlung of Gluons}

The momentum assignment from the lowest order gluon-gluon-fusion process is given by:

$$
g\left(k_{1}\right)+g\left(k_{2}\right) \rightarrow Q\left(p_{1}\right)+\bar{Q}\left(p_{2}\right)
$$

It is convenient to choose the variables:

$$
\begin{aligned}
s & =2 k_{1} \cdot k_{2} \\
t_{1} & \equiv t-m^{2}=\left(k_{1}-p_{1}\right)^{2}-m^{2} \\
u_{1} & \equiv u-m^{2}=\left(k_{1}-p_{2}\right)^{2}-m^{2}
\end{aligned}
$$

Higher order gluon-gluon-fusion processes contain gluons emitted off colored lines and scalar vertices. Two examples are shown in Figure 2.6. To calculate corrections to the partonic cross-section from gluon bremsstrahlung, a gluon is added to the final state in equation (2.18):

$$
g\left(k_{1}\right)+g\left(k_{2}\right) \rightarrow Q\left(p_{1}\right)+\bar{Q}\left(p_{2}\right)+g\left(k_{3}\right)
$$

There are a total of 11 gluon radiation diagrams which contribute to the cross-section to to order $\alpha_{s}$. A complete discussion of how to compute these contributions can be found in [29]. The gluon emission cross-section is calculated using the phase-space slicing method

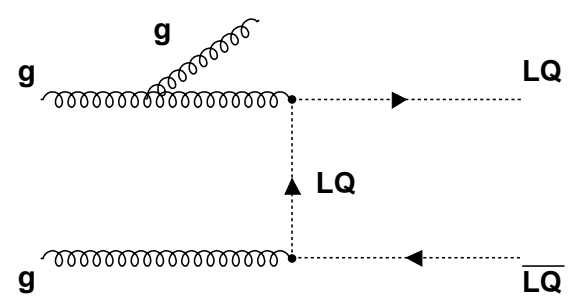

(9)

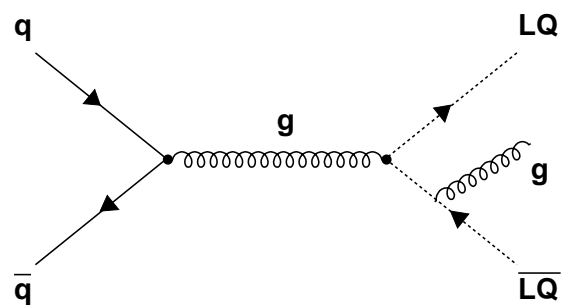

$(10)$

Figure 2.6: Generic Feynman diagrams for gluon radiation.

[29]. Soft and hard gluon radiation is separated by imposing a cutoff $\Delta$ for the invariant mass of the final-state leptoquark-gluon system and any remaining $\Delta$-dependence after both contributions are added will disappear in the limit $\Delta \rightarrow 0$. Infrared singularities are canceled when soft gluon emission is added to the virtual corrections. Any remaining initial-state singularities are absorbed into the renormalization of the parton densities, defined again in 
the $\overline{M S}$ scheme.

\subsubsection{Compton Process}

These production mechanisms involve contributions from gluon-quark collisions and are present only in order $\alpha_{s}^{3}$. Feynman diagrams showing these processes is shown in Figure 2.7.

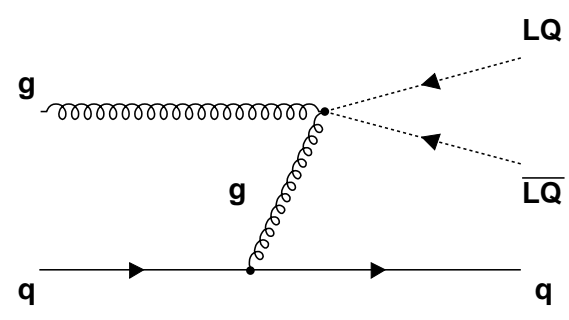

$(11)$

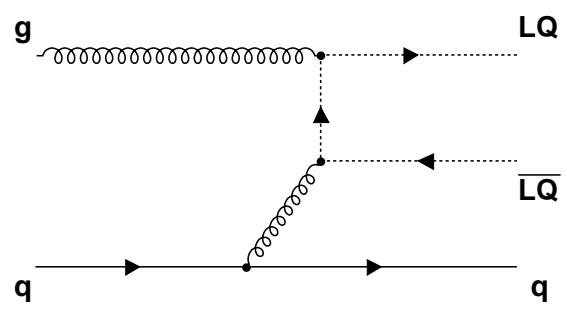

$(12)$

Figure 2.7: Generic Feynman diagrams for Compton scattering.

\subsubsection{Numerical Calculation}

Leptoquarks having non-pointlike structures like those predicted in composite models are not expected below energy scales of $\sim 1 \mathrm{TeV}$ [92]. Therefore, for the purposes of calculating a production cross section, leptoquarks are assumed to be pointlike particles. To determine the total NLO cross-section for pair produced scalar leptoquarks, the contributions from the QCD corrections are folded into the LO partonic cross-section and perturbatively expanded in terms of scaling functions $f_{i j}$ :

$$
\hat{\sigma}_{i j}\left(s, M_{L Q}^{2}\right)=\frac{\alpha_{s}^{2} \mu^{2}}{M_{L Q}^{2}}\left[f_{i j}^{(0)}(\eta)+4 \pi \alpha_{s} \mu^{2} \times\left\{f_{i j}^{(1)}\left(\eta, r_{t}\right)+\bar{f}_{i j}^{(1)}(\eta) \log \frac{\mu^{2}}{M_{L Q}^{2}}\right\}\right] \text {, }
$$

where $i, j=g, q, \bar{q}$ denote the initial-state partons and $\mu$ is the renormalization and factorization scale $\mu=\mu_{R}=\mu_{F}$. The scaling functions in equation (2.23) depend on the invariant parton energy $\sqrt{\hat{s}}$ and leptoquark mass $M_{L Q}$ through $\eta=\hat{s} / 4 M_{L Q}^{2}-1$ and on $r_{t}=m_{\text {top }} / M_{L Q}$, the mass ratio of the top quark and leptoquark.

The numerical values for the total NLO cross-section are determined by including the gluon-gluon and gluon-quark initial states to the LO partonic cross-section and then inte- 
grating the resulting luminosities $\tau d \mathscr{L} / d \tau$ :

$$
\sigma[p \bar{p} \rightarrow L Q+\overline{L Q}+X]=\sum_{i, j=g, q, \bar{q}} \int d \tau \frac{d \mathscr{L}^{i j}}{d \tau} \hat{\sigma} \hat{s}
$$

where $\tau=\hat{s} / s$. The parton density parameterization used in the numerical analysis is CTEQ4M [32], and the top quark mass is set to $m_{t}=175 \mathrm{GeV} / \mathrm{c}^{2}$. The normalization scale is chosen to be $\mu=M_{L Q}$ and the theoretical error is determined using $\mu=2 M_{L Q}$ and $\mu=M_{L Q} / 2$ as upper and lower bounds. The values of $K=\sigma_{N L O} / \sigma_{L O}$ for this scale evaluated in the leptoquark mass range between $150 \leq M_{L Q} / \mathrm{GeV} / \mathrm{c}^{2} \leq 250$ vary only between 1.20 and 1.08 [1]. This demonstrates that the perturbative expansion (2.23) converges reliably. It is also interesting to note that the NLO cross-section results nearly coincide with those of squark-antisquark production in the limit of large gluino mass [67]. Because scalar leptoquarks and squarks have identical couplings to gluons, the gluon fusion contributions to their production cross sections are identical. The quark-antiquark annihilation contributions are also identical for the $s$-channel gluon-exchange shown in Figure 2.4(6). However a difference arises in the $t$-channel Feynman diagram, Figure 2.4(5). For leptoquark production, a massless lepton is exchanged in the $t$-channel and the diagram is proportional to the square of the unknown coupling $\lambda_{\ell q}$ whereas the squark exchanges a massive gluino $\tilde{g}$ with strong $q \tilde{q} \tilde{g}$ coupling.

Figure 2.8 shows the cross section as a function of factorization scale for a scalar leptoquark mass of $220 \mathrm{GeV} / \mathrm{c}^{2}$ at the Run II Tevatron. In the LO case, the cross-section is heavily dependent on the factorization scale. A change of scale from $\mu=2 M_{L Q}$ to $\mu=M_{L Q} / 2$ increases the cross section by $100 \%$. This scale dependence is reduced dramatically by the QCD corrections in the NLO case, varying by only $30 \%$ within the same factorization range.

The values for the LO and NLO theoretical cross-section for a range of scalar leptoquark masses is shown in Table 2.2. Figure 2.9 shows the theoretical cross sections for Tevatron energies in Run I and Run II. The NLO cross sections for $\sqrt{s}=1960 \mathrm{GeV}$ are used in this

\begin{tabular}{|c|cc|cc|c|cc|c|}
\hline$M_{L Q}$ & \multicolumn{2}{|c|}{$\sigma_{g g}(\mathrm{pb})$} & \multicolumn{2}{c|}{$\sigma_{q \bar{q}}(\mathrm{pb})$} & $\sigma_{g q}(\mathrm{pb})$ & \multicolumn{2}{|c|}{$\sigma_{\text {tot }}(\mathrm{pb})$} & $\mathrm{K}$ \\
$\left(\mathrm{GeV} / \mathrm{c}^{2}\right)$ & $\mathrm{LO}$ & $\mathrm{NLO}$ & $\mathrm{LO}$ & $\mathrm{NLO}$ & $\mathrm{NLO}$ & $\mathrm{LO}$ & $\mathrm{NLO}$ & \\
\hline 100 & 6.03 & 10.9 & 5.93 & 5.66 & -0.332 & 12.0 & 16.3 & 1.36 \\
140 & 0.617 & 1.20 & 1.31 & 1.26 & -0.057 & 1.92 & 2.40 & 1.25 \\
180 & 0.0904 & 0.184 & 0.356 & 0.349 & -0.012 & 0.446 & 0.521 & 1.17 \\
220 & 0.0162 & 0.0342 & 0.107 & 0.108 & -0.00259 & 0.123 & 0.139 & 1.13 \\
260 & 0.00327 & 0.00708 & 0.0345 & 0.0346 & -0.000627 & 0.0378 & 0.0411 & 1.09 \\
300 & 0.000716 & 0.00156 & 0.0113 & 0.0115 & -0.000157 & 0.0121 & 0.0129 & 1.07 \\
\hline
\end{tabular}

Table 2.2: Theoretical scalar leptoquark cross-sections for $p \bar{p}$ collisions at $\sqrt{s}=1.96 \mathrm{TeV}$. 


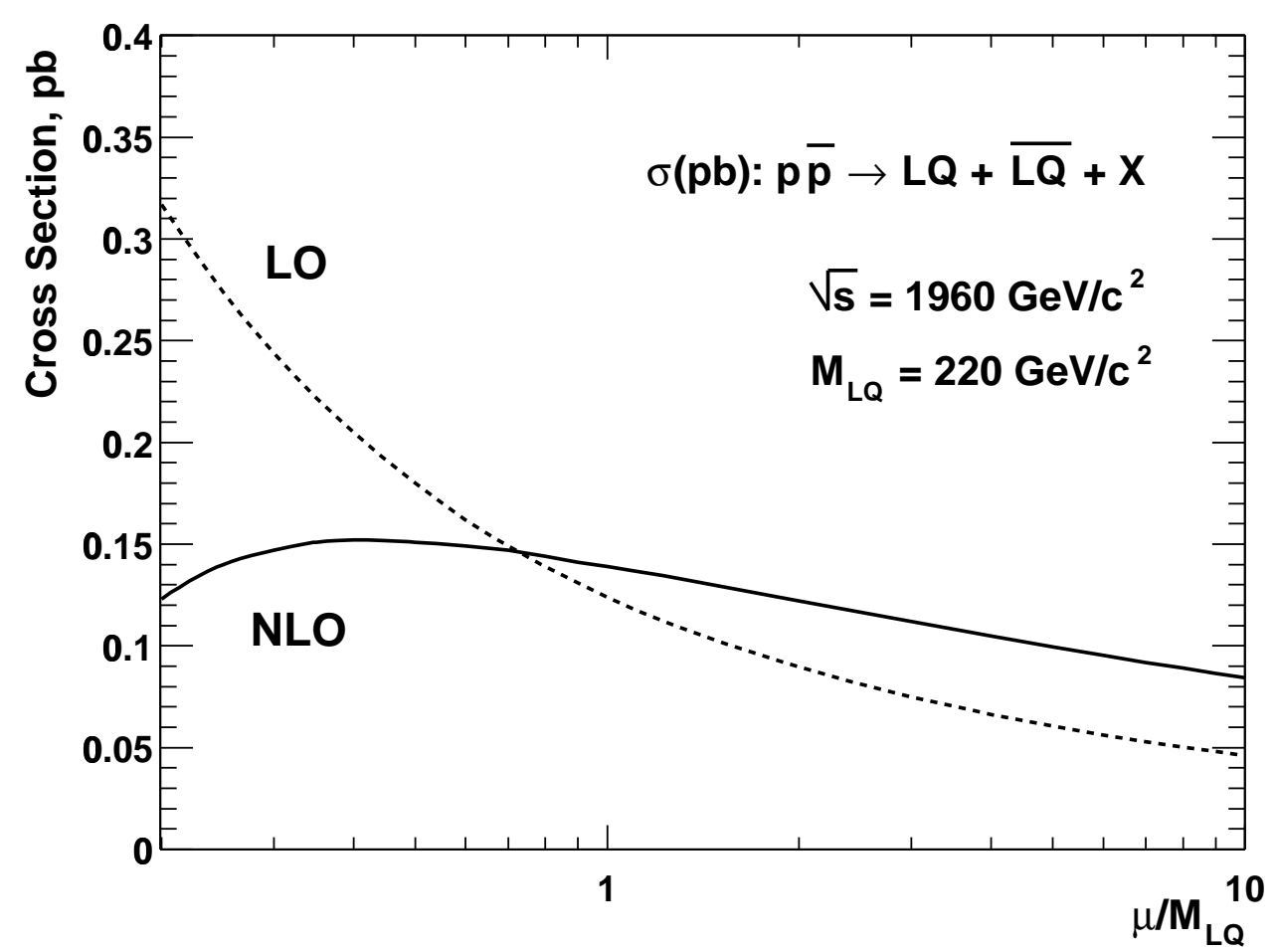

Figure 2.8: The theoretical scalar leptoquark cross section as a function of factorization/normalization scale at the Run II Tevatron.

analysis to set upper limits on the scalar leptoquark mass.

\subsection{Leptoquark Decay}

Leptoquark theories are motivated to restrict the properties of leptoquarks due to experimental limits. For example, due to experimental limits on helicity-suppressed leptonic pion decays, most theories require that leptoquarks be essentially chiral. Another such restriction on leptoquarks is regarding their couplings or lepton-quark quantum numbers. There is no a priori reason that leptoquarks cannot couple to any lepton-quark combination. However the obvious downfall of a leptoquark theory with universal couplings would be that such leptoquarks would give rise to flavor-mixing-induced FCNC. It is important to note that although one can require leptoquarks to have chiral and family diagonal couplings, the $S U(2)_{L}$ eigenstates of the quarks and leptons are not their mass eigenstates and thus lepton-quark mixing can still give rise to inter-generation decays so long as the charge is conserved [67]. Examples of such flavor-mixing-induced FCNC would be $K_{L}^{0} \rightarrow e \mu, D^{0} \rightarrow \pi^{0} e \mu$, or $B^{0} \rightarrow \mu \tau$ 


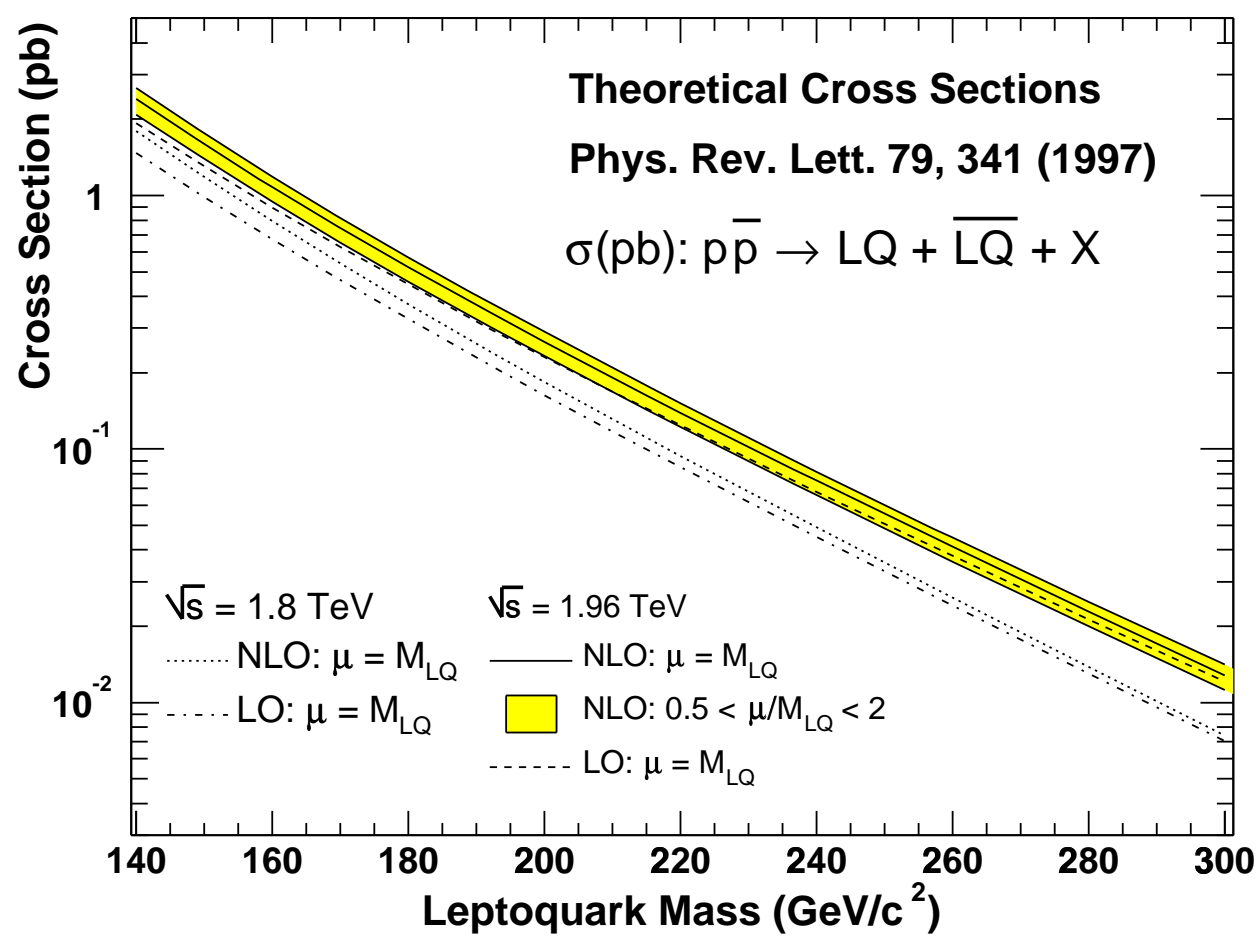

Figure 2.9: The theoretical scalar leptoquark cross section as a function of leptoquark mass at the Run I and Run II Tevatron.

as shown in Figure 2.10. However, the bounds that are obtainable from such FCNC involve several unknown parameters and are weaker than experimental limits set by direct searches.

Experimental limits on FCNC indicate that flavor-mixing is severely suppressed and thus leptoquarks are assumed to decay uniquely within a lepton-quark generation. In this analysis we focus on leptoquarks exhibiting family-diagonal decays. Figure 2.11 shows two possible decays for a second-generation leptoquark. The parameter $\beta$ is the branching ratio of leptoquark decays into a charged lepton-quark pair and a neutrino-quark pair. Since we search for leptoquarks in pairs, there is a combinatorial ambiguity as to which leptoquark decays into a charged lepton and quark. The combinatoric rates are given by:

$$
\begin{aligned}
L Q_{2} & \rightarrow q_{2} \mu^{ \pm} & B R & =\beta \\
L Q_{2} & \rightarrow q_{2} \nu_{\mu} & B R & =1-\beta .
\end{aligned}
$$

Therefore, the combinatoric rates for the channels $\ell \ell q q, \ell \nu q q$ and $\nu \nu q q$ channels are respectively $B R=\beta^{2}, 2 \beta(1-\beta)$, and $(1-\beta)^{2}$. 


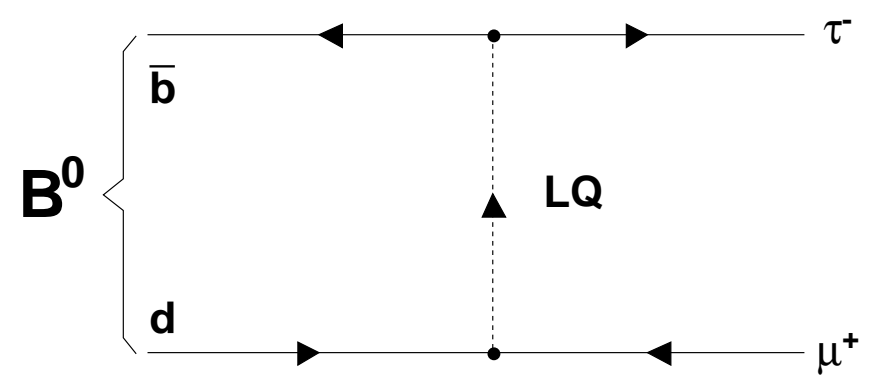

Figure 2.10: An example of a leptoquark induced FCNC decay process $B^{0} \rightarrow \mu \tau$. The leptoquark $L Q$ couples with more than one lepton-quark generation.

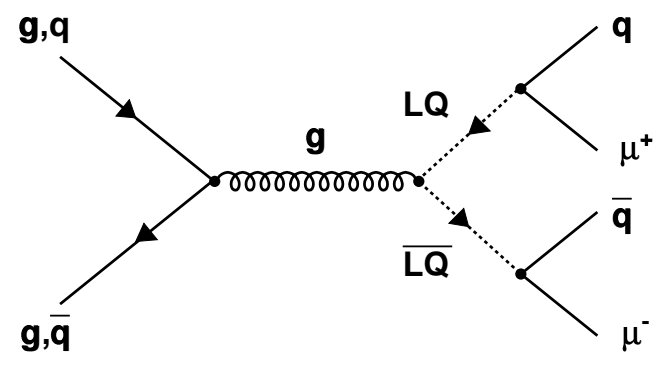

$\beta=1$

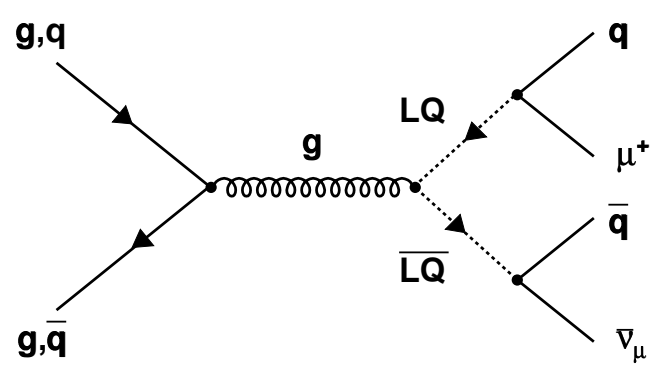

$\beta=1 / 2$

Figure 2.11: Second generation scalar leptoquark decay for $\beta=1$ (left) and $\beta=1 / 2$ (right).

\subsection{Existing Leptoquark Mass Limits}

There have been a great number of papers published on experimental leptoquark mass limits within the last decade due to a hint of a leptoquark signature seen at HERA. In February, 1997, the H1[4] and ZEUS[105] collaborations working on $e^{+} p$ collisions at HERA simultaneously released papers reporting an excess of NC events with high $Q^{2}$. H1 found 12 events with $Q^{2}>15,000 \mathrm{GeV}$ where the SM predicts $4.71 \pm 0.76$, and ZEUS found 2 events with $Q^{2}>35,000 \mathrm{GeV} / \mathrm{c}^{2}$ in comparison to the $\mathrm{SM}$ prediction of $0.145 \pm 0.013$ events for that range of $Q^{2}$. The probability that these excesses were due to statistical fluctuation was $0.5 \%$. Then at the Lepton-Photon conference in 1997, H1 and ZEUS reported more NC events as well as CC events from data taken up to June of that year [21][65]. Collectively, they found $22 \mathrm{CC}$ events with a $Q^{2}>10,000 \mathrm{GeV}$ where the SM predicts $17.7 \pm 4.3$ events. So, any leptoquark theory would have to account for not only neutral currents, but charged currents as well. 
What made the excess more interesting was that $\mathrm{H} 1$ observed a cluster of the events at a mass of about $200 \mathrm{GeV} / \mathrm{c}^{2}$. This excess could be explained by the existence of first generation scalar leptoquarks, including scalar squarks in supersymmetric theories with R-parity breaking. But if low-mass scalar leptoquarks exist, they would be produced at significant rates at the $p \bar{p}$ Tevatron Collider. So immediately both CDF [54] and DØ[69] conducted searches for leptoquarks using Run I Tevatron data and reported a combined lower limit of $M_{S}>242 \mathrm{GeV} / \mathrm{c}^{2}$ [36]. For the vector leptoquark case, even at choices of $\kappa$ and $\lambda$ such that the cross section is minimized [31], the lower mass bounds were significantly higher than the scalar limit.

The leptoquark searches from Run I at the Tevatron exclude the interpretation of the excess events found at HERA as being a result of the production of a leptoquark state with chiral and family-diagonal couplings to fermions [78]. ZEUS also observed a cluster in their data, but at a mass of about $220 \mathrm{GeV} / \mathrm{c}^{2}$. This difference in energy between $\mathrm{H} 1$ cluster and ZEUS's cluster was later ruled out by being the cause of initial state radiation or detector effects [21]. Thus it has been ruled unlikely that the excess observed at HERA was due to the production and decay of a single narrow resonance.

Since then, the data collected at H1 and ZEUS has doubled, and no excess events have been observed. For $e p$ collisions, the production cross section does depend on the leptoquark coupling and thus mass limits have been set by both collaborations which depend on $\lambda_{\ell q}$. Figure 2.12 shows the leptoquark mass limits as a function of $\lambda_{e u}$ for the $\beta=1$ case. The diagram also shows the limits set by LEP. Figure 2.12 shows how the Tevatron limits relate to the mass limits set by LEP and HERA. For large coupling value, LEP and HERA limits are very high. The Tevatron limits are able to probe mass regions having a small coupling constant. The published Run I Tevatron results are listed in Table 2.3. Preliminary results of scalar leptoquark searches at the both the Run I and Run II Tevatron are shown in Table 2.4 . 


\begin{tabular}{|c|c|c|c|}
\hline Channel & $\mathcal{L}, \mathrm{pb}^{-1}$ & $M, \mathrm{GeV} / \mathrm{c}^{2}$ & Comments \\
\hline \multicolumn{4}{|c|}{ First Generation } \\
\hline$e e q q$ & 110 & 213 & CDF Collaboration [37] \\
\hline$e e q q$ & 115 & 225 & combined result, DØ Collaboration [46] \\
\hline$e e q q$ & 123 & 225 & DØ Collaboration [47] \\
\hline$e e q q$ & 233 & 242 & $\mathrm{CDF}$ and $\mathrm{D} \varnothing$ combined result \\
\hline$e \nu q q$ & 115 & 204 & combined result, $\mathrm{D} \varnothing$ Collaboration [46] \\
\hline$\nu \nu q q$ & 85.2 & 98 & $\mathrm{D} \varnothing$ Collaboration $[51]$ \\
\hline \multicolumn{4}{|c|}{ Second Generation } \\
\hline$\mu \mu q q$ & 110 & 202 & CDF Collaboration [38] \\
\hline$\mu \mu q q$ & 94 & 200 & combined result, $\mathrm{D} \varnothing[48]$ \\
\hline$\mu \nu q q$ & 94 & 160 & $\mathrm{D} \varnothing$ Collaboration [49] \\
\hline$\mu \nu q q$ & 94 & 180 & combined result, DØ Collaboration [48] \\
\hline$\nu \nu c c$ & 88 & 123 & CDF Collaboration [41] \\
\hline \multicolumn{4}{|c|}{ Third Generation } \\
\hline$\tau \tau b b$ & $60,20,92$ & 94 & DØ Collaboration [50] \\
\hline$\tau \tau b b$ & 110 & 99 & CDF Collaboration [39] \\
\hline$\nu \nu b b$ & 88 & 148 & CDF Collaboration [41] \\
\hline
\end{tabular}

Table 2.3: Published results of scalar leptoquark searches from the Run I Tevatron. $\mathcal{L}$ is the time-integrated luminosity and $M$ is the leptoquark mass below which scalar leptoquarks have been excluded at the $95 \%$ C.L. The $\tau \tau b b$ channel was analyzed by D $\varnothing$ using three sets of data collected using different triggers.

\begin{tabular}{lccl}
\hline Channel & $\mathscr{L}, \mathrm{pb}^{-1}$ & $M, \mathrm{GeV} / \mathrm{c}^{2}$ & Comments \\
\hline \multicolumn{2}{l}{ First Generation } & & \\
eeqq & 175 & 238 & D $~$ \\
$e e q q$ & 203 & 230 & CDF Run II Preliminary \\
$e \nu q q$ & 175 & 194 & D $\varnothing$ Run II Preliminary \\
$e \nu q q$ & 72 & 166 & CDF Run II Preliminary \\
$\nu \nu q q$ & 191 & $78-117$ & CDF Run II Preliminary [24] \\
Second Generation & & \\
$\mu \mu q q$ & 104 & 186 & DØ Run II Preliminary \\
$\mu \mu q q$ & 109 & 209 & CDF Run I Preliminary, combined result [82] \\
$\mu \mu q q$ & 126 & 206 & CDF Run II Preliminary [87] \\
$\mu \nu q q$ & 109 & 164 & CDF Run I Preliminary [83] \\
$\mu \nu q q$ & 109 & 183 & CDF Run I Preliminary, combined result [82] \\
\hline
\end{tabular}

Table 2.4: Preliminary results of scalar leptoquark searches at the Run I and Run II Tevatron. 


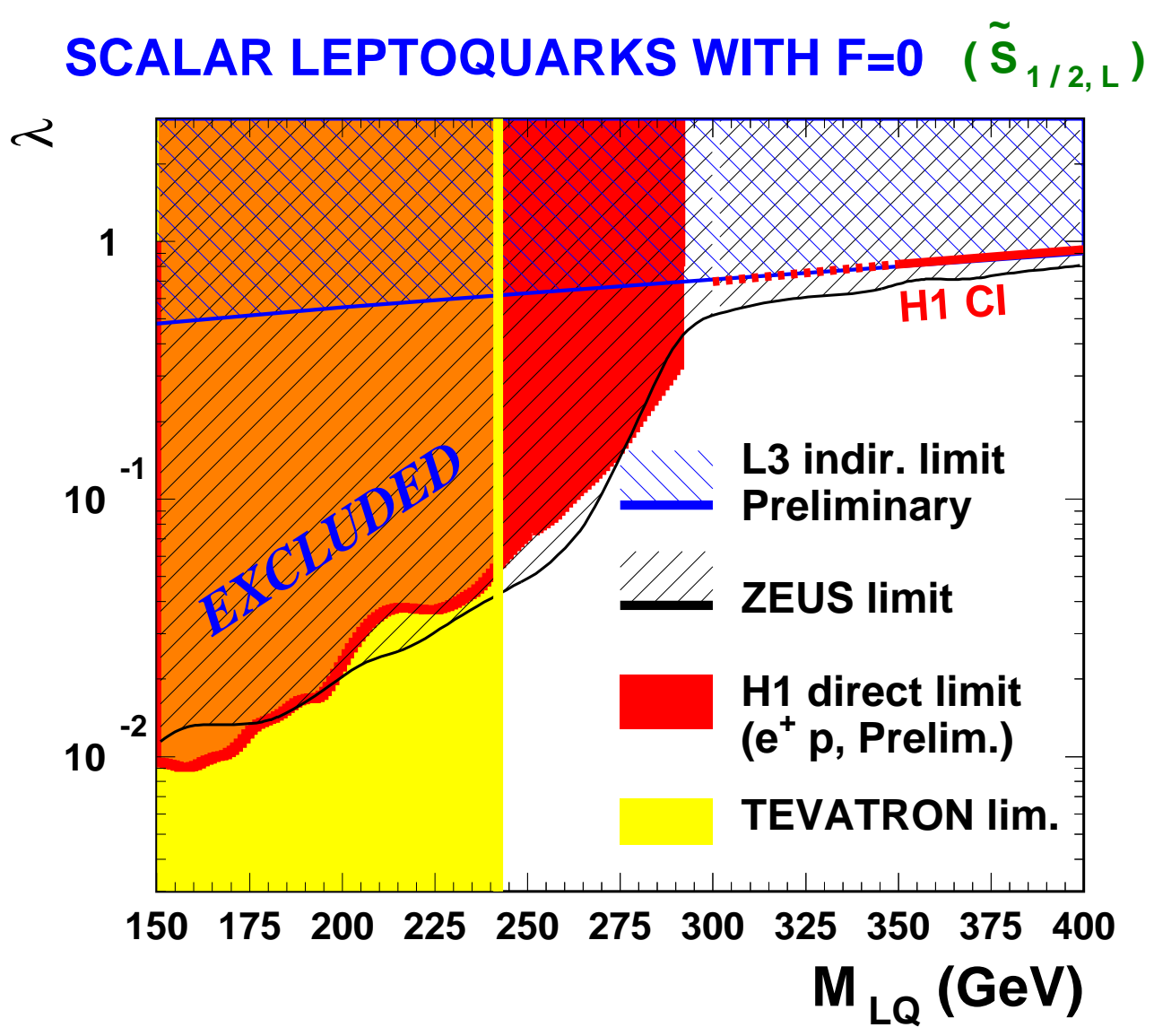

Figure 2.12: Limits on the first-generation scalar leptoquark mass as a function of the leptoquark coupling (from ZEUS Collaboration [104]). The limit for the Tevatron, which is independent of the leptoquark coupling, is from combined CDF and D $\varnothing$ results from Run I. 


\section{Part II}

\section{Experimental Apparatus}




\section{Chapter 3}

\section{The Apparatus}

This chapter presents the experimental apparatus used to create and detect the particle interactions in which leptoquark signatures are sought. Both the accelerator chain and collider detector have undergone significant upgrades since the Run I period of 1992 to 1995. First the chain of particle accelerators used to generate the p $\bar{p}$ collisions is outlined. Then the detectors used in recording the kinematics of the particle collisions are briefly described. Emphasis is given to detector components which are important for the leptoquark signatures examined in this dissertation. Finally the hardware system used in filtering the physics events and recording them to tape is outlined.

\subsection{Run II Proton Producton and Acceleration}

In order to discover new physical phenomena such as leptoquarks, it is necessary both to generate sufficient energy and statistics in order to produce the new phenomena and to probe and measure the phenomenon of interest. The energy is needed to probe the constituents and interactions of matter; the statistics are needed to do so with a high level of precision. To this end, the Fermi National Accelerator Laboratory (Fermilab), located on 6,800 acres of land approximately 40 miles west of Chicago, IL, has built a series of particle accelerators which collectively focus and propel protons and anti-protons to relativistic velocities.

Fermilab has two modes of running: $p \bar{p}$ collisions and fixed target. In fixed target mode, proton beams are delivered down extraction beamlines and aimed at stationary targets. In collider mode, both protons and anti-proton beams are accelerated and kept separate until brought together at specific interaction points surrounded by large particle detectors where the resulting products of their collisions are detected and recorded.

The advent of particle colliders is fairly recent in the history of high energy physics. The first operational particle colliders were electron-positron colliders. These were soon followed 


\begin{tabular}{|lccll|}
\hline Machine & $E$-Injection & $E$-Extraction & Beam & Destination \\
\hline \hline Preacc & rest & $750 \mathrm{KeV}$ & $p$ & Linac \\
\hline Linac & $750 \mathrm{KeV}$ & $400 \mathrm{MeV}$ & $p$ & Booster \\
\hline Debuncher & $400 \mathrm{MeV}$ & $8 \mathrm{GeV}$ & $\bar{p}$ & \\
\hline Accumulator & $8 \mathrm{GeV}$ & $8 \mathrm{GeV}$ & $\bar{p}$ & \\
\hline Booster & $8 \mathrm{GeV}$ & $8 \mathrm{GeV}$ & $p$ & Main Injector \\
\hline Main Injector & $8 \mathrm{GeV}$ & $120 \mathrm{GeV}$ & $p, \bar{p}$ & Switchyard, Accumulator \\
& $8 \mathrm{GeV}$ & $150 \mathrm{GeV}$ & $p, \bar{p}$ & Tevatron \\
\hline Tevatron & $150 \mathrm{GeV}$ & $960 \mathrm{GeV}$ & $p, \bar{p}$ & Collision \\
\hline
\end{tabular}

Table 3.1: The components of the accelerator chain at Fermilab. The energy and destination of the beam depends on the operational mode of the accelerator.

by the construction of a proton-antiproton collider at CERN in the early 1980's called the SPS. The advantage of colliding particles with their antiparticles is that the center of mass energy is twice that of a fixed target experiment. The SPS obtained a center of mass energy of $\sqrt{s}=630 \mathrm{GeV}$ with each beam at $315 \mathrm{GeV}$. At Fermilab, a synchrotron accelerator called the Tevatron was originally commisioned as an $800 \mathrm{GeV}$ accelerator for fixed target experiments. In 1986 an anti-proton source was comissioned and the first $p \bar{p}$ collisions took place that year, marking the beginning of Run I.

In Run I, Fermilab collided proton and anti-proton beams with a total center-of-mass energy of $\sqrt{s}=1.8 \mathrm{TeV}$. In Run II, several components of the chain of accelerators used to achieve this energy were modified or replaced to increase the center-of-mass energy to $\sqrt{s}=1.96 \mathrm{TeV}$. The most significant change to the chain of accelerators was the replacement of the Main Ring with the Main Injector in 1998. The various components of the accelerator chain and their associated energies are listed in Table 3.1.

The locations of these accelerators and storage rings are shown in Figure 3.1. The final beam configuration for Run II is 36 proton bunches traveling clockwise and 36 antiproton bunches traveling counter-clockwise in the Tevatron ring. The following sections outline the steps and machinery used in obtaining the final beam configuration. First, the proton preparation stages are briefly described followed by a description of the antiproton preparation.

\subsubsection{Proton Production and Acceleration}

The first stage of proton acceleration begins with the creation of hydrogen ions, $H^{-}$. These ions are created by first placing hydrogen atoms in an electric field where they are stripped of their electrons. The positive hydrogen ions, $H^{+}$, are attracted to a plate of cesium whose outer electrons have a low work function. Periodically, an incoming proton 


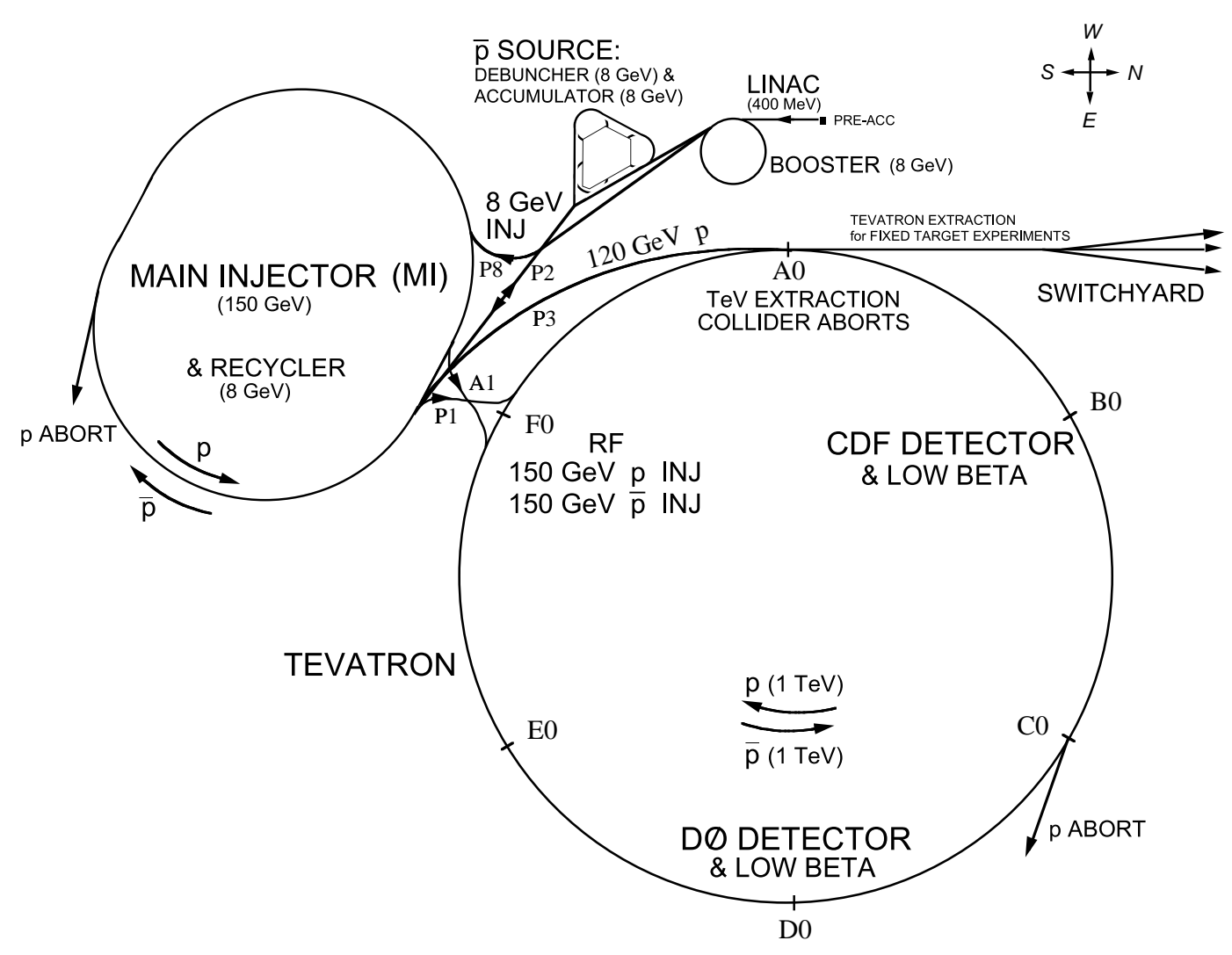

Figure 3.1: An aerial diagram of the Run II accelerator chain. Although the Run II Tevatron was designed to run at $1 \mathrm{TeV}$, the Run II nominal energy is $960 \mathrm{GeV}$. It is divided into 6 sectors divided by straight sections labeled A0 through F0. The CDF II is located at B0. [23]

will dislodge from the cesium surface another proton with two attached electrons to create a negatively charged hydrogen ion. In the presence of the electric field the negative hydrogen ions will move in the direction away from the cesium plate.

The $H^{-}$ions are collected and accelerated by a $750 \mathrm{KV}$ DC voltage source called a Cockcroft-Walton electrostatic generator. The $750 \mathrm{KV} H^{-}$ions exit the generator and enter a 130m long Alvarez type linear accelerator called the Linac where their energy is increased to $400 \mathrm{MeV}$. The linac uses radio frequency ( $\mathrm{RF}$ ) power to create a time-varying electric field. The $H^{-}$ions are shielded from the electric field such that they only see the electric field in one direction. Since the ions are accelerating, so too must the shielding and gaps increase in length in the RF cavities. The Linac is capable of peak performance of accelerating one batch of protons at a rate of $15 \mathrm{~Hz}$, or once every $66 \mathrm{~ms}$, though a beam is rarely demanded that frequently. Because the beam is accelerated using RF cavities, each cycle worth of accelerated beam, or "batch", is not a continuous stream of protons but is 
broken up into "bunches". These bunches are protons congregated about a particular phase of the RF wave. The number of bunches in a batch is determined by the length of the accelerator and the length of the RF wave.

The Linac has two modes of operation. A Neutron Therapy Facility located in the Linac gallery uses the beam to strike a target. In this mode the ions are accelerated only to 66 $\mathrm{MeV}$. The resulting secondary neutrons are directed towards cancerous tumors. In high energy mode, the $400 \mathrm{MeV}$ ions are either dumped to a large concrete block or pass through a thin carbon foil which strips them of their electrons leaving only the protons to enter the Booster.

The Booster is a rapid cycling synchrotron which accelerates the protons from $400 \mathrm{MeV}$ to $8 \mathrm{GeV}$ at a rate of $15 \mathrm{~Hz}$, or in a period of $66 \mathrm{~ms}$. It is comprised of $18 \mathrm{RF}$ cavities and has a circumference of $475 \mathrm{~m}$ which means the protons travel about 16,000 laps during this time. Magnets positioned around the synchrotron act to keep the beam focused and to keep their orbits circular. At most, there can be 48 proton bunches in a Booster batch. Batches of protons are repeatedly accelerated and extracted in order to fill up the next accelerator ring called the Main Injector.

The Fermilab Main Injector (FMI) is a synchrotron accelerator in which the energy of the $8 \mathrm{GeV}$ proton bunches is increased to $150 \mathrm{GeV}$ [45]. In 1998, the FMI replaced the Main Ring of Run I which was located directly above the Tevatron. The ring's circumference is 28/53 times that of the Tevatron ring and 7 times that of the Booster. The FMI design goals were to lower the cycle time to $1.5 \mathrm{sec}$ and to improve the Main Ring acceleration rate of $120 \mathrm{GeV} / \mathrm{s}$ to $240 \mathrm{GeV} / \mathrm{s}$.

The FMI has several modes of operation and is capable of accelerating both protons and antiprotons. It can operate simultaneously in fixed target and antiproton production modes, in which the beam is only brought to $120 \mathrm{GeV}$. More details on these modes are discussed in the next section. For preparation of protons into the Tevatron, only 7 of the 84 bunches per Booster batch are extracted into the FMI while the remaining 77 are sent to the Booster dump. These 7 bunches are then coalesced to form one narrow, high intensity bunch. A 1.45 second "flattop" is required for the bunch coalescing. The $150 \mathrm{GeV}$ high intensity bunch is then injected into the Tevatron. These steps are repeated until there are a total of 36 proton bunches in the Tevatron.

\subsubsection{Antiproton Production and Acceleration}

The accumulation of antiprotons is a far more challenging process than for the protons, and is the largest bottleneck in the proton-antiproton preparation. This is largely due to the inherent inefficiency of producing the antiprotons. It takes about $10^{5}$ protons striking a target to produce just one or two antiprotons and thus hours are needed to accumulate a 
suitable amount of antiprotons to use in a Tevatron collider store.

The antiproton "source" is actually comprised of a target station and two rings called the Debuncher and the Accumulator. Antiproton production begins by loading a Booster batch of protons into the Main Injector and accelerating the batch to $120 \mathrm{GeV}$. The protons are then extracted and the beam is directed and focused on a $7 \mathrm{~cm}$ thick nickel target. The resulting secondary particles shower off the target but are focused using a lithium lens. The particles are then filtered using a pulsed magnet which acts as a charge-mass spectrometer and selects the antiprotons which have an energy of approximately $8 \mathrm{GeV}$. This process happens about every 1.5 seconds. For every 1 million protons that hit the target, only about twenty $8 \mathrm{GeV}$ antiprotons survive.

The collected antiproton bunches are sent to the Debuncher, an $8 \mathrm{GeV}$ rounded, triangular shaped synchrotron which both evens the energy spread (but widens the time spread) and stochastically cools the antiprotons. The antiprotons are bent around the three corners of the triangle so that the more energetic, and hence more massive, antiprotons will lag behind the less energetic antiprotons. It takes approximately $100 \mathrm{~ms}$ to debunch which is a much smaller period of time in comparison to the Main Injector frequency of only $1.5 \mathrm{~Hz}$. The extra time is used to cool the antiprotons via stochastic cooling. Information on the energy spread from one end of the beam is used by RF magnets which produce an EM field on the other end of the beam. The RF phase slows down and evens out the energy distribution while broadening the spatial distribution. The debunched antiprotons are then sent to the Accumulator, an $8 \mathrm{GeV}$ synchroton located on the inside perimeter of the debuncher which accumulates and stores, or "stacks", the antiprotons. The antiprotons remain in the accumulator until a sufficient number have been stacked.

The Tevatron is the final destination of the protons and antiprotons before collision. First, $150 \mathrm{GeV}$ proton bunches are loaded from the Main Injector and into the Tevatron in 3 groups of 12 evenly-spaced bunches. Next, the antiprotons are sent from the Accumulator back into the Main Injector where they are accelerated from $8 \mathrm{GeV}$ to $150 \mathrm{GeV}$. The antiproton bunches are loaded one bunch at a time into the Tevatron to make another 3 groups of 12 evenly spaced bunches. The Tevatron Ring then accelerates the bunches to an energy of $980 \mathrm{GeV}$.

The Tevatron ring features 1000 cyrogenically cooled super conducting magnets and is approximately $6 \mathrm{~km}$ in circumference. The ring is divided into six sectors labeled A through F. Each sector has straight sections which are serviced by buildings labeled " 0 ". Their locations are shown in Figure 3.1. The proton and antiproton bunches are allowed to cross paths at the B0 and D0 points where particle detectors are positioned. The bunches are spaced such that there are 36 bunches crossings every 396 ns. This crossing time impacts the design of the electronic triggering system, which is discussed in Section 3.3. The Tevatron 
ring essentially stores the proton and antiproton beams while they are allowed to collide at the D0 and B0 points. The store ends when another batch of antiprotons is ready or the beam is aborted due to malfunction.

\subsection{The Run II Collider Detector at Fermilab}

The Run II Collider Detector at Fermilab (CDF II) is a conglomerate of individual particle detection systems optimally designed and positioned to track the paths and measure the energies of particles. The detector is located along the B0 straight section of the Tevatron ring. A cross section of the detector is illustrated in Figure 3.2. The detector is solenoidal in shape and is both azimuthally symmetric and forward/backward symmetric along the beamline and is centered at the nominal beam crossing point.

The placement of the subdetectors and the overall geometry of the detector reflects the manner in which particles interact with matter. The detector volume contains enough material to stop all of the particles created from the $p \bar{p}$ collisions. Tracking systems are located within a superconducting solenoid capable of generating a 1.4 Telsa field parallel to the beam axis which helps distinguish charged particles. Closest to the interaction point are the silicon-vertex detectors capable of providing stand-alone silicon segments. The calorimetry, which has fine graining for locatiang and measuring electromagnetic and hadronic showers, is situated just outside of the solenoid. Finally, the muon drift chambers, insulated with layers of steel, are located at the outermost region of the detector.

The CDF II is situated along the Tevatron beam line such that the interaction point, which serves as the coordinate system origin, is at the center of the detector. The positive $z$-axis is defined to be collinear with the beam-line and pointing along the direction of the proton beam (EAST). The $y$-axis points directly up and the $x$-axis points outward (NORTH) from the center of the detector ring. At hadronic colliders in which the incident particles have relativistic velocities, it is useful to use the "rapidity" variable to define their outgoing angle [85]. Therefore, given the high-energy of the particles and the cylindrical geometry of the detector, it is most useful to use a coordinate system at the CDF II represented by: $r$, the distance from the detector center; $\phi$ the angle with $x$ in the $x-y$ plane; and $\eta$, or "pseudorapidity", a massless approximation of the rapidity defined in terms of $\theta$ :

$$
\eta=-\log \tan \left(\frac{\theta}{2}\right)
$$

where $\theta$ is the azimuthal angle in the $z-y$ plane. The pseudorapidity is equal to zero at $\theta=0$ and is positive in the direction of the protons. In this thesis, we define the "central" region of the detector by $|\eta|<1.0$ and the "forward" region by $|\eta|>1.0$. 


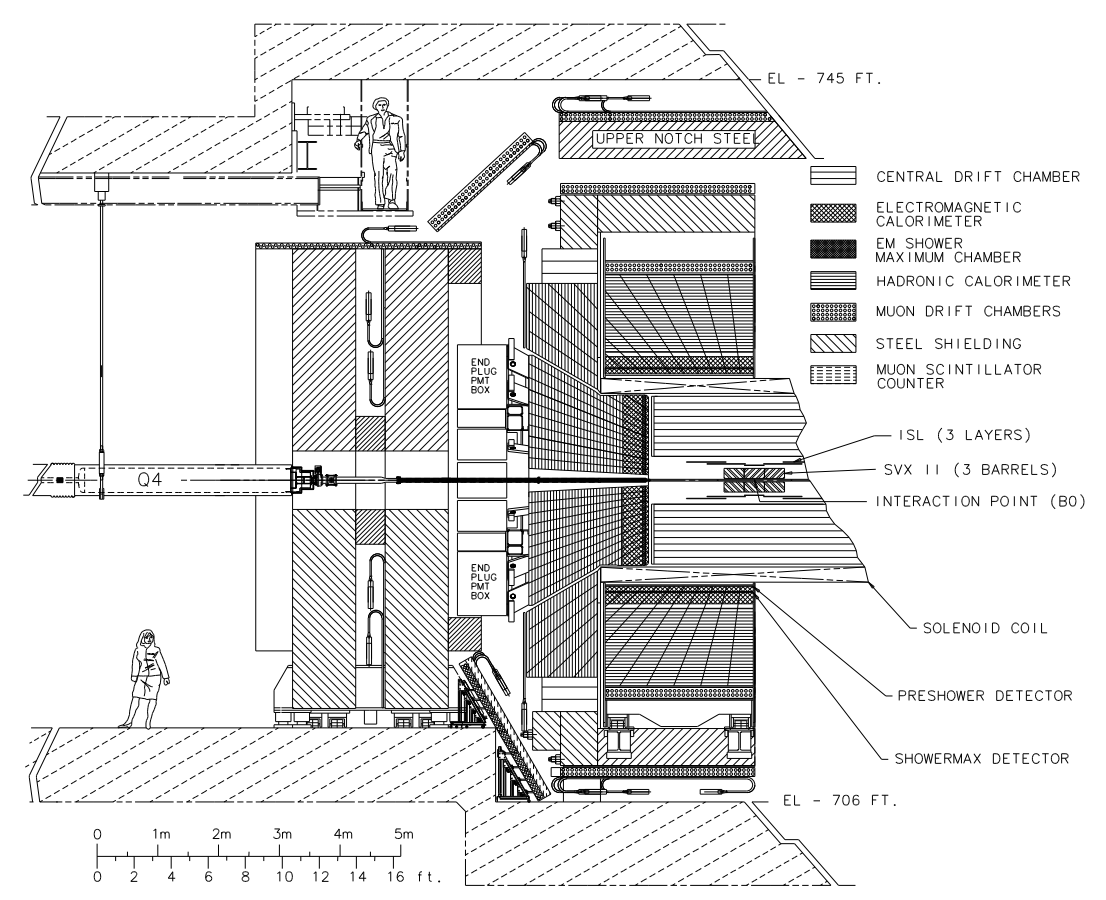

Figure 3.2: A cross section schematic of the CDF II detector [42].

Related to the CDF II $\eta, \phi$ coordinate system is the geometric quantity of "cone size", defined as $\Delta R=\sqrt{\Delta \eta^{2}+\Delta \phi^{2}}$. The cone size is a geometric quantity and not a physical quantity because of the non-linear behavior of $\eta$ with respect to $\theta$. Therefore a cone size in the forward region of the detector defines a smaller physical area than in the central region. The transverse component of a particle's energy and momentum is defined by the angle of the particle's track in the $y-z$ plane with respect to the beam position, i.e. $p_{T}=p \sin \theta$ and $E_{T}=E \sin \theta$ where $p$ and $E$ are the particle's momentum and energy respectively.

The components of the CDF II have been almost entirely replaced in Run II with new hardware and triggering systems. The solenoid, central calorimeters, and CMU detectors are the only detector components remaining from Run I. With respect to Run I, the Run II upgrades accommodate higher luminosities via a finer detector granularity to help maintain low occupancy levels, and shorter bunch-crossing times via faster readout and triggering electronics. The following sections describe in detail some of the specifics of the subdetectors of interest used in second-generation leptoquark searches. Original design and construction details can be found in [42]. 


\subsubsection{Tracking}

The CDF II tracking system is capable of reconstructing 3-dimensional paths of charged particles. The entire tracking system is enclosed by a solenoid making it possible to measure the momentum of charged particles passing through the tracking region. The detector components of the tracking system consist essentially of three parts: an inner tracker consisting of both the Run II Silicon Vertex Detector (SVXII) and the Inner Super Layer (ISL), and an outer tracker called the Central Open-cell Tracker (COT). Figure 3.3 shows one quadrant of the tracking volume in the $r-z$ plane.

\section{CDF Tracking Volume}

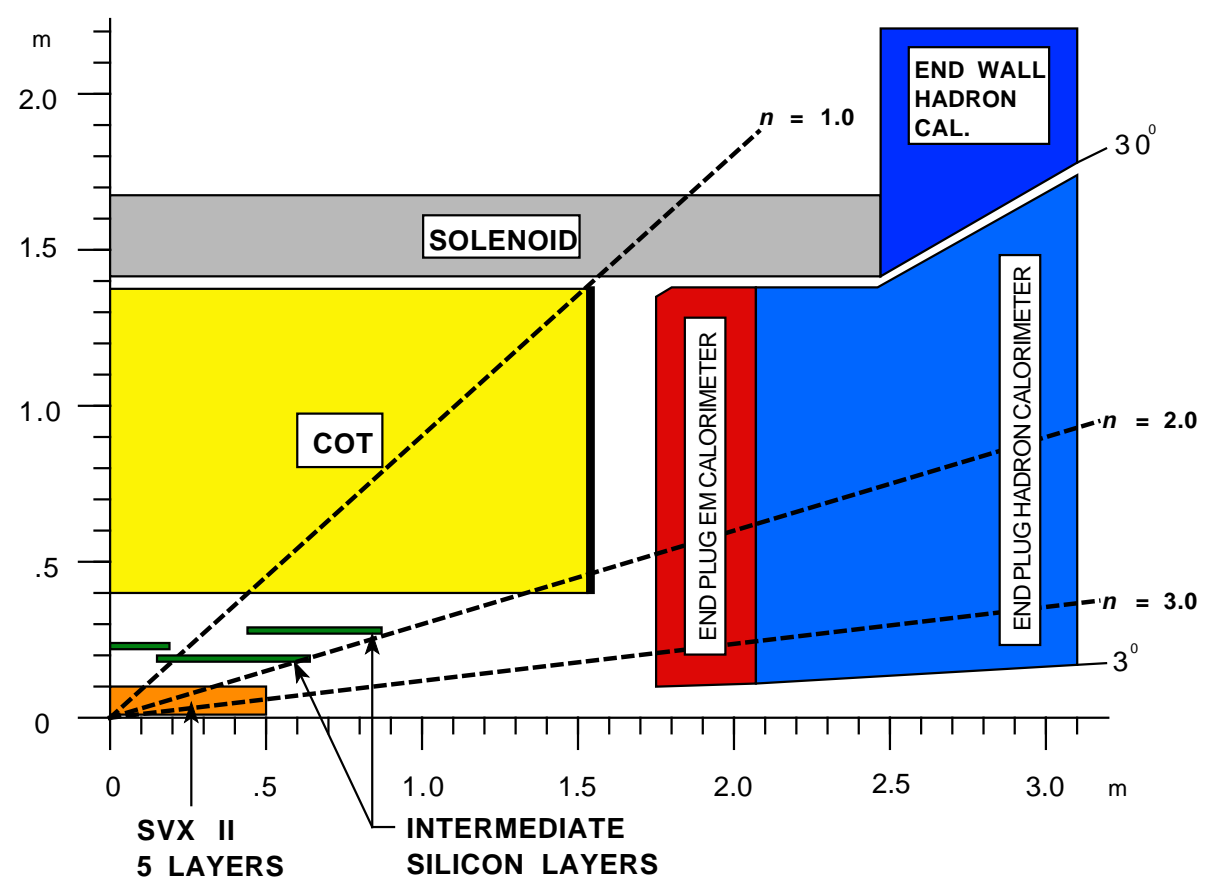

Figure 3.3: A cross section of one quadrant in the $r-z$ plane of the CDF II tracking volume [42].

The SVXII [40] consists of 5 layers of a combination of 90-degree and small-angle $\left(1.2^{\circ}\right)$ stereo layers of double-sided silicon sensors. The entire SVX detector is composed of 3 barrels covering a total $z$-distance of $96.0 \mathrm{~cm}$, or $|\eta| \leq 2.0$, which is roughly twice as long as the SVX detector in Run I. The 5 silicon layers cover a radial distance of 2.4 to $10.7 \mathrm{~cm}$ from the beam line. The SVX detector is capable of a resolution of $12 \mu \mathrm{m}$. In order to suppress the creation of secondary particles and reduce multiple scattering, the amount of material used to construct the SVXII was kept to a minimum, approximately 3.5 radiation lengths $\left(\mathrm{X}_{0}\right)$. In all there are a total of 405,504 channels in the system which are connected 
to readout chips which have been radiation hardened. Due to the length of the barrels, the readout speed and capacitance limitations, the readout chips are located close to the silicon sensors and within the active volume of the detector. The consequence is a reduction in resolution of the $r \phi$ impact parameter. To compensate for this effect, an additional singlesided silicon layer called Layer00 is mounted on the beam-pipe. As of this writing L00 has not yet been incorporated into the online or offline tracking system.

The ISL consists of three separate silicon layers extending in overlapping regions of $\eta$. A single ISL layer covering $|\eta|<1$ is positioned $22 \mathrm{~cm}$ from the beam line. Two more layers are positioned at a radii 20 and $28 \mathrm{~cm}$ and cover the region $1.0 \leq|\eta| \leq 2.0$. The readout electronics are identical to the SVX. Collectively, the ISL and SVX are capable of providing standalone tracking in the region $|\eta| \leq 2.0$.

The COT is an open-cell drift chamber capable of detecting charged particles [28] similar to the Central Tracker Chamber (CTC) of Run I. It encloses the SVX and ISL by extending radially from 44 to $132 \mathrm{~cm}$ and has an effective pseudorapidity tracking region of $|\eta| \leq 1.0$. Each drift cell comprises 12 alternating sense and shaper wires running down the middle of two gold-on-mylar cathode planes separated by about $2 \mathrm{~cm}$ (Figure 3.4) [42]. The ends of the wires are strung between two precision-milled endplates giving a wire tension of 135 g. There are a total of 96 layers of drift cells divided into 8 "superlayers" which alternate between 4 axial and 4 stereo superlayers. The axial superlayers run parallel to the beam line whereas the stereo superlayers alternate in tilt by $\pm 3^{\circ}$ with respect to the beam line. The stereo superlayer geometry makes track reconstruction in the $r-z$ plane possible. Due to the solenoid's large magnetic field, the drift cells are oriented at a $35^{\circ}$ degree angle in the $r-\phi$ plane (Figure 3.5) to accommodate drift trajectories in the $\phi$-direction and to optimize for track measurements transverse to the beam line. A gas with a higher drift velocity and smaller drift cells with respect to the CTC drift cells of Run I have made the COT capable of maximum drift times of $100 \mathrm{~ns}$ and a resolution of $140 \mu \mathrm{m}$ [5].

\subsubsection{Calorimetry}

The CDF II calorimeters measure the energy deposited by the photons, jets, or electrons which have traversed the tracking volume. The calorimeters surround the solenoid in the full azimuthal range and extend in the pseudorapidity range up to $|\eta| \leq 3.0$. In addition to providing particle energy and position information, the calorimeter also plays a key role in measuring the imbalance of transverse energy in a physics event which is indicative of the presence of a neutrino.

There are two layers of calorimeters, the electromagnetic followed by the hadronic which are each segmented in $(\eta, \phi)$ into towers that project back to the $p \bar{p}$ interaction point. In both calorimeter types, multiple layers of scintillator tiles are sandwiched between sam- 


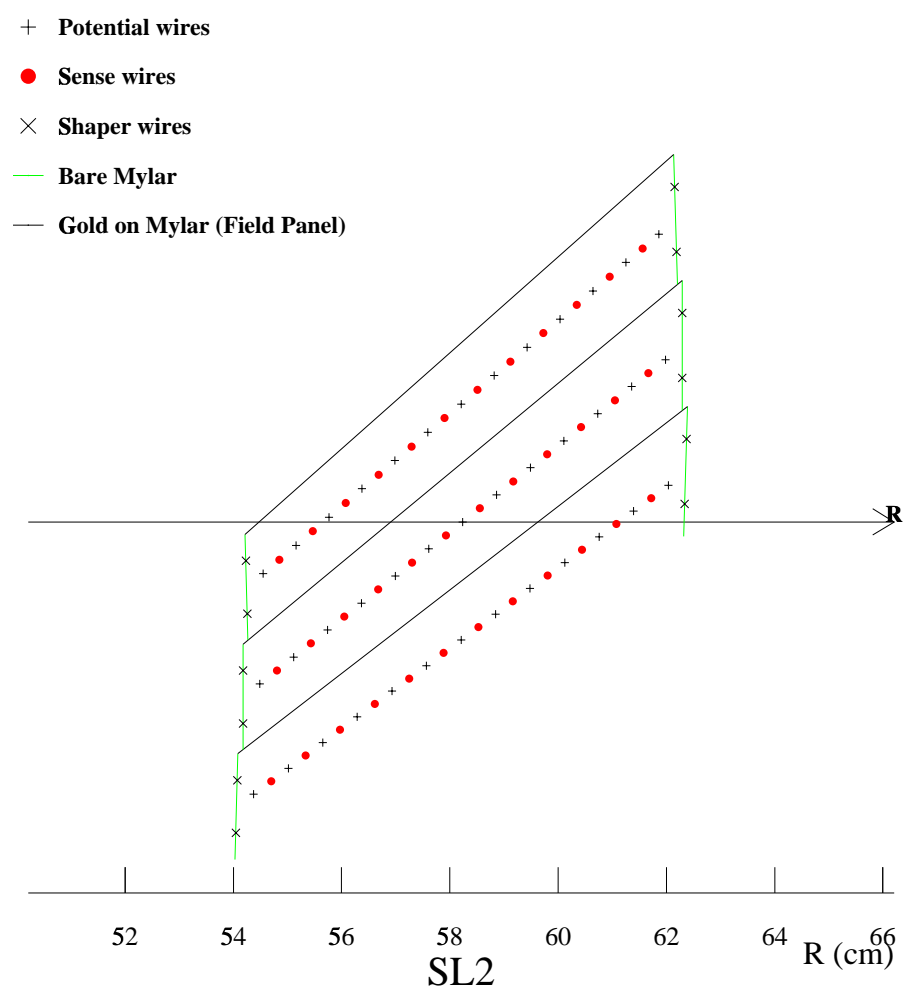

Figure 3.4: A cross section of several COT drift chambers. The wires alternate between sense wires and potential wires. The arrow shows the relative direction of the nominal beam position [42].

pling material meant to absorb the particle. The electromagnetic calorimeter uses a lead sampling material while the hadronic uses iron and in some places steel. Photons and electrons "shower" their energies in the electromagnetic calorimeter via $e^{+} e^{-}$pair production and bremmstrahlung, while hadrons deposit their energy via strong interactions in both the electromagnetic and hadronic calorimeters. The released jet energies are measured by the scintillator tiles which are embedded with wavelength shifting sheets or fibers. These fibers then carry the light to photomultiplier tubes (PMT's) located outside of the sensitive detector volume. There is no way to distinguish between jets from photons and hadrons, however electrons are distinguishable by their large ratio of electromagnetic to hadronic energy. The calorimeter gains are initially calibrated from test beam data and are retained by calibrations carried out betweeen runs via xenon flashers and LED sources.

There are a variety of subdetector calorimeter types located in the calorimeter regions of CDF II which are named the central, plug and forward regions. The acronyms for the 


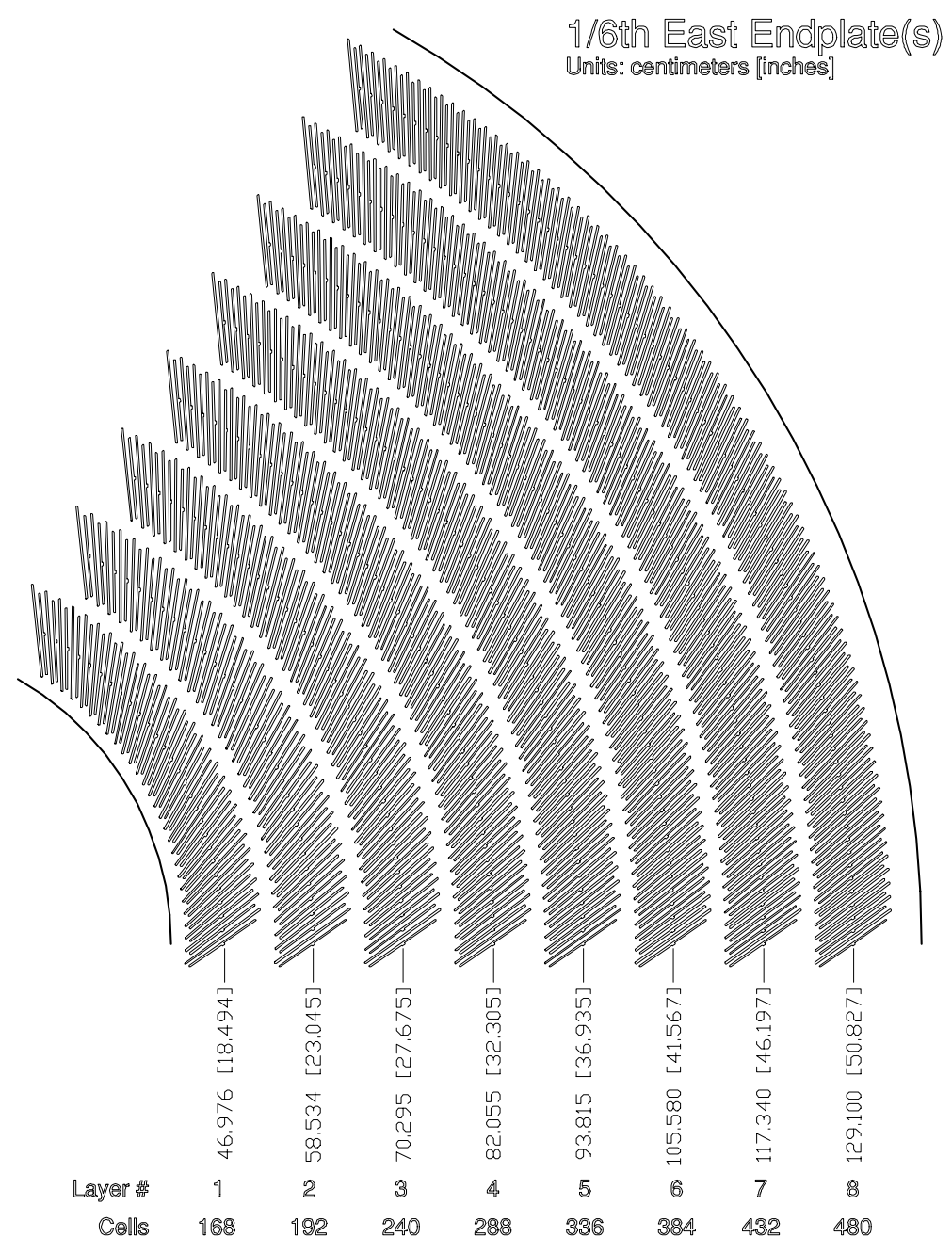

Figure 3.5: The east endplate showing the field planes of the COT cells. The numbers represent the radial distance from the beamline in centimeters[inches].

subdetectors are given in Table 3.2. The tower $(\eta, \phi)$-granularity varies from tower to tower depending on the geometric position of the tower. Table 3.3 lists the $\eta$-range and $\phi$-segmentation for the various subdetectors [30]. EM towers 10-17 and HAD towers 12-17 have a segmentation of 1 tower per $7.5^{\circ}$ opening angle in $\phi$ while the rest of the towers have an opening angle of $15^{\circ}$.

For second-generation scalar leptoquark searches, the calorimeter plays an important role in the identification of muons. Unlike electrons, muons do not undergo a significant amount of bremsstrahlung due to their heavy mass. Particles which leave behind a minimal amount of energy in the calorimeters are known as minimum ionizing particles (MIP's). The characteristic of a MIP-like particle is one criterion used in identifying muons and becomes 


\begin{tabular}{|cl|}
\hline CEM: & Central electromagnetic calorimeter \\
CHA: & Central hadronic calorimeter \\
WHA: & Wall hadronic calorimeter \\
PEM: & Plug electromagnetic calorimeter \\
PHA: & Plug hadronic calorimeter \\
CES: & Central shower maximum detector \\
PES: & Plug shower maximum detector \\
CPR: & Central preradiator detector \\
PPR: & Plug preradiator detector \\
CCR: & Central crack chamber \\
MPC: & Miniplug calorimeter \\
\hline
\end{tabular}

Table 3.2: Acronyms of the CDF II calorimeter subtector types.

\begin{tabular}{|c|c|c|c|c|c|c|}
\hline \multirow{2}{*}{$\begin{array}{c}\text { Tower } \\
\text { Type }\end{array}$} & $\Delta \eta$ & & & \multicolumn{2}{|c|}{$\phi$-granularity } & \multicolumn{2}{|c|}{ Calorimeters } \\
\cline { 4 - 7 } & & & EM & HAD & EM & HAD \\
\hline \hline 0 & $0.00-0.72$ & $0-5$ & 24 & 24 & CEM & CHA \\
\hline 1 & $0.72-0.92$ & $6-7$ & 24 & 24 & CEM & CHA,WHA \\
\hline 2 & $0.92-1.10$ & $8-9$ & 24 & 24 & CEM & WHA \\
\hline 3 & $1.10-1.20$ & 10 & $2 \times 24$ & $1 \times 24$ & PEM & WHA \\
\hline 4 & $1.20-1.32$ & 11 & $2 \times 24$ & $1 \times 24$ & PEM & WHA,PHA \\
\hline 5 & $1.32-2.11$ & $12-17$ & $2 \times 24$ & $2 \times 24$ & PEM & PHA \\
\hline 6 & $2.11-3.00$ & $18-20$ & 24 & 24 & PEM & PHA \\
\hline 7 & $3.00-3.64$ & 21 & 24 & 24 & PEM & PHA,MPC \\
\hline 8 & $3.64-5.10$ & $22-25$ & 24 & 24 & MPC & MPC \\
\hline \hline
\end{tabular}

Table 3.3: Granularity and indexing description of CDF II's eight tower types.

especially important in the case that a muon passes undetected through the muon detectors.

The calorimeters also play a crucial role in the $\beta=1 / 2$ analysis in which one leptoquark is expected to decay into a neutrino and quark. Each $p \bar{p}$ collision is assumed to begin with zero transverse momentum. This means that conservation of energy constrains the vector sum of transverse energy in a physics event to be zero. It is assumed that the product particles which traverse the tracking volume and make it to the calorimetry leave their total energy into the sensitive calorimetric material or are detected by the muon system. A neutrino, however, will pass through the detector undetected and leave behind none of its energy. If this happens then there will be an imbalance of transverse energy in the event, corresponding to the transverse energy of the undetected neutrino. At CDF II, neutrinos are reconstructed only in the sense that the missing transverse energy, $\mathbb{E}_{T}$, represents the direction and energy of the neutrino's trajectory in the $r-\phi$ plane. The calculation of the 
neutrino transverse energy is discussed further in Section 4.6.

A brief description of the CDF II calorimeter subdetectors used in this analysis follows. More specific details on these and the other subdetectors listed in Table 3.3 can be found in [42].

\section{The Central Electromagnetic Calorimeter}

The cental electromagnetic calorimeter (CEM) is comprised of $24 \times 15^{\circ}$ wedges each having 23 layers of lead $(4.5 \mathrm{~mm}) /$ scintillator $(4 \mathrm{~mm})$ material for a total thickness of $19 X_{0}$ at normal incidence. The CEM runs $4.9 \mathrm{~m}$ along the beam direction and is sectioned into 2 halves about the $\eta=0$ plane. It has a resolution of $13.5 \% / \sqrt{E \cdot \sin \theta}$ [6]. A pre-shower detector (CPR) and a shower-max detector are located respectively at the first layer and approximately $6 X_{0}$ deep into the CEM. Figure 3.6 shows a schematic of one CEM wedge.

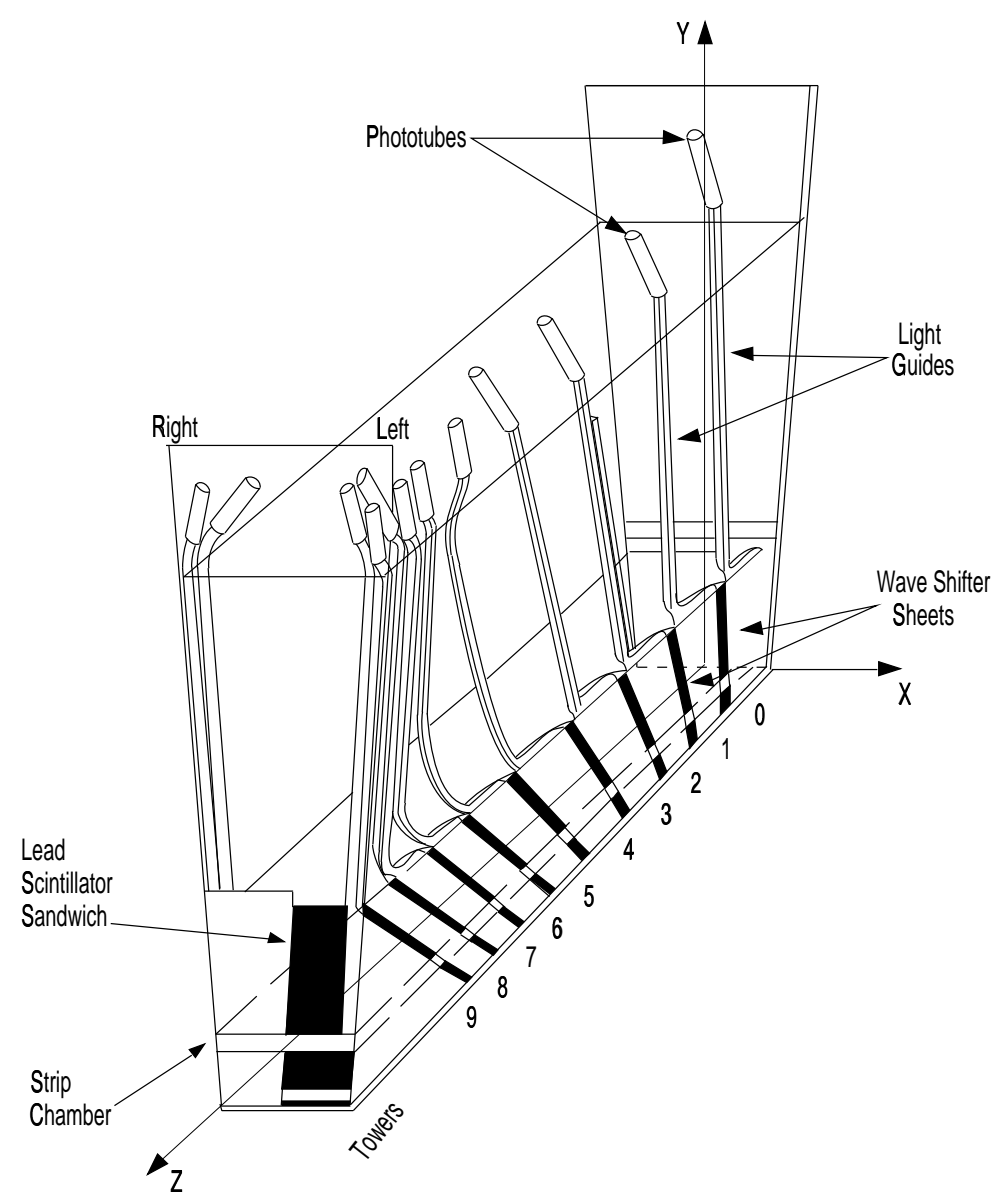

Figure 3.6: A schematic of a CEM wedge [42]. 
The CPR consists of wire chambers which use the tracking volume and solenoid as a radiator and provide information on the early development of showers. The CES consists of 2-dimensional-readout strip chambers which provide shower energy profiles and position information for matching jet showers with tracks. The transverse shower profiles are used to distinguish photons from $\pi^{0} \mathrm{~s}$ while the pulse height is used to help identify electromagnetic showers. In Run I the CES gave a factor of 2 reduction in electron fake-rates while keeping a high efficiency of true electrons [7]. In Run II this functionality has been implemented into the online level 2 trigger system which is discussed in Section 3.3 [74]. Figure 3.7 shows a schematic of the CES strip.

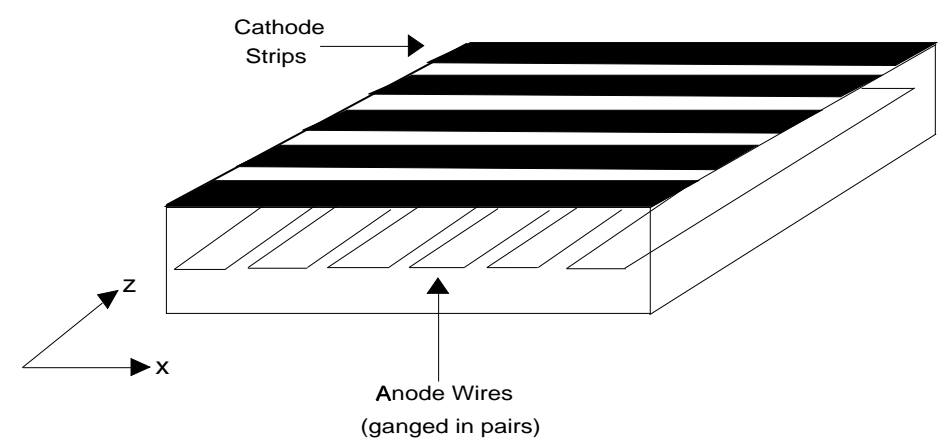

Figure 3.7: A schematic of a CES wedge [42].

The stochastic energy resolution for the CEM is determined from events having a single isolated charged meson such as a pion or a kaon. These events are known as minimum bias events. The CES has an energy resolution of $13.5 \% / \sqrt{E_{T}} \oplus 2 \%$ and a position resolution of $\pm 2 \mathrm{~mm}$ at $50 \mathrm{GeV}$ [8]. The PMT's on the backplane of the CEM are fast enough to accommodate the $132 \mathrm{~ns}$ bunches, however the CPR and CES must integrate their readouts over several bunch crossings.

\section{The Central and Wall Hadronic Calorimeter}

The central hadronic calorimeter (CHA) directly follows the central electromagnetic calorimeter which allows for convenient comparison of EM and HAD energies within a tower and straightforward implementation of calorimeter-based triggers. The CHA is comprised of 48 layers of iron $(2.5 \mathrm{~cm})$ and scintillator $(10 \mathrm{~mm})$ and has an energy resolution of $0.5 \% / \sqrt{E}$ [9]. The CHA covers the pseudorapidity region $|\eta| \leq 0.9$. The WHA extends the hadronic coverage up to $|\eta| \leq 1.3$ and has a conical shape with respect to the beam-line to accommodate the plug calorimeters. Each wedge is comprised of 48 layers of iron $(2.0 \mathrm{~cm})$ and scintillator $(10 \mathrm{~mm})$ and is mounted to the solenoid flux return. 


\section{The Plug EM and HAD}

Due to the faster bunch crossing times, the Run I gas calorimeters in the plug region $1.1<|\eta|<3.6$ have been replaced with lead/scintillator EM and iron/scintillator HAD detector components. The EM lead/scintillator wedges have 23 layers of iron(4mm) and scintillator $(4 \mathrm{~mm})$ tiles comprising a total thickness of $21 X_{0}$. The plug electromagnetic calorimeter (PEM) contains analogous features to the central electromagnetic calorimeter. A plug pre-shower detector (PPR) is located on the first EM layer and a plug showermax detector (PES) is again located at approximately $6 X_{0}$, the nominal depth of the EM shower maximum. The PPR is comprised of $10 \mathrm{~mm}$ thick scintillator tiles and the PES is comprised of two anti-aligned scintillator strips at $+22.5^{\circ}$ and $-22.5^{\circ}$ with respect to the radial dimension to provide position information in the $r-\phi$ plane. Both the PPR and PES are read out by multi-anode photomultipliers located on the back plane of each endplug. The energy resolution of the EM section is $14.4 \% / \sqrt{E} \oplus 0.7 \%$ [10].

The hadronic calorimeter wedges have 23 layers of iron $(2.5 \mathrm{~cm})$ and scintillator $(6 \mathrm{~mm})$ tiles which immediately follow the PEM calorimeter. Two stainless steel disks have been added to the back end of the PEM to increase the thickness of the hadronic calorimeter and to shield the rest of the calorimeters, including the WHA, from the solenoid's magnetic field. Stainless steel disks are also attached to the towers extending from $10^{\circ}$ down to $3^{\circ}$ from the beam line.

\subsubsection{Muon Detectors}

The muon detection systems are important in both the $\beta=1$ and the $\beta=1 / 2$ leptoquark decay channels. Because of the muon's long lifetime and penetrating ability, the muon detectors are positioned in the outermost regions of CDF II. Muons are identified by their passage through the sensitive muon detector volume which is shielded with steel absorbers to prevent passage of any surviving electromagnetic or hadronic showers. A muon's momentum is determined by its track curvature through the CDF II tracking volume. The radius of curvature for muons is very slight due to their relatively large masses. Thus, to improve muon momentum resolution, their tracks are often constrained to originate from the beamline or from a track segment in the silicon detector.

There are 4 drift chamber-based and 4 scintillator-based muon detectors collectively covering the pseudorapidity range $|\eta| \leq 2.0$. The muon systems are very similar in design and differ mainly in their positioning around the detector which is largely constrained by the geometry of the calorimeter systems and the positioning of steel absorbers. The majority of upgrades from Run I to Run II have been directed towards providing more complete

azimuthal coverage. The Run I and Run II muon system differences and upgrades are 
discussed at length in [42].

In this analysis only the central muon (CMU), central muon upgrade (CMP), and central muon extension systems (CMX) are used. These systems are compatible with the 132 ns bunch crossing times and are thus triggerable. Table 3.4 summarizes the muon detectors used in this analysis, and Figure 3.8 shows their coverage in the $\eta-\phi$ plane. There is also an intermediate muon system (IMU) covering the pseudorapidity range $|\eta| \leq 1.5$ for triggerable muons and $|\eta| \leq 2.0$ for offline muons. This system has not yet been functionally integrated and is not used in this analysis. The following sections discuss the design and performance details of the muon systems.

\begin{tabular}{lccc}
\hline & CMU & CMP & CMX \\
\hline Pseudorapidity coverage & $|\eta| \leq \sim 0.6$ & $|\eta| \leq \sim 0.6$ & $\sim 0.6 \leq|\eta| \leq \sim 1.0$ \\
Drift tube cross-section & $2.68 \times 6.35 \mathrm{~cm}$ & $2.5 \times 15 \mathrm{~cm}$ & $2.5 \times 15 \mathrm{~cm}$ \\
Drift tube length & $226 \mathrm{~cm}$ & $640 \mathrm{~cm}$ & $160 \mathrm{~cm}$ \\
Maximum drift time & $800 \mathrm{~ns}$ & $1.4 \mu \mathrm{s}$ & $1.4 \mu \mathrm{s}$ \\
Scintillation counter thickness & & $2.5 \mathrm{~cm}$ & $1.5 \mathrm{~cm}$ \\
Total counters & & 269 & 324 \\
Pion interaction lengths & 5.5 & 7.8 & 6.2 \\
Minimum detectable muon $p_{T}$ & $1.4 \mathrm{GeV} / \mathrm{c}$ & $2.2 \mathrm{GeV} / \mathrm{c}$ & $1.4 \mathrm{GeV} / \mathrm{c}$ \\
Multiple scattering resolution & $12 \mathrm{~cm} / p$ & $15 \mathrm{~cm} / p$ & $13 \mathrm{~cm} / p$ \\
\hline
\end{tabular}

Table 3.4: Design specifications of the CMU, CMP, and CMX detectors

\section{CMU: Central Muon Detector}

The CMU detectors are embedded directly behind the hadronic calorimeter wedges at about $3.5 \mathrm{~m}$ from the beam line in the full $2 \pi$ azimuthal range and extend in the pseudorapidity region $|\eta| \leq \sim 0.6$ [58][59]. The CMU uses as its shielding the calorimeter lead and iron which comprise about $5.5 \lambda$ of absorber material. The Run II CMU is comprised of the original set of Run I muon drift chambers, which is comprised of 144 modules (Figure 3.10) each containing 4 layers of 4 rectangular drift cells for a total of 16 per module. The CMU is subdivided into $12.6^{\circ}$ wedges, each containing three $2.4^{\circ}$ modules, and is built to correspond to the central calorimeter towers thus leaving a $2.4^{\circ}$ gap between each CMU wedge. The cells (shown in Figure 3.9) are $6.35 \mathrm{~cm}$ wide $\times 2.68 \mathrm{~cm}$ high $\times 226 \mathrm{~cm}$ long with respect to the beam line. At the center of each cell and running along the entire $z$-direction is a $50 \mu \mathrm{m}$ stainless steel sense wire.

The CMU cells are stacked so that there is a $2 \mathrm{~mm} \phi$-offset between the first/third and second/fourth layers. The difference in time between the radially aligned wires, i.e. layers $\mathrm{n}$ and $n+2$, provides for a track velocity calculation for the Level-1 trigger. The offset resolves 


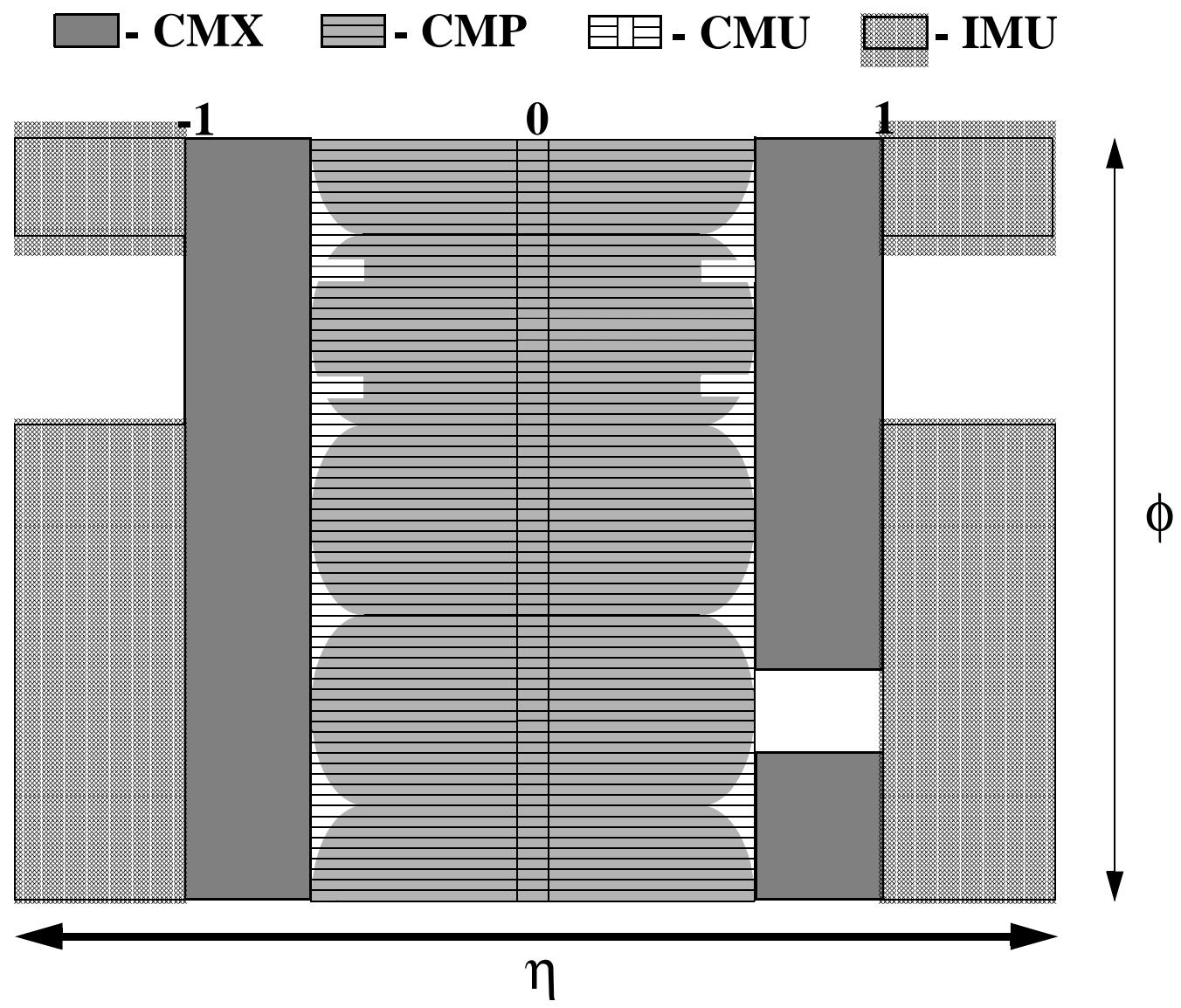

Figure 3.8: Location of the muon detector elements in the $\eta-\phi$ plane. The solid bands show the Run II coverage upgrades [42].

track ambiguity in the azimuthal dimension by determining which of the 2 sets of sense wires were hit first. Figure 3.11 shows the cell arrangement and the drift distances for a muon travelling radially outward from the beamline. The chambers in wedge $5 \mathrm{E}$ have a shortened $z$-length to accommodate a chimney through which cryogens are fed to the solenoid.

Muons travelling radially outward from the nominal interaction point will pass through four CMU drift cell layers. A muon track segment called a "stub" is constructed if a muon is detected in at least three of the four layers. Muon stubs are a type of constructed object universal to all the muon chamber systems and are used to extrapolate tracks from the SVXII or COT. In order to minimize the number of electronics channels and to allow the readout to occur only at one end of chamber, the sense wire in each cell is connected at the $\eta=0$ end to a sense wire in another cell in the same layer which is separated from the first by an intervening cell. The read-out electronics are mounted to the $\theta=56^{\circ}$ end. 


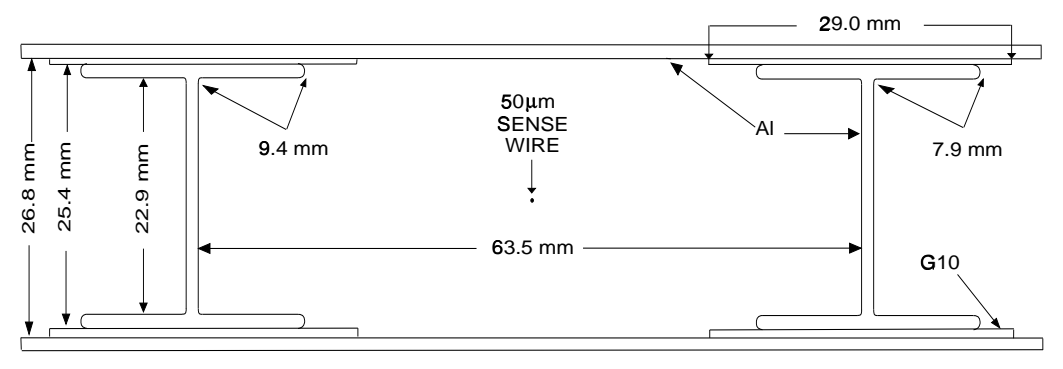

Figure 3.9: A schematic of a CMU drift cell [42].

The chamber voltages have been lowered with respect to Run I to improve the lifetime and operational efficiency. To compensate for the lower voltage, pre-amplifiers are mounted to the chambers and send analog signals to Amplifier-Shaper-Discriminator (ASD) cards located in the collision hall. These cards then send digitized differential signals to TDC's in the first floor counting room where Level-1 decisions are made.

The $z$-position of the muon stub is determined through charge division of the charge pulses reaching the ASD's at opposite ends of the connected sense wires. The reconstructed $z$-position has less resolution than Run I due to the lower chamber voltage. The CMU has a resolution of $250 \mu \mathrm{m}$ in the azimuthal direction around the sense wire, and the $z$ resolution is about $1.2 \mathrm{~mm}$.

\section{CMP: Central Muon Upgrade}

The CMP is a second set of single wire drift chambers located behind the CMU and covering a similar pseudorapidity region as shown in Figure 3.8. The variation in the pseudorapidity region along the azimuth is due to its fixed length in $z$ such that the drift chambers form a rectangular geometry about the CDF II. The solenoid return yoke provides steel absorption above and below the central detector. On either side of the central detector retractable steel absorber walls having a thickness of $60 \mathrm{~cm}$ are positioned between the $\mathrm{CMU}$ and CMP to provide absorption for the north and south side CMP walls.

The CMP walls have four layers of rectangular single-wire drift tubes. The first and third chamber cells are staggered by a half-cell width with respected to the perpendicular 


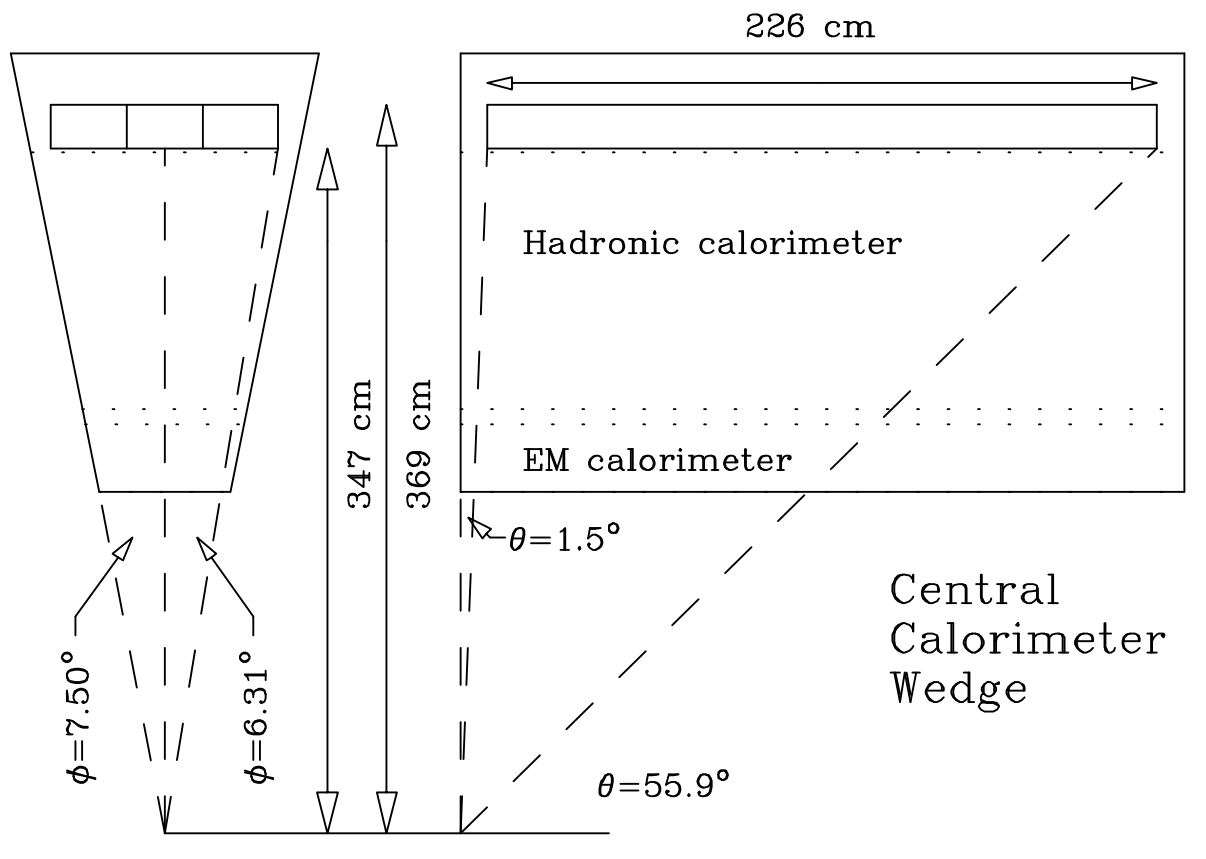

Figure 3.10: A schematic of an entire CMU module [42].

direction of the sense wire. The chambers use a 50/50 mixture of argon-ethane gas bubbled through a $-5^{\circ} \mathrm{C}$ bath of isopropyl alcohol and have a maximum drift time of about $1.4 \mu \mathrm{s}$. Due to the length of the sense wire, $6.4 \mathrm{~m}$, there is a wire support at the chamber length midpoint. The CMP provides information only in the azimuthal direction. There is no $z$ coordinate or $p_{T}$ information. The CMP does send Level-1 triggers on the detection of clusters of hits, and reduces the number of fake muon events when the clusters are paired with the CMU stubs.

On the outside of the drift chambers is a layer of rectangular scintillation counters (the CSP) read out by single phototubes. A single scintillator has dimensions of $2.5 \mathrm{~cm} \times 15 \mathrm{~cm}$ $\times 320 \mathrm{~cm}$ which covers two CMP drift chambers in width and half a chamber in length. The phototubes are located at the center of a CSP array and are displaced away from the wall to allow inter-leaving of the phototubes. This minimizes the space occupied by the light-guide and photomultiplier tube assembly. An illustration of the configuration of steel, chambers and scintillators are shown in Figure 3.12. The counters provide precise timing for track matching and beam crossing identification.

The Run I CMP covered only $76 \%$ of the complete azimuthal range due to obstruction from other detector systems and gaps in the magnetic flux return yoke which provides the hadronic shielding for the top and bottom walls. In Run II, these gaps have been filled by adding steel shielding and chambers to improve the geometric acceptance to about $93 \%$. 


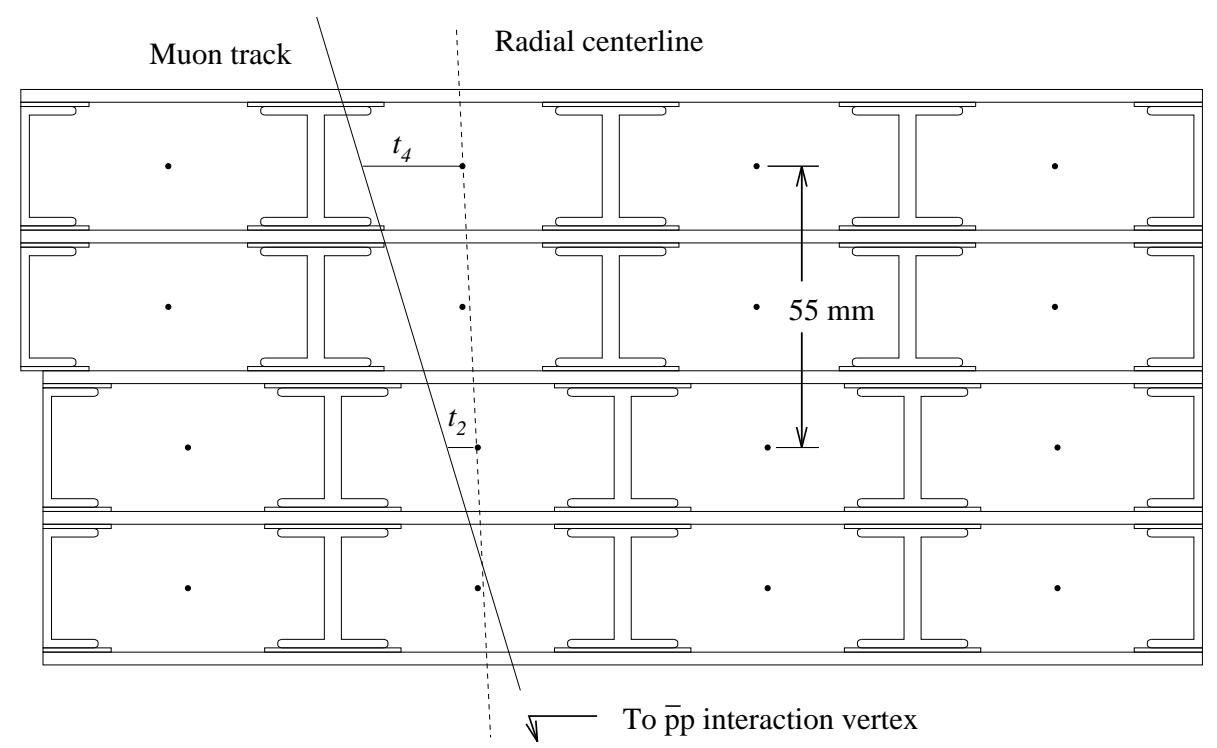

Figure 3.11: A schematic of an entire CMU module [42].

The mechanical complications and logistics of providing these additional chambers and steel absorbers are discussed at length in [42].

\section{CMX: Central Muon Extension}

The CMX detector is comprised of conical sections of drift tubes extending the central muon system from a polar angle of $55^{\circ}$ to $42^{\circ}$, or from $0.65 \leq|\eta| \leq 1.0$. The CMX is sectioned into $15^{\circ}$ sectors which are mounted on a total of four free standing arches (two east and two west) on the outside of the central detector. Each sector has eight layers of twelve drift tubes which differ from the CMP drift tubes only in their length of $1.8 \mathrm{~m}$. Due to the conical geometry, the sectors have a smaller inner edge at $|\eta|=1.0$ than the outer edge at $|\eta|=0.6$. The rectangular drift tubes are arranged such that their sense wires point radially towards the beam-line. The adjacent tubes in a layer have gaps between them so that the drift tubes can be "fanned out". The layers are overlapped so that a muon passing through the outer edge will pass through eight chambers, and a muon passing through the inner edge will pass through four, for an average of six. The first/fifth, second/sixth, etc. layers are radially aligned to determine the muon velocity for the Level-1 trigger as is done in the CMU.

The CMX drift chambers are identical to the CMP chambers in all respects with the exception that they have a shortened length of $1.8 \mathrm{~m}$. The CMX requires no additional absorber steel. Protection from the hadron calorimeter, magnet yoke, and steel from the detector end support structure provide about $6-9$ pion lengths of absorption. The CMX is 
CHAPTER 3. THE APPARATUS 


\subsubsection{Luminosity Detectors}

In Run I the integrated luminosity was measured using scintillators in the polar region $0.32^{\circ} \leq \theta \leq 4.47^{\circ}$ which classified every single crossing as either having or not having an inelastic $p \bar{p}$ collision [72]. The integrated luminosity over time $\Delta t$ was calculated as:

$$
\int_{\Delta t} L=N_{p \bar{p}} / \sigma_{i n e l}
$$

where $N_{p \bar{p}}$ is the number of inelastic $p \bar{p}$ interactions and $\sigma_{i n e l}$ is the $p \bar{p}$ inelastic cross section.

For the high instantaneous luminosities in Run II, the fraction of empty beam crossings relative to the large number of interactions is very small resulting in large relative uncertainties. To accommodate the larger Run II luminosity, the Run I scintillators have been replaced by Cherenkov Luminosity Counters (CLC) [71]. The CLC samples the beam crossings using a $1 \mathrm{~Hz}$ crossing trigger which allows for smaller relative uncertainties. The integrated luminosity is calculated using $\tilde{N}_{p \bar{p}}$, the number inelastic $p \bar{p}$ interactions as:

$$
\int_{\Delta t} L=\frac{N_{B C}}{\sigma_{\text {inel }} \cdot N_{\text {bunches }}} \sum_{i=1}^{N_{\text {bunches }}} \tilde{N}_{p \bar{p}}^{i} / N_{\text {CrossTr }}^{i}
$$

where $N_{B C}$ is the number of bunch crossings, $N_{\text {bunches }}$ is the number of bunches in the Tevatron, $N_{\text {CrossTr }}$ is the number of crossing triggers, and $i$ is the bunch index.

The counters function in the same way as the scintillator counters of Run I, and so Equation 3.1 is used to calculate the online luminosity. The advantage of using the Cherenkov Counters to sample the beam crossings is that Equation 3.2 can be used in offline analysis. These two separate methods allow for smaller systematic uncertainties in the luminosity calculation.

The CLC's are located within the $3^{\circ}$ cavities enclosed by the endplug calorimeters on the east and west sides of the central detector through which the beam-pipe extends. Each CLC is comprised of 48 conical gas-filled Cherenkov counters pointing at the interaction point and arranged about the beam-pipe in three layers of sixteen counters. Due to geometric constraints, the inner layer counters are $1.10 \mathrm{~m}$ long, and the outer two layers of counters are each $1.80 \mathrm{~m}$. Likewise the cross section of the counters increases between layers having a diameter ranging 2 to $6 \mathrm{~cm}$.

The back end which is the larger end of the Cherenkov counters is outfitted with conical mirrors made of aluminum. Photomultiplier tubes $2.5 \mathrm{~cm}$ in diameter are connected to the front end closest to the interaction point. The entire unit is mounted on the outside of a $10 \mathrm{~cm}$ diameter, aluminum tube which surrounds the beam pipe and is enlosed in a thin, aluminum pressure vessel filled with isobutane. The entire unit covers the pseudorapidity 
region $3.7 \leq|\eta| \leq 4.7$. The CLC is designed to perform reliably at a peak instantaneous luminosity of $2 \times 10^{32} \mathrm{~cm}^{-2} \mathrm{~s}^{-1}$ and has a timing resolution of $\sigma<100$ ps which is roughly a factor of 2 better than the scintillator counters used in Run I.

\subsection{Triggering and Data Acquisition}

The data collected in this analysis comes from a period of operation in which the nominal beam configuration was 36 proton bunches colliding with 36 antiproton bunches every 396 ns. This is an increase from the nominal Run I operating conditions which ran at a bunch crossing rate of $3.5 \mu \mathrm{s}$. Both the increase of the crossing rate and the increase of center of mass energy necessitated the replacement of the electronic readout system in Run II. The beam configuration was designed to run at a rate of either 108 bunch crossings every 132 ns or a lower rate of 36 bunch crossings every 396 ns. The CDF electronic readout systems are thus designed to be capable of operating at the higher bunch crossing rate, which is approximately $7.6 \mathrm{MHz}$. The specifications of the triggering system are described in this section in terms of their maximum functioning capacity.

The number of interactions per bunch crossing depends on the instantaneous luminosity. Figure 3.13 shows the number of interactions per bunch crossing as a function of the instantaneous luminosity. At an instantaneous luminosity of $2 \times 10^{32} \mathrm{~cm}^{-2} \mathrm{~s}^{-1}$ and an inelastic cross section of $\sim 60 \times 10^{-27} \mathrm{~cm}^{2}$ at $\sqrt{s}=1.96 \mathrm{TeV}$, the Run II Tevatron is expected to produce about 12 million events every second. It is not physically possible to record on to tape every event that occurs. The majority of these events are less interesting minimum bias events in which the $p \bar{p}$ collisions are diffractive and produce no secondary particles with large transverse energy. The more interesting events are typically hard scattering and result in final state particles having large transverse momentum. However these types of processes also have very small cross sections. Even at an ambitious instantaneous luminosity of $2 \times 10^{32} \mathrm{~cm}^{-2} \mathrm{~s}^{-1}$, a $200 \mathrm{GeV} / \mathrm{c}^{2}$ leptoquark will be produced at a rate of only once every 5 hours.

For these reasons, a triggering system has been put in place to determine which events are of interest and should be recorded to tape. The purpose of the triggering system is threefold: 1.) to reduce the event rate to a level suitable for writing to tape; 2.) to select events of physical interest; and 3.) to keep the number of events lost during decision-making to a minimum. There are three levels of event filtering which comprise the triggering system. Each level is given less events but more time in which to make event rejection decisions than the level preceding it. Figure 3.14 shows a flow diagram of the trigger rates. The level of sophistication in the trigger decision algorithms also increases in each level as they are afforded more time to recieve more readout from the detector. The Level- 1 decisions are 


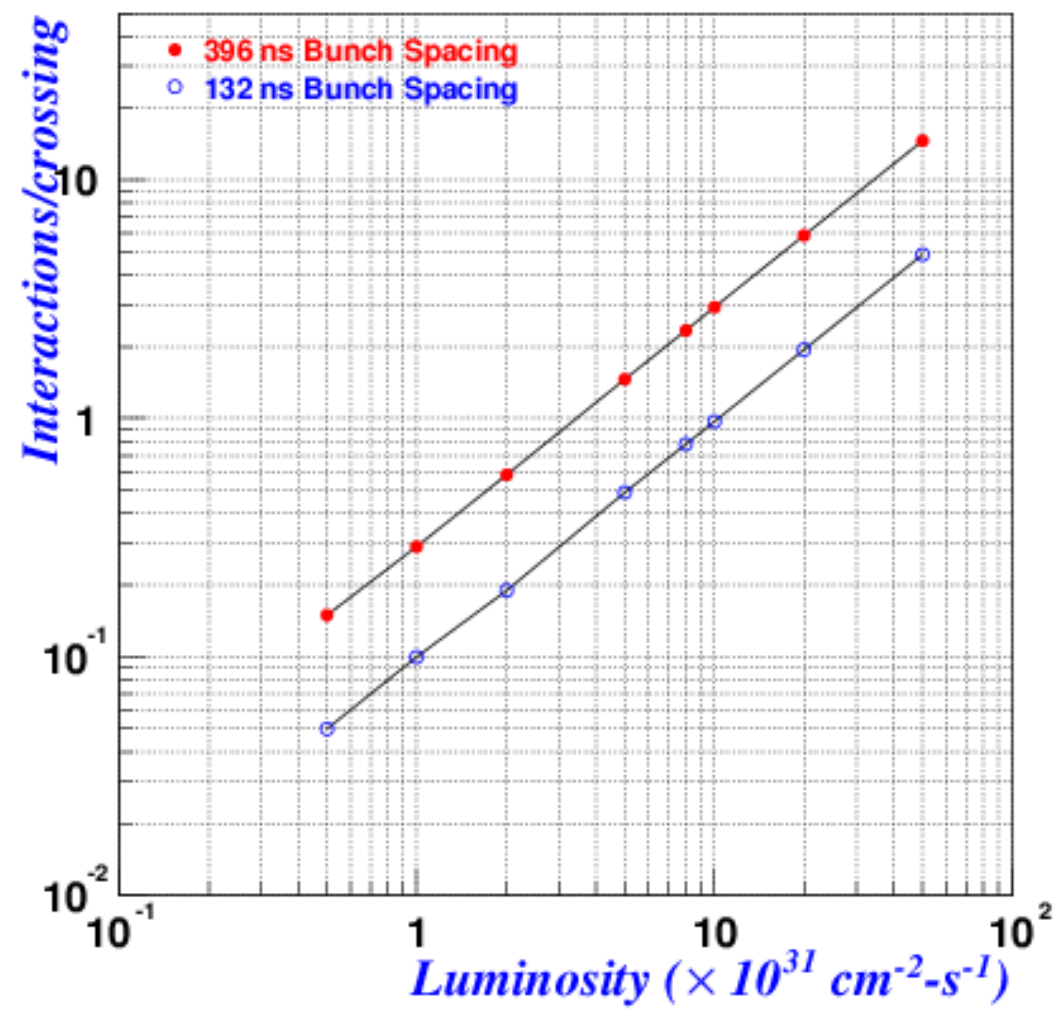

Figure 3.13: The number of interactions per bunch crossing as a function of luminosity for a bunch crossing of $132 \mathrm{~ns}$ and $396 \mathrm{~ns}$ (taken from [68]).

based on simple quantities local to a specific detector and are made in the shortest available time. Events passing the first level are sent to the second level of decision making which triggers on quantities from a larger subset of the detector. A block diagram showing the trigger components of the Level-1 and Level-2 triggers is shown in Figure 3.15. Finally, the last stage of decision making is done at Level-3 which is comprised of a farm of commercial CPU's. Each CPU is dedicated to reconstructing a single event and passes the information to a staging area before the event is written to tape. The percentage of events which are rejected solely because the triggering system is too busy making decisions on previous events is known as the "deadtime". In order to keep the deadtime at a minimum while retaining a high efficiency in collecting data for a broad range of physics, the trigger values are loaded from a table at the beginning of each data acquisition run. As the instantaneous luminosity increases with time, the trigger table is changed to keep the deadtime to a minimum. The data used in the leptoquark search was collected using inclusive muon triggers [27].

The Level-1 trigger is synchronous with the 7.6 MHz Tevatron bunch crosssing rate and 


\section{Dataflow of CDF "Deadtimeless" Trigger and DAQ}

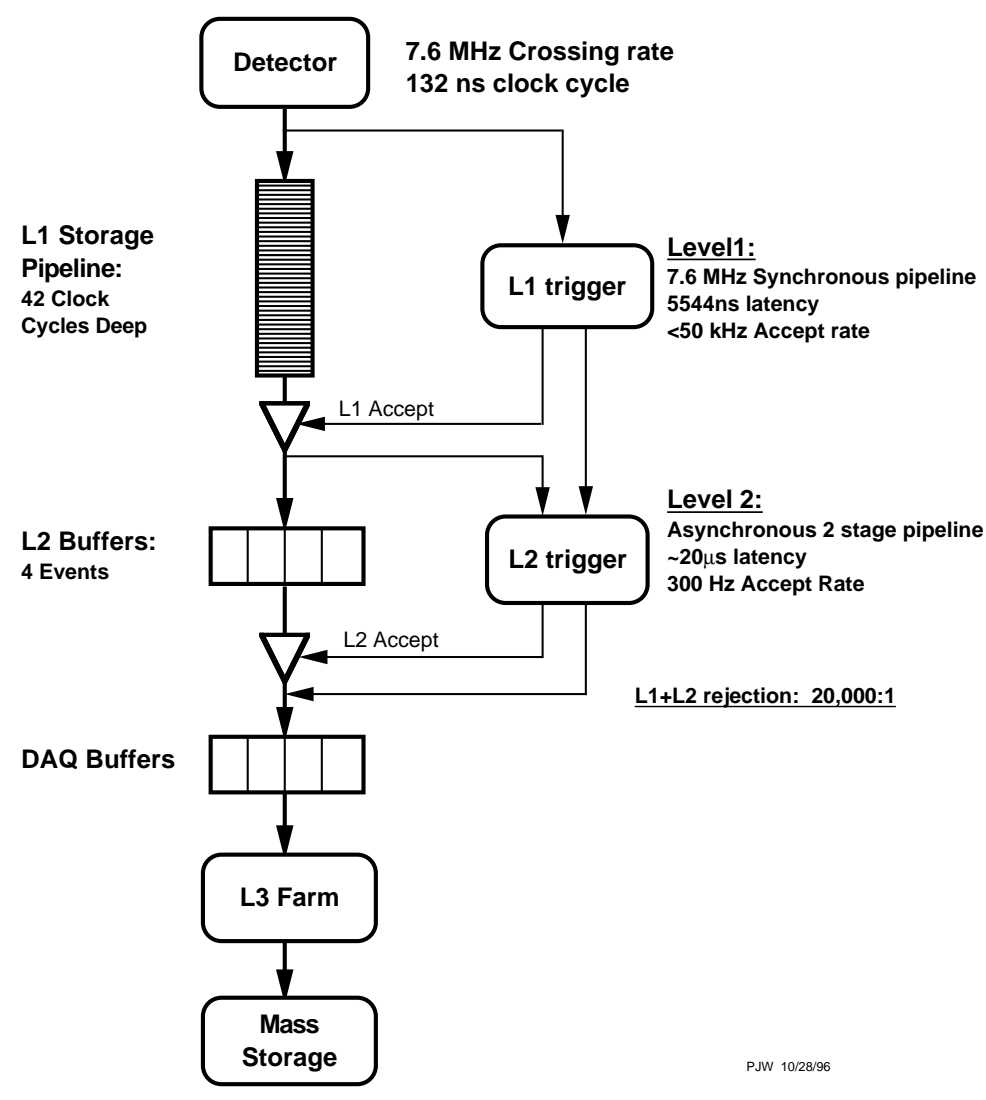

Figure 3.14: A flowchart of the CDF II online data flow. The specifications for the trigger system were designed for a maximum bunch crossing period of $132 \mathrm{~ns}$.

has a decision time of $4 \mu \mathrm{s}$. The Global Level-1 decision crate is 42 buffers deep, which means it has time to make decisions at each bunch crossing for the 132 ns crossing rate configuration. Because the system is beam synchronous, the events in $36 \times 36$ configuration are read by every third buffer. The Level-1 trigger uses an online track processor called the Extremely Fast Tracker (XFT) to identify charged high- $p_{T}$ tracks in the COT [100]. At Level-1 the tracks are linked to large EM and HAD calorimeter towers and to muon stubs in the central region. The use of pipelined central-tracking information at Level-1 was not available in Run 1. The Run II Level-1 Muon trigger has also been upgraded to use the full detector granularity in matching the muon stubs with COT tracks in order to reduce accidental triggering. For speed and simplicity, the muon data is combined into $2.5^{\circ}$ azimuthal wedges and four segments in the pseudorapidity. The track matching uses the 


\section{RUN II TRIGGER SYSTEM}

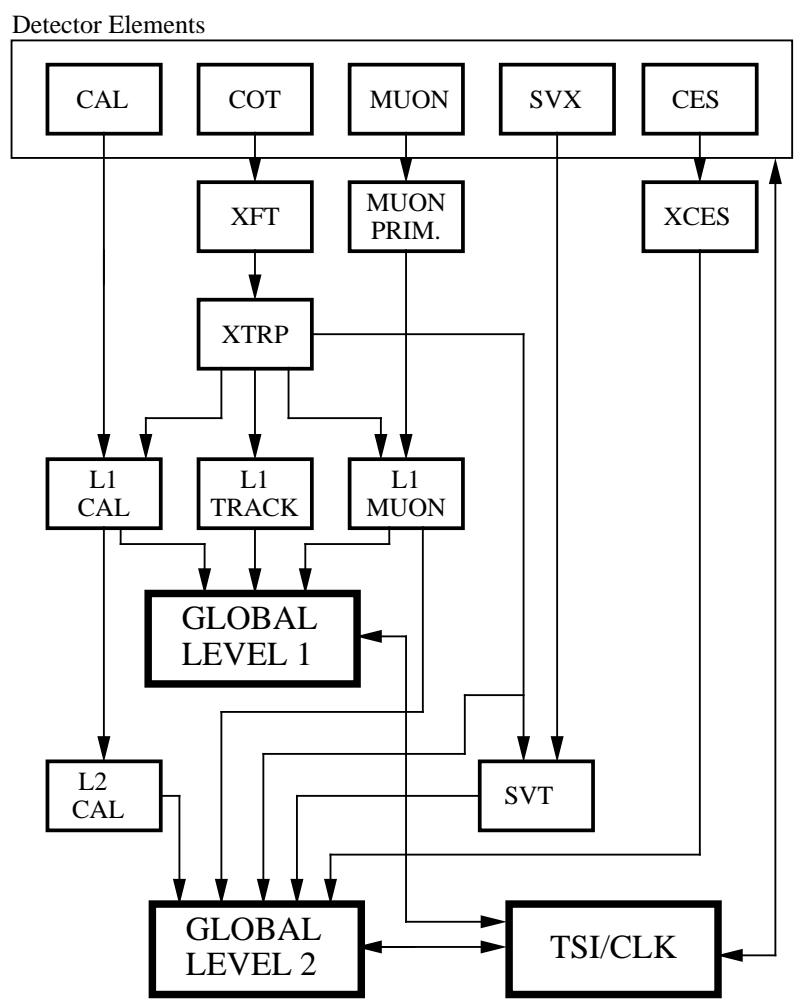

PJW 9/23/96

Figure 3.15: The Level-1 and Level-2 triggering systems. The TSI (Trigger Superviser Interface) provides an interface between the trigger system, data acquisition and clock.

full granularity in Level-2.

The Level-2 trigger is a 4-buffer deep asynchronous system having an average decision time of about $20 \mu \mathrm{s}$. The events passed from Level-1 are latched into one of the four Level2 buffers until a decision is made. The Level-2 system is capable of handling under 300 events per second. Tracking information is combined with readout from the silicon vertex tracker (SVT) which allows triggering on secondary vertices. This is most useful for $b$ physics because of the long $b$ lifetime, but is also helpful to the high- $p_{T}$ inclusive muon trigger used in this analysis. The SVT reduces the accidental rate by requiring an SVX track to point at the primary vertex, thus removing lower- $p_{T}$ muons and tightening the high- $p_{T}$ muon resolution. Photon and electron fake rates are also reduced by matching tracks to hits in the central shower max (XCES) which has a much finer granularity than the calorimeter wedge. 
Because jets are not fully contained by Level-1 trigger towers, a cluster finding algorithm is employed Level-2 which enables the system to trigger on jets.

Once an event passes the Level-2 triggers, it is sent on to the Level-3 trigger comprised of 16 subfarms each having approximately 16 commercial CPU nodes [79]. Here the events are reconstructed such that the trigger makes use of the full detector resolution. The Level-3 farm reduces the peak event rate from $300 \mathrm{~Hz}$ to about $70 \mathrm{~Hz}$, a rate suitable for writing to tape. The average size of each event ranges from 150 to $250 \mathrm{kB}$ requiring a peak bandwidth of about $17 \mathrm{MBs}^{-1}$. Each subfarm has a converter node which sends the event to the consumer server/logger which: 1.) sorts and stages the data on disks before sending them to a mass storage system; and 2.) provides and serves requests by the online consumers for data monitoring and validation [77].

The data used in this analysis was skimmed from the Stream B inclusive high- $p_{T}$ central muon dataset, and is discussed in further detail in Section 4.2. The L1, L2 and L3 triggers for this data sample are listed in Table 3.5.

\begin{tabular}{l|ll}
\hline & CMUP path & CMX path \\
\hline \hline L1 & L1_CMUP6_PT4 & L1_CMX6_PT8_PS1 \\
& & L1_CMX6_PT8_CSX_PS1 \\
& & L1_CMX6_PT8_CSX \\
\hline L2 & L2_AUTO_L1_CMUP6_PT4 & L2_AUTO_L1_CMX6_PT8 \\
& L2_TRK8_L1_CMUP6_PT4 & L2_AUTO_L1_CMX6_PT8_CSX \\
\hline L3 & L3_MUON_CMUP18 & L3_MUON_CMX18 \\
\hline
\end{tabular}

Table 3.5: The trigger paths used in leptoquark search analysis. The number following the muon type denotes the $p_{T}$ trigger of the muon stub. The number follwing the "PT" denotes the $p_{T}$ of the XFT trigger.

The names of the trigger paths reflect their trigger criteria. The number following the muon type, e.g. the L1 trigger L1_CMUP6_PT4, denotes a Level-1 $6 \mathrm{GeV} / \mathrm{c}$ trigger derived from the muon stub. The number following the "PT" denotes the muon momentum trigger from the XFT and "TRK" denotes a cut based on the track- $p_{T}$. "AUTO" denotes an automatic Level-2 accept and the "PS" denotes the implementation of a prescaled acceptance rate. 


\section{Part III}

\section{Analysis}




\section{Chapter 4}

\section{Data Sample}

In this chapter the data samples used in the leptoquark signature searches are presented. First, the strategy of the leptoquark searches is outlined. Next, the kinematic selections used in creating the inclusive high- $p_{T}$ muon data sample are presented. This is followed by a description of the generation of the simulated leptoquark signal data sample followed by a brief outline of the possible backgrounds having leptoquark signatures. Next, the technical details of the kinematic variables used in the analysis are given. Finally, the details of the efficiencies associated with preselecting events prior to cutting on these kinematic variables are presented.

\subsection{Analysis Overview and Strategy}

There are in general two types of strategies for exotics searches at high-energy particle colliders. One is model-based, in which a specific theoretical model or process is studied by examining the best signature specific to that model or process. The other is signature-based, in which a signature of interest is chosen such that it can exploit new physics independent of any particular model. In signature searches a signal region is defined with kinematic cuts kept as loose as possible. Backgrounds in the signal region are often determined by extrapolating from control regions which are exclusive to the signal region. New physics is indicated by inconsistencies found between observations in the signal region and Standard Model predictions.

The leptoquark searches employed in this analysis are signature based searches. As discussed in Section 2.6, second-generation leptoquarks can decay into $\mu q \mu q$ for $B R=1$ and $\mu q \nu q$ for $B R=1 / 2$. The quarks in any decay channel will fragment into a jet. The neutrino will pass through the CDF II detector undetected due to its neutral charge and negligible mass and will appear as an imbalance of transverse energy in the detector. Thus, we search 
for the two signatures $\mu \mu j j$ and $\mu \not_{T} j j$. Because the branching ratio for leptoquark decay is unknown, separate searches are performed for each possible final state. Both searches contain the kinematic feature of two highly energetic jets. In the $\mu \mu j j$ signature two isolated high$p_{T}$ muons are required, whereas the $\mu \mathscr{E}_{T} j j$ signature requires one isolated high- $p_{T}$ muon accompanied by a significant amount of missing transverse energy.

The number of signal events for each channel is calculated based on the data luminosity, the theoretical cross section, and the efficiency of detecting the signal event in the data. The decay product final states can be mimicked by processes not related to leptoquark pair production but which have the same or similar kinematical features. These events are referred to as background and are accounted for when performing a signal search in data. The events in the data are selected, or "cut", such that they meet requirements which distinguish them from possible background processes.

The simulated signal and background samples are produced by Monte Carlo generators which simulate physics process. The performance of the kinematic cuts are determined by generating signal and background Monte Carlo and performing an efficiency study of the selection cuts. The analysis performed in this thesis is "blind" in that these selection cuts are determined based on the Monte Carlo samples without bias from how the selection will behave in the real data.

The quality of the selection cuts is measured by their ability to successfully discriminate the signal from background while keeping a high signal acceptance. In this analysis, the selection cuts are made based on both kinematic and topological features. Also, while the majority of the background calculation is done using Monte Carlo samples, some background processes cannot be simulated. In particular, background events associated with QCD multijet processes and events involving misidentified quantities are estimated by studying the real data. An example of a misidentified quantity is a "fake" muon which is often caused by an energetic pion or jet that punches through the calorimeter and triggers a muon system.

After the selection cuts are chosen, the number of background events expected to survive the cuts in the data is estimated. The Monte Carlo is used for the shapes of the kinematic and topological variables used in the selection cuts only. The surviving background Monte Carlo events are scaled according to their theoretical cross-sections and the dataset integrated luminosity. Finally, the selection cuts are applied to the real data. A discovery is made if the number of observed events exceeds the number of expected background events, or if no excess is observed, an upper mass exclusion limit is calculated.

The selection cuts are designed to accept as many potential signal events as possible while keeping the background to a minimum. Before any selection cuts, the ratio of expected background to signal events is $\approx 1,000: 1$. As a result, keeping the selection efficiencies as high as possible is especially important for leptoquark searches so that a signal is experimentally 
accessible at the Run II energy of $\sqrt{s}=1.96 \mathrm{TeV}$.

\subsection{The Inclusive High- $p_{T}$ Data Sample}

The large amount of data taken at CDF II require that the datasets be broken up into a manageable size according to general selection criteria based on the constructed physics objects in each event. One such dataset is the Level-3 trigger dataset for high- $p_{T}$ muons located on tape with catalogue identifier bhmu08/09. This dataset was reconstructed using offline CDF II software version 4.8.4 and is comprised of data collected from March 2002 to October 2003 [17].

The data sample used for this analysis is a filtered version of the bhmu08 dataset stripped for the CDF Top Group from the inclusive high- $p_{T}$ muon datasets. The events were filtered using a loose set of muon cuts listed in Table 4.1 to keep a high efficiency for events containing high- $p_{T}$ muons. For the triggered muons the full central muon detector is used which is comprised of the following three separate muon detectors: CMU, CMP, and CMX. The L3 trigger names are MUON_CMUP18* and MUON_CMX18*, where the "** denotes a wildcard character. The resulting inclusive muon datasets are located on tape with catalogue dataset identifier btop01g for data taken from March 2002 to January 2003 and btop01j for data taken from February to October of 2003.

\begin{tabular}{ll|l}
\hline tcl switch & Inclusive Muon Criterion \\
\hline \hline - & minPtCut set 18.0 & $p_{T}>18 \mathrm{GeV} / \mathrm{c}$ \\
- & useSlidingEmCut set true & $E_{E M}<\max (2,2+0.0115(p-100)) \mathrm{GeV}$ \\
- & useSlidingHadCut set true & $E_{H A D}<\max (6,6+0.0280(p-100)) \mathrm{GeV}$ \\
- & maxCMUdXCut set 5.0 & $\Delta x(\mathrm{CMU})<5.0 \mathrm{~cm}$ \\
- $\operatorname{maxCMPdXCut~set~} 10.0$ & $\Delta x(\mathrm{CMP})<10.0 \mathrm{~cm}$ \\
- $\operatorname{maxCMXdXCut~set~} 20.0$ & $\Delta x(\mathrm{CMX})<20.0 \mathrm{~cm}$ \\
- $\operatorname{maxBMUdXCut~set~} 50.0$ & $\Delta x(\mathrm{BMU})<50.0 \mathrm{~cm}$ \\
\hline
\end{tabular}

Table 4.1: The level-3 trigger criterion used to filter the bhmu08 dataset.

A REMAKE version of btop $01 \mathrm{~g} / \mathrm{j}$ was made with software version 4.11 .1 where all the calorimeter-dependent objects, electron reconstruction objects, and muon reconstruction objects were dropped. The 4.8.4 tracks were refit using TrackRefitModule without L00 hits, and electron and muon objects were remade picking up the refitted tracks and run-dependent calorimeter corrections. Lastly, the data set was formatted into files readable by ROOT-a data analysis framework and $\mathrm{C}++$ interpreter command program [20].

The final data sample corresponds to an integrated luminosity of $197.9 \pm 11.7 \mathrm{pb}^{-1}$ (roughly twice as large as the Run integrated luminosity) and contains good runs from 141544 to 
168889 . This luminosity figure contains a correction factor of 1.019 to account for a measured proton-antiproton inelastic cross section of $60.7 \mathrm{mb}$ [56]. The good run list was selected following the Good Run List v4 created by the CDF Data Quality Group [98]. Runs were selected based on the muon detector only. Good showermax and good silicon status were not required. For the CMX detector, the good run list is divided into two sections, preCMX and post-CMX. The luminosity with and without the CMX detector in the run is $\mathscr{L}($ pre-CMX $)=19 \mathrm{pb}^{-1}$ and $\mathscr{L}($ post-CMX $)=179 \mathrm{pb}^{-1}$ respectively.

\subsection{Leptoquark Signal Monte Carlo}

For the $\mu q \mu q$ channel we generated nine 5000-event samples of leptoquark pairs decaying into $\mu q$ with leptoquark masses $M_{L Q}$ ranging from 160 to $320 \mathrm{GeV} / \mathrm{c}^{2}$. The leptoquarks have charge $-2 / 3$ and are forced to decay as $L Q \overline{L Q} \rightarrow \mu^{-} \bar{s} \mu^{+} s$. This decay mode corresponds to the BWR leptoquark model $\tilde{S}_{1 / 2}^{L}$, as shown in Table 2.1. For the $\mu \nu q q$ channel, we generated nine 5000-event samples of leptoquark pairs decaying as $L Q \overline{L Q} \rightarrow \mu^{-} c \nu_{\mu} s$. These leptoquarks belong to the $S_{0}^{L}$ model and have a charge of $-1 / 3$.

For both channels the events were generated using Pythia [96] and CDF software base release 4.9.1. The samples were generated to simulate realistic beam conditions by emulating run number 151435 and using the following talk-to for the full beam position:

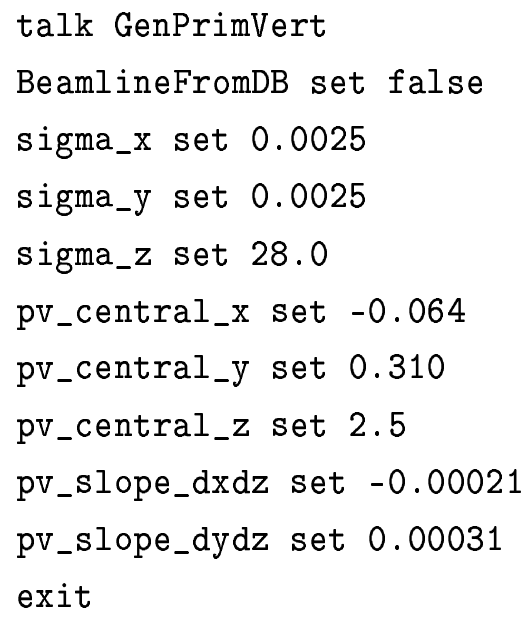

All of the scalar leptoquark Monte Carlo samples have been generated at Leading Order with $Q^{2}=M_{L Q}^{2}$ and the MRS-R2 PDF set [25]. The samples were simulated with cdfSim version 4.9.1 and were reconstructed using Production 4.9.1. 


\subsection{Background Monte Carlo}

The main sources of background for the $\mu q \mu q$ channel are Drell-Yan events associated with 2 jets where the $Z / \gamma^{*}$ decay into 2 muons, top production where both $W \rightarrow \mu \nu$, events due to QCD processes and fake muons, and cosmic ray events accompanied by jets due to radiation. The main sources of background for the $\mu q \nu q$ channel are $W$ events with 2 jets where the $W$ decays to $\mu \nu, t \bar{t}$ production decaying into the lepton + jets channel, Drell-Yan and $t \bar{t}$ production decaying to the dimuon channel in which one of the muons is mismeasured, and QCD events. Other backgrounds for these leptoquark decay channels include $b \bar{b}, Z \rightarrow \tau \tau$, and $W^{+} W^{-}$but have negligible contributions after kinematic selection.

Details of the generation of the background samples and specifics regarding the estimated background calculation in the $\mu q \mu q$ and $\mu q \nu q$ channels are discussed in Sections 5.3 and 6.3 respectively.

\subsection{Jets}

The jets used in this analysis are reconstructed using the JetCluModule with a jet clustering cone-radius in $\eta-\phi$ of 0.7 . Only jets having a pseudorapidity less than 2.0 are considered. "Tight" candidate jet events are required to have an $E_{T}>30 \mathrm{GeV}$ and "loose" are required to have $E_{T}>15 \mathrm{GeV}$. Tight jets are a subset of loose jets. The jet energies can be distorted due to various underlying and physical effects. The raw jet energies are thus scaled to account for these effects in order to obtain the energy of the final state particlelevel jet. The following combination of corrections to the raw jet energies account for these effects (the nomenclature follows CDF convention)[99]:

- Scale stability - Corrects the jets for time dependent changes in the calorimeter response. (Level 1)

- Relative - Corrects the jets for uniformity along $\eta$. This scale is determined by measuring transverse energy imbalances for dijet events. (Level 2)

- Energy scale - Corrects the jet scale for differences between Run I vs. Run II data and Run II data vs. Monte Carlo. Since photons are well measured, the jets are tuned by studying photon + jet imbalances in transverse energy. (Level 3)

- Multiple interactions - Corrects the jets for events with multiple interactions for the same bunch crossing. The contributions from the extra interactions are subtracted on average. (Level 4)

- Absolute - Corrects the jet energy measured in the calorimeter for any non-linearity and energy loss in the un-instrumented regions of each calorimeter. (Level 5) 
- Underlying event - Corrects for the energy associated with the spectator partons in a hard-scattering event. The energy is subtracted on average. (Level 6)

- Out-of-cone - Corrects the jet energy for leakage of radiation outside the clustering cone used for jet definition. (Level 7)

\subsection{Missing Transverse Energy, $\not_{T}$}

The presence of an outgoing neutrino in a physics event is deduced from an imbalance of transverse energy. The missing transverse energy, $\mathbb{F}_{T}$, is calculated by taking the vector sum of the energy deposited in the calorimeter towers plus the energy of the reconstructed muon. The $E_{T}$ must be corrected to exclude the muon energy deposited into the calorimeter:

$$
\begin{gathered}
E_{T}(x)=-E(x)=-\sum_{i}\left(H A D_{i}+E M_{i}\right) \cos \phi_{i}+\left(H A D_{T}^{\mu}+E M_{T}^{\mu}\right) \cos \phi_{\mu}-p_{T}^{\mu} \cos \phi_{\mu} \\
\#_{T}(y)=-E(y)=-\sum_{i}\left(H A D_{i}+E M_{i}\right) \sin \phi_{i}+\left(H A D_{T}^{\mu}+E M_{T}^{\mu}\right) \sin \phi_{\mu}-p_{T}^{\mu} \sin \phi_{\mu} .
\end{gathered}
$$

$E(x, y)$ is the sum of the hadronic $(H A D)$ and electromagnetic $(E M)$ energy deposited in the $i$ wedges of the calorimeter. Each physics event is assumed to have zero initial transverse energy and thus the $x$ and $y$ components of the $\mathbb{E}_{T}$ are negatively equated to the $x$ and $y$ components of the total transverse energy detected in the event. The muon energy in an event is added to the total transverse energy by subtracting the energy it deposits in the calorimeter and adding the reconstructed momentum.

The total transverse sum of energy is further corrected by the presence of jets in the event as discussed in Section 4.5. The jet energy corrections are accounted for in the $\mathbb{E}_{T}$ calculation.

\subsection{Muons}

Muons are characterized by their ability to travel through the detector calorimeter while leaving behind only a small amount of ionizing energy. For this reason, the various muon detectors at the CDF II have been judiciously positioned around the outer-most region of the detector. For large leptoquark masses, we expect the muons to be highly energetic and to travel transverse to the beam direction. There are three muon systems used in this analysis: CMU, CMP, and CMX. Each is located in the central pseudorapidity region (see Section 3.2.3).

In this analysis, the muon system whose corresponding stub is assigned to a muon track determines that muon's "type". For the muon in the $\mu q \nu q$ channel, two varieties of 
muon types are considered: CMUP and CMX. A CMX muon contains a stub hit from the CMX muon system, while a CMUP muon contains stubs hits from both the CMU and CMP detectors. For the CMUP muon, a stub hit in both muon systems is required to be associated to a CMUP muon track. The CMU and CMP muon systems nearly share the same pseudorapidity coverage and the combination of CMU and CMP stubs greatly reduces fake muon rates. For the $\mu q \mu q$ channel, we accept one of the two required muons if it is neither CMUP nor CMX. These muons are refered to as TRK muons and are similar to the CMIO tracks used in Run I.

In the CDF Run II framework, there are two choices of offline reconstructed muon objects one can use for analysis. The first are called "beam-constrained COT tracks" and are used by the CDF Electroweak Group. These muon tracks are fitted using COT hit information only and then are refitted using information on the run-dependent beam location. As a result, the muon momentum resolution is highly dependent on the beam location. The other choice of muon object and the one used in this analysis are "DefTrks", used by the CDF Top Group and are a collection of tracks stored in the PAD tracks collection. DefTrks tracks are fitted using hit information from the silicon detectors and thus do not rely on the run-dependent beam location.

The curvature of the muon tracks is corrected as prescribed in CDF Note 6558 [84]. The rate of false curvature was determined by studying the $E / p$ distributions as a function of $\phi$ for positive and negative tracks in the data. The difference in the $E / p$ for negatively and positively charged tracks is fitted and the curvature correction is determined to be:

$$
\frac{1}{q \cdot p_{T}}=\frac{1}{q \cdot p_{T}}-0.00039-0.00129 \cdot \sin (\phi+0.47)
$$

where $q$ is the charge and $p_{T}$ is the transverse muon momentum. Only tracks with silicon are corrected. Therefore we apply the correction to muon tracks in which the error on the impact parameter is $\sigma(d 0)<0.33$. This requirement indicates the presence of silicon in the track reconstruction. We observe an increase in resolution in the data for dimuon events in the mass range $76<M_{\mu \mu} / G e V / c^{2}<106$. The $Z$-mass resolution of the uncorrected data relative to the Monte Carlo increases by approximately $10-12 \%$ when making this curvature correction.

\subsection{Preselection Efficiencies}

The event-type efficiency for candidate events in the $\mu q \mu q$ and $\mu q \nu q$ channels consists of a combination of individual preselection cuts applied to the data. Together these event selection criteria ensure that the muon in the event is properly identified, is well isolated, 
and is the product of a beam-collision-related physics event. A summary of the individual preselection efficiencies for each muon type is shown in Table 4.2 and is discussed in detail in the following sections.

\begin{tabular}{|c|c|c|c|}
\hline & CMUP & CMX & TRK \\
\hline$\epsilon_{\text {muID }}$ & $88.9 \pm 0.6 \%$ & $95.2 \pm 0.5 \%$ & $94.0 \pm 0.1 \%$ \\
\hline$\epsilon_{\text {muIso }}$ & $98.2 \pm 0.3 \%$ & $97.7 \pm 0.6 \%$ & $97.8 \pm 0.0 \%$ \\
\hline$\epsilon_{\text {trig }}$ & $88.7 \pm 0.7 \%$ & $95.4 \pm 0.6 \%$ & - \\
\hline$\epsilon_{\text {muReco }}$ & $94.5 \pm 0.6 \%$ & $99.2 \pm 0.3 \%$ & - \\
\hline$\epsilon_{\text {fid }}$ & \multicolumn{3}{|c|}{$95.1 \pm 0.1($ stat $) \pm 0.5($ sys $) \%$} \\
\hline
\end{tabular}

Table 4.2: Efficiency summary table as from Ref. [80], [90], [95], and Appendix A.

\subsubsection{Muon Identification and Isolation Efficiency}

The CMUP and CMX muon identification and isolation cuts are extracted from an efficiency study on $Z \rightarrow \mu^{+} \mu^{-}$events in the same 4.11.1 data set described in Section 4.2 [80]. The results of the study, which uses beam constrained COT tracks instead of DefTrks, are reported in Table 4.2 for CMUP and CMX muons. A study of the muon ID and isolation efficiencies using DefTrks can be found in Appendix A and are the origin of the TRK muon efficiencies in this analysis, also found in Table 4.2.

A summary of the selection is shown in Table 4.3. A description of the variables used in

\begin{tabular}{|l|l|l|}
\hline Cut Variable & Purpose & Condition \\
\hline Axial,Stereo COT hits & track quality & 16 Axial, 20 Stereo Hits \\
\hline$|\Delta x|(\mathrm{CMU})$ & Track-Stub match & $<3 \mathrm{~cm}$ (if CMUP) \\
\hline$|\Delta x|(\mathrm{CMP})$ & Track-Stub match & $<5 \mathrm{~cm}$ (if CMUP) \\
\hline$|\Delta x|(\mathrm{CMX})$ & Track-Stub match & $<6 \mathrm{~cm}$ (if CMX) \\
\hline$E_{\text {em }} / \mathrm{GeV}$ & MIP & $<2+\max \left(0,0.0115^{*}(\mathrm{p}-100)\right)$ \\
\hline$E_{\text {had }} / \mathrm{GeV}$ & MIP & $<6+\max \left(0,0.0280^{*}(\mathrm{p}-100)\right)$ \\
\hline
\end{tabular}

Table 4.3: The muon ID selection cuts. TRK muons are not required to satisfy the track-stub match.

the muon ID and isolation selections follows.

- $|\Delta x|$ - This quantity is in units of centimeters and is the $r \cdot \phi$ distance between the fitted track path and the muon stub hit location. This cut ensures that a track is correctly associated to a muon stub hit. The stub-match requirement is less than 3 , 5 , and $6 \mathrm{~cm}$ for the $\mathrm{CMU}, \mathrm{CMP}$, and CMX detectors respectively. The value of the stub-match cut is related to the distance in $r$ of the muon detector from the event 
vertex. TRK muons are not required to pass this cut. Figure 4.1 shows the stub match distributions for leptoquark Monte Carlo.

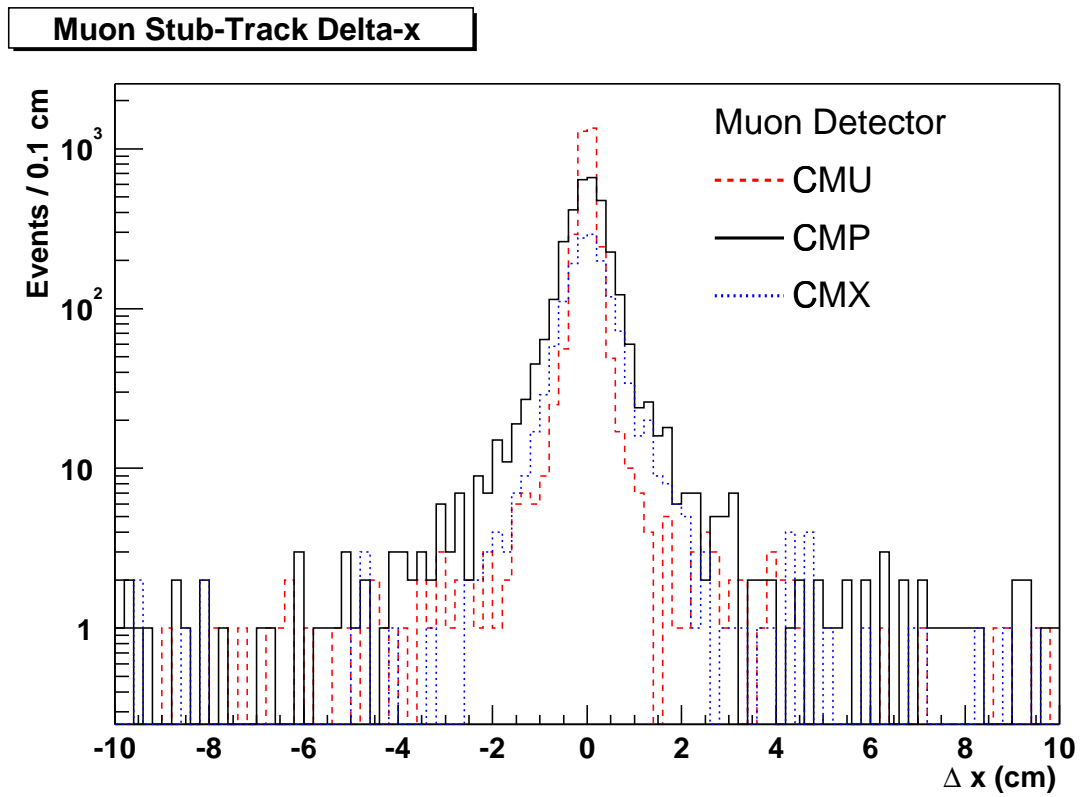

Figure 4.1: Stub-track matching for CMU, CMX, and CMX muon types in the $M_{L Q}=220$ $\mathrm{GeV} / \mathrm{c}^{2}$ Monte Carlo sample.

- $E_{E M}$ and $E_{H A D}$ - These are the energies deposited in the electromagnetic and hadronic calorimeters. The relatively large muon mass allows it to pass through the detector without causing significant reduction in its momentum. Such particles are referred to as minimum ionizing particles (MIP's). Because the amount of energy deposited in the calorimeter is dependent on the particle momentum, the $E_{E M}$ and $E_{H A D}$ selection cuts slide with the muon momentum. For $p_{T}<100 \mathrm{GeV} / \mathrm{c}$, the cuts are $E_{E M}<2 \mathrm{GeV}$ and $E_{H A D}<6 \mathrm{GeV}$. For $p_{T}>100 \mathrm{GeV} / \mathrm{c}$, the cuts slide as $E_{E M}<2+0.0115\left(c p_{T}-100\right)$ $\mathrm{GeV}$ and $E_{H A D}<6+0.0280\left(c p_{T}-100\right) \mathrm{GeV}$. Figure 4.2 shows the $E_{E M}$ and $E_{H A D}$ distributions for leptoquark Monte Carlo.

- $\mathrm{N}(\mathrm{ST}), \mathrm{N}(\mathrm{AX})$ - The number of COT Stereo hits and COT Axial hits are required to be at least 20 and 16 respectively. By definition, a DefTrk contains at least 20 stereo and 16 axial COT hits $\mathbf{O R}$ at least 5 axial silicon hits. The purpose for applying this selection cut is thus to reject any muons which qualify as a DefTrk via the 5 axial silicon hit requirement. The effect of this cut is only felt by TRK muons. The reason for this is that because TRK muons are not required to have a stub match, their track pseudorapidity is not restricted to the central region of the CMUP and CMX 

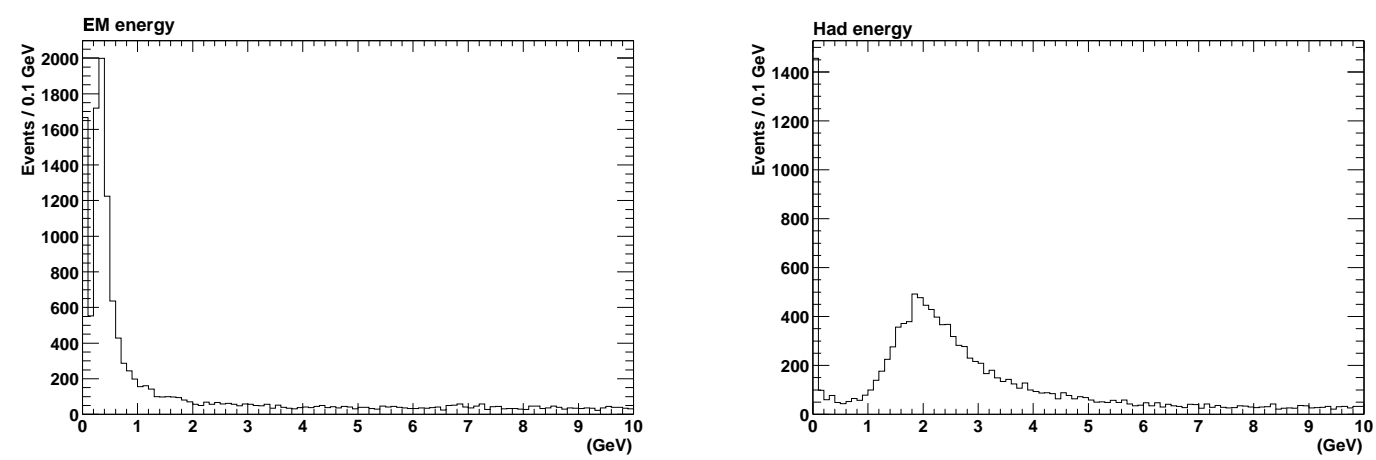

Figure 4.2: Electromagnetic and hadronic energy distributions for muons from leptoquark decay $\left(M_{L Q}=220 \mathrm{GeV} / \mathrm{c}^{2}\right)$.

detectors. The pseudorapidity range of the TRK muons is limited only by the quality of the track. For an event with a z-vertex less than $60 \mathrm{~cm}$, reconstructed TRK muons with a pseudorapidity greater than 2.0 are unlikely to pass muon selection cuts. As a result, COT hit requirements are likely to reject, for example, any forward region muon fitted to a track defined only by silicon hits and a BMU stub. The efficiency of this cut is $98.8 \%$ for TRK muons, and is $100 \%$ for CMUP and CMX muon.

- $I_{0.4} / p_{T}$ - This is the fractional isolation defined by the ratio of transverse momentum in a cone radius 0.4 about the muon and the total transverse muon momentum. This cut reduces possible muons which radiate photons via higher-order QCD processes. A well isolated muon is required to have $I_{0.4} / p_{T}<0.1$. The efficiency of this cut is listed in Table 4.2 .

\subsubsection{Muon Trigger Efficiency}

The muon trigger efficiency values are taken from [80]. The dimuon candidates were required to pass the Muon ID requirements and the efficiency was calculated by considering two separate dimuon candidates having same and opposite types. The two samples are:

- CMUP-CMX(CMX-CMUP) Dimuon events with a CMX(CMUP) trigger. The efficiency is defined as the fraction of events which also have a CMUP(CMX) trigger:

$$
\epsilon(I)=\frac{N(\text { with CMX and CMUP triggers })}{N(\text { with CMX }(\text { CMUP }) \text { triggers })}
$$


- CMUP-CMUP(CMX-CMX) Dimuon events with a CMUP trigger only. The efficiency is defined as:

$$
\epsilon(I I)=\frac{2 \cdot R}{1+R}, \quad \text { where } \quad R=\frac{N(\text { with } 2 \text { CMUP }(\mathrm{CMX}) \text { triggers })}{N(\text { with at least } 1 \mathrm{CMUP}(\mathrm{CMX}) \text { trigger })}
$$

The final trigger efficiency is calculated by combining the $\epsilon(I)$ and $\epsilon(I I)$ efficiencies in quadrature.

In June 2003, the CDF Muon Offline Group proposed a COT exit radius $\left(\rho_{C O T}\right)$ cut for CMX muon tracks. Due to the trigger design, the trigger acceptance is not the same as the detector acceptance. The XFT trigger requires a muon track to leave hits in at least 4 COT axial superlayers, but a muon track can be reconstructed offline without meeting this requirement. A cut on the $\mathrm{COT}$ exit radius, defined by the pseudorapidity and event vertex of the track, selects a detector region where the data and Monte Carlo are in agreement:

$$
\rho_{C O T}=\frac{\operatorname{sign}(\eta) \cdot z_{C O T}-z_{0}}{\tan (\lambda)}>140 \mathrm{~cm}
$$

The COT length, $z_{C O T}$, is $155 \mathrm{~cm}$ and $\lambda=\pi / 2-\theta$ where $\theta=2 \cdot \tan ^{-1}\left(e^{\eta}\right)$. The COT exit radius cut lowers the CMX muon acceptance to obtain the correct trigger efficiency. In this analysis, if a CMX muon passes the ID cuts, but fails the COT exit radius cut, then its status is demoted to a TRK posterior to the muon and event selection cuts. The efficiency of the COT exit radius cut is folded into the definition of the trigger efficiency, and the values taken from $[80]$ are from $\rho_{C O T}$ cut efficiencies.

\subsubsection{Muon Reconstruction Efficiency}

The reconstruction efficiencies for CMUP and CMX muons are scaled by the differences in Monte Carlo and data due to discrepancies in the Monte Carlo geometry description. We again use the values reported in [80] for the muon reconstruction efficiency. Cuts are made on the fiduciality of the track inside the CMX and CMUP detectors and are included in the efficiency.

\subsubsection{Z-Vertex Efficiency}

The events selected are required to have an event vertex no larger than $\pm 60 \mathrm{~cm}$ along the $z$-direction from the center of the detector. Events whose vertices are outside this range are likely to have tracks that go undetected by the silicon or even the COT tracking. However, the full luminous $p \bar{p}$ region, which is used by CLC to determine the luminosity, extends outside of this z-range. A study measuring the efficiency of this fiducial cut is reported in 
[90]. The study reports an efficiency of $\epsilon=0.951 \pm 0.005$.

\subsubsection{Cosmic Ray Rejection}

To remove the cosmic rays contaminating the sample, the di-cosmic bit is used which is based on the use of the COT timing information to deduce if the muons are compatible with an outgoing pair as one would expect in a physics process. The bit is taken from the cosmic bit word stored in the CosmicRayTaggerInfo object [97] and is $100 \%$ efficient in removing cosmic rays in dimuon events. 


\section{Chapter 5}

\section{Search for Scalar Leptoquarks in the $\mu q \mu q$ Channel}

In this chapter a search for pair-produced scalar leptoquarks decaying into $\mu q \mu q$ is presented. A preselection efficiency and a kinematic acceptance efficiency are separately calculated and then combined to determine a total leptoquark signal acceptance efficiency. An estimate of the expected number of background events in this channel is then calculated followed by a cross-check of the $Z$-boson cross section to verify that the preselection efficiencies are reliable. Next, an outline of the systematic uncertainties in the signal acceptance and data sample is presented. The chapter concludes with discussion of the method used in determining the upper limit on the leptoquark cross section. Finally, the theoretical scalar leptoquark cross section is compared to this upper limit and a new leptoquark mass exclusion limit in the $\mu q \mu q$ channel is presented.

\subsection{Event-type Efficiency Calculation}

As discussed in Section 2.6, the experimental signature for pair-produced second generation scalar leptoquarks for the $B R=1$ case consists of two isolated high- $p_{T}$ muons and two energetic jets. The full data sample is reduced by preselecting events according to the criteria described in Section 4.8. Each event is required to be a dimuon event such that at least one muon is of type CMUP or CMX and the second muon is of type CMUP, CMX, or TRK. The possible dimuon combinations are thus the following five:

- CMUP-CMUP where both the muons pass the selection criteria for a CMUP muon.

- CMX-CMX where both the muons pass the selection criteria for a CMX muon. A CMX muon is demoted to the status of TRK if it fails to pass the COT exit radius requirement. 
- CMX-CMUP where one muon passes the CMUP identification criteria and the other passes CMX.

- CMUP-TRK where one muon passes the CMUP identification criteria and the other is neither a CMUP nor CMX muon.

- CMX-TRK where one muon passes the CMX identification criteria and the other is neither a CMUP nor CMX muon.

Because the preselection efficiencies depend on the muon type, each dimuon event must be categorized separately so that the correct effficiencies can be combined for that dimuon event type. The event-type efficiencies are thus calculated using a combination of the preselection efficiencies particular to the dimuon combination. In all there are five different preselection combinations:

$$
\begin{aligned}
\epsilon_{P P} & =\left(\epsilon_{P}^{\text {trig }} \times\left(2-\epsilon_{P}^{\text {trig }}\right)\right) \times \epsilon^{\text {fid }} \times\left(\epsilon_{P}^{\text {reco }} \times \epsilon_{P}^{I D} \times \epsilon_{P}^{i s o}\right)^{2} \\
\epsilon_{P X} & =\left(\epsilon_{P}^{\text {trig }}+\epsilon_{X}^{\text {trig }}-\left(\epsilon_{P}^{\text {trig }} \times \epsilon_{X}^{\text {trig }}\right)\right) \times \epsilon^{\text {fid }} \times \epsilon_{P}^{r e c o} \times \epsilon_{X}^{r e c o} \times \epsilon_{P}^{I D} \times \epsilon_{X}^{I D} \times \epsilon_{X}^{i s o} \times \epsilon_{P}^{i s o} \\
\epsilon_{X X} & =\left(\epsilon_{X}^{\text {trig }} \times\left(2-\epsilon_{P}^{\text {trig }}\right)\right) \times \epsilon^{\text {fid }} \times\left(\epsilon_{X}^{r e c o} \times \epsilon_{X}^{I D} \times \epsilon_{X}^{i s o}\right)^{2} \\
\epsilon_{P T} & =\epsilon_{P}^{\text {trig }} \times \epsilon_{P}^{I D} \times \epsilon_{T}^{I D} \times \epsilon_{P}^{r e c o} \times \epsilon^{\text {fid }} \times \epsilon_{P}^{i s o} \times \epsilon_{T}^{i s o} \\
\epsilon_{X T} & =\epsilon_{X}^{\text {trig }} \times \epsilon_{X}^{I D} \times \epsilon_{T}^{I D} \times \epsilon_{X}^{\text {reco }} \times \epsilon^{\text {fid }} \times \epsilon_{X}^{i s o} \times \epsilon_{T}^{i s o}
\end{aligned}
$$

There is no trigger efficiency associated with TRK muons since they are not triggerable muons. Using the values from Table 4.2, we obtain for the event-type efficiencies listed in Table 5.1.

\begin{tabular}{cc}
\hline Event-type Efficiencies, $\mu q \mu q$ channel \\
\hline \hline$\epsilon_{P P}=$ & $0.637 \pm 0.011$ \\
$\epsilon_{P X}=$ & $0.718 \pm 0.011$ \\
$\epsilon_{X X}=$ & $0.807 \pm 0.011$ \\
$\epsilon_{P T}=$ & $0.631 \pm 0.008$ \\
$\epsilon_{X T}=$ & $0.761 \pm 0.007$ \\
\hline
\end{tabular}

Table 5.1: The total event-type efficiencies calculated using values from Table 4.2.

\subsection{Acceptance and Kinematical Efficiency Calculation}

Once the dimuon events have been preselected, the events are required to pass a set of selection cuts designed to discriminate the preselected events from possible backgrounds mimicking the leptoquark signal. Section 5.3 discusses the various possible backgrounds in 
the $\mu q \mu q$ channel. The simulated signal and background events used to study the acceptance of the selection cuts are discussed in Section 4.3 and 4.4. The selection cuts are applied to the data in the following order:

1. Two muons with $p_{T}>25 \mathrm{GeV} / \mathrm{c}$ - This cut ensures that the muon is a properly triggered muon and reduces the amount of QCD background associated with less energetic muons. The leading and second leading muon $p_{T}$ distributions for a leptoquark mass of $220 \mathrm{GeV} / \mathrm{c}^{2}$ are shown in Figure 5.1.
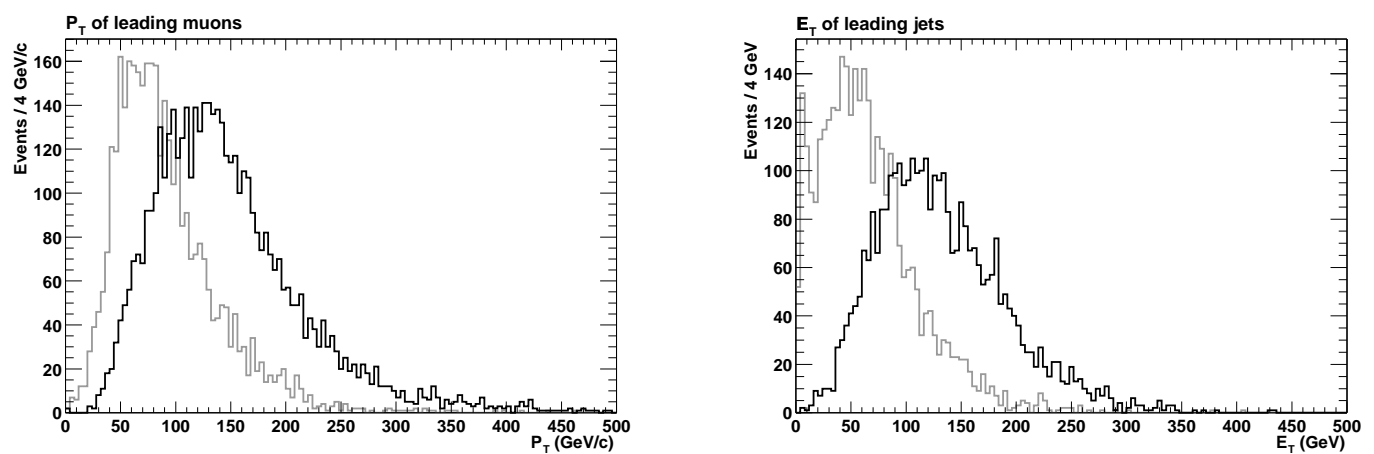

Figure 5.1: $P_{T}$ and $E_{T}$ distributions for leading (dark) and second leading (light) muons (left) and jets (right) for $M_{L Q}=220 \mathrm{GeV} / \mathrm{c}^{2}$.

2. 2 jets with $E_{T}\left(j_{1}\right)>30$ and $E_{T}\left(j_{2}\right)>15 \mathrm{GeV}$ - The requirement that both a tight and loose jet be present in the event significantly reduces the number of background events. The leading and second leading jet $E_{T}$ distributions for a leptoquark mass of $220 \mathrm{GeV} / \mathrm{c}^{2}$ are shown in Figure 5.1.

3. Removal of events with $76<M_{\mu \mu} / \mathrm{GeV} / \mathrm{c}^{2}<110$ and $M_{\mu \mu}<15 \mathrm{GeV} / \mathrm{c}^{2}$ - This cut reduces the Drell-Yan backgrounds and low energy muon events from $J / \Psi$ or $\Upsilon$. Due to the large mass of the muon, the muon curvature for high- $p_{T}$ muons is not a very reliable quantity. Therefore there is no requirement that the muons in this veto are of opposite charge.

4. $E_{T}\left(j_{1}\right)+E_{T}\left(j_{2}\right)>85 \mathrm{GeV}, p_{T}\left(\mu_{1}\right)+p_{T}\left(\mu_{2}\right)>85 \mathrm{GeV} / \mathrm{c}$

5. $\sqrt{\left(E_{T}\left(j_{1}\right)+E_{T}\left(j_{2}\right)\right)^{2}+\left(p_{T}\left(\mu_{1}\right)+p_{T}\left(\mu_{2}\right)\right)^{2} c^{2}}>200 \mathrm{GeV}$ - The combination of these last two cuts was shown in the first generation leptoquark search [86] to discriminate the leptoquark signal from both Drell-Yan and Top events. Figure 5.2 illustrates the effectiveness of the cut, where $p_{T}\left(\mu_{1}\right)+p_{T}\left(\mu_{2}\right)$ is plotted against $E_{T}\left(j_{1}\right)+E_{T}\left(j_{2}\right)$. The Drell-Yan and Top backgrounds cluster in the low energy/momentum phase space of the muons/jets. 


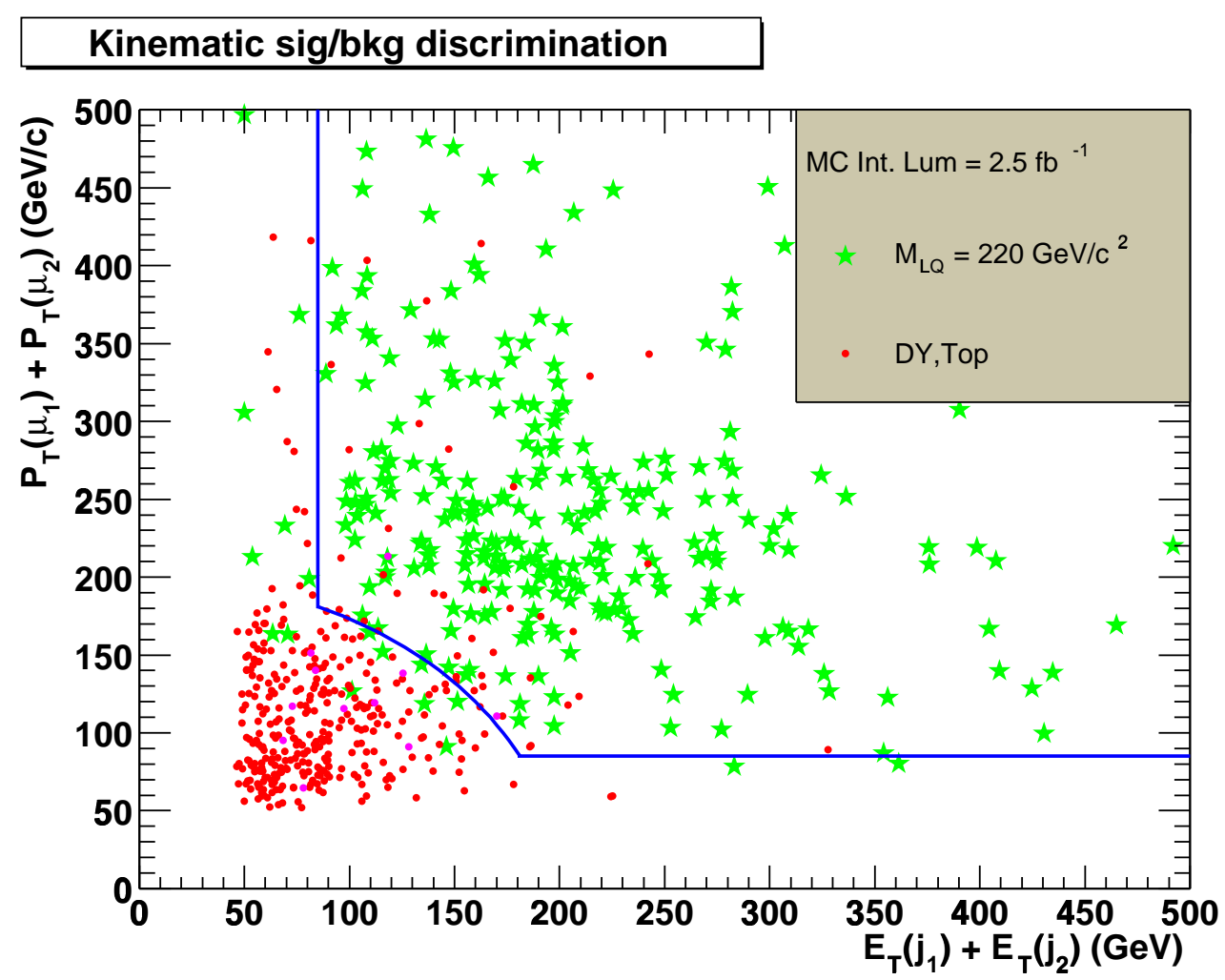

Figure 5.2: Sum of $\mathrm{E}_{T}$ (jets) versus sum of $\mathrm{P}_{T}$ (muons) for $M_{L Q}=220 \mathrm{GeV} / \mathrm{c}^{2}$ for Monte Carlo events. The plot is normalized to $2.5 \mathrm{fb}^{-1}$ and the size of the bins is $1 \mathrm{GeV}$ by 1 $\mathrm{GeV} / \mathrm{c}$.

Figure 5.3 shows the selection cut acceptances relative to the number of matched muons of type CMUP, CMX and TRK. The final selection cut acceptances are calculated relative to the total number of signal events in the Monte Carlo sample.

$$
A(L Q)=\frac{\mathrm{N}(\mathrm{CMUP}, \mathrm{CMX} \text { or TRK muons passing selection cuts })}{\mathrm{N}(\text { total events })}
$$

Since the muon ID and isolation efficiencies are calculated using real physics data, the efficiencies from these selection cuts will not be the same for simulated events. Therefore a correction factor accounting for this disparity must be applied to the signal and background Monte Carlo. Table 5.2 shows the scale factors for the leptoquark Monte Carlo for several leptoquark masses. Scale factors were also calculated for the various backgrounds.

The geometric acceptances of the muon systems is taken into account in the definition of $A(L Q)$ by selecting events having only muons of type CMUP or CMX. The geometric acceptance is related to the pseudorapidity coverage of the muon systems. In the fall of 2003, a leptoquark search in $\mu q \mu q$ was performed using $126 \mathrm{pb}^{-1}$ without the acceptance of 


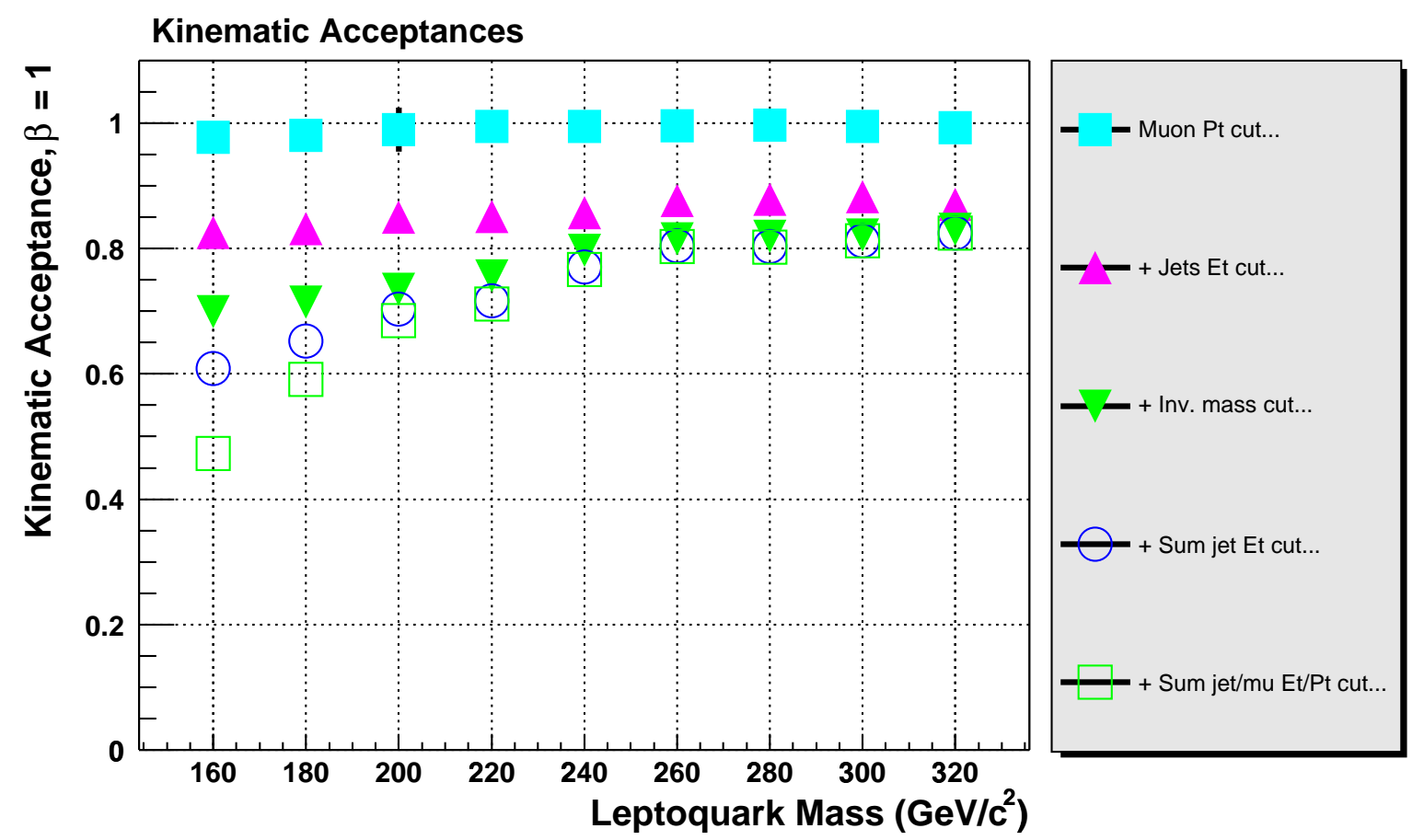

Figure 5.3: The selection cut efficiencies as a function of leptoquark mass. The top curves show the relative efficiencies of the cuts in sequence, independent of both the preselection and the geometric detector acceptance. The bottom curve shows the final efficiency with the geometric acceptance included. The statistical errors are plotted. 


\begin{tabular}{|c|c|c|c|}
\hline Mass $\left(\mathrm{GeV} / \mathrm{c}^{2}\right)$ & CMUP & CMX & TRK \\
\hline Muon ID: Data/MC \\
\hline 160 & 0.9856 & 1.0439 & 1.0450 \\
200 & 0.9867 & 1.0818 & 1.0485 \\
240 & 0.9845 & 1.0685 & 1.0715 \\
280 & 1.0125 & 1.0519 & 1.0641 \\
320 & 0.9944 & 1.0292 & 1.0690 \\
\hline Muon Isolation: Data/MC \\
\hline 160 & 1.0481 & 1.0114 & 1.0855 \\
200 & 1.0481 & 1.0188 & 1.1280 \\
240 & 1.0526 & 1.0295 & 1.1601 \\
280 & 1.0504 & 1.0010 & 1.1855 \\
320 & 1.0629 & 1.0450 & 1.2474 \\
\hline
\end{tabular}

Table 5.2: The ratios of efficiences between data and leptoquark Monte Carlo. The ratio is defined as data/MC. Scale factors calculated for the background Monte Carlo are not shown here.

TRK muons [87]. The acceptance of TRK muons in the event selection increases the total selection acceptance by roughly $200-250 \%$. The reason for this large increase in acceptance is that the TRK muons cover a larger pseudorapidity region, and hence more muons are included in the numerator of the acceptance ratio.

To calculate the total acceptance, the selection acceptance for each leptoquark mass is multiplied by the event-type efficiencies. The results are listed in Table 5.3 and are shown in Figure 5.4.

\begin{tabular}{|c|c|c|c|c|c|}
\hline $\begin{array}{l}\text { Mass } \\
\left(\mathrm{GeV} / \mathrm{c}^{2}\right)\end{array}$ & CMUP/CMUP & CMUP/CMX & CMX/CMX & CMUP/TRK & CMX/TRK \\
\hline 160 & $3.5 \pm 0.1 \pm 0.3 \%$ & $2.5 \pm 0.1 \pm 0.2 \%$ & $0.4 \pm 0.0 \pm 0.0 \%$ & $5.0 \pm 0.1 \pm 0.4 \%$ & $1.5 \pm 0.1 \pm 0.1 \%$ \\
\hline 180 & $4.2 \pm 0.1 \pm 0.3 \%$ & $2.9 \pm 0.1 \pm 0.2 \%$ & $0.5 \pm 0.0 \pm 0.0 \%$ & $6.2 \pm 0.1 \pm 0.5 \%$ & $1.8 \pm 0.1 \pm 0.1 \%$ \\
\hline 200 & $5.2 \pm 0.1 \pm 0.4 \%$ & $3.6 \pm 0.2 \pm 0.3 \%$ & $0.5 \pm 0.0 \pm 0.0 \%$ & $7.0 \pm 0.1 \pm 0.5 \%$ & $2.7 \pm 0.1 \pm 0.2 \%$ \\
\hline 220 & $4.8 \pm 0.1 \pm 0.4 \%$ & $4.5 \pm 0.2 \pm 0.3 \%$ & $0.8 \pm 0.0 \pm 0.1 \%$ & $8.5 \pm 0.2 \pm 0.6 \%$ & $2.9 \pm 0.1 \pm 0.2 \%$ \\
\hline 240 & $6.0 \pm 0.1 \pm 0.4 \%$ & $4.1 \pm 0.2 \pm 0.3 \%$ & $0.9 \pm 0.0 \pm 0.1 \%$ & $9.5 \pm 0.2 \pm 0.7 \%$ & $3.2 \pm 0.1 \pm 0.2 \%$ \\
\hline 260 & $6.4 \pm 0.2 \pm 0.5 \%$ & $4.4 \pm 0.2 \pm 0.3 \%$ & $0.8 \pm 0.0 \pm 0.1 \%$ & $11.0 \pm 0.2 \pm 0.8 \%$ & $3.2 \pm 0.1 \pm 0.2 \%$ \\
\hline 280 & $6.2 \pm 0.2 \pm 0.5 \%$ & $3.9 \pm 0.1 \pm 0.3 \%$ & $0.9 \pm 0.0 \pm 0.1 \%$ & $11.1 \pm 0.2 \pm 0.8 \%$ & $3.2 \pm 0.1 \pm 0.2 \%$ \\
\hline 300 & $6.3 \pm 0.2 \pm 0.5 \%$ & $4.6 \pm 0.2 \pm 0.3 \%$ & $0.6 \pm 0.0 \pm 0.0 \%$ & $11.2 \pm 0.2 \pm 0.8 \%$ & $3.7 \pm 0.1 \pm 0.3 \%$ \\
\hline 320 & $6.2 \pm 0.2 \pm 0.5 \%$ & $4.3 \pm 0.2 \pm 0.3 \%$ & $0.8 \pm 0.0 \pm 0.1 \%$ & $11.8 \pm 0.2 \pm 0.9 \%$ & $3.6 \pm 0.1 \pm 0.3 \%$ \\
\hline
\end{tabular}

Table 5.3: Kinematic efficiency as a function of the leptoquark mass.

After our selection cuts, two events remain in the data. In Table 5.4 we report the number of events surviving each kinematical cut. 


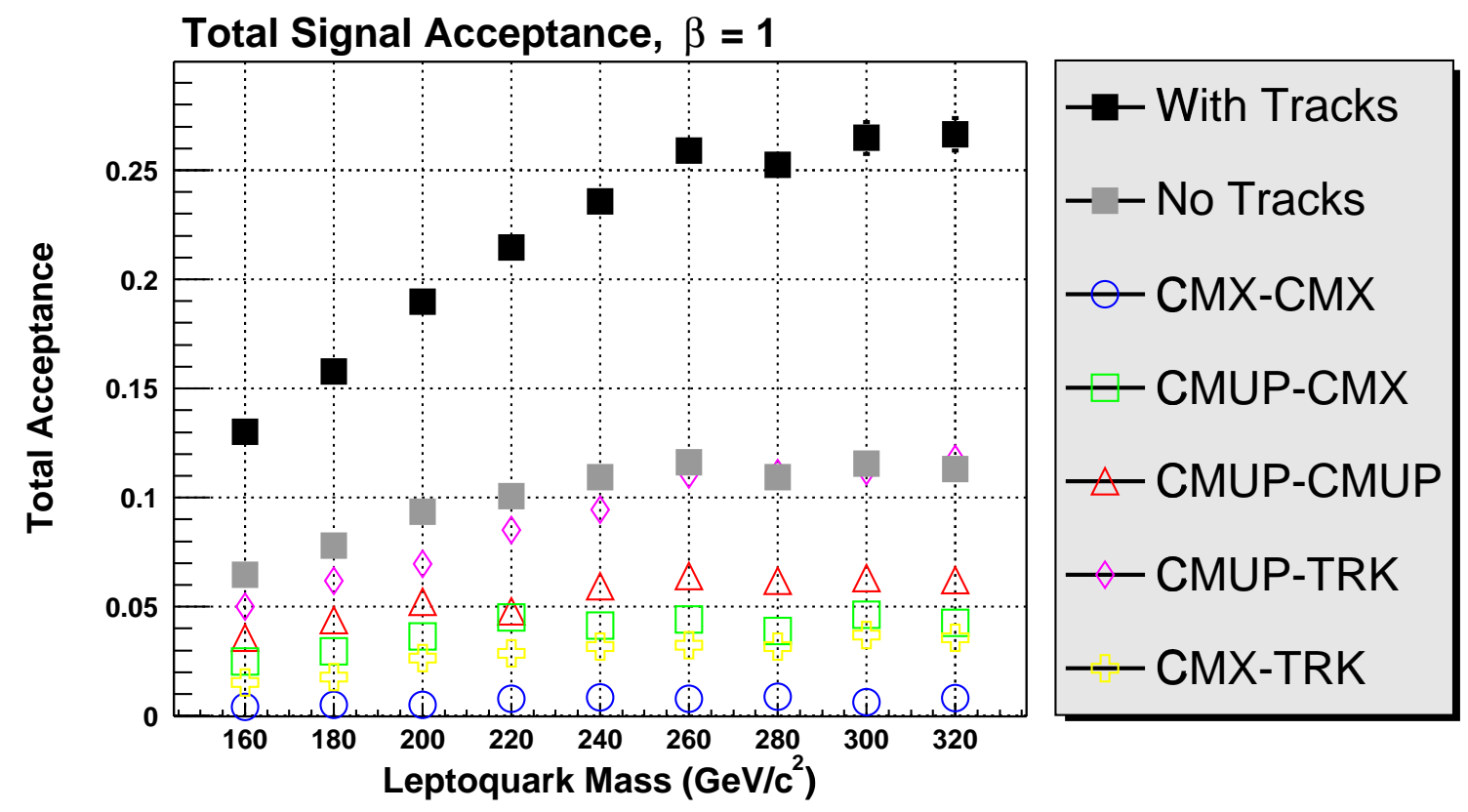

Figure 5.4: The total signal acceptance efficiencies for the $\mu q \mu q$ channel. The plot shows the contributions to the total acceptance (black) and the acceptance without TRK muons (grey) from the different muon detectors. The statistical errors are plotted. 


\begin{tabular}{|l|c|c|}
\hline Kinematic Cut & $\mathbf{N}_{\text {obs }}$ & $\mathbf{N}_{\text {same-sign }}$ \\
\hline 2 muons with $p_{T}>25 \mathrm{GeV} / \mathrm{c}$ & 8029 & 221 \\
\hline 2 jets with $E_{T}(j 1)>30$ and $E_{T}(j 2)>15 \mathrm{GeV}$ & 147 & 3 \\
\hline Removal of events with $76<M_{\mu \mu}<110$ and $M_{\mu \mu}>15 \mathrm{GeV} / \mathrm{c}^{2}$ & 26 & 2 \\
\hline$E_{T}(j 1)+E_{T}(j 2)>85 \mathrm{GeV}$ and $P_{T}(\mu 1)+P_{T}(\mu 2)>85 \mathrm{GeV} / \mathrm{c}$ & 3 & 0 \\
\hline$\left(\left(E_{T}(j 1)+E_{T}(j 2)\right)^{2}+\left(P_{T}(\mu 1)+P_{T}(\mu 2)\right)^{2} c^{2}\right)^{1 / 2}>200 \mathrm{GeV}$ & 2 & 0 \\
\hline
\end{tabular}

Table 5.4: List of events passing the selection cuts in $198 \mathrm{pb}^{-1}$ of data. The number of same-sign dimuon events found in the events passing the selection cuts is shown in the last column.

\subsection{Background calculation in the $\mu q \mu q$ channel}

The main sources of background are due to $\gamma^{*} / Z \rightarrow \mu^{+} \mu^{-}+2 j$ ets, cosmic ray events accompanied by jets due to radiation and events due to QCD/fakes. Other backgrounds from $b \bar{b}, Z \rightarrow \tau \tau, W^{+} W^{-}$are expected to be negligible due to the isolation cut and large muon and jet transverse energy $/ p_{T}$ requirements. We list the results of the background calculation in Table 5.5.

\begin{tabular}{|l|c|c|r|l|}
\hline Process & $\mathrm{N}_{\text {gen }}$ & $\sigma \cdot B R(\mathrm{pb})$ & $\mathrm{N}_{\text {exp }}$ & Comments \\
\hline$Z / \gamma * \rightarrow \mu \mu+j j$ & $159 \mathrm{k}$ & $65.5 \pm 1.7$ & $0.79 \pm 0.05$ & $10<M_{\mu \mu}<75$ \\
\hline & $138 \mathrm{k}$ & $31.4 \pm 1.0$ & $0.16 \pm 0.01$ & $75<M_{\mu \mu}<105$ \\
\hline & $30 \mathrm{k}$ & $1.04 \pm 0.03$ & $0.70 \pm 0.05$ & $105<M_{\mu \mu}<800$ \\
\hline Top Production & $40 \mathrm{k}$ & $0.07 \pm 0.01$ & $0.22 \pm 0.03$ & \\
\hline QCD/Fakes & - & - & $1.0 \pm 1.0$ & from data \\
\hline \hline Total & - & - & $2.88 \pm 1.00$ & \\
\hline
\end{tabular}

Table 5.5: Significant background processes and signal contamination.

\subsubsection{DY +2 jets}

In the Drell-Yan process, which is the largest background contributor in the $\mu q \mu q$ channel, a $Z$ or photon is produced via quark-antiquark annihilation and decays leptonically into two muons. The additional jets in such events can come from other processes or from initial/final state radiation. Though the number of these events is abundant, they are easily accounted for since the dimuon mass, $M_{\mu \mu}$, will peak approximately at the $Z$ mass, $M_{Z}$. This background is eliminated by cuts on $M_{\mu \mu}$ and the $\sum E_{T}$ cuts. Higher-order corrections allow the dimuon mass to vary however, and it is necessary to calculate the Drell-Yan + 2 jets cross section beyond leading order. For the same reason, although a dimuon mass cut is effective in removing the majority of Drell-Yan processes, there will still be a significant 
number of events having a dimuon mass shifted enough from $M_{Z}$ to evade rejection.

We studied the distribution of this background by generating the process $Z+2$ jets with ALPGEN [81] and using the Monte Carlo parton generator MCFM [70] to obtain the NLO cross section. In $198 \mathrm{pb}^{-1}$ of data, we expect $1.7 \pm 0.1$ events to be from DY +2 jets.

\subsubsection{Top quark production: $t \bar{t}$}

Another source of background is represented by $t \bar{t}$ production where both the W's decay into $\mu \nu$. We generated $5000 t \bar{t}$ events decaying into all productions using Pythia. The expected number of background events from $t \bar{t}$ is $0.22 \pm 0.03$. To normalize simulated events to data we used the theoretical cross section for $t \bar{t}, \sigma(t \bar{t}) \times B R(W \rightarrow \mu \nu)=0.0739 \mathrm{pb}$.

\subsubsection{QCD/Fakes}

The allowance of stubless muon tracks in our acceptance increases the probability of "fake" muons coming from energetic jets or pion decay which pass the trigger requirement. To estimate this signal contamination, we examine the data for same-sign events remaining after each kinematic cut. We estimate the background contribution to be twice the number of same-sign events observed. Table 5.4 shows the number of same-sign dimuon events after each kinematic cut. Since the number of same-sign events is sensitive to these cuts, we calculate the contribution from this background after making all kinematic cuts in the data. We see no same-sign events in the two events surviving selection.

To produce a more accurate estimate of this background, we loosen the kinematic cuts by removing the requirement of $P_{T}>25$ on each of the muons. This loosening creates a statistically increased set of events with which to make an extrapolation to the case of the tighter $P_{T}$ requirement. Table 5.6 shows the events remaining in the data after each of the kinematic cuts are placed.

\begin{tabular}{|l|r|r|r|r|}
\hline & \multicolumn{2}{|c|}{$P_{T}(\mu)>25$} & \multicolumn{2}{|c|}{$P_{T}(\mu)>20$} \\
\hline Kinematic Cuts & Events $\left(S_{T}\right)$ & Same-Sign $\left(B_{T}\right)$ & Events $\left(S_{L}\right)$ & Same-Sign $\left(B_{L}\right)$ \\
\hline After Muon $P_{T}$ Cut & 8027 & 220 & 8632 & 298 \\
$P_{T}(j)>15,30$ & 144 & 3 & 171 & 5 \\
Dimuon Mass Cut & 25 & 2 & 37 & 3 \\
Sum $P_{T}$ Cuts & 3 & 0 & 6 & 1 \\
Sqrt All Sum Cut & 2 & 0 & 4 & 1 \\
\hline
\end{tabular}

Table 5.6: Surviving same-sign events after each kinematic cut.

We then make a first order approximation that the relationship between these two sets 


\begin{tabular}{|l|l|l|l|l|l|}
\hline & CMUP-CMUP & CMX-CMX & CMUP-CMX & CMUP-TRK & CMX-TRK \\
\hline $\mathrm{A}(\mathrm{Z}) \%$ & $3.2 \pm 0.1$ & $0.73 \pm 0.04$ & $2.8 \pm 0.1$ & $10.4 \pm 0.1$ & $4.8 \pm 0.1$ \\
\hline$\epsilon_{\mu \mu}$ & $0.64 \pm 0.01$ & $0.81 \pm 0.01$ & $0.72 \pm 0.01$ & $0.63 \pm 0.01$ & $0.76 \pm 0.01$ \\
\hline$N_{\text {obs. }}$ & 881 & 267 & 879 & 3268 & 1546 \\
\hline$N_{\text {bkg. }}$ & 0.7 & 2.6 & 0.1 & 45.0 & 22.3 \\
\hline $\mathscr{L}, \mathrm{pb}^{-1}$ & $197.9 \pm 11.7$ & $179.2 \pm 10.6$ & $179.2 \pm 10.6$ & $197.9 \pm 11.7$ & $179.2 \pm 10.6$ \\
\hline \hline$\sigma \cdot\left(Z \rightarrow \mu^{+} \mu^{-}\right)[\mathrm{pb}]$ & $219.8 \pm 16.3$ & $252.8 \pm 25.4$ & $239.1 \pm 17.8$ & $246.7 \pm 15.8$ & $234.6 \pm 15.9$ \\
\hline
\end{tabular}

Table 5.7: Parameters used in the calculation of the $\mathrm{Z}$ cross-section.

of statistics is linear:

$$
B_{T}=\frac{S_{T} \times B_{L}}{S_{L}}
$$

where $S, B \equiv$ signal,background and $T, L \equiv$ tight,loose. Using this prescription, the predicted number of same-sign events passing all of the kinematic cuts is 0.5 . Therefore, the estimated fake background using same-sign events is $1 \pm 1$. The observed number of zero same-sign events in the data falls within these limits.

\subsection{Cross-check with the $Z$-boson cross section}

In order to check that our acceptances and efficiencies are compatible with other measurements, we check that the events left before requiring two jets and all following analysis cuts are consistent with the production of $Z$. $Z$-boson candidates are selected by requiring $75<M_{\mu \mu} / \mathrm{GeV} / \mathrm{c}^{2}<105$. The cross-section is calculated from the formula:

$$
\sigma \times B R(p \bar{p} \rightarrow Z \rightarrow \mu \mu)=\left(N_{Z}-N_{B G}\right) /\left(A_{Z} \times \epsilon_{\mu \mu} \times \mathscr{L}\right)
$$

The signal acceptance is calculated using 70k $Z$ Monte Carlo events from the zewk0m sample generated by the CDF Electroweak Group. Using the values listed in Table 5.7, we obtain for the $\mathrm{Z}$ cross-section values compatible with theory and other measurements [89] [44].

\subsection{Systematic Uncertainty}

The following systematic uncertainties are considered:

- Luminosity [56]: $6 \%$

- Acceptance 


\begin{tabular}{|l|ccccc|c|}
\hline Mass & $\mathrm{A}\left(M_{L Q}\right) \%$ & & Statistical\% & & Systematic\% & Combined Rel.\% \\
\hline 160 & 13.0 & \pm & 0.4 & \pm & 1.0 & 8.5 \\
180 & 15.8 & \pm & 0.4 & \pm & 1.2 & 8.2 \\
200 & 19.0 & \pm & 0.6 & \pm & 1.4 & 8.2 \\
220 & 21.4 & \pm & 0.5 & \pm & 1.6 & 7.9 \\
240 & 23.6 & \pm & 0.6 & \pm & 1.7 & 8.0 \\
260 & 25.9 & \pm & 0.7 & \pm & 1.9 & 7.9 \\
280 & 25.3 & \pm & 0.6 & \pm & 1.9 & 8.8 \\
300 & 26.5 & \pm & 0.7 & \pm & 2.0 & 8.0 \\
320 & 26.6 & \pm & 0.8 & \pm & 2.0 & \\
\hline
\end{tabular}

Table 5.8: The total acceptance percentages for each leptoquark mass listed with the statistical, systematic and combined relative errors.

- PDF: $4.3 \%$ (from Run I)

- Statistical Error of Monte Carlo: 2.2\%

- Jet Energy Scale: $<1 \%$

- Muon ID efficiency $[18,19]$

- muon reconstruction: $0.3 \%$

- Event vertex cut [90]: $0.5 \%$

Adding the above systematic uncertainty in quadrature will give a total systematic uncertainty of about $8 \%$. The statistical, systematic, and combined relative errors are listed in Table 5.8.

\subsection{Cross Section Upper Limit Calculation}

The number of events observed in the data for the $\mu q \mu q$ channel is consistent with the expected background. Since we do not observe a signal, the leptoquark mass region excluded by the search is calculated by calculating the upper limit on the leptoquark cross section and comparing it with the theoretical cross section for scalar leptoquark pair production. We exclude the mass region for which the theoretical cross section exceeds the observed upper limit.

The production cross section $\sigma$ of the process $L Q \overline{L Q} \rightarrow \mu q \mu q$ can be written as follows:

$$
\sigma \times B R(L Q \overline{L Q} \rightarrow \mu q \mu q)=\sigma \times \beta^{2}=\frac{N}{\epsilon_{t o t} \times \mathscr{L}}
$$


where $N$ is the number of observed data events after our selection, $\epsilon_{t o t}$ is the leptoquark mass-dependent total selection efficiency and $\mathscr{L}$ is the time-integrated measured luminosity.

The upper limit on the observed leptoquark cross section is determined by estimating the number of possible leptoquark events observed for a specific probability or confidence level (CL):

$$
\sigma^{l i m}=\frac{N^{l i m}}{\epsilon_{\text {tot }} \times \mathscr{L}}
$$

To determine the limit on the observed number of leptoquark events using the classical or frequentist approach, one could formulate the limit based on a repeated number of experiments, i.e:

$$
P\left(X^{l}\right)=\lim _{N \rightarrow \infty} \frac{N_{l}}{N}
$$

where $P\left(X_{l}\right)$ is the probability of measuring $X^{l}, N$ is the number of repeated identical experiments, and $N_{l}$ is the limit of the frequency of measuring $X^{l}$. The alternative to the frequentist approach is to use Bayesian statistics which allows for an interpretation of the probability as a degree of belief. Heuristically, Bayes' Theorem states that the probability of observing an event belonging to class $C_{k}$ after observing an event $X$ with feature $l$ is proportional to product of the likelihood of an event $X^{l}$ belonging to class $C_{k}$ and the prior probability of there being events of class $C_{k}$. The complete theorem is stated by:

$$
P\left(C_{k} \mid X^{l}\right)=\frac{P\left(X^{l} \mid C_{k}\right) P\left(C_{k}\right)}{P\left(X^{l}\right)}
$$

The probabilities $P\left(C_{k}\right)$ and $P\left(X^{l}\right)$ are the fraction of events belonging to class $C_{k}$ and having feature $X^{l}$ respectively. The probability on the left, $P\left(C_{k} \mid X^{l}\right)$, is the posterior probability. It gives the probability that a data point having a feature variable $X^{l}$ belongs to the class $C_{k}$ after a measurement of $X$. The probability $P\left(X^{l} \mid C_{k}\right)$ is the conditional probability. This function represents the probability that a given data point belonging to a class $C_{k}$ has feature $X^{l}$. This probability can be expressed as a likelihood function. The term $P\left(C_{k}\right)$ allows for the incorporation of knowledge of features of the data prior to making a determination of the classification of a measurement. Since the probability $P\left(X^{l}\right)$ is independent of the classification of a measurement, it can be seen as a normalization factor. Hence, Equation 5.6 can be summarized as:

$$
\text { posterior probability }=\frac{\text { likelihood function } \times \text { prior }}{\text { normalization factor }} \text {. }
$$


The results obtained from the two methods are not always identical (for discussion see [43]). There are several reasons for using a Bayesian versus a frequentist approach to calculate the upper limit on the number of observed leptoquark events. The first is purely aesthetic. In the Bayesian approach a posterior probability is expressed by a likelihood function modified by the result of the experience itself. By using the likelihood, a distinction is implied that given an experimental measurement $m$, what is considered is the behavior of varying the true value $m_{t}$. The frequentist approach makes no such distinction. Instead it implies that no matter what is the true value, $m_{t}$, the probability expresses the percentage of time a limit calculated by an infinite ensemble of measurements contains $m_{t}$. The second argument is pragmatic: Limits obtained by the Bayesian approach are often more conservative than the frequentist approach. This becomes especially acute in the case where errors are included. In the frequentist approach, introducing errors from the signal acceptance or background calculation actually tightens the limit result. Intuitively this is contrary to the notion that including errors should weaken the limit. Finally, for reasons not exploited in this dissertation, the Bayesian approach allows for a more elegant combination of results.

There are also arguments for not using a Bayesian approach. As pointed out by Cousins in [43], it is not always clear what should be the appropriate choice of a prior distribution when nothing is known about it. A semiclassical approach which avoids this issue is proposed in [62], however it does not incorporate the errors from both the signal acceptance and background calculation.

In this analysis we follow a Bayesian approach which incorporates errors from the signal acceptance and background calculation [11]. We calculate the posterior probability distribution for observing $n_{0}$ events with $\mu_{S}$ expected signal events and $\mu_{B}$ expected background events as,

$$
P\left(\mu_{S} \mid n_{0}, \mu_{B}\right)=\frac{\mathscr{L}\left(n_{0} \mid \mu_{S}+\mu_{B}\right) P\left(\mu_{S}\right)}{\int_{0}^{\infty} \mathscr{L}\left(n_{0} \mid \mu_{S}+\mu_{B}\right) P\left(\mu_{S}\right) d \mu_{S}},
$$

where $\mathscr{L}\left(n_{0} \mid \mu_{S}+\mu_{B}\right)$ is the likelihood distribution of observing $n_{0}$ given an expected value of $\mu_{S}+\mu_{B}$. We choose a uniform prior probability, $P\left(\mu_{S}\right)$ such that $P\left(\mu_{s}\right)=1$ for $\mu_{S} \geq 0$. As stated before, the denominator in Equation 5.7 may be regarded as a normalization factor so that Equation 5.7 can be written as:

$$
P\left(\mu_{S} \mid n_{0}, \mu_{B}\right) \quad \propto \mathscr{L}\left(n_{0} \mid \mu_{S}+\mu_{B}\right)
$$

Because the number of expected signal events is very small, the Poisson distribution is chosen for the likelihood function describing the conditional probability. If $n_{0}$ is the observed 
number of events, then in the absence of background the likelihood of observing $n_{0}$ events given that a mean number of signal events, $\mu_{S}$, are expected is given by:

$$
\mathscr{L}\left(n_{0} \mid \mu\right)=\frac{\mu_{S}^{n_{0}} e^{-\mu_{S}}}{n_{0} !}
$$

Since $n_{B}$ background events are expected in the final event selection, the likelihood needs to be modified to accommodate the possibility of other types of event classification. In this case it is straightforward to include the background:

$$
\mathscr{L}\left(n_{0} \mid \mu_{S}+\mu_{B}\right)=\frac{\left(\mu_{S}+\mu_{B}\right)^{n_{0}} e^{-\left(\mu_{S}+\mu_{B}\right)}}{n_{0} !},
$$

where $\mu_{B}$ is the expected mean number of background events and must be known exactly. However in experiments $\mu_{B}$ is not exactly known but is calculated within an overall uncertainty, $\sigma_{B}$, from statistical and systematic errors. Likewise there is an additional error associated with the signal acceptance denoted by $\sigma_{A}$. To incorporate these errors, the likelihood is "smeared" by these errors which are assumed to have a gaussian distribution. This is done by multiplying the probability distributions by gaussians having widths proportional to the corresponding signal and background acceptance uncertainties and summing over values of the expected signal and background events:

$$
P\left(\mu_{S} \mid n_{0}, \mu_{B}\right) \propto \frac{1}{2 \pi \sigma_{B} \sigma_{S}} \int_{0}^{\infty} \int_{0}^{\infty} \mathscr{L}\left(n_{0} \mid \mu_{B}^{\prime}+\mu_{S}^{\prime}\right) e^{\frac{-\left(\mu_{B}-\mu_{B}^{\prime}\right)^{2}}{2 \sigma_{B}^{2}}} e^{\frac{-\left(\mu_{S}-\mu_{S}^{\prime}\right)^{2}}{2 \sigma_{S}^{2}}} d \mu_{B}^{\prime} d \mu_{S}^{\prime},
$$

where $\sigma_{S}=\mu_{S}\left(\sigma_{A} / A\right)$. To compute the confidence level of the upper limit, we sum over the possible Poisson likelihoods. The confidence level is given by $\epsilon=1-\mathrm{CL}$, where $1-\epsilon$ is the probability of observing more than $n_{0}$ events. The upper limit on $\mu_{S}$ is defined as that value, $\mu_{S}^{l i m}$ for which we expect to observe $n_{0}$ events or fewer for a desired CL, i.e.:

$$
1-C L=\frac{\int_{\mu_{S}^{l i m}}^{\infty} P\left(\mu_{S} \mid n_{0}, \mu_{B}\right) d \mu_{S}}{\int_{0}^{\infty} P\left(\mu_{S} \mid n_{0}, \mu_{B}\right) d \mu_{S}} .
$$

In Table 5.9 we report the expected number of events of signal in $198 \mathrm{pb}^{-1}$ given the above efficiencies and the NLO theoretical cross section for different values of the renormalization/factorization scale is reported in Table 5.9. The second column shows the upper limit on the expected number of background subtracted signal events at 95\% CL.

In the $\mu q \mu q$ channel we find 2 candidate events after our selection, and thus set an upper limit on the scalar leptoquark cross section as a function of $M_{L Q}$. We calculate the upper limit on the observed leptoquark events, $N^{\text {lim }}$, at a $95 \% \mathrm{CL}$. The limit calculation was carried out using the bayes.f fortran code provided by the CDF statistics committee [11] 


\begin{tabular}{|cccc|}
\hline $\begin{array}{l}\text { Mass } \\
\left(\mathrm{GeV} / \mathrm{c}^{2}\right)\end{array}$ & $N_{95 \%}$ & $Q^{2}=M_{L Q}^{2} / 4$ & $Q^{2}=4 M_{L Q}^{2}$ \\
\hline 160 & 4.86 & 30.62 & 24.39 \\
180 & 4.85 & 17.84 & 14.31 \\
200 & 4.85 & 10.82 & 8.76 \\
220 & 4.85 & 6.41 & 5.18 \\
240 & 4.85 & 3.80 & 3.07 \\
260 & 4.85 & 2.31 & 1.85 \\
280 & 4.85 & 1.25 & 1.00 \\
300 & 4.85 & 0.74 & 0.59 \\
320 & 4.85 & 0.42 & 0.33 \\
\hline
\end{tabular}

Table 5.9: The measured upper limit on the expected number of background subtracted signal events in $198 \mathrm{pb}^{-1}$ for $L Q \overline{L Q} \rightarrow \mu q \mu q$. The right two columns show the theoretical expected number of scalar leptoquark events for different factorization/renormalization scales.

which follows the previously discussed Bayesian prescription for calculating upper limits.

In Table 5.10 we report the $\beta=1$ values of the cross-section limits in $\mu q \mu q$ for each $M_{L Q}$ and the theoretical calculations at NLO for pair production of scalar leptoquarks at the Tevatron using the CTEQ4M PDF with different choices of scale. In Figure 5.5 the

\begin{tabular}{|c|c|c|c|}
\hline Mass & 95\% CL $\sigma(\mathrm{pb})$ & \multicolumn{2}{|c|}{$\sigma$ Theory CTEQ4M $(\mathrm{pb})$} \\
\cline { 3 - 4 }$\left(\mathrm{GeV} / \mathrm{c}^{2}\right)$ & & $Q^{2}=M_{L Q}^{2} / 4$ & $Q^{2}=4 M_{L Q}^{2}$ \\
\hline 160 & 0.189 & 1.190 & 0.948 \\
180 & 0.155 & 0.571 & 0.458 \\
200 & 0.129 & 0.288 & 0.233 \\
220 & 0.114 & 0.151 & 0.122 \\
240 & 0.104 & 0.081 & 0.066 \\
260 & 0.095 & 0.045 & 0.036 \\
280 & 0.097 & 0.025 & 0.020 \\
300 & 0.093 & 0.014 & 0.011 \\
320 & 0.092 & 0.008 & 0.006 \\
\hline
\end{tabular}

Table 5.10: Values of the upper limits at 95\% CL of the production cross-section of second generation leptoquarks decaying into $\mu q \mu q$ as a function of $M_{L Q}$. The last 2 columns on the right report the result of the theoretical calculations at Next-To-Leading order with CTEQ4M for different choices of the scale multiplied by a factor of $\beta=1$.

limit cross-section as a function of $M_{L Q}$ is compared with the theoretical expectations for $\beta=1$. At the intersection point between experimental and theoretical curves we find the lower limit on $M_{L Q}$ at $221 \mathrm{GeV} / \mathrm{c}^{2}$. 


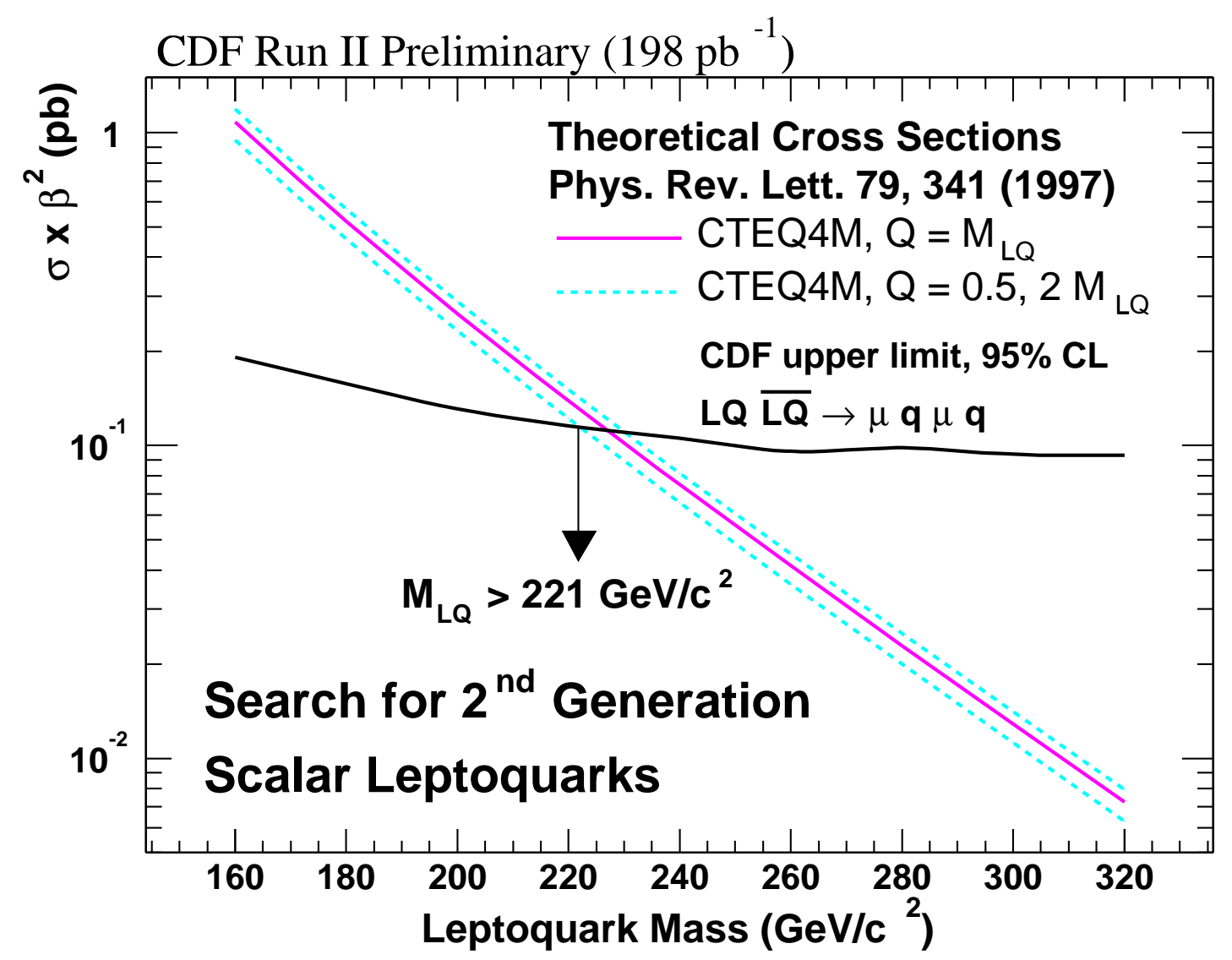

Figure 5.5: Limit cross-section as a function of $M_{L Q}$ compared with the theoretical expectations calculated at NLO accuracy. At the intersection points between experimental and theoretical curves we find a lower limit on $M_{L Q}$ at $221 \mathrm{GeV} / \mathrm{c}^{2}$. 


\section{Chapter 6}

\section{Search for Scalar Leptoquarks in the $\mu q \nu q$ Channel}

In this chapter a search for pair-produced scalar leptoquarks decaying into $\mu q \nu q$ is presented. A preselection efficiency and a kinematic acceptance efficiency are separately calculated and then combined to determine a total leptoquark signal acceptance efficiency. An estimate of the expected number of background events in this channel is then calculated followed by a cross-check of the production of $W+2$ jet events to verify that the preselection efficiencies are reliable. Next, an outline of the systematic uncertainties in the signal acceptance and data sample is presented. The chapter concludes with a calculation of the upper limit on the leptoquark cross section. Finally, the theoretical scalar leptoquark cross section is compared with this upper limit and a new scalar leptoquark mass exclusion limit in the $\mu q \nu q$ is presented.

\subsection{Event-type efficiency calculation}

The experimental signature of a second generation scalar leptoquark for the $\beta=1$ case consists of one isolated high- $p_{T}$ muon, large missing transverse energy, and two energetic jets. The full data sample is reduced by preselecting events according to the criteria described in Section 4.8. Each event is required to have at least one muon of type CMUP or CMX. Therefore we calculate two separate combinations of muon trigger, reconstruction, vertex, and selection efficiencies for CMUP and CMX muon types to obtain the event-type efficiencies:

$$
\begin{aligned}
\epsilon_{C M U P} & =\epsilon_{\text {trig }}^{C M U P} \times \epsilon_{\text {fid }} \times \epsilon_{\text {reco }}^{C M U P} \times \epsilon_{I D}^{C M U P} \times \epsilon_{\text {iso }}^{C M U P} \\
\epsilon_{C M X} & =\epsilon_{\text {trig }}^{C M X} \times \epsilon_{\text {fid }} \times \epsilon_{\text {reco }}^{C M X} \times \epsilon_{I D}^{C M X} \times \epsilon_{\text {iso }}^{C M X}
\end{aligned}
$$


Using the values from Table 4.2, we determine two event-type efficiencies:

\begin{aligned} & \hline Event-type Efficiencies, $\mu q \nu q$ channel \\ & \hline \hline$\epsilon_{C M U P}= 0.695 \pm 0.009 \\ & \epsilon_{C M X}= 0.837 \pm 0.008 \\ &$\hline\end{aligned}

\subsection{Acceptance and kinematical efficiency calculation}

After event preselection, the events are further reduced in order to discriminate the leptoquark signal from the backgrounds discussed in Section 5.3. Because the leptoquark mass is relatively higher than the masses associated with the backgrounds, the leptoquark signal is characterized by large muon, $E_{T}$, and jet momentum as well as large invariant masses. The discriminating cuts are optimized to give the highest limit results. As a result the following cuts are placed:

- Z Veto: If the invariant mass of the tight muon and a second loose muon in the event is in the $Z$-boson mass region $76<M_{\mu \mu} / \mathrm{GeV} / \mathrm{c}^{2}<106$, then the event is rejected. For this cut a loose muon criterion is defined as any muon having $E_{E M}<3 \mathrm{GeV}$, $E_{H A D}<9 \mathrm{GeV}, z 0<60 \mathrm{~cm}$, and fractional isolation $<0.1$.

- Orthogonality Condition: To remain orthogonal to the $\beta=1$ leptoquark decay channel, the selection criteria must be exclusive with respect to the selection criteria of the $\mu q \mu q$ channel analysis. Therefore if an event has a second tight muon as defined in Section 5.1, then it is categorically rejected.

- One muon with $p_{T}(\mu)>25 \mathrm{GeV} / \mathrm{c}$ and $E_{T}>60 \mathrm{GeV}$. This cut selects the muon and the neutrino manifested as $\mathbb{E}_{T}$. The large $\mathbb{E}_{T}$ energy value greatly reduces QCD background.

- Two jets with $E_{T}(j 1)>30 \mathrm{GeV}$ and $E_{T}(j 1)+E_{T}(j 2)>80 \mathrm{GeV}$.

- $\Delta \phi\left(\right.$ leadingjets, $\left.\mathbb{E}_{T}\right)>5^{\circ}$. This topology cut rejects events in which the jets are not isolated from the neutrino.

- $\Delta \phi\left(\mu, E_{T}\right)-\pi>5^{o}$. This topology cut rejects events in which the muon and neutrino are back-to-back. An excess of this type of events is usually due to a mismeasured muon which artificially gives rise to a large $\mathbb{E}_{T}$ value pointing in the direction opposite to the muon.

- $M_{T}\left(\mu, E_{T}\right)>120 \mathrm{GeV} / \mathrm{c}^{2}$. The transverse mass of the muon and neutrino is defined as:

$$
M_{T}=\sqrt{\left(p_{T}(\mu) c+\#_{T}\right)^{2}-\left(\mathbf{P}_{T}^{\mu} c+\mathbf{E}_{\mathbf{T}}\right)^{2}} / c^{2} .
$$


This cut greatly reduces background from $W \rightarrow \mu \nu$ and $Z / \gamma^{*} \rightarrow \mu \mu$ background as shown in Figure 5.1.

- Mass Cut. This is a cut on the reconstructed masses of the combinations $\left(j_{1}, \mu\right)$, $\left(j_{2}, \mu\right),\left(j_{1}, \#_{T}\right)$, and $\left(j_{2}, \#_{T}\right)$, and is discussed in Section 6.2.1.

\subsubsection{Mass Cut}

We require that the reconstructed mass combinations of the jets, muon, and $E_{T}$ be consistent with those reconstructed from the leptoquark Monte Carlo. This cut was adopted from the method used in Run I [83]. The combinatorics of the event topology, namely the ambiguity of the jet assignments, allow for two different sets of reconstructed leptoquark pairs in each event. The method used in this analysis is to construct distributions of the mass shapes from the leptoquark Monte Carlo by matching the reconstructed objects to the generator level objects. The results of fitting the distribution for each leptoquark mass are summarized in Table 6.1. These shapes are then used to make the following selection requirements:

1. $\left|M\left(\mu, j_{1}\right)-M_{L Q}\right|<2 \sigma_{1}$ or $\left|M\left(\mu, j_{2}\right)-M_{L Q}\right|<2 \sigma_{2}$, and

2. $M_{T}\left(\not_{T}, j_{1}\right)>T_{1}^{m i n}$ or $M_{T}\left(\not_{T}, j_{2}\right)>T_{2}^{\min }$,

where $M_{L Q}$ is the mean of the reconstructed leptoquark distribution and $\sigma_{1,2}$ are the width parameterizations. $M\left(\mu, j_{1,2}\right)$ are the reconstructed muon-jet invariant masses while $M_{T}\left(E_{T}, j_{1,2}\right)$ are the reconstructed transverse invariant masses. For the mass reconstruction, full jet energy corrections are used (level $=7$ ). As the leptoquark mass increases, the mass resolution decreases which results in wider mass distributions. For the transverse mass distributions, we have chosen a mass-dependent lower cut denoted by $T_{1,2}^{\min }$ :

- $T_{1}^{m i n}=20+\left(M_{L Q}-120\right) \mathrm{GeV} / \mathrm{c}^{2}$, and

- $T_{2}^{\min }=20+\left(M_{L Q}-120\right) / 2 \mathrm{GeV} / \mathrm{c}^{2}$.

Figure 6.1 shows the signal distributions for a leptoquark mass of $180 \mathrm{GeV} / \mathrm{c}^{2}$. The mass distributions before the mass cut are shown in Figures $6.2-6.3$.

\begin{tabular}{|l|lllllll|}
\hline Mass & 140 & 160 & 180 & 200 & 220 & 240 & 260 \\
\hline$\sigma_{1}\left(\mu, j_{1}\right)$ & 10.31 & 13.63 & 14.65 & 14.46 & 15.72 & 19.31 & 23.68 \\
\hline$\sigma_{2}\left(\mu, j_{2}\right)$ & 14.83 & 18.66 & 19.33 & 20.18 & 26.84 & 27.37 & 27.96 \\
\hline
\end{tabular}

Table 6.1: Mass distribution widths for $\mu$-jet1 and $\mu$-jet2. 

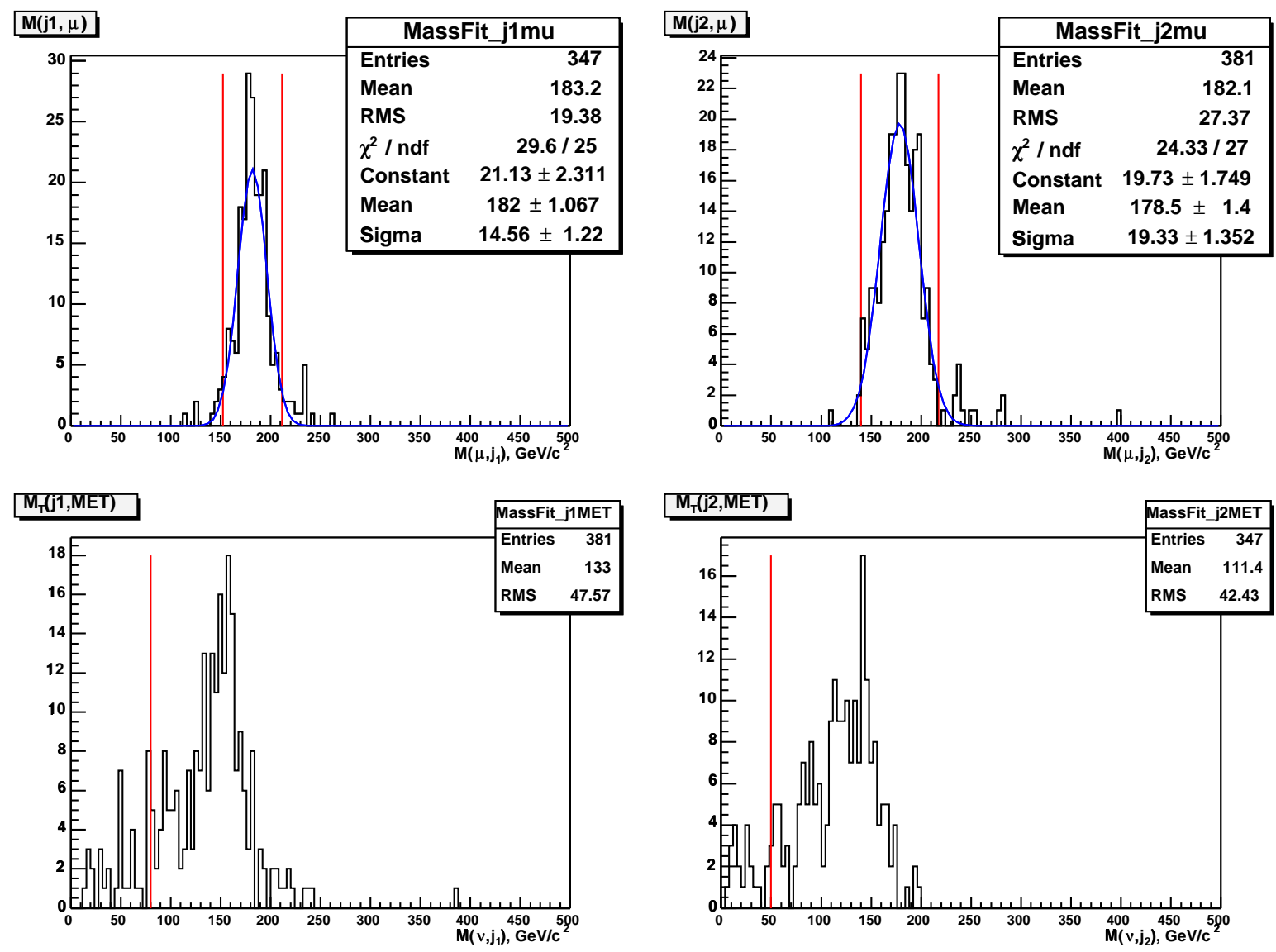

Figure 6.1: Reconstructed mass distributions for $M_{L Q}=180 \mathrm{GeV} / \mathrm{c}^{2}$. The red lines represent the cuts made based on the distribution widths and the generated leptoquark mass.

The mass selection criterion in this analysis is one of several methods of choosing the jet assignments to the muon and $E_{T}$. Two alternative approaches are: 1.) to choose the combination which yeilds the minimum mass difference between the two constructed leptoquark masses, or 2.) to choose the jet combination based on the maximum angle, or back-to-back, distributions of the event topology. The efficiencies for these methods compared to the one chosen for this analysis are shown in Table 6.2.

\subsubsection{Total Acceptances}

The analysis cut efficiencies are calculated relative to the number of events of type CMUP or CMX and are reported in Figure 6.4 and Table 6.3. The analysis cut efficiencies are then folded with the Z-vertex cut (fiducial) [90], trigger [95], muon reconstruction, and 
CHAPTER 6. SEARCH FOR SCALAR LEPTOQUARKS IN THE $\mu Q \nu Q$ CHANNEL 98

\begin{tabular}{ccccc}
\hline Method & $\mathrm{LQ}\left(180 \mathrm{GeV} / \mathrm{c}^{2}\right)$ & $W+\geq 2$ jets & $\ell+$ jets & $S / \sqrt{B}$ \\
\hline 4 Mass Combo & $88 \pm 0 \%$ & $60 \pm 7 \%$ & $51 \pm 1 \%$ & 3.17 \\
$\min (\Delta M)$ & $82 \pm 0 \%$ & $50 \pm 7 \%$ & $66 \pm 1 \%$ & 2.88 \\
$\max (\Delta \phi)$ & $69 \pm 0 \%$ & $90 \pm 9 \%$ & $78 \pm 1 \%$ & 2.06 \\
\hline
\end{tabular}

Table 6.2: The acceptance efficiencies for the various criteria of jet assignment for the leptoquark mass cut.

fractional isolation cut efficiencies. The expected number of signal events in $198 \mathrm{pb}^{-1}$ given the above efficiencies and the NLO theoretical cross section for different values of the renormalization/factorization scale is reported in Table 6.4.

\begin{tabular}{|c|c|c|c|}
\hline $\begin{array}{c}\text { Mass } \\
\left(\mathrm{GeV} / \mathrm{c}^{2}\right)\end{array}$ & CMUP \pm stat \pm sys(\%) & CMX \pm stat \pm sys $(\%)$ & Total \pm stat \pm sys $(\%)$ \\
\hline 140 & $3.99 \pm 0.05 \pm 0.32$ & $1.19 \pm 0.02 \pm 0.11$ & $5.18 \pm 0.07 \pm 0.43$ \\
160 & $5.36 \pm 0.07 \pm 0.41$ & $1.97 \pm 0.03 \pm 0.16$ & $7.33 \pm 0.10 \pm 0.57$ \\
180 & $6.79 \pm 0.09 \pm 0.50$ & $2.66 \pm 0.04 \pm 0.20$ & $9.45 \pm 0.13 \pm 0.70$ \\
200 & $7.73 \pm 0.10 \pm 0.54$ & $3.15 \pm 0.04 \pm 0.22$ & $10.9 \pm 0.14 \pm 0.76$ \\
220 & $9.14 \pm 0.12 \pm 0.62$ & $3.39 \pm 0.05 \pm 0.23$ & $12.5 \pm 0.17 \pm 0.85$ \\
240 & $8.76 \pm 0.11 \pm 0.59$ & $3.57 \pm 0.05 \pm 0.24$ & $12.3 \pm 0.16 \pm 0.83$ \\
260 & $10.2 \pm 0.13 \pm 0.68$ & $4.14 \pm 0.06 \pm 0.28$ & $14.3 \pm 0.19 \pm 0.96$ \\
\hline
\end{tabular}

Table 6.3: Kinematic efficiency as a function of the leptoquark mass.

\subsection{Background calculation in the $\mu q \nu q$ channel}

The main sources of background are due to $W+\geq 2$ jets with $W \rightarrow \mu \nu, t \bar{t}$ production, $\gamma^{*} / Z \rightarrow \mu^{+} \mu^{-}+2 j e t s$, cosmic ray events accompanied by jets due to radiation and events due to $\mathrm{QCD} /$ fakes. Other backgrounds from $b \bar{b}, W \rightarrow \tau \nu, W^{+} W^{-}$are expected to be negligible due to the isolation cut and large muon and jet transverse energy requirements. Table 6.5 summarizes the background estimation in the $\mu q \nu q$ sample.

The following Monte Carlo samples are generated to determine the number of expected background events in the data sample:

- $W+\geq 2$ jets where $W \rightarrow \mu \nu$. This background constitutes the largest contribution of background events. Approximately 200,000 events are processed from the atop08 sample generated by the CDF Top Group using Pythia. A NLO cross section of $294.8 \pm 9.2 \mathrm{pb}$ is calculated using the Monte Carlo parton generator MCFM [70] with a factorization scale of $Q^{2}=M_{W}^{2}$ and PDF set CTEQ4M. 


\begin{tabular}{|cc|cc|}
\hline Mass & $N_{95 \%}$ & \multicolumn{2}{|c|}{ Expected Events $\times \beta$} \\
\cline { 3 - 4 }$\left(\mathrm{GeV} / \mathrm{c}^{2}\right)$ & & $Q^{2}=M_{L Q}^{2} / 4$ & $Q^{2}=4 M_{L Q}^{2}$ \\
\hline 140 & 5.51 & 13.64 & 10.72 \\
160 & 5.20 & 8.63 & 6.88 \\
180 & 4.48 & 5.34 & 4.28 \\
200 & 3.04 & 3.10 & 2.51 \\
220 & 3.04 & 1.87 & 1.51 \\
240 & 3.04 & 0.99 & 0.80 \\
260 & 3.04 & 0.64 & 0.51 \\
\hline
\end{tabular}

Table 6.4: The measured upper limit on the expected number of background subtracted signal events in $198 \mathrm{pb}^{-1}$ for $L Q \overline{L Q} \rightarrow \mu q \nu q$. The right two columns show the theoretically expected number of scalar leptoquark events for different factorization/renormalization scales.

\begin{tabular}{|l|c|c|c|l|}
\hline \multicolumn{5}{|c|}{$M_{L Q}=180 \mathrm{GeV} / \mathrm{c}^{2}$} \\
\hline Process & $\mathrm{N}_{\text {gen }}$ & $\sigma \cdot B R(\mathrm{pb})$ & $\mathrm{N}_{\text {exp }}$ & Comments \\
\hline$W+\geq 2$ jets & $200 \mathrm{k}$ & $294.8 \pm 9.19$ & $1.39 \pm 0.10$ & atop08 \\
\hline$t \bar{t}(\ell+$ jets $)$ & $40 \mathrm{k}$ & $6.70 \pm 0.71$ & $1.27 \pm 0.15$ & ttopli \\
\hline$t \bar{t}($ Dilepton $)$ & $40 \mathrm{k}$ & $6.70 \pm 0.71$ & $0.09 \pm 0.01$ & ttopli \\
\hline DY $Z / \gamma \rightarrow \mu \mu+j j$ & $159 \mathrm{k}$ & $65.5 \pm 1.7$ & $0.00 \pm 0.00$ & $10<M_{\mu \mu}<75$ \\
& $138 \mathrm{k}$ & $31.4 \pm 1.0$ & $0.10 \pm 0.01$ & $75<M_{\mu \mu}<105$ \\
& $30 \mathrm{k}$ & $1.04 \pm 0.03$ & $0.09 \pm 0.01$ & $105<M_{\mu \mu}<800$ \\
\hline QCD/Fakes & - & - & $0.29 \pm 0.29$ & from data \\
\hline \hline Total & - & - & $3.22 \pm 0.34$ & \\
\hline
\end{tabular}

Table 6.5: The background processes considered for the signal contamination. The expected background events column shows values obtained for a leptoquark mass $M_{L Q}=180 \mathrm{GeV} / \mathrm{c}^{2}$.

- Top quark production. Backgrounds from top quark production are possible in two scenarios. First is the lepton + jets decay channel $t \bar{t} \rightarrow \mu+\geq 2$ jets and the second is the dilepton + jets channel $t \bar{t} \rightarrow \mu \mu+\geq 2$ jets where one of the two muons escapes detection or is mismeasured. We used 40,000 top events decaying to all channels from the ttopli Monte Carlo sample generated by the CDF Top Group.

- $Z / \gamma^{*} \rightarrow \mu \mu+\geq 2$ jets. The Drell-Yan +2 jets background where one of the muons is unidentified or mismeasured is studied by generating the process using ALPGEN [81]. Approximately 327,000 events were generated comprising three different ranges of dimuon mass $\left(\mathrm{GeV} / \mathrm{c}^{2}\right): 10<M_{\mu \mu}<75,75<M_{\mu \mu}<105$, and $105<M_{\mu \mu}<800$. For each mass region the cross section was calculated using MCFM and is shown in Table 6.5. 
The probability of "fake" muons coming from energetic jets or pion decay which pass the trigger requirement is negligible due to the muon stub-track match requirement. However QCD multijet backgrounds are still a possible source of signal contamination, even with the high- $E_{T}$ cut. The QCD multijet background is estimated by examining the phase-space of the $E_{T}$ vs the muon fractional isolation for data events in which the muon isolation requirement is not enforced. Figure 6.5 shows the candidate events in the data prior to any kinematic cuts. The QCD multijet events dominate the region $E_{T}<10 \mathrm{GeV}$, while the $W+\geq 2$ jets and $\ell+\geq 2$ jets events dominate the region $10<\boldsymbol{E}_{T} / \mathrm{GeV}<60$.

To estimate the number of QCD events in the signal region $\mathbb{E}_{T}>60 \mathrm{GeV}, I_{0.4} / p_{T}<0.1$, we calculate the number of QCD events in the region $\not_{T}<10 \mathrm{GeV}$ which defines the QCD contamination up to a normalization factor. The estimated number of QCD events in the signal region is estimated as:

$$
N(\text { region I })=\frac{N(\text { region IV }) \times N(\text { region II })}{N(\text { region III })}
$$

where the regions I-IV are defined in Table 6.6. Figure 6.6 shows the events surviving the

\begin{tabular}{ll}
\hline Region & Condition \\
\hline \hline I & $E_{T}>60 \mathrm{GeV}, I_{0.4} / p_{T}<0.1$ \\
II & $Z_{T}>60 \mathrm{GeV}, I_{0.4} / p_{T}>0.2$ \\
III & $E_{T}<10 \mathrm{GeV}, I_{0.4} / p_{T}>0.2$ \\
IV & $E_{T}<10 \mathrm{GeV}, I_{0.4} / p_{T}<0.1$ \\
\hline
\end{tabular}

Table 6.6: QCD background calculation regions.

kinematic cuts before the transverse mass cut. The number of events in each region and the calculated QCD contamination is reported after each kinematic cut in Table 6.7. This

\begin{tabular}{lrrrr}
\hline Kinematic Cut & I & II & III & IV \\
\hline Z,extra $\mu$ veto & 37290 & 391 & 32897 & 14461 \\
$p_{T}(\mu)>25 \mathrm{GeV} / \mathrm{c}$ & 36932 & 379 & 8111 & 4702 \\
2 jets $>30 \mathrm{GeV}$ & 131 & 21 & 233 & 63 \\
Topology Cuts & 61 & 9 & 218 & 54 \\
$\Sigma E_{T}(j e t s)>80 \mathrm{GeV}$ & 52 & 9 & 154 & 39 \\
$M_{T}\left(\mu, \not_{T}\right)>120 \mathrm{GeV} / \mathrm{c}^{2}$ & 4 & 5 & 0 & 0 \\
Mass Cut & 2 & 0 & 0 & 0 \\
\hline
\end{tabular}

Table 6.7: Events surviving the kinematic cuts in the regions defined in Table 6.6. The number of events surviving the mass cut in region I is for $M_{L Q}=180 \mathrm{GeV} / \mathrm{c}^{2}$.

sample is then further reduced by subtracting the contributions of the $W+\geq 2$ jets and $\ell+$ 


\begin{tabular}{cccccc}
\hline & 140 & 160 & 180 & 200 & 220 \\
\hline \hline$W+$ jets & $0.92 \pm 0.06$ & $1.39 \pm 0.09$ & $1.39 \pm 0.10$ & $1.62 \pm 0.11$ & $1.60 \pm 0.11$ \\
$t \bar{t}$ & $1.69 \pm 0.21$ & $1.84 \pm 0.23$ & $1.35 \pm 0.17$ & $1.00 \pm 0.37$ & $0.80 \pm 0.29$ \\
$Z+$ jets & $0.18 \pm 0.01$ & $0.22 \pm 0.02$ & $0.19 \pm 0.01$ & $0.18 \pm 0.01$ & $0.14 \pm 0.01$ \\
QCD multijet & $0.29 \pm 0.29$ & $0.29 \pm 0.29$ & $0.29 \pm 0.29$ & $0.29 \pm 0.29$ & $0.29 \pm 0.29$ \\
Total & $3.09 \pm 0.35$ & $3.74 \pm 0.36$ & $3.22 \pm 0.34$ & $3.08 \pm 0.33$ & $2.83 \pm 0.32$ \\
\hline Data & 3 & 3 & 2 & 0 & 0 \\
\hline
\end{tabular}

Table 6.8: The final events calculated for expected backgrounds and observed events in the data for several scalar leptoquark masses.

jets backgrounds.

The cut on the transverse mass reduces the number of events such that Equation 6.4 is not well behaved. To determine the final number of QCD background events, we calculate the ratio of events in the small $E_{T}$ region III and IV after the cut on $\Sigma E_{T}(j e t s)$. Assuming the ratio is independent of the transverse mass, this normalization factor can then be applied to the number of events in the high- $\not_{T}$ region with large isolation to determine the number of QCD events in the signal region. However, the number of events in Region II surviving the mass cuts for all leptoquark mass contours is zero. We therefore estimate the number of QCD events in the signal region by applying the normalization factor to the error on the mean of the one-sigma Poisson distribution after the cut on transverse mass. We estimate $0.29 \pm 0.29$ QCD events in our data sample after all selection cuts.

Table 6.8 shows the estimated background events and the number of events surviving selection.

\subsection{Cross-check with the $W+\geq 2$ jets process}

In order to check that our acceptances and efficiencies are compatible with other measurements, we check the number of events in the control region $\not_{T}>20 \mathrm{GeV}$ and $p_{T}(\mu)>25$ $\mathrm{GeV} / \mathrm{c}$, and $E_{T}\left(j_{1,2}\right)>30 \mathrm{GeV}$ and check it is consistent with the production of $W+\geq 2$ jets. The number of expected events is calculated from the formula:

$$
\left(N_{W}-N_{B G}\right)=\sigma \times B R(W \rightarrow \mu \nu) \times A(W) \times \epsilon \times \mathscr{L}
$$

where the majority of background events come from the $t \bar{t}$ production with decay mode $\ell+$ jets. Using the values listed in Table 6.9 , we obtain for the $W+\geq 2$ jets background a number of observed events compatible with the NLO calculation. A plot of the transverse mass distribution for the Monte Carlo and the data is shown in Figure 6.7. 


\begin{tabular}{|l|l|l|}
\hline & CMUP & CMX \\
\hline $\mathrm{A}(\mathrm{W}+2$ jets $) \%$ & $0.37 \pm 0.00$ & $0.16 \pm 0.00$ \\
\hline$\epsilon_{\mu \mu}$ & $0.70 \pm 0.01$ & $0.84 \pm 0.01$ \\
\hline$\sigma(W+\geq 2$ jets $) \cdot B R(W \rightarrow \mu \nu), \mathrm{pb}$ & \multicolumn{2}{|c|}{$294.8 \pm 9.19$} \\
\hline $\mathscr{L}, \mathrm{pb}^{-1}$ & $197.9 \pm 11.7$ & $179.2 \pm 10.6$ \\
\hline$N_{\text {bkg }}$ & $84.2 \pm 12.3$ & $42.1 \pm 7.4$ \\
\hline$N_{\text {exp. }}$ & $152.1 \pm 10.3$ & $68.8 \pm 4.6$ \\
\hline \hline$N_{\text {obs. }}-N_{\text {bkg }}$ & $133.8 \pm 27.1$ & $69.9 \pm 15.8$ \\
\hline
\end{tabular}

Table 6.9: Parameters used in the calculation of the expected number of $W+\geq 2$ jet events

\subsection{Systematic uncertainty}

The following systematic uncertainties are considered:

- Luminosity [56]: $6 \%$

- Acceptance

- PDF: $2.1 \%$

- ISR/FSR: $1.8 \%$

- Statistical Error of Monte Carlo: $2.2 \%$

- Jet Energy Scale: $<1 \%$

- Muon ID efficiency $[18,19]$

- muon reconstruction: $0.3 \%$

- Event vertex cut [90]: 0.5\%

Adding the above systematic uncertainties in quadrature will give a total systematic uncertainty of about $7-8 \%$. The statistical, systematic, and combined relative errors are listed in Table 6.10.

\subsection{Cross section limit}

The production cross section $\sigma$ of the process $L Q \overline{L Q} \rightarrow \mu q \nu q$ can be written as follows:

$$
\sigma \times B R(L Q \overline{L Q} \rightarrow \mu q \nu q)=\sigma \times 2 \beta(1-\beta)=N /(\epsilon \times \mathscr{L})
$$

where $N$ is the number of observed data events after our selection, $\epsilon$ is the total selection efficiency as a function of $M_{L Q}$ and $\mathscr{L}$ is the integrated luminosity. The number of candidate 


\begin{tabular}{|l|ccccc|c|}
\hline Mass & $\mathrm{A}\left(M_{L Q}\right) \%$ & \pm & Statistical\% & \pm & Systematic\% & Combined Rel.\% \\
\hline 140 & 5.34 & \pm & 0.07 & \pm & 0.44 & 8.44 \\
160 & 7.57 & \pm & 0.10 & \pm & 0.58 & 7.92 \\
180 & 9.75 & \pm & 0.13 & \pm & 0.72 & 7.61 \\
200 & 11.2 & \pm & 0.2 & \pm & 0.8 & 7.23 \\
220 & 13.0 & \pm & 0.2 & \pm & 0.9 & 7.01 \\
240 & 12.8 & \pm & 0.2 & \pm & 0.9 & 6.93 \\
260 & 14.6 & \pm & 0.2 & \pm & 1.0 & 6.91 \\
\hline
\end{tabular}

Table 6.10: The total acceptance percentages for each leptoquark mass listed with the statistical, systematic and combined relative errors.

events in our leptoquark mass-dependent selections are shown in Table 6.8. We observe no excess of events and thus set a 95\% C.L. upper limit on the cross section as a function of $M_{L Q}$ defined as:

$$
\sigma^{l i m}=\frac{N^{l i m}}{\epsilon_{t o t} \times \mathscr{L}} .
$$

In Table 6.11 we report the $\beta=1 / 2$ values of the cross section limits in $\mu q \nu q$ for each $M_{L Q}$ and the theoretical calculations at NLO for pair production of scalar leptoquarks at the Tevatron using the CTEQ4M PDF with different choices of scale. In Figure 6.8 the

\begin{tabular}{|c|c|c|c|}
\hline Mass & $95 \%$ CL $\sigma(\mathrm{pb})$ & \multicolumn{2}{|c|}{$\sigma \times 0.5$ Theory CTEQ4M $(\mathrm{pb})$} \\
\cline { 3 - 4 }$\left(\mathrm{GeV} / \mathrm{c}^{2}\right)$ & & $Q^{2}=M_{L Q}^{2} / 4$ & $Q^{2}=4 M_{L Q}^{2}$ \\
\hline 140 & 0.538 & 1.330 & 1.045 \\
160 & 0.359 & 0.595 & 0.474 \\
180 & 0.240 & 0.285 & 0.229 \\
200 & 0.141 & 0.144 & 0.117 \\
220 & 0.123 & 0.075 & 0.061 \\
240 & 0.125 & 0.041 & 0.033 \\
260 & 0.107 & 0.022 & 0.018 \\
\hline
\end{tabular}

Table 6.11: Values of the upper limits at $95 \%$ CL of the production cross section of second generation leptoquarks decaying into $\mu q \nu q$ channel as a function of $M_{L Q}$. The last 2 columns on the right report the result of the theoretical calculations at Next-To-Leading order with CTEQ4M for different choices of the scale. The upper limit values have been normalized by a factor of $\beta=1 / 2$.

limit cross section as a function of $M_{L Q}$ is compared with the theoretical expectations for $L Q \overline{L Q} \rightarrow \mu q \nu q$. At the intersection point between experimental and theoretical curves we find the lower limit on $M_{L Q}$ at $175 \mathrm{GeV} / \mathrm{c}^{2}$. 

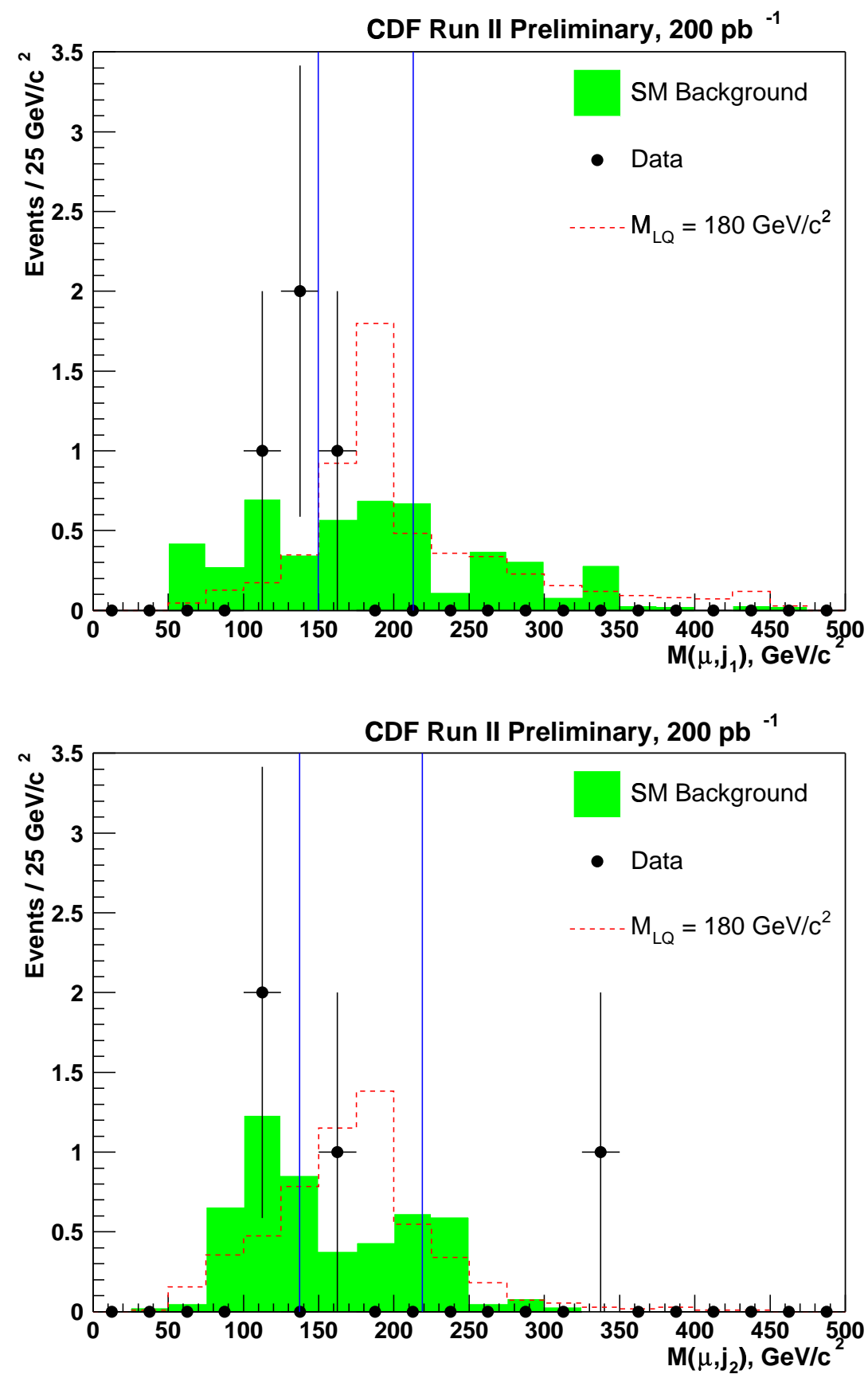

Figure 6.2: The reconstructed mass distributions of the two leading jets and muon for Standard Model background, $M_{L Q}=180 \mathrm{GeV} / \mathrm{c}^{2}$ leptoquark signal, and observed data events. 

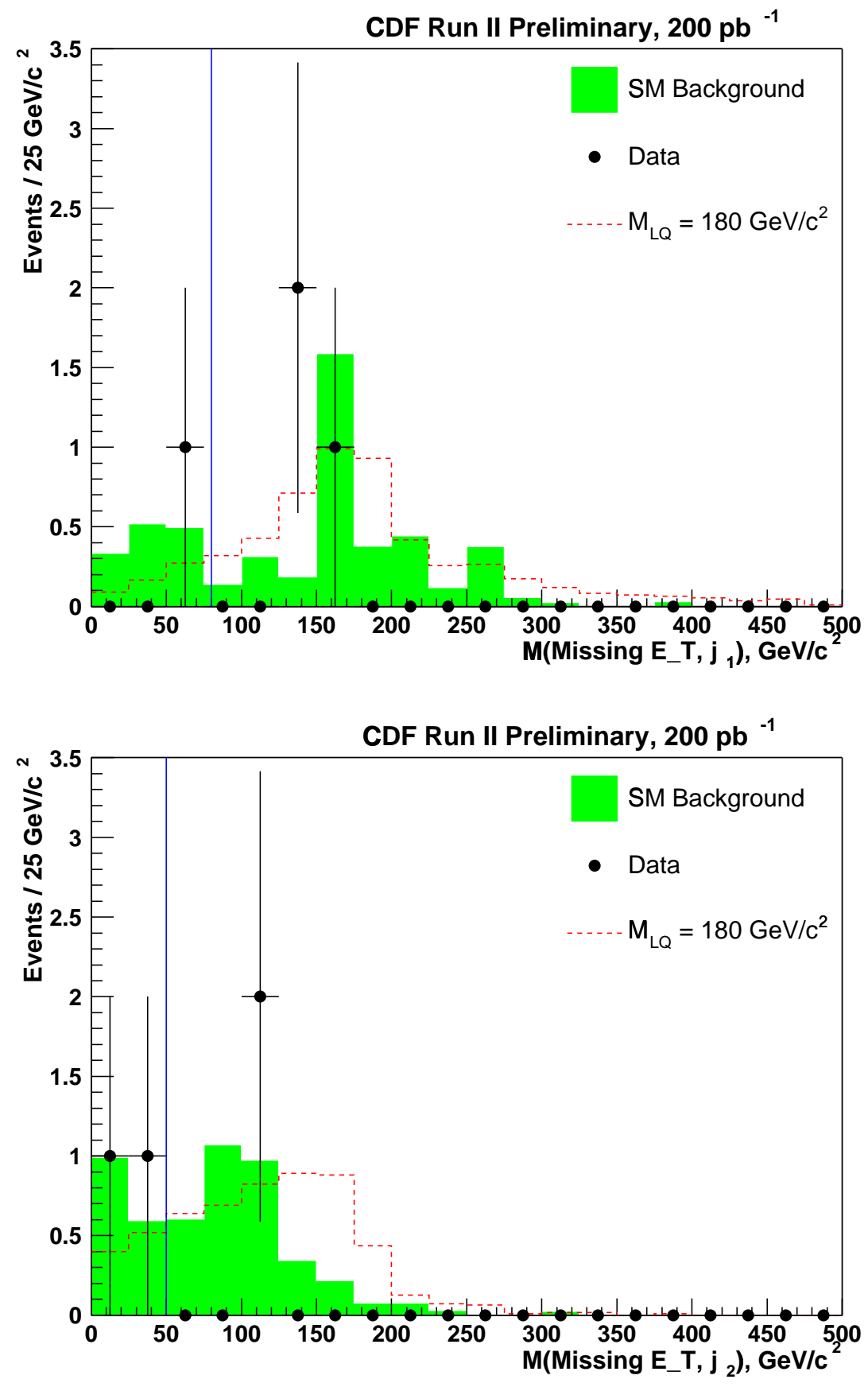

Figure 6.3: The reconstructed transverse mass distributions of the two leading jets and muon for Standard Model background, $M_{L Q}=180 \mathrm{GeV} / \mathrm{c}^{2}$ leptoquark signal, and observed data events. 


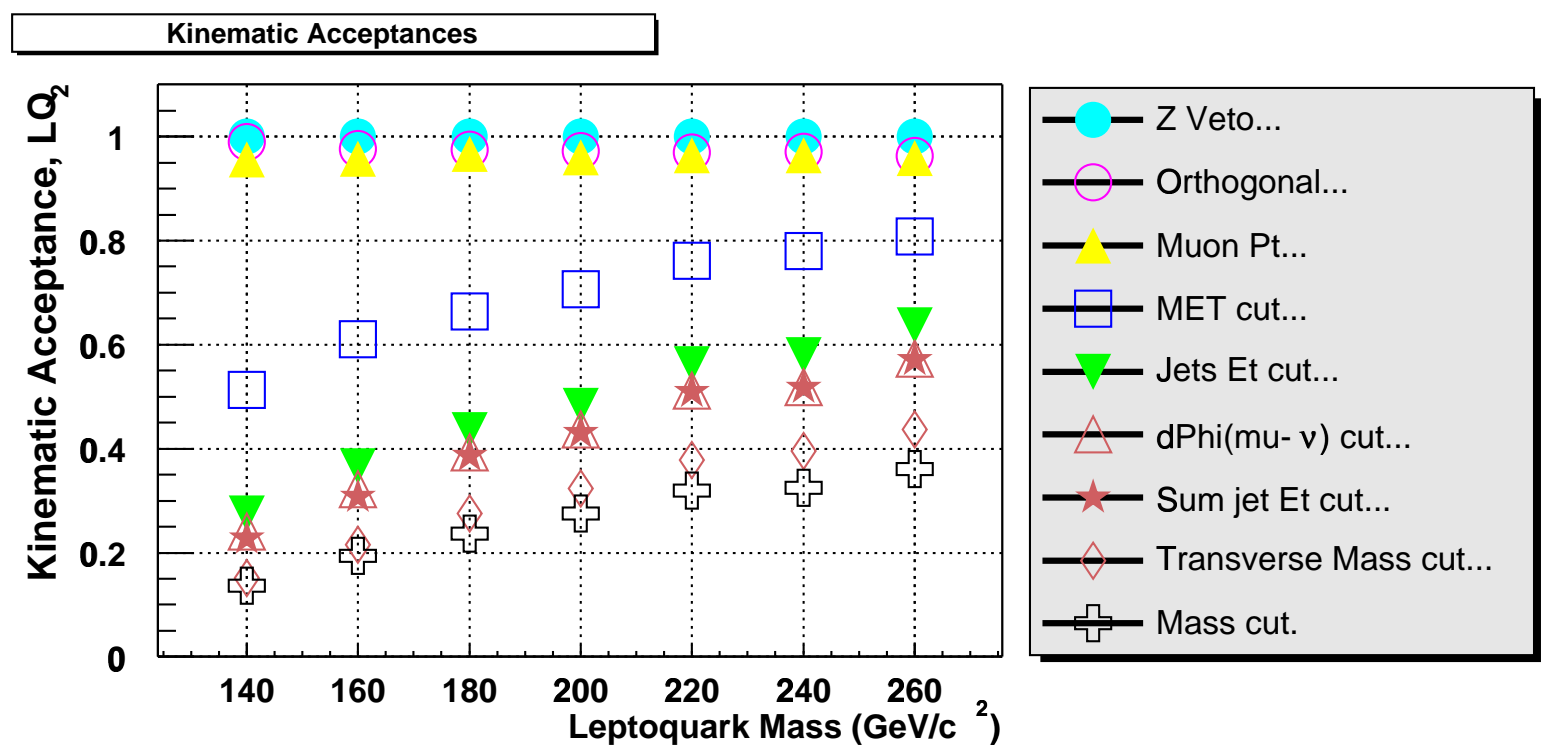

Figure 6.4: Cumulative efficiencies of the kinematic cuts for a range of leptoquark masses. The statistical errors are plotted. 


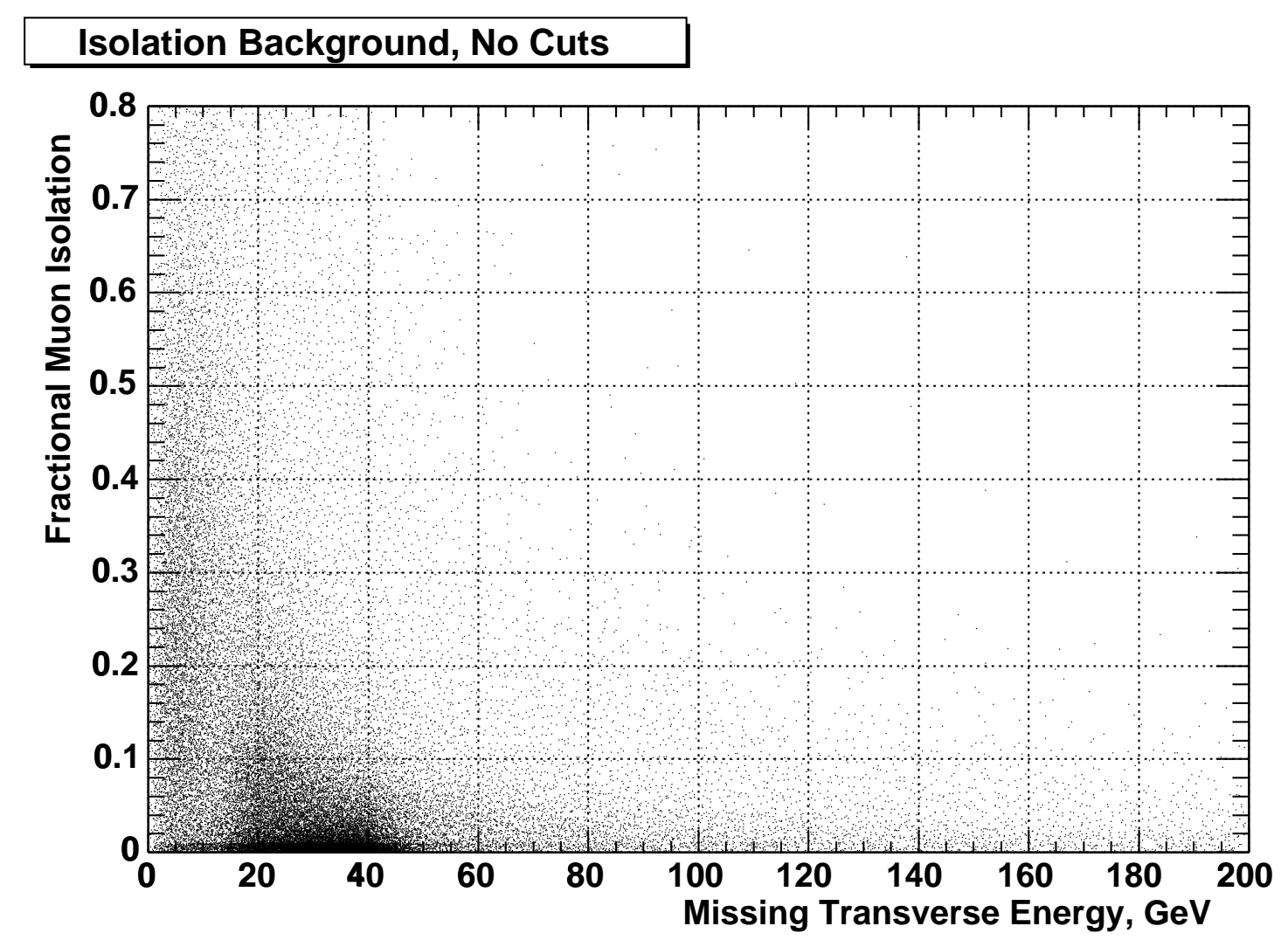

Figure 6.5: The fractional muon isolation versus $\mathbb{E}_{T}$ in $198 \mathrm{pb}^{-1}$ of data before any kinematic cuts. The QCD background dominates the region $\mathbb{F}_{T}<10 \mathrm{GeV}$; the $W+\geq 2$ jets and $\ell+$ jets dominates the region $10<E_{T} / \mathrm{GeV}<60$. 


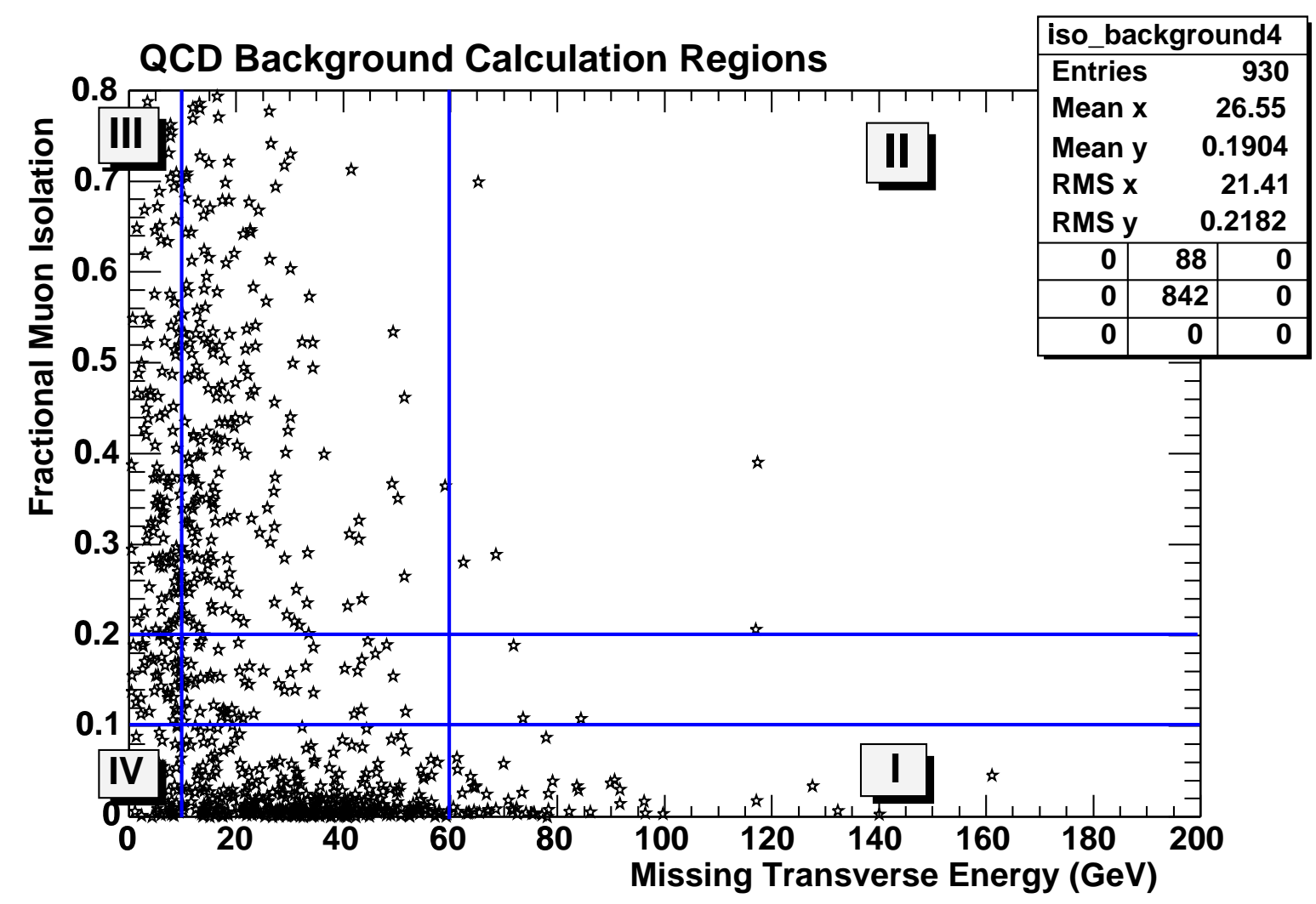

Figure 6.6: The fractional muon isolation versus $\not_{T}$ in $198 \mathrm{pb}^{-1}$ of data before the $M_{T}>120$ $\mathrm{GeV} / \mathrm{c}^{2}$ cut. 


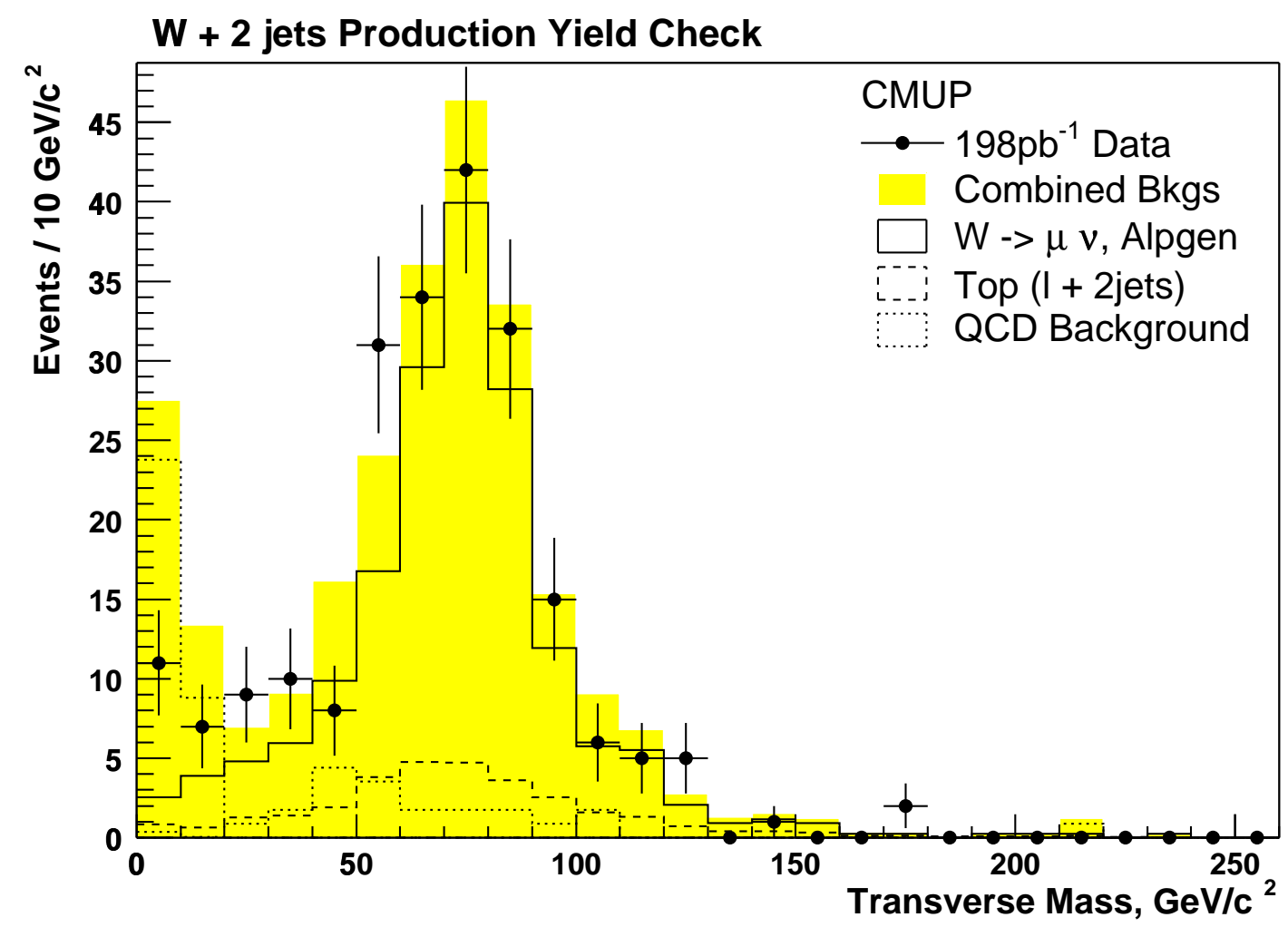

Figure 6.7: Distributions of the invariant transverse mass of the muon and $\mathbb{E}_{T}$ for data (bullets) and Monte Carlo. 


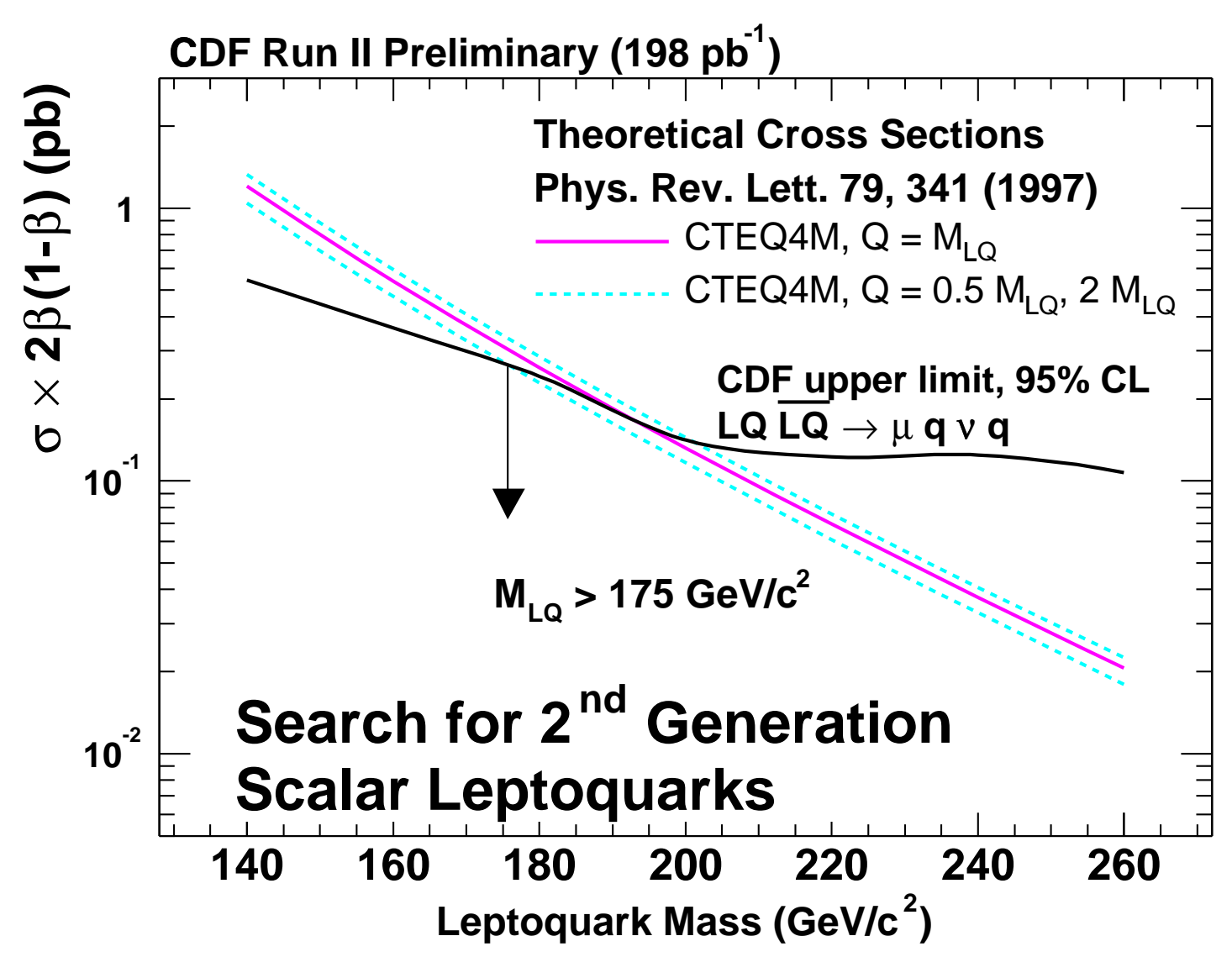

Figure 6.8: Limit cross section as a function of $M_{L Q}$ compared with the theoretical expectations calculated at NLO accuracy. At the intersection points between experimental and theoretical curves we find a lower limit on $M_{L Q}$ at $175 \mathrm{GeV} / \mathrm{c}^{2}$. 


\section{Chapter 7}

\section{Epilogue}

The CDF II experiment began recording $p \bar{p}$ collisions at $\sqrt{s}=1.96 \mathrm{TeV}$ in early 2002 . The most recent results for direct searches of pair-produced leptoquarks predicted to decay into $\mu q \mu q$ and $\mu q \nu q$ using data taken from March 2002 to October 2003, amounting to $198 \mathrm{pb}^{-1}$, have been presented in this dissertation. A paper reporting these results is in preparation for publication.

Leptoquarks are hypothetical color-triplet particles carrying both baryon and lepton quantum numbers and are predicted by many extensions of the Standard Model as new bosons coupling to a lepton-quark pair [1]. Their masses are not predicted. They can be scalar (spin 0 ) or vector (spin 1) particles and at high-energy $p \bar{p}$ colliders such as the Tevatron they would be produced directly in pairs mainly through quark-antiquark annihilation.

The gluon-leptoquark interactions are determined by non-Abelian $S U(3)_{c}$ gauge-symmetry of scalar QCD, making theoretical calculation of the pair-production cross-section independent of the lepton-quark coupling, $\lambda_{l q}$ [1]. Thus for scalar leptoquarks the production crosssection can be calculated as a function of leptoquark mass only. The couplings of vector leptoquarks to the gauge sector are predicted up to model dependent anomalous couplings. The searches reported in this dissertation are for scalar leptoquarks only.

In most models, leptoquarks are expected to couple only to fermions in the same generation because of experimental constraints such as non-observation of flavor changing neutral currents (FCNC) or helicity suppressed decays. Scalar leptoquarks can decay either into a lepton and a quark $(\beta=1)$ or a neutrino and quark $(\beta=0)$, where $\beta$ is the branching ratio of leptoquark decays into a charged lepton-quark pair. The final state decay products of pair produced leptoquarks are $\ell^{ \pm} \ell^{\mp} q \bar{q}, \ell^{ \pm} \nu q \bar{q}$, and $\nu \bar{\nu} q \bar{q}$.

In this analysis the decay signatures studied were $\mu \mu j j$ associated with $\beta=1$ and $\mu \not_{T} j j$ associated with $\beta=1 / 2$ of second-generation scalar leptoquarks. Each analysis selects an exclusive set of candidate leptoquark events and only the central region of the detector is 
utilized. No evidence of scalar leptoquarks is found. Candidate events are selected based on tight kinematic cuts on the transverse energies of the expected decay products of the leptoquark.

In the $\beta=1$ case we select events with two isolated muons with $p_{T}(\mu)>25 \mathrm{GeV} / \mathrm{c}$, one isolated jet with $E_{T}$ (loose) $>15 \mathrm{GeV}$ and one with $E_{T}$ (tight) $>30 \mathrm{GeV}$. The cut $\sqrt{\left(E_{T}\left(j_{1}\right)+E_{T}\left(j_{2}\right)\right)^{2}+\left(p_{T}\left(\mu_{1}\right)+p_{T}\left(\mu_{2}\right)\right)^{2} c^{2}}>200$ is the most discriminating selection criterion in this channel and represents a circular cut centered at the origin in the phase space of the sums of the momentums of the first two leading muons verses the energies of the first two leading jets. This cut eliminates background contributions from Drell-Yan + $\geq 2$ jets and top production. We observe 2 signal events and expect $2.88 \pm 1.14$ events from background processes.

In the $\beta=1 / 2$ case we select events with one muon with $p_{T}>25 \mathrm{GeV} / \mathrm{c}, \not_{T}>60 \mathrm{GeV}$ and two jets each having $E_{T}>30 \mathrm{GeV}$. We also cut on the topology of the event which removes events in which either jet is aligned with the $\mathbb{E}_{T}$ or the muon is anti-aligned with the $\mathbb{E}_{T}$. The most discriminating criterion is made by cuting on the invariant transverse mass of the muon and $\not_{T}$. The final criterion in selecting the candidate leptoquark events is a mass dependent cut on the reconstructed leptoquark mass. No excess of events is observed in comparison with the expected background contributions (see Table 6.8).

For both channels, it has been possible to determine the $95 \%$ CL upper limit on the scalar leptoquark cross section by using a Bayesian method to calculate the upper limit on the number of observed leptoquark events. Comparing the upper limit cross section with the NLO calculation for pair-produced scalar leptoquarks [1], we exclude the existence of second-generation scalar leptoquarks with $M_{L Q_{2}}<221(175) \mathrm{GeV} / \mathrm{c}^{2}$ for $\beta=1(1 / 2)$ at the $95 \%$ CL. Table 7.1 shows the mass limits set on leptoquarks via direct searches in the $\mu q \mu q$ and $\mu q \nu q$ channels with the results from this thesis included in bold.

At the time of this writing, the $\beta=1$ exclusion limit given in this dissertation is the strongest second-generation scalar leptoquark limit set for this channel to date. The previous single channel searches in Run I by CDF resulted in upper limits at $M_{L Q_{2}}>202(164)$ $\mathrm{GeV} / \mathrm{c}^{2}$ for $\beta=1(1 / 2)$. The increase in the limit result of the analyses presented in this dissertation with respect to Run I is consistent with the results obtained in Run I, although a direct comparison is not possible. In addition to the increase in the scalar leptoquark cross section (30\%), the increase of energy results in different background contributions. Also, in the Run I $\beta=1 / 2$ analysis, events were counted from the $\beta=1$ channel in the signal acceptance which inflated the limit result. In the near future we plan to combine the results from the $\beta=1$ and $\beta=1 / 2$ limits set in this dissertation, and the $\beta=0$ results from [24] to obtain a stronger mass limit.

Data continues to be taken at the time of this writing and the integrated luminosity of the 


\begin{tabular}{lll}
\hline Luminosity & \multicolumn{1}{c}{ Mass } & Comments \\
\hline$\mu \mu q q(\beta=1)$ & & \\
$110 \mathrm{pb}^{-1}$ & $202 \mathrm{GeV} / \mathrm{c}^{2}$ & single channel, CDF Collaboration [38] \\
$198 \mathrm{pb}^{-1}$ & $\mathbf{2 2 1} \mathrm{GeV} / \mathrm{c}^{2}$ & single channel, CDF Run II Preliminary \\
$109 \mathrm{pb}^{-1}$ & $209 \mathrm{GeV} / \mathrm{c}^{2}$ & combined result, CDF Run I Preliminary [82] \\
$94 \mathrm{pb}^{-1}$ & $200 \mathrm{GeV} / \mathrm{c}^{2}$ & combined result, DØ Collaboration [48] \\
\hline$\mu \nu q q(\beta=1 / 2)$ & & \\
$94 \mathrm{pb}^{-1}$ & $160 \mathrm{GeV} / \mathrm{c}^{2}$ & single channel, D Ø Collaboration [49] \\
$109 \mathrm{pb}^{-1}$ & $164 \mathrm{GeV} / \mathrm{c}^{2}$ & single channel, CDF Run I Preliminary [83] \\
$198 \mathrm{pb}^{-1}$ & $\mathbf{1 7 5} \mathrm{GeV} / \mathrm{c}^{2}$ & single channel, CDF Run II Preliminary \\
$94 \mathrm{pb}^{-1}$ & $180 \mathrm{GeV} / \mathrm{c}^{2}$ & combined result, DØ Collaboration [48] \\
$109 \mathrm{pb}^{-1}$ & $183 \mathrm{GeV} / \mathrm{c}^{2}$ & combined result, CDF Run I Preliminary [82] \\
\hline
\end{tabular}

Table 7.1: Values of the 95\% CL lower limits on the second generation scalar leptoquark masses from CDF and $\mathrm{D} \varnothing$ experiments. The mass limits in bold denote the results produced in this analysis. The combined results means that the limit was determined by combining results of two or more $\beta$ values.

data sample used in this dissertation will be increased in future leptoquark analyses and other exotics searches at the Tevatron. At the present time, the design goal for delivered luminosity by the end of fiscal year 2009 is approximately $8 \mathrm{fb}^{-1}$. Based on the leptoquark signal acceptance calculated in this dissertation for a leptoquark having mass $M_{L Q_{2}}=320(260)$ $\mathrm{GeV} / \mathrm{c}^{2}$, the sensitivity of this analyses using this amount of data is about $300(270) \mathrm{GeV} / \mathrm{c}^{2}$ for $\beta=1(1 / 2)$.

In addition to the Tevatron, other high energy colliders are capable of searching for leptoquarks. In $e^{+} e^{-}$collisions such as those produced at the LEP (Linear Electron Positron) Collider, first-generation leptoquarks could be produced through the virtual exchange of quarks in the $t$-channel. Additionally, all three generations of leptoquarks could be produced thrugh $s$-channel $\gamma$ and $Z^{0}$ exchange. For these production modes, the scalar leptoquark production cross section is dependent on the assumed leptoquark Yukawa coupling $\lambda_{\ell q}$. Resonances were searched for in $e^{+} e^{-}$data taken up to 2000 by the ALEPH, DELPHI, L3, and OPAL experiments which lead to limits dependent on the leptoquark coupling. At HERA, an $e p$ collider at DESY, leptoquarks can be resonantly produced by the fusion of an $e$ beam particle with the quark in a proton. Again the mass limit results are dependent on the unknown leptoquark coupling strength. Using data taken from 1994 to 2000 with $\sqrt{s}=300$ and $318 \mathrm{GeV}$, the $\mathrm{H} 1$ collaboration at DESY has ruled out leptoquarks having a mass less than $290 \mathrm{GeV} / \mathrm{c}^{2}$ for $\lambda_{e u}=0.1$, exceeding the results set at LEP [104].

With $8 \mathrm{fb}^{-1}$ of data, the sensitivity of the $\beta=1$ analysis of this dissertation is comparable to the first-generation leptoquark limits set by the ZEUS collaboration with leptoquark 
coupling $\lambda_{e q}=0.5$ [104] (see Figure 2.12). After upgrades in 2000, HERA continues to produce $e p$ collisions and ZEUS and $\mathrm{H} 1$ are projected to accumulate $1 \mathrm{fb}^{-1}$ by the end of fiscal year 2006 [12]. The sensitivity at HERA for $\lambda_{e u}=0.05$ with $800 \mathrm{pb}^{-1}$ is expected to be about $300 \mathrm{GeV} / \mathrm{c}^{2}$ [13]. The leptoquark production cross section depends quadratically on the leptoquark coupling and thus the sensitivity to possible leptoquark production at LEP and HERA decreases significantly with the coupling. Since the leptoquark production at hadronic colliders is independent of the leptoquark coupling, a large portion of phase space remains uniquely explorable by the Tevatron for leptoquark couplings weaker than the electromagnetic scale.

In addition to the new data being taken at the Tevatron, a $\sqrt{s}=14 \mathrm{TeV} p p$ collider called the Large Hadron Collider (LHC) is currently under construction and is scheduled to start collisions in 2007. Leptoquark backgrounds and the NLO scalar leptoquark cross section have been calculated for $p p$ interactions with $\sqrt{s}=14 \mathrm{TeV}[31,14]$ [15]. In contrast to the Tevatron, the scalar leptoquark production process at the LHC is expected to be dominated by gluon fusion. However, the topology of the events are expected to be similar to those at the Tevatron. Once running, the LHC is designed to deliver about $100(30) \mathrm{fb}^{-1}$ of data each year at high(low) performance [16]. After three years at low performance, or one year at high performance, the ATLAS collaboration predicts a sensitivity on first-generation scalar leptoquarks at $M_{L Q_{1}}=1.5 \mathrm{TeV} / \mathrm{c}^{2}$. 


\section{Appendix A}

\section{Muon ID and Isolation Efficiencies}

In this section, we verify the validity of using the beam-constrained COT muon ID and isolation efficiencies by following the 1-leg based or "tight-loose" method described in [18].

The method looks for dimuon events with $80<M_{\mu \mu} / \mathrm{GeV} / \mathrm{c}^{2}<100$ containing same type (CMUP-CMUP, CMX-CMX) and mixed type (CMUP-CMX, CMUP-TRK, CMX-TRK) events. For this study only the $179 \mathrm{pb}^{-1}$ of CMX included events is used. The efficiency is determined by starting with a base sample of events, $R_{t o t}$, to which the ID or isolation cuts are not applied. In these events a tight muon (1st leg) is selected based on the $i$ number of ID or isolation cuts. A loose muon (2nd leg) is then searched for by applying the $i$ cuts and seeing whether it passes or fails. To determine the event yield per cut $R_{i}$, the set of events are selected by requiring that all cuts other than the $i$ 'th cut are passed, i.e.

$$
R_{i}=\epsilon-i \cdot\left(2-\epsilon_{i}\right) \cdot \prod_{j} \epsilon_{j}^{2} \cdot R_{t o t}, \quad(j \neq i)
$$

in which $R_{i}$ contains all possible combinations of tight $(\mathrm{T})$ and loose $(\mathrm{L})$ muons. To determined the total event yield, $R_{0}$, we require that all cuts are applied and all cuts are passed by both muons:

$$
R_{0}=\prod_{i} \epsilon_{i}^{2} \cdot R_{t o t}
$$

Using Equations A.1 A.2, one can solve for the individual efficiencies, $\epsilon_{i}$. They are related to the ratio of the event yield per cut and the total event yield by:

$$
\epsilon_{i}=\frac{2 \times R}{1+R},
$$


where $R \equiv R_{0} / R_{i}$. The errors on the efficiencies are calculated via their moments:

$$
\sigma_{\epsilon}^{2}=\left(\frac{\delta \epsilon}{\delta R}\right)^{2} \cdot \sigma_{R}^{2}
$$

Solving for (A.4) we find:

$$
\sigma_{\epsilon}=\frac{\epsilon \cdot R}{(1+R)} \sqrt{\frac{(1-R)}{R_{0}}}
$$

For the mixed muon-type events (CMUP-CMX, CMUP-TRK, CMX-TRK), the event yields are slightly different to account for a $\mathrm{T}$ or $\mathrm{L}$ second leg of different type than the first T leg:

$$
\begin{aligned}
R_{0} & =\prod_{i} \epsilon_{i} \cdot R_{t o t} \\
R_{i} & =\left(\epsilon_{i}+\left(1-\epsilon_{i}\right)\right) \cdot \prod_{j} \epsilon_{j} \cdot R_{t o t}, \quad(j \neq i) .
\end{aligned}
$$

In this case the ratio $R=R_{0} / R_{i}=\epsilon(1 \mathrm{st} \mathrm{Leg})$ and the efficiencies can be calculated right away. The errors are determined from the binomial error on $R$. Table A.1 lists the efficiencies for each cut for both same and mixed-type dimuon events. The muon isolation and ID efficiencies are calculated separately. A total efficiency for muon ID and isolation is determined by combining the same and mixed-type efficiencies in quadrature, i.e. they are treated as separate measurements.

The results shown in Table A.1 are in close agreement with [80]. The COT hit requirements were enforced in this analysis in consideration of the TRK muons, and were folded into its ID efficiency. 


\begin{tabular}{|l|c|c|c|}
\hline SAME TYPE & CMUP & CMX & TRK \\
\hline $16(\mathrm{ST}), 20(\mathrm{AX})$ & $1.000 \pm 0.000$ & $1.000 \pm 0.000$ & \\
$E_{H A D}<6 \mathrm{GeV}$ & $0.984 \pm 0.003$ & $0.984 \pm 0.005$ & \\
$E_{E M}<2 \mathrm{GeV}$ & $0.963 \pm 0.005$ & $0.977 \pm 0.005$ & \\
$\mathrm{dx}(\mathrm{CMU})<3 \mathrm{~cm}$ & $0.950 \pm 0.005$ & - & \\
$\mathrm{dx}(\mathrm{CMP})<5 \mathrm{~cm}$ & $0.979 \pm 0.004$ & - & \\
$\mathrm{dx}(\mathrm{CMX})<6 \mathrm{~cm}$ & - & $0.971 \pm 0.007$ & \\
\hline $\mathrm{ID}$ eff & $0.882 \pm 0.007$ & $0.933 \pm 0.010$ & \\
\hline $\mathrm{ISO}$ eff & $0.988 \pm 0.003$ & $0.979 \pm 0.006$ & \\
\hline \hline $\mathrm{MIXED} \mathrm{TYPE}$ & \multicolumn{3}{|c|}{} \\
\hline $16(\mathrm{ST}), 20(\mathrm{AX})$ & $1.000 \pm 0.001$ & $1.000 \pm 0.001$ & $0.988 \pm 0.000$ \\
$E_{H A D}<6 \mathrm{GeV}$ & $0.987 \pm 0.001$ & $0.979 \pm 0.002$ & $0.981 \pm 0.000$ \\
$E_{E M}<2 \mathrm{GeV}$ & $0.966 \pm 0.001$ & $0.979 \pm 0.002$ & $0.970 \pm 0.000$ \\
$\mathrm{dx}(\mathrm{CMU})<3 \mathrm{~cm}$ & $0.963 \pm 0.001$ & - & \\
$\mathrm{dx}(\mathrm{CMP})<5 \mathrm{~cm}$ & $0.976 \pm 0.001$ & - & \\
$\mathrm{dx}(\mathrm{CMX})<6 \mathrm{~cm}$ & - & $0.971 \pm 0.001$ & \\
\hline $\mathrm{ID}$ eff & $0.895 \pm 0.001$ & $0.930 \pm 0.001$ & $0.940 \pm 0.000$ \\
\hline ISO eff & $0.976 \pm 0.000$ & $0.976 \pm 0.006$ & $0.978 \pm 0.000$ \\
\hline \hline combined ID eff & $0.888 \pm 0.007$ & $0.932 \pm 0.010$ & $0.940 \pm 0.000$ \\
combined Iso eff & $0.982 \pm 0.003$ & $0.977 \pm 0.006$ & $0.957 \pm 0.000$ \\
\hline
\end{tabular}

Table A.1: Efficiencies obtained from the 1-leg method using $179 \mathrm{pb}^{-1}$ of data. The EM and HAD slide with the muon $P_{T}$ as described in Table 4.2 . 


\section{Appendix B}

\section{ID and Isolation Dependence}

In this section, we check the muon ID efficiency as a function of isolation fraction. The events have been chosen in the same way as the efficiency study described in Section A. The muon ID distribution is shown in Figure B.1. Since we apply the muon ID efficiency factor to the leptoquark acceptance, we wish to check whether this convolution has a significant effect in the Monte Carlo samples. In the discrete case, this is done by weighting the muon ID efficiency relative to the number of muons passing the isolation cuts with a specific fractional isolation, i.e.:

$$
<\epsilon_{i s o}>=\sum_{j}^{M(L Q)} \sum_{i}^{n o . b i n s} \frac{\epsilon_{i s o}(i) \times N(i)}{N_{T O T}}
$$

For this study, we chose a binning of $i=5$ and the deconvoluted efficiency was averaged over the $j=9$ LQ samples. Figure B.2 shows the normalized isolation distributions for CMUP, CMX and TRK for a LQ mass of $240 \mathrm{GeV} / \mathrm{c}^{2}$. The deconvoluted muon ID efficiencies along with the scale factor are listed in Table B.1.

\begin{tabular}{|r|c|c|c|}
\hline Muon Type & $\left\langle\epsilon_{I D}^{\text {deconv }}\right\rangle$ & $\epsilon_{I D}^{D A T A}$ & Scale Factor \\
\hline CMUP & 0.880 & $0.889 \pm 0.006$ & 0.9898 \\
CMX & 0.935 & $0.952 \pm 0.005$ & 0.9821 \\
TRK & 0.950 & $0.929 \pm 0.000$ & 1.0226 \\
\hline
\end{tabular}

Table B.1: The muon ID efficiencies deconvoluted from the muon isolation. 


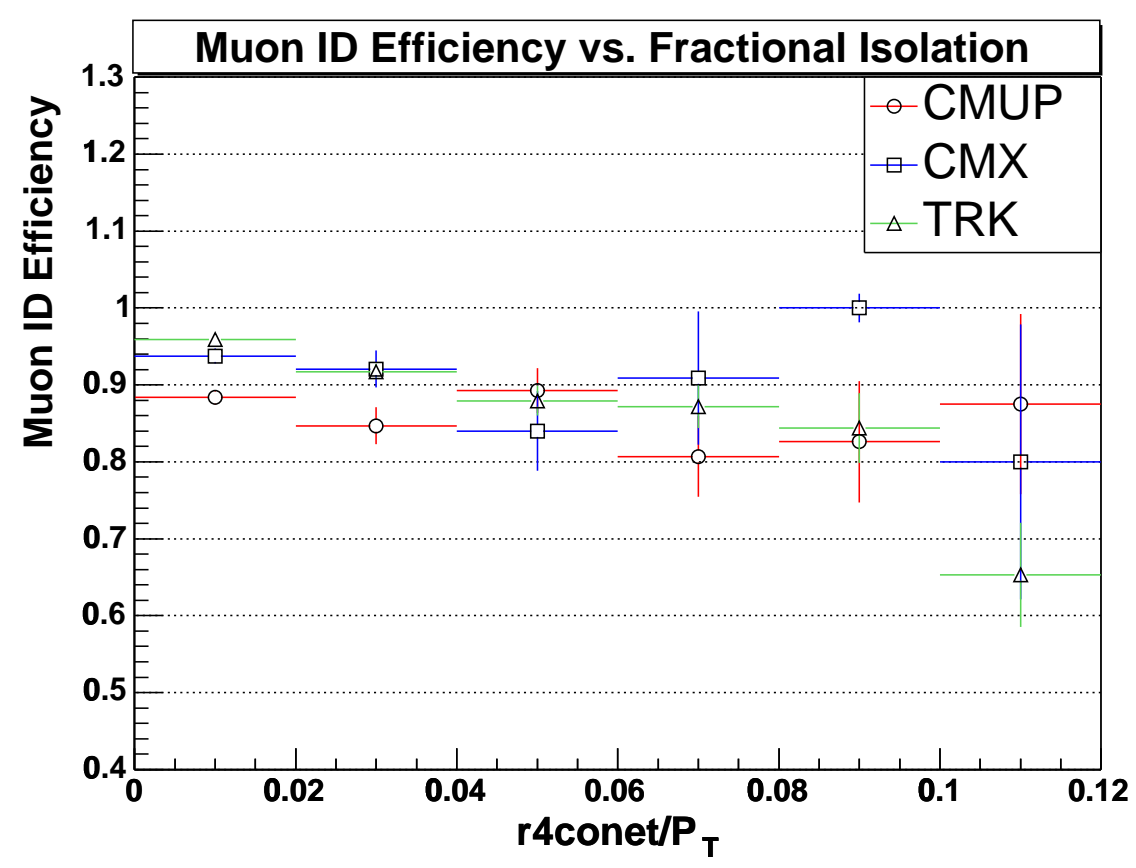

Figure B.1: The muon ID efficiency versus the muon fractional isolation. Each event is required to have at lease one tight muon.

\section{B.1 The Higgs Mechanism}

The Higgs Mechanism is the means by which the particles appearing in the Standard Model Lagrangian acquire a mass and is one if its distinctive features. The Higgs mechanism is achieved by the spontaneous symmetry breaking of the vacuum expectation value. The discrete case of symmetry breaking can be seen in the example of a scalar field with a $\phi^{4}$ interaction:

$$
\begin{aligned}
\mathcal{L} & =\frac{1}{2} \partial_{\mu} \phi \partial^{\mu} \phi-V(\phi), \text { where } \\
V(\phi) & =\frac{1}{2} \mu^{2} \phi^{2}-\frac{1}{4 !} \lambda \phi^{4}
\end{aligned}
$$

and $\lambda$ is a dimensionless constant representing the coupling of the 4-boson vertex. The Lagrangian is symmetric in $\phi=-\phi$, and the potential has 3 possible minimums. If $\mu^{2}>0$, then the local minimum is $\phi_{\text {min }}=0$. However, if $\mu^{2}<0$, then $\phi_{\min }= \pm \sqrt{-\frac{\lambda}{6} \mu^{2}}= \pm \nu$. Since we are concerned with evaluating small perturbations about the energy minimum, we prefer to expand about one of these new minimum, $\phi= \pm \nu$. Normally, we require the vacuum expectation of the scalar field to be zero. However, we have instead, $\langle\phi\rangle_{0}=\nu$. 
This is easily corrected by shifting the value of the scalar field by $\nu$. The new scalar field is given by, $\tilde{\phi}=\nu+h(x)$, where $h(x)$ is the value of the scalar field over and above the constant, uniform value $\nu$. This shifted scalar field gives $\langle\tilde{\phi}\rangle_{0}=0$. Substituting this into our Lagrangian (B.3) gives:

$$
\mathcal{L}=\frac{1}{2}\left(\partial_{\mu} h\right)^{2}+\frac{1}{2} V^{(2)}(\nu) h^{2}+\frac{1}{3 !} V^{(3)}(\nu) h^{3}+\frac{1}{4 !} V^{(4)}(\nu) h^{4} \ldots
$$

where $V^{(2)}(\nu)=\mu^{2}-\frac{1}{2} \lambda \nu^{2}=-2 \mu^{2}, V^{(3)}(\nu)=-\lambda \nu$, and $V^{(4)}(\nu)=-\lambda$. So finally we get:

$$
\mathcal{L}=\frac{1}{2}\left(\partial_{\mu} h\right)^{2}-\frac{1}{2}\left(2 \mu^{2}\right) h^{2}-\frac{1}{3 !} \lambda \nu h^{3}-\frac{1}{4 !} \lambda h^{4} .
$$

The last 2 terms of (B.5) are the same for either value of $\nu$. The first 2 terms are the Lagrangian for a free particle with a mass given by $m_{h}^{2}=2 \mu^{2}$. Since $\mu^{2}<0$, then $m_{h}=$ $\sqrt{-2 \mu^{2}}$ and the mass is positive. Notice that the symmetry $\phi=-\phi$ has been broken once the field shifts to either $\pm \nu$ (see for example [75] [85]).

The continuous case of symmetry breaking can be seen in the example of an $O(2)$ scalar field theory with a $\phi^{4}$ interaction:

$$
\mathscr{L}=\frac{1}{2} \partial_{\mu} \phi^{\dagger} \partial^{\mu} \phi-\frac{\lambda}{4 !}\left(\phi^{\dagger} \phi-\nu^{2}\right)^{2}
$$

and the scalar field now has two components:

$$
\phi=\left(\begin{array}{c}
\phi_{1} \\
\phi_{2}
\end{array}\right) .
$$

Equation B.6 has a circle of degenerate minima at $\left|\phi_{\min }\right|=\nu$. In order to expand about a particular ground state, we prefer to expand about this minimum. This minimum is degenerate, so we are free to choose:

$$
\langle\phi\rangle_{0}=\left(\begin{array}{l}
0 \\
\nu
\end{array}\right) .
$$

Normally, one requires the vacuum expectation value of a scalar field to be zero. This is easily corrected by shifting the value of the scalar field by $\nu$ :

$$
\tilde{\phi}=e^{i \theta(x)}\left(\begin{array}{c}
0 \\
\nu+h(x)
\end{array}\right) .
$$

This new vacuum state is now invariant only under the group which leaves the shifted field invariant. In general, when a field is shifted, the symmetry is broken from $\mathrm{O}(\mathrm{N})$ down to 
$\mathrm{O}(\mathrm{N}-1)$. In the current case, there are only 2 components to $\phi$, so we have broken the symmetry group $\mathrm{O}(2)$ to $\mathrm{O}(1)$.

Substituting the new field (B.9) into our Lagrangian results in :

$$
\mathscr{L}=(\partial h)^{2}+\nu^{2}(\partial \theta)^{2}+2 \nu h(\partial \theta)^{2}+h^{2}(\partial \theta)^{2}-\frac{\lambda}{6} \nu^{2} h^{2}-\frac{\lambda}{6} \nu h^{3}-\frac{\lambda}{4 !} h^{4} .
$$

By making the substitutions $h=1 / \sqrt{2} \phi_{A}, \nu \theta=1 / \sqrt{2} \phi_{B}$, and $m=\sqrt{\lambda / 6} \nu$, Equation (B.10) becomes:

$$
\mathscr{L}=\frac{1}{2}\left(\partial_{\mu} \phi_{A} \partial^{\mu} \phi_{A}\right)-\frac{1}{2} m^{2} \phi_{A}^{2}+\frac{1}{2}\left(\partial_{\mu} \phi_{B} \partial^{\mu} \phi_{B}\right)+\mathscr{L}_{i n t}
$$

where $\mathscr{L}_{\text {int }}$ are the 3 and 4 point interactions between the fields $\phi_{A}$ and $\phi_{B}$. The first two terms in (B.11) comprise the Lagrangian for a massive scalar field $\phi_{A}$, and the second term a free Lagrangian for the field $\phi_{B}$ which apparently has no mass. In addition to the breaking of symmetry of Equation (B.6) by choosing a particular vacuum state, one of the fields automatically becomes massless. Such a massless scalar field is known as a "Goldstone Boson". It can be shown vigorously that for each broken generator of a symmetry group, there will correspond a creation of a massless scalar particle. Breaking the symmetry of a theory from $O(N)$ to $O(N-1)$ will result in $N-1$ Goldstone Bosons. For the case at hand, we broke the $\mathrm{O}(2)$ symmetry of (B.6) which resulted in a Lagrangian which is still symmetric under global $\mathrm{O}(1)$ but now has a massless scalar boson.

Returning to (B.6), we see that the Lagrangian is globally phase invariant. As discussed, this symmetry is not broken under the spontaneous symmetry breaking. Equation (B.6) can be made locally phase invariant by replacing the derivatives with a covariant one (1.2), and giving the new gauge field a free term, i.e. $\partial_{\mu} \rightarrow \mathcal{D}_{\mu}=\partial_{\mu}+i e A_{\mu}$, where $A_{\mu} \rightarrow A_{\mu}-\frac{1}{e} \partial_{\mu} \alpha(x)$ under $\phi \rightarrow e^{i \alpha(x)} \phi$. Equation (B.6) will then become,

$$
\mathscr{L}=\frac{1}{2} \mathcal{D}_{\mu} \phi^{\dagger} \mathcal{D}^{\mu} \phi+\frac{\lambda}{4 !}\left(\phi^{\dagger} \phi-\nu^{2}\right)^{2}-\frac{1}{4} F^{\mu \nu} F_{\mu \nu}
$$

which is locally $U(1)$ gauge invariant. Now we break the symmetry by shifting the field: $\phi \rightarrow \phi=\nu+h(x)$. Inserting into (B.12) by parts (and dropping the dependence on $x$ ), we find,

$$
\begin{aligned}
\mathcal{D}_{\mu} & =\partial_{\mu} h-i e(\nu h) A_{\mu} \\
\mathcal{D}_{\mu} \phi^{\dagger} \mathcal{D}^{\mu} \phi & =\left(\partial_{\mu} h\right)^{2}+e^{2}(\nu+h)^{2} A_{\mu} A^{\mu} \\
\frac{\lambda}{4 !}\left(\phi^{\dagger} \phi-\nu^{2}\right)^{2} & =\frac{\lambda}{4 !}(2 \nu+h)^{2} h^{2}
\end{aligned}
$$


Putting all the pieces together, (B.12) becomes:

$$
\begin{aligned}
\mathscr{L} & =\left(\partial_{\mu} h\right)^{2}+e^{2}(\nu+h)^{2} A_{\mu} A^{\mu}-\frac{1}{4} F_{\mu \nu} F^{\mu \nu}-\frac{\lambda}{4 !}(2 \nu+h)^{2} h^{2} \\
& =\left(\partial_{\mu} h\right)^{2}-\frac{\lambda}{6} \nu^{2} h^{2}-\frac{1}{4} F_{\mu \nu} F^{\mu \nu}+e^{2} \nu^{2} A_{\mu} A^{\mu}+\mathscr{L}_{i n t} .
\end{aligned}
$$

Again, we make the substitutions $h=1 / \sqrt{2} \phi_{A}, \nu \theta=1 / \sqrt{2} \phi_{B}$, and $m=\sqrt{\lambda / 12} \nu$. The result is,

$$
\mathscr{L}=\frac{1}{2}\left(\partial_{\mu} \phi_{A} \partial^{\mu} \phi_{A}\right)-m^{2} \phi_{A}+\frac{1}{4} F_{\mu \nu} F^{\mu \nu}+e^{2} m^{2} \frac{12}{\lambda} A_{\mu} A^{\mu}+\mathscr{L}_{i n t}
$$

By requiring local phase invariance and invoking continuous spontaneous symmetry breaking of (B.11), we have recovered a massive scalar particle $\phi_{A}$ and a massive gauge field $A^{\mu}$ with mass $m_{B}=\sqrt{12 e^{2} m^{2} / \lambda}$. In essence, by an astute choice of gauge, we have eliminated the massless Goldstone boson in (B.11) and recovered a massive vector field. Metaphorically, the gauge field "ate" up Goldstone boson and acquired a mass. This phenomenon is also explained through degrees of freedom (see for example [63]). A massless vector field, $A_{\mu}$ has 2 degrees of freedom, one for each polarization state. When it acquires mass, it has 3 degrees of freedom: 2 transverse states, and 1 longitudinal state. By fixing the gauge of the scalar field $\phi$ a degree of freedom is freed. It is precisely the local gauge invariance of the scalar field $\phi$ which afforded the vector field this third degree of freedom. This process is known as the "Higgs Mechanism." 


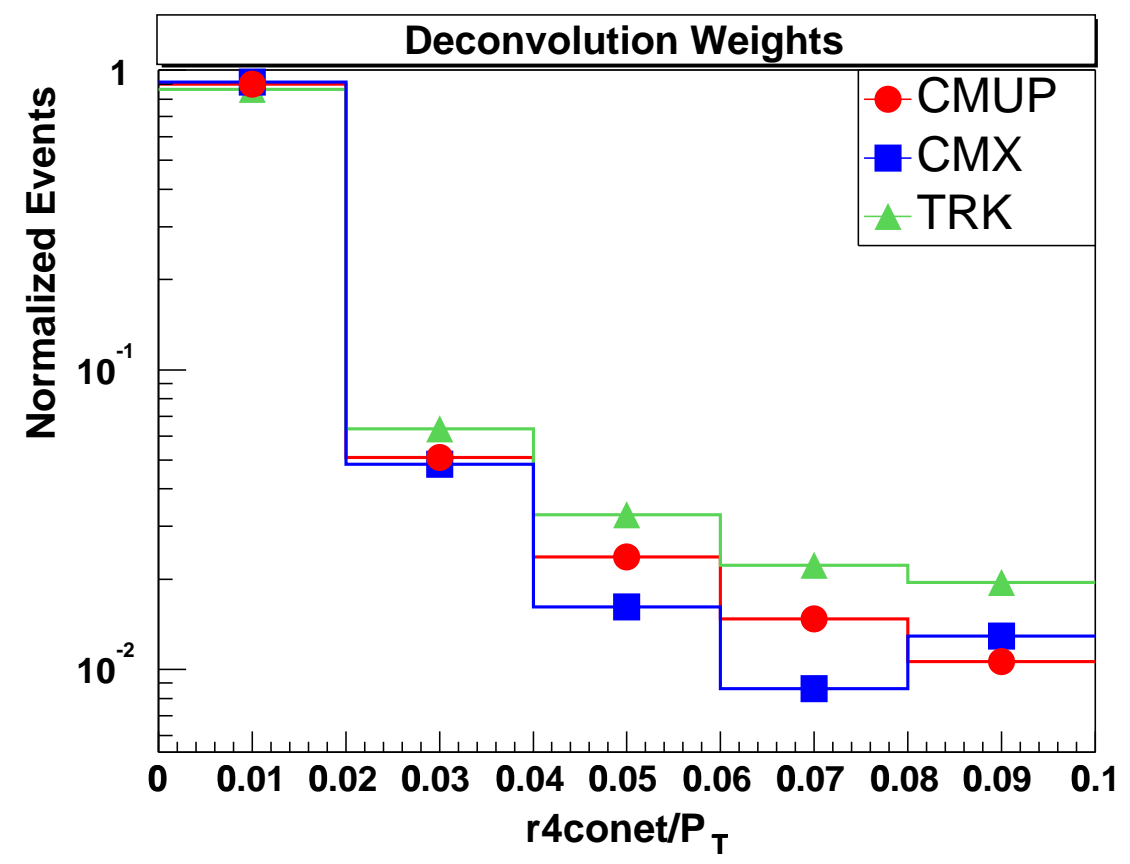

Figure B.2: The $\mathrm{M}(\mathrm{LQ})=240 \mathrm{GeV} / \mathrm{c}^{2}$ normalized isolation distributions for CMUP, CMX and TRK muons. 


\section{List of Tables}

1.1 The fundamental interactions and their properties. The typical interaction lifetime denotes the timescale for the decay of an unstable particle via that fundamental interaction. For example the mean lifetime for decay through a weak interaction is $10^{-10} \mathrm{~s} . \ldots \ldots \ldots 4$

1.2 The quark and lepton generations and their respective charges. $Q /|e|$ denotes the charge relative to the charge of the electron. The corresponding antiparticles have the opposite charge. . . . . . . . . . . . . . 5 5

2.1 A classification of leptoquarks in the Buchmüller-Rückl-Wyler leptoquark scheme [35] adopted from [94]. Shown is the leptoquark fermion number $F=3 B+L$, the electric charge $Q$ in units of $e$, the branching ratio $\beta$, and the lepton-quark couplings. The last column shows the possible squark assignments to the leptoquark states in the MSSM with broken R-parity. The classification assumes conjugation symmetry and the ability to globally replace the second-generation particles with first- or third-generation particles. 22

2.2 Theoretical scalar leptoquark cross-sections for $p \bar{p}$ collisions at $\sqrt{s}=1.96 \mathrm{TeV}$. 30

2.3 Published results of scalar leptoquark searches from the Run I Tevatron. $\mathcal{L}$ is the time-integrated luminosity and $M$ is the leptoquark mass below which scalar leptoquarks have been excluded at the $95 \%$ C.L. The $\tau \tau b b$ channel was analyzed by D $\varnothing$ using three sets of data collected using different triggers. . . 35

2.4 Preliminary results of scalar leptoquark searches at the Run I and Run II Tevatron. . . . . . . . . . . . . . . . . . 35

3.1 The components of the accelerator chain at Fermilab. The energy and destination of the beam depends on the operational mode of the accelerator. . . . 39

3.2 Acronyms of the CDF II calorimeter subtector types. . . . . . . . . . . . . 49

3.3 Granularity and indexing description of CDF II's eight tower types. . . . . . 49

3.4 Design specifications of the CMU, CMP, and CMX detectors . . . . . . . 53 
3.5 The trigger paths used in leptoquark search analysis. The number following the muon type denotes the $p_{T}$ trigger of the muon stub. The number follwing the "PT" denotes the $p_{T}$ of the XFT trigger. . . . . . . . . . . . 64

4.1 The level-3 trigger criterion used to filter the bhmu08 dataset. . . . . . . . 68

4.2 Efficiency summary table as from Ref. [80], [90], [95], and Appendix A. . . . 73

4.3 The muon ID selection cuts. TRK muons are not required to satisfy the track-stub match. . . . . . . . . . . . . . . 73

5.1 The total event-type efficiencies calculated using values from Table 4.2. $\quad$. . . 79

5.2 The ratios of efficiences between data and leptoquark Monte Carlo. The ratio is defined as data/MC. Scale factors calculated for the background Monte Carlo are not shown here. . . . . . . . . . . . . . . . . 83

5.3 Kinematic efficiency as a function of the leptoquark mass. . . . . . . . . . 83

5.4 List of events passing the selection cuts in $198 \mathrm{pb}^{-1}$ of data. The number of same-sign dimuon events found in the events passing the selection cuts is shown in the last column. . . . . . . . . . . . . . . . 85

5.5 Significant background processes and signal contamination. . . . . . . . 85

5.6 Surviving same-sign events after each kinematic cut. . . . . . . . . . 86

5.7 Parameters used in the calculation of the $\mathrm{Z}$ cross-section. . . . . . . . . . 87

5.8 The total acceptance percentages for each leptoquark mass listed with the statistical, systematic and combined relative errors. . . . . . . . . . 88

5.9 The measured upper limit on the expected number of background subtracted signal events in $198 \mathrm{pb}^{-1}$ for $L Q \overline{L Q} \rightarrow \mu q \mu q$. The right two columns show the theoretical expected number of scalar leptoquark events for different factorization/renormalization scales. . . . . . . . . . . . . . 92

5.10 Values of the upper limits at 95\% CL of the production cross-section of second generation leptoquarks decaying into $\mu q \mu q$ as a function of $M_{L Q}$. The last 2 columns on the right report the result of the theoretical calculations at NextTo-Leading order with CTEQ4M for different choices of the scale multiplied by a factor of $\beta=1 \ldots \ldots \ldots \ldots \ldots$. . . . . . . . . . . . . . . .

6.1 Mass distribution widths for $\mu$-jet1 and $\mu$-jet $2 \ldots \ldots . \ldots 96$

6.2 The acceptance efficiencies for the various criteria of jet assignment for the leptoquark mass cut. . . . . . . . . . . . . . . 98

6.3 Kinematic efficiency as a function of the leptoquark mass. . . . . . . . . 98 
6.4 The measured upper limit on the expected number of background subtracted signal events in $198 \mathrm{pb}^{-1}$ for $L Q \overline{L Q} \rightarrow \mu q \nu q$. The right two columns show the theoretically expected number of scalar leptoquark events for different factorization $/$ renormalization scales. . . . . . . . . . . . . . . 99

6.5 The background processes considered for the signal contamination. The expected background events column shows values obtained for a leptoquark mass $M_{L Q}=180 \mathrm{GeV} / \mathrm{c}^{2} \ldots \ldots \ldots \ldots \ldots$. . . . . . . . . 99

6.6 QCD background calculation regions. . . . . . . . . . . . 100

6.7 Events surviving the kinematic cuts in the regions defined in Table 6.6. The number of events surviving the mass cut in region $\mathrm{I}$ is for $M_{L Q}=180 \mathrm{GeV} / \mathrm{c}^{2} \cdot 100$

6.8 The final events calculated for expected backgrounds and observed events in the data for several scalar leptoquark masses. . . . . . . . . . . . . . . . 101

6.9 Parameters used in the calculation of the expected number of $W+\geq 2$ jet events . . . . . . . . . . . . . . . . . . . . 102

6.10 The total acceptance percentages for each leptoquark mass listed with the statistical, systematic and combined relative errors. . . . . . . . . . . 103

6.11 Values of the upper limits at 95\% CL of the production cross section of second generation leptoquarks decaying into $\mu q \nu q$ channel as a function of $M_{L Q}$. The last 2 columns on the right report the result of the theoretical calculations at Next-To-Leading order with CTEQ4M for different choices of the scale. The upper limit values have been normalized by a factor of $\beta=1 / 2 . \ldots 103$

7.1 Values of the 95\% CL lower limits on the second generation scalar leptoquark masses from CDF and D $\varnothing$ experiments. The mass limits in bold denote the results produced in this analysis. The combined results means that the limit was determined by combining results of two or more $\beta$ values. . . . . . . . . 113

A.1 Efficiencies obtained from the 1-leg method using $179 \mathrm{pb}^{-1}$ of data. The EM and HAD slide with the muon $P_{T}$ as described in Table $4.2 . \ldots 117$

B.1 The muon ID efficiencies deconvoluted from the muon isolation. . . . . . . 118 


\section{List of Figures}

1.1 Feynman diagram of nuclear $\beta$-decay. $\ldots \ldots \ldots \ldots \ldots$

1.2 The electron self-energy diagram in QED. . . . . . . . . . . . . . . 12

1.3 The fermion one-loop contribution to the SM Higgs self-energy diagram. . . 13

2.1 The strong, weak and electromagnetic couplings as a function of energy (from Kambara [76]). . . . . . . . . . . . . . . . . . 18

2.2 Feynman diagrams of the parton level process $g q \rightarrow L Q \ell$. The parameter $\lambda_{\ell q}$ represents the coupling between the lepton and quark. . . . . . . . . . . 23

2.3 Feynman diagrams of the parton level process $g g \rightarrow L Q \overline{L Q} . \quad \ldots \ldots$. . . . 24

2.4 Feynman diagrams of the parton level process $q \bar{q} \rightarrow L Q \overline{L Q}(5-6)$. . . . . . . 25

2.5 Example Feynman diagrams for virtual corrections involving self-energy (7) and vertex correction $(8) \ldots \ldots \ldots \ldots \ldots . \ldots \ldots$

2.6 Generic Feynman diagrams for gluon radiation. . . . . . . . . . . . 28

2.7 Generic Feynman diagrams for Compton scattering. . . . . . . . . . . . . 29

2.8 The theoretical scalar leptoquark cross section as a function of factorization/normalization scale at the Run II Tevatron. . . . . . . . . . . . . . 31

2.9 The theoretical scalar leptoquark cross section as a function of leptoquark mass at the Run I and Run II Tevatron. . . . . . . . . . . . . . . . 32

2.10 An example of a leptoquark induced FCNC decay process $B^{0} \rightarrow \mu \tau$. The leptoquark $L Q$ couples with more than one lepton-quark generation. . . . . . 33

2.11 Second generation scalar leptoquark decay for $\beta=1$ (left) and $\beta=1 / 2$

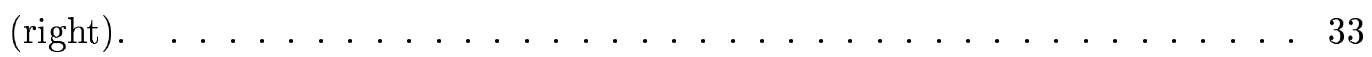

2.12 Limits on the first-generation scalar leptoquark mass as a function of the leptoquark coupling (from ZEUS Collaboration [104]). The limit for the Tevatron, which is independent of the leptoquark coupling, is from combined CDF and $\mathrm{D} \emptyset$ results from Run I. . . . . . . . . . . . . . . . . . . . 36 
3.1 An aerial diagram of the Run II accelerator chain. Although the Run II Tevatron was designed to run at $1 \mathrm{TeV}$, the Run II nominal energy is $960 \mathrm{GeV}$. It is divided into 6 sectors divided by straight sections labeled A0 through F0. The CDF II is located at B0. [23] . . . . . . . . . . . . . . . 40

3.2 A cross section schematic of the CDF II detector [42]. . . . . . . . . . . 44

3.3 A cross section of one quadrant in the $r-z$ plane of the CDF II tracking volume $[42] . \ldots \ldots \ldots \ldots \ldots \ldots$

3.4 A cross section of several COT drift chambers. The wires alternate between sense wires and potential wires. The arrow shows the relative direction of the nominal beam position [42]. . . . . . . . . . . . . . . . . 47

3.5 The east endplate showing the field planes of the COT cells. The numbers represent the radial distance from the beamline in centimeters[inches]. . . . . 48

3.6 A schematic of a CEM wedge [42] . . . . . . . . . . . . . . . 50

3.7 A schematic of a CES wedge [42]. . . . . . . . . . . . . . . 51

3.8 Location of the muon detector elements in the $\eta-\phi$ plane. The solid bands show the Run II coverage upgrades [42]. . . . . . . . . . . . . . . . . 54

3.9 A schematic of a CMU drift cell [42]. . . . . . . . . . . . . . . . 55

3.10 A schematic of an entire CMU module [42]. . . . . . . . . . . . . . . . 56

3.11 A schematic of an entire CMU module [42]. . . . . . . . . . . . . . 57

3.12 An illustration of the configuration of steel, CMP chambers and CSP scintillators looking in the $z$-direction. A muon track is drawn to indicate the interaction point. The two layers of scintillation counters are overlapped in the $z$-direction. . . . . . . . . . . . . . . . . 58

3.13 The number of interactions per bunch crossing as a function of luminosity for a bunch crossing of 132 ns and 396 ns (taken from [68]). . . . . . . . . . 61

3.14 A flowchart of the CDF II online data flow. The specifications for the trigger system were designed for a maximum bunch crossing period of 132 ns. . . . . 62

3.15 The Level-1 and Level-2 triggering systems. The TSI (Trigger Superviser Interface) provides an interface between the trigger system, data acquisition and clock.

4.1 Stub-track matching for CMU, CMX, and CMX muon types in the $M_{L Q}=$ $220 \mathrm{GeV} / \mathrm{c}^{2}$ Monte Carlo sample. . . . . . . . . . . . . . . 74

4.2 Electromagnetic and hadronic energy distributions for muons from leptoquark decay $\left(M_{L Q}=220 \mathrm{GeV} / \mathrm{c}^{2}\right) . \ldots \ldots \ldots \ldots \ldots \ldots$

$5.1 \quad P_{T}$ and $E_{T}$ distributions for leading (dark) and second leading (light) muons (left) and jets (right) for $M_{L Q}=220 \mathrm{GeV} / \mathrm{c}^{2}$. 
5.2 Sum of $\mathrm{E}_{T}$ (jets) versus sum of $\mathrm{P}_{T}$ (muons) for $M_{L Q}=220 \mathrm{GeV} / \mathrm{c}^{2}$ for Monte Carlo events. The plot is normalized to $2.5 \mathrm{fb}^{-1}$ and the size of the bins is 1 $\mathrm{GeV}$ by $1 \mathrm{GeV} / \mathrm{c}$.

5.3 The selection cut efficiencies as a function of leptoquark mass. The top curves show the relative efficiencies of the cuts in sequence, independent of both the preselection and the geometric detector acceptance. The bottom curve shows the final efficiency with the geometric acceptance included. The statistical errors are plotted. . . . . . . . . . . . . . . . . 8

5.4 The total signal acceptance efficiencies for the $\mu q \mu q$ channel. The plot shows the contributions to the total acceptance (black) and the acceptance without TRK muons (grey) from the different muon detectors. The statistical errors are plotted. . . . . . . . . . . . . . . . 8

5.5 Limit cross-section as a function of $M_{L Q}$ compared with the theoretical expectations calculated at NLO accuracy. At the intersection points between experimental and theoretical curves we find a lower limit on $M_{L Q}$ at 221

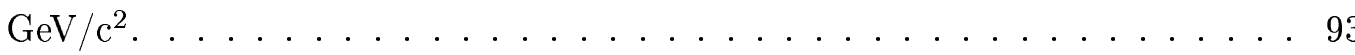

6.1 Reconstructed mass distributions for $M_{L Q}=180 \mathrm{GeV} / \mathrm{c}^{2}$. The red lines represent the cuts made based on the distribution widths and the generated leptoquark mass. . . . . . . . . . . . . . . . . . 97

6.2 The reconstructed mass distributions of the two leading jets and muon for Standard Model background, $M_{L Q}=180 \mathrm{GeV} / \mathrm{c}^{2}$ leptoquark signal, and observed data events. . . . . . . . . . . . . . . . . . . . . . . . 104

6.3 The reconstructed transverse mass distributions of the two leading jets and muon for Standard Model background, $M_{L Q}=180 \mathrm{GeV} / \mathrm{c}^{2}$ leptoquark signal, and observed data events. . . . . . . . . . . . . . . . . . . . 105

6.4 Cumulative efficiencies of the kinematic cuts for a range of leptoquark masses. The statistical errors are plotted. . . . . . . . . . . . . 106

6.5 The fractional muon isolation versus $E_{T}$ in $198 \mathrm{pb}^{-1}$ of data before any kinematic cuts. The QCD background dominates the region $\mathbb{E}_{T}<10 \mathrm{GeV}$; the $W+\geq 2$ jets and $\ell+$ jets dominates the region $10<E_{T} / \mathrm{GeV}<60$. . . 107

6.6 The fractional muon isolation versus $E_{T}$ in $198 \mathrm{pb}^{-1}$ of data before the $M_{T}>120 \mathrm{GeV} / \mathrm{c}^{2}$ cut. . . . . . . . . . . . . 108

6.7 Distributions of the invariant transverse mass of the muon and $\boldsymbol{E}_{T}$ for data (bullets) and Monte Carlo. . . . . . . . . . . . . . . . . . 109 
6.8 Limit cross section as a function of $M_{L Q}$ compared with the theoretical expectations calculated at NLO accuracy. At the intersection points between experimental and theoretical curves we find a lower limit on $M_{L Q}$ at 175 $\mathrm{GeV} / \mathrm{c}^{2} \ldots \ldots \ldots \ldots \ldots \ldots$

B.1 The muon ID efficiency versus the muon fractional isolation. Each event is required to have at lease one tight muon. . . . . . . . . . . . . . . . 119

B.2 The $\mathrm{M}(\mathrm{LQ})=240 \mathrm{GeV} / \mathrm{c}^{2}$ normalized isolation distributions for CMUP, CMX and TRK muons. . . . . . . . . . . . . . . . . . . . . . . 123 


\section{Bibliography}

[1] M. Krämar et al., Pair Production of Scalar Leptoquarks at the TeVatron, Phys. Rev. Lett. 79, 341 (1997).

[2] J.A. Grifols and A. Méndez, Phys. Rev. D 26, 324 (1982); I. Antoniadis, L. Baulieu, and F. Delduc, Z. Phys. C 23, 119 (1984); E. Eichten, I. Hinchliffe, K.D. Kane, and C. Quigg, Rev. Mod. Phys. C 56, 579 (1984); G. Altarelli and R. Rückl, Phys. Lett. B 144, 126 (1984); S. Dawson, E. Eichten, and C. Quigg, Phys. Rev. D 31, 1581 (1985); J. Blümlein, E. Boos, and A. Kryukov in [31].

[3] P. Arnold and C. Wendt, Phys. Rev. D 33, 1873 (1986); G.V. Borisov, Y.F. Pirogov, and K.R. Rudakov, Z. Phys. C 36, 217 (1987); J.L. Hewett, T.G. Rizzo, S. Pakvasa, H.E. Haber, and A. Pomarol, Argonne Accel. Phys., 539 (1993); J. Blümlein, E. Boos, and A. Kryukov in [31].

[4] H1 Collaboration, Adloff C. et al. Z. Physics C 74, 191 (1997).

[5] Nucl. Instr. and Meth. A425 86.

[6] L. Balka et al., Nucl. Instr. and Meth. A267, 272 (1988).

[7] F. Abe et al., Phys. Rev. D 50, 2296 (1994).

[8] Nucl. Instr. and Meth., A267, 272.

[9] Nucl. Instr. and Meth., A267, 301.

[10] Y. Seiya et al., Nucl. Instr and Meth. A480, 524 (2002).

[11] J. Conway. and K. Maeshima, Upper Limits on Poisson Processes Incorporating Uncertainties in Acceptance and Background, CDF Internal Note 4476, Version 2 (1998).

[12] M. Kuze, Y. Sirois, Search for Particles and Forces Beyond the Standard Model at HERA ep and Tevatron pp Colliders, DESY 02-165 (2002). 
[13] A. Mehta, HERA Upgrade and Prospects, X International Workshop on Deep Inelastic Scattering (DIS2002), Cracow, Poland (2002).

[14] J. Blümlein, E. Boos and A. Kryukov, Leptoquark pari production cross-sections at hadron colliders, [hep-ph/9811271].

[15] B. Dion, G. Simon Marleau and M. de Montigny, Eur. Phys. J. C2, 497 (1998); O.J.P. Éboli, R.Z. Runchal and T.L. Lungov, Phys. Rev. D 57, 1517 (1998).

[16] Atlas Collaboration, Atlas Technical Proposal, CERN/LHCC/99-15 (1999).

[17] Description of data samples for Top and Electroweak groups for Summer 2003. Description of data samples for Top and Electroweak groups for Summer 2003, CDF Note 6548 (2003).

[18] Searches for New Particles in High Mass Dimuon Channel in CDF Run II Data. Searches for New Particles in High Mass Dimuon Channel in CDF Run II Data, CDF Note 6344 (2003).

[19] Searches for New Particles in High Mass Dimuon Channel in CDF Run II Data. Searches for New Particles in High Mass Dimuon Channel in CDF Run II Data, CDF Note 6602 (2003).

[20] ROOT - An Object Oriented Data Analysis Framework, AIHENP conference, Lausane, (1996).

[21] New Results on Neutral and Charged Current Scattering at high $Q^{2}$ from H1 and ZEUS, Hamburg, Germany, July 28 - August 11997.

[22] L.F. Abbott and E. Farhi. Are the Weak Interactions Strong?, Phys. Lett. B 101, 69 (1981).

[23] Abid Patwa. Personal correspondence.

[24] D. Acosta, D. Konigsberg, J. Tsybychev, and Wang S.M. Update on the Search for First-Generation Leptoquarks in the Jets and MET Topology, CDF Internal Note 6593 (2003).

[25] A.D. Martin, R.G. Roberts, W.J. Stirling and R.S Thorne. Parton Distributions: a New Global Analysis. see also http://consult.cern.ch/writeup/pdflib/.

[26] I.J.R. Aitchison and A.J.G. Hey. Gauge Theories in Particle Physics. Institute of Physics Publishing, Ltd, Bristol, UK, (1989). 
[27] The CDF Trigger and Datasets Working Group Albrow, M. et al. CDF Run-II Trigger Table and Datasets Plan, CDF Internal Note 4718 (2001).

[28] B. Ashmankas. et al. Open-cell Chamber to Replace the CTC, CDF Internal Note 3648 (1996).

[29] W. Beenakker, H. Kuijf, and W.L. van Neervan. QCD corrections to heavy-quark

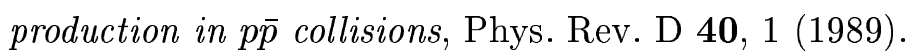

[30] A. Bhatti, M.D. Shapiro, and Lamoureux J. Combined Calorimetry Tower Elements, CDF Internal Note 3952 (1996).

[31] J. Blümlein, E. Boos, and A. Kryukov. Leptoquark Pair Production in Hadronic Interactions, [hep-ph/9610408] (1996).

[32] J. Botts, J.G. Morfin, J.F. Owens, J. Qiu, W. Tung, and H. Weerts. CTEQ Parton Distributions and Flavor Dependence of Sea Quarks, Phys. Lett. B 304, 159 (1993) [hep-ph/9303255].

[33] S.T. Buchmüller, R.D. Peccei, and T. Yanagida. The Structure of Weak Interactions for Composite Quarks and Leptons, Nuclear Physics B 244, 186 (1984).

[34] W. Buchmüller, S.T. Love, R.D. Peccei, and T. Yanagida. "Quasi Goldstone Fermions", Phys. Lett. B 115, 233 (1982).

[35] W. Buchmüller, R. Rückl, and D. Wyler. Leptoquarks in lepton-quark collisions, Phys. Lett. B 191, 442 (1987).

[36] CDF and DØ Collaborations. Combined Limits on First Generation Leptoquarks from the CDF and DØ Experiments, Fermilab Report Number FERMILAB-PUB-98-312-E (1997) [hep-ex/9810015].

[37] CDF Collaboration, F. Abe et al. Phys. Rev. Lett. 79, 4327 (1997).

[38] CDF Collaboration, F. Abe et al. Phys. Rev. Lett. 81, 4806 (1998).

[39] CDF Collaboration, F. Abe et al. Phys. Rev. Lett. 78, 2906 (1997).

[40] CDF Collaboration, F. Abe et al. The CDF Run II Silicon Tracking System, Nucl. Instrum. Meth. A447 (2000), pp. 1-8.

[41] CDF Collaboration, T. Affolder et al. Search for Second and Third Generation Leptoquarks Including Production via Technicolor Interactions in p $\bar{p}$ collisions at $\sqrt{s}=1.8$ TeV, Submitted to Phys. Rev. Lett., [hep-ex/0004003]. 
[42] The CDF Collaboration. The CDF II Detector Technical Design Report, Fermilab Report No. Fermilab-Pub-96/390-E (1996).

[43] R. Cousins. Why isn't every physicist a Bayesian?, Am. J. Phys. 65, 398 (1995).

[44] D. Amidei et al. Measurements of $\sigma \cdot W \rightarrow \mu \nu$ and $Z \rightarrow \mu \mu$ and $\frac{W \rightarrow \mu \nu}{Z \rightarrow \mu \mu}$ using $C D F$ Run II Data, CDF Note 6711 (2004).

[45] D. Bogert, W. Foster, W. Fowler, S. Holmes, G. Jackson, P. Martin, T. Pawlak. The Status of the Fermilab Main Injector Project, Prepared for 5th European Particle Accelerator Conference (EPAC 96), Sitges, Spain, 10-14 Jun (1996).

[46] DØ Collaboration, B. Abbott et al. Phys. Rev. D 64, 092004 (2001).

[47] DØ Collaboration, B. Abbott et al. Phys. Rev. Lett. 79, 4321 (1998).

[48] DØ Collaboration, B. Abbott et al. Phys. Rev. Lett., 84, 2088 (2000).

[49] DØ Collaboration, B. Abbott et al. Phys. Rev. Lett. 83, 2896 (1999).

[50] DØ Collaboration, B. Abbott et al. Phys. Rev. Lett. 81, 38 (1998).

[51] DØ Collaboration, V.M. Abazov et al. Phys. Rev. Lett. 88, 191801 (2002).

[52] Manuel Drees. An Introduction to Supersymmetry, [hep-ph/9611409v1] (1996).

[53] O.J.P. Éboli and T.L. Lungov. "Single production of leptoquarks at the Tevatron", Phys. Rev. D 61, 75015 (2000).

[54] F. Abe et al., CDF Collaboration. Search for First Generation Leptoquark Pair Production in p $\bar{p}$ Collisions at $\sqrt{s}=1.8$ TeV, [hep-ex/9707033v1] (1997).

[55] E. Farhi and L. Susskind. Technicolour, Phys. Rep. 74 No. 3, 277 (1981).

[56] Averaging of the inelastic cross sections measured by the CDF and the E811 experiments, Fermilab Report No. Fermilab-FN-0741 (2003).

[57] Y. Fukuda and et al. Evidence for Oscillation of Atmospheric Neutrinos, Phys. Rev. Lett. 81, 1562 (1999).

[58] G. Ascoli et al. CDF Central Muon Detector, Nucl. Inst. and Meth. A269, 33 (1988).

[59] G. Brandenburg et al. . The CDF Run 1 Muon System Upgrade, CDF Internal Note 6362 (2002). 
[60] H. Georgi and S.L. Glashow. Unified theory of elementary-particle forces, Physics Today, 30-39 (1980).

[61] H. Georgi and S.L. Glashow. Unity of All Elementary-Particle Forces, Phys. Rev. Lett. 32, 438 (1974).

[62] G.J. Feldman and R.D. Cousins. Unified approach to the classical statistical analysis of small signals, Phys. Rev. D 57, 3873 (1998).

[63] David Griffiths. Introduction to Elementary Particles. John Wiley and Sons, Inc., New York, NY, (1987).

[64] S.L. Glashow H. Georgi. Gauge Theories Without Anomalies, Phys. Rev. D 6, 429 (1972).

[65] H1 BSM Working Group. New Results on NC and CC-like Events at Very High $Q^{2}$ in DIS Collisions at HERA, July 1997.

[66] Brian Hatfield. Quantum Field Theory of Point Particles and Strings. Addison-Wesley, Reading, MA, (1992).

[67] J.L. Hewett and S. Pakvasa. Sclalar-leptoquark production at hadron colliders, Phys. Rev. D 37, 3165 (1988).

[68] R. Hughes, E. Thomson, and B. Winer. Performance Study of the eXtremely Fast Tracker at High Luminosity, CDF Internal Note 5986 (2002).

[69] J. Abbott et al., DØ Collaboration. Search for Scalar Leptoquark Pairs Decaying to

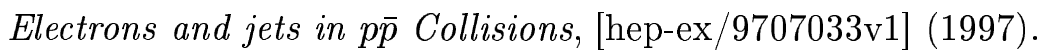

[70] J. Campbell, R.K. Ellis and D. Rainwater. Next-to-leading order QCD predictions for $W+2$ jet and $Z+2$ jet production at the CERN LHC, Fermilab Report No. FERMILAB-Pub-03/234-T, [hep-ph/0308195] (2003); see also http: ://mcfm.fnal. gov/.

[71] J. Elias. et al. Luminosity monitor based on Cherenkov counters for p $\bar{p}$ colliders, Submitted to Nucl. Inst. Meth.

[72] J. Konsigsberg J. et al. Luminosity Information in Run II, CDF Internal Note 4831 (1999).

[73] José Wudka. Composite Leptoquarks, Phys. Lett. B 167, 337 (1986).

[74] K. Byrum et al. Nucl. Inst. and Meth. A364, 144 (1995). 
[75] Michio Kaku. Quantum Field Theory. Oxford University Press, New York, NY, (1993).

[76] Hisanori Kambara. PhD thesis. Ph.D. Thesis: Search for Second Generation Leptoquarks in $\sqrt{s}=1.8 \mathrm{TeV} p \bar{p}$ at $C D F$, University of Genève (1998).

[77] B. Kilminster, K. McFarland, and K. Tollefson. Design and Specifications for the CDF run II Conumer-Server/Logger, CDF Internal Note 4794 (1998).

[78] M. Krämar. Leptoquark Production at the Tevatron, Talk presented at "Beyond the Desert 97", Accelerator and Non-Accelerator Approaches, Castl Ringberg, Tegernsee, Germany, [hep-ph/9707422] (1997).

[79] La Biodola. Event Builder and Level 3 at the CDF Experiment, 9th Pisa Meeting on Advanced Detectors, Isola d'Elba, Italy, May 2003. FERMILAB-CONF-03/172-E.

[80] V. Martin and L. Cerrito. Muon Cuts and Efficiencies for 4.11.1 Analyses, CDF Note 6825 (2004).

[81] M.L. Mangano, M. Moretti, F. Piccinini, R. Pittau, A. Polosa. JHEP 0307:001, (2003) [hep-ph/0206293]; see also http://mlm.home.cern.ch/mlm/alpgen/.

[82] Lorenzo Moneta. Combined Results of Searches for Second Generation Leptoquarks, CDF Internal Note 5790 (2001).

[83] Lorenzo Moneta. Searches for Second Generation Leptoquarks in $\mu \nu q q$ channel, CDF Internal Note 5572 (2001).

[84] L. Nodulman. Central Electron Issues for Summer 03, CDF Internal Note 5572, version $0.2(2003)$.

[85] Donald H. Perkins. Introduction to High Energy Physics. Cambridge University Press, Cambridge, UK, 4th edition, (2000).

[86] S. Rolli. Search for First Generation Leptoquarks Pair Production in the eejj Channel. March 2003. Search for First Generation Leptoquarks Pair Production in the eejj Channel, CDF Note 6338 (2003).

[87] S. Rolli, D. Ryan, and H. Sun. Search for 2nd Generation Scalar Leptoquarks, CDF Note 6733 (2003).

[88] S. Eidelman et al. Phys. Lett. B 592 (2004).

[89] W. Sakamoto. $W / Z$ Cross Section Predictions for $\sqrt{s}=1.96$ TeV, CDF Note 6341 (2003). 
[90] W.K. Sakumoto and A. Hocker. Event $\mid$ Zvtx $\mid<60 \mathrm{~cm}$ Cut Efficiency for Run II, CDF Note 6331 (2003).

[91] P.C. Salam and A. Salam. Lepton Number as a Fourth Color, Phys. Rev. D 10, 275 (1974).

[92] B. Schrempp and F. Schrempp. Light Leptoquarks, Phys. Lett. B 153, 101 (1985).

[93] B. Schrempp and F. Schrempp. A Confining $S U(2)_{L} \times S U(2)_{R}$ Gauge Model of the Weak Interactions, Nuclear Physics B 231, 109 (1984).

[94] Federica Strumia. PhD thesis. Searches for First Generation Leptoquarks in the eeqq and enuqq channels, University of Geneva (2002).

[95] T. Pratt et al. Search for Resonances in High Mass Dielectron, CDF Note 6073 (2003).

[96] T. Sjöstrand, P. Edén, C. Friberg, L. Lönnblad, G. Miu, S. Mrenna and E. Norrbin. see also http://www.thep.lu.se/ torbjorn/Pythia.html.

[97] A. Taffard. Run II Cosmic Ray Tagger, CDF Internal Note 6100 (2003).

[98] CDF Data Quality Monitoring Team. http://www-cdf.fnal.gov/internal/dqm/ goodrun/good.html.

[99] The CDF Run II Jet Corrections Group, J.F. Arguin et al. Generic Jet Energy Corrections for Run II data used for the Winter Confrerences, CDF Internal Note 6280, version 0.2 (2003).

[100] Thomson, E. and et al. Online Track Processor for the CDF Upgrade, IEEE Transactions on Nuclear Science 49, No. 3, Fermilab Report No. FERMILAB-CONF-02/144-E (2002).

[101] G. Valencia and S. Willenbrock. Quark-lepton unification and rare meson decays, Phys. Rev. D 50, 1 (1994).

[102] S. Weinberg. Implications of dynamical symmetry breaking, Phys. Rev. D, 13, 974 (1976).

[103] H. Wenzel and K. Maeshima. Search for Pati-Salam Leptoquarks via the decays $B_{S} \rightarrow$ $e^{ \pm} \mu^{\mp}$ and $B_{d} \rightarrow e^{ \pm} \mu^{\mp}, \mathrm{CDF}$ Note 4224 (1998).

[104] ZEUS Collaboration. Search for Leptoquarks in ep Collisions at HERA, Submitted to the XXXIst International Conference on High Energy Physics, Amsterdam, The Netherlands (2002). 
[105] ZEUS Collaboration, J. Breitweg et al. Z. Phys. C 74, 204 (1997). 